\title{
Different inter-domain linker regions regulate the binding of UHRF1 and NP95 to histone H3
}

\author{
Dissertation \\ for the award of the degree \\ “Doctor rerum naturalium" (Dr. rer. nat.) \\ Division of Mathematics and Natural Sciences \\ of the Georg-August-Universität Göttingen
}

submitted by

Maria Tauber

born in

Zwickau, Germany

Göttingen, 2016 


\section{Thesis Committee Members}

Prof. Dr. Wolfgang Fischle ( $1^{\text {st }}$ reviewer), Research Group Chromatin Biochemistry Max Planck Institute for Biophysical Chemistry, Göttingen King Abdullah University of Science and Technology, Thuwal, KSA

Prof. Dr. Michael Kessel ( $2^{\text {nd }}$ reviewer), Research Group Developmental Biology

Max Planck Institute for Biophysical Chemistry, Göttingen

Prof. Dr. Heinz Neumann, Research Group Applied Synthetic Biology

Göttinger Zentrum für Molekulare Biowissenschaften, Göttingen

Georg-August-University, Göttingen

Dr. Manfred Konrad, Research Group Enzyme Biochemistry

Max Planck Institute for Biophysical Chemistry, Göttingen

PD Dr. Halyna Shcherbata, Research Group Gene Expression and Signaling

Max Planck Institute for Biophysical Chemistry, Göttingen

Prof. Dr. Heidi Hahn, Section of Developmental Genetics

University Medical Center Göttingen

Date of oral examination: 17.06.2016 
Affidavit

I affirm that the presented thesis, "Different inter-domain linker regions regulate the binding of UHRF1 and NP95 to histone H3", has been written independently and with no other sources and aids than quoted.

Göttingen, March 31, 2016

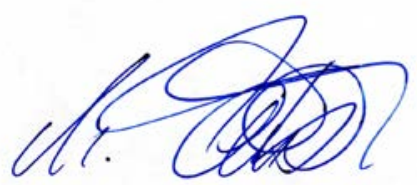

Maria Tauber 



\section{Acknowledgements}

First and foremost, I want to thank my supervisor Dr. Wolfgang Fischle for giving me the opportunity to work on this interesting project. I am grateful for his constant support, our fruitful discussions and his constructive criticism.

I want to thank my thesis committee members Prof. Dr. Michael Kessel and Prof. Dr. Heinz Neumann for their interest in my project and the fruitful discussions and interesting suggestions during my committee meetings and beyond.

I want to thank the GGNB for the constant support, informative lectures and highly supportive method courses.

I am grateful to Prof. Dr. Henning Urlaub and his group for their mass spectrometry work. In particular, I want to thank Aleksandar Chernev for his excellent work in the intramolecular crosslinking project and for his helpful advices and explanations.

I want to thank Prof. Nullin Divecha and his group for providing the expression constructs for the PIP4Ks.

I also want to thank Prof. Dr. Hiroaki Suga and his group for their efforts in our small molecule screening. Especially, I am thankful to Shiori Umemoto for her enthusiasm and her careful work.

I am thankful to Prof. Cheryl Arrowsmith and her group for their outstanding work in the structural analysis of UHRF1 and NP95. In particular, I am grateful to Alexander Lemak and Scott Houliston for their efforts and fruitful discussions.

I want to thank Prof. Tim Friede, PD Dr. Klaus Jung and Alexander Wolff for their counsel and their efforts in the mRNA expression data analysis.

I want to thank all lab members of the Fischle group for the pleasant time throughout my thesis. In particular, I am thankful to Dr. Kathy Gelato, Dr. Kyoko Hamada and Dr. Sarah Kreuz for the constant help and advice. I am especially grateful to Kyoko, Sarah, Nadin and Henriette for their friendship and the valuable hours spend outside of the lab.

I am thankful to my family. In particular I want to thank my parents Ute and Tomas Tauber and my brother Friedrich for constantly supporting and encouraging me to go all the way.

Lastly, I owe my gratitude to Jan-Philipp Spies for his constant support. 


\section{Table of contents}

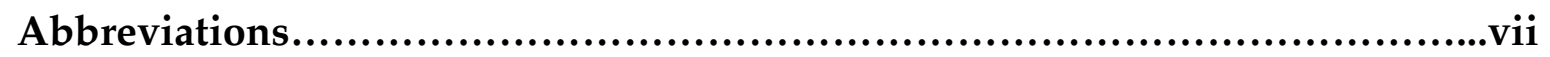

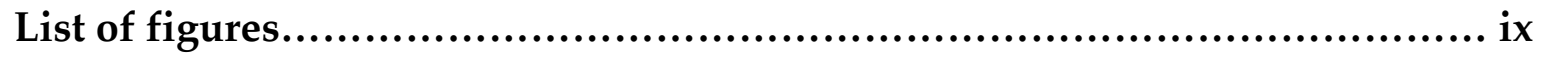

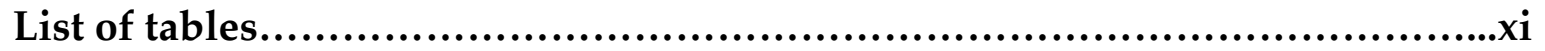

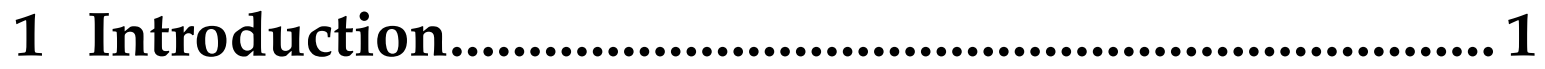

1.1 Epigenetics and chromatin ……..................................................................... 1

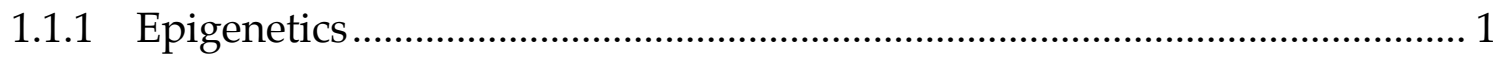

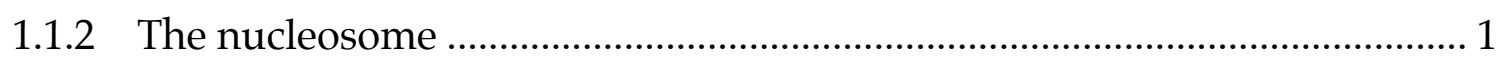

1.1.3 Higher order chromatin structures ................................................................ 3

1.2 Epigenetic modifications ..................................................................................... 4

1.2.1 Histone posttranslational modification …………………………………...... 4

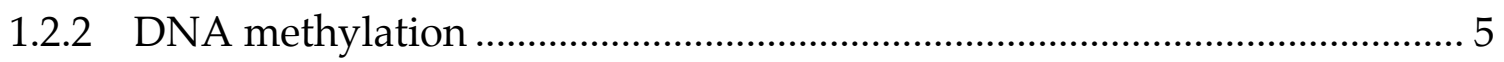

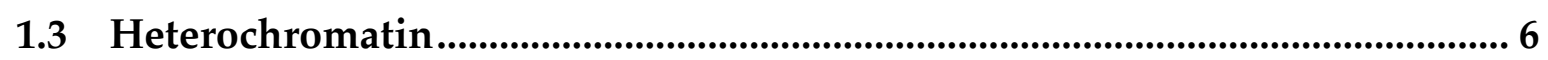

1.3.1 Chromatin domains - Euchromatin and Heterochromatin ........................... 6

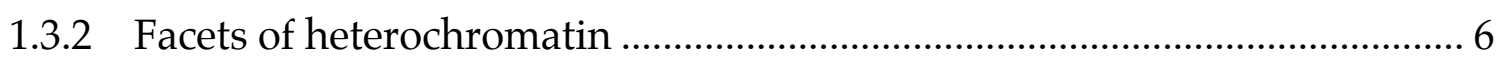

1.3.3 Maintenance of constitutive heterochromatin............................................... 7

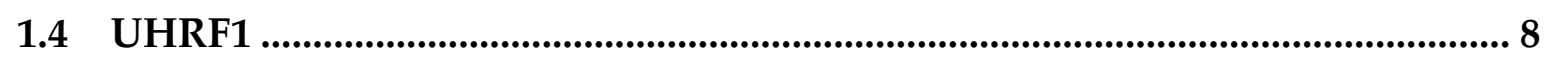

1.4.1 Function of UHRF1 .............................................................................. 8

1.4.2 Sequence conservation of UHRF1 proteins .................................................. 9

1.4.3 UHRF1 - Domain structure and function...................................................... 9

1.4.4 TTD-PHD interplay in recognizing the H3-tail........................................... 12

1.4.5 Control of TTD/H3K9me3 interaction by a C-terminal polybasic region 13

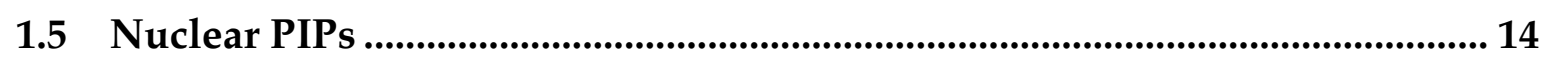

$1.5 .1 \quad \mathrm{PI}(5) \mathrm{P}$............................................................................................. 14

1.5.2 Phosphoinositol dependent regulation of chromatin factors...................... 14

1.5.3 Regulation of UHRF1 TTD/ PBR interaction by PI(5)P ............................. 16

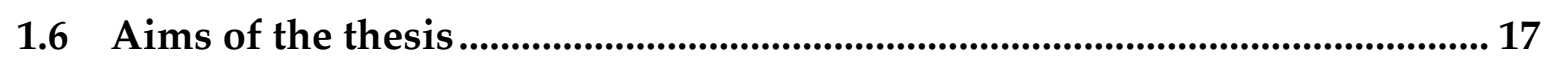

2 Material and Methods .............................................. 19 


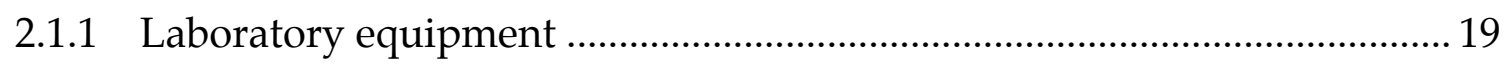

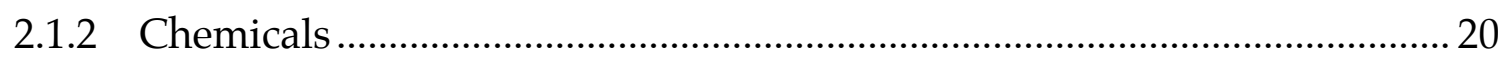

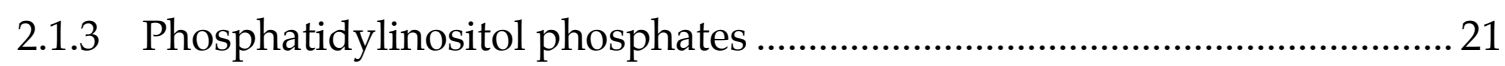

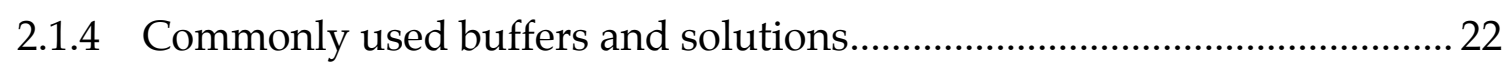

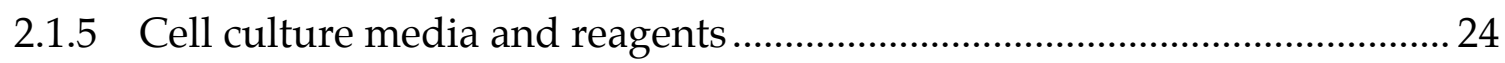

2.1.6 Chromatographic materials and consumables ..........................................25

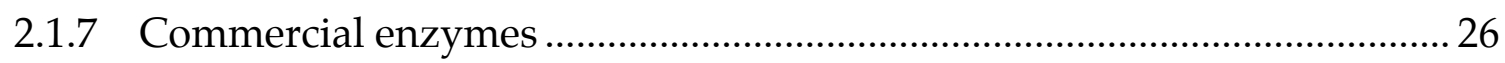

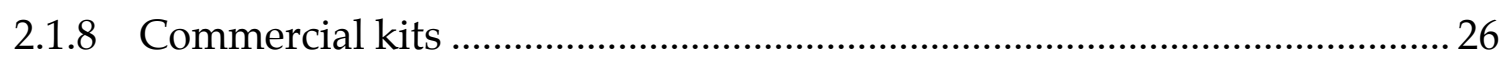

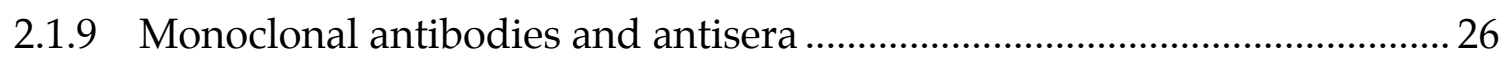

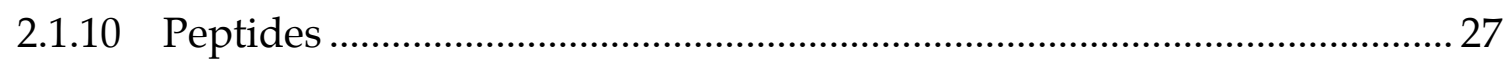

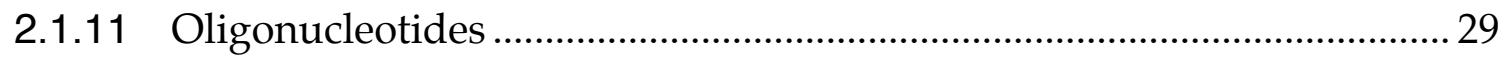

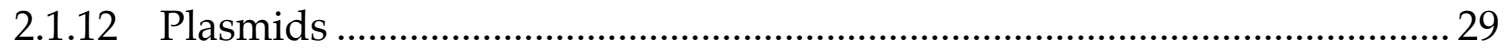

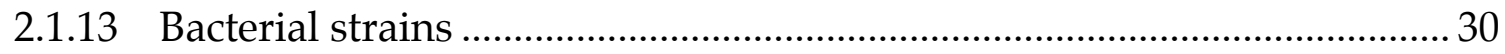

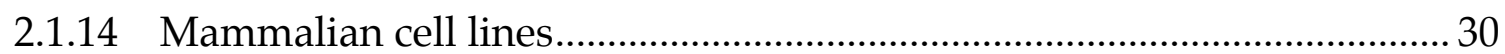

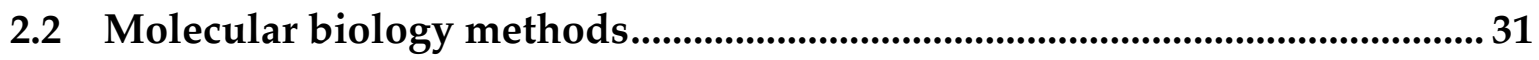

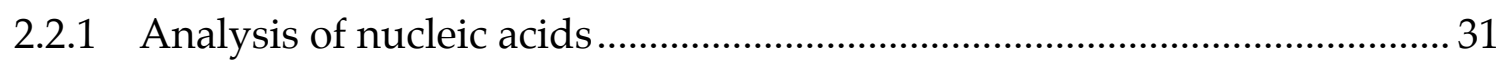

2.2.1.1 Spectrophotometric determination of the concentration of nucleic acids ......31

2.2.1.2 Agarose gel electrophoresis...................................................................................31

2.2.2 Cloning and mutagenesis procedures ....................................................... 31

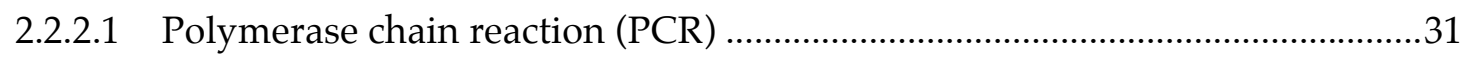

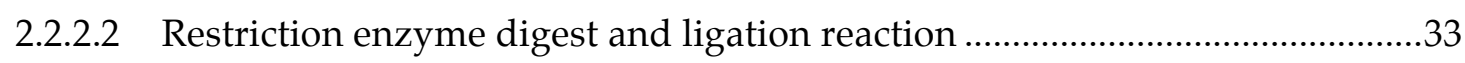

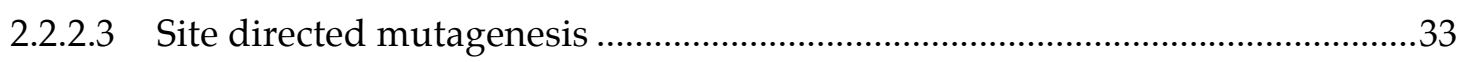

2.2.2.4 Preparation and transformation of chemically competent bacteria ..................34

2.2.2.5 Propagation and preparation of plasmid DNA ....................................................

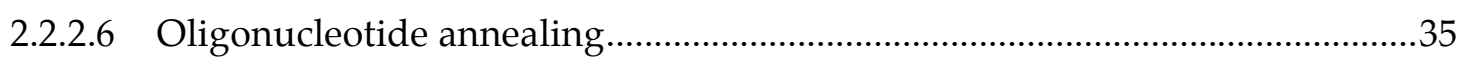

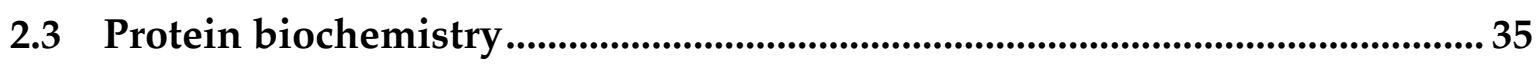

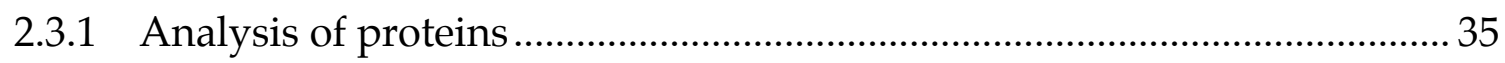

2.3.1.1 Spectrophotometric determination of protein concentration............................35

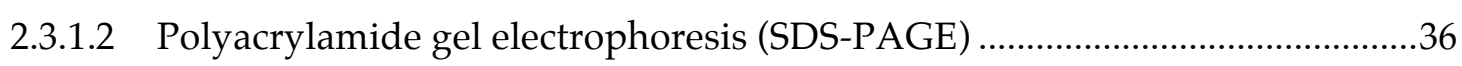

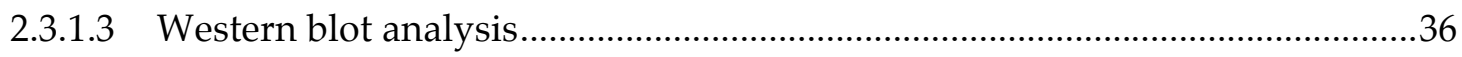

2.3.1.4 Coomassie staining of polyacrylamide gels .........................................................37

2.3.2 Expression and purification of proteins .................................................... 37

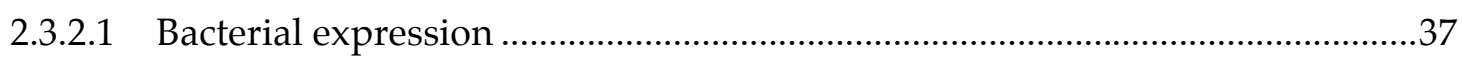

2.3.2.2 Purification by metal ion affinity chromatography ........................................38

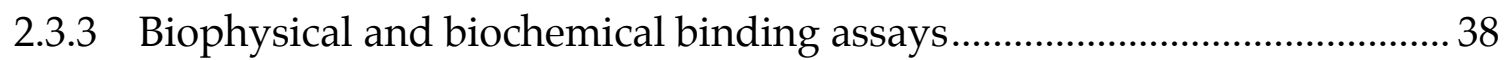




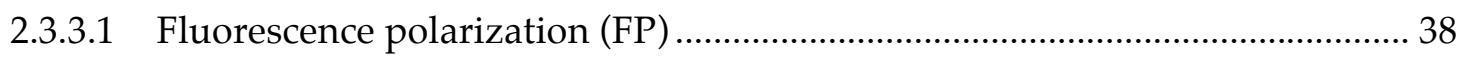

2.3.3.2 Peptide pull down assay with recombinant protein (PPD) ............................. 39

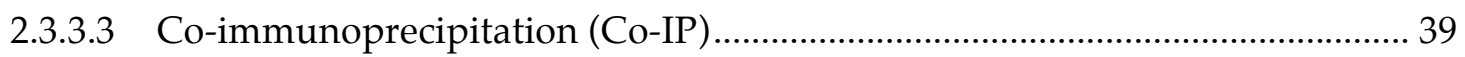

2.3.3.4 Cyclic peptide screening ......................................................................................... 41

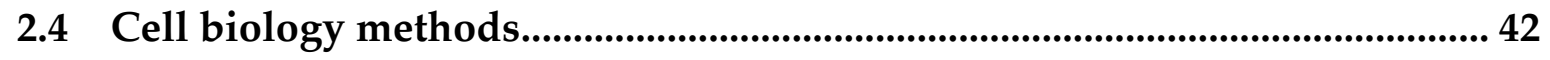

2.4.1 Cell culture, transfection and synchronization ........................................... 42

2.4.1.1 Passaging of mammalian cell lines .................................................................. 42

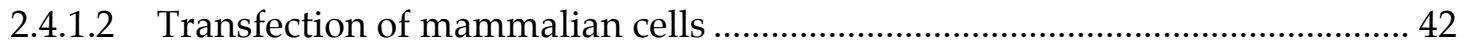

2.4.1.3 Synchronisation of mammalian cells............................................................. 43

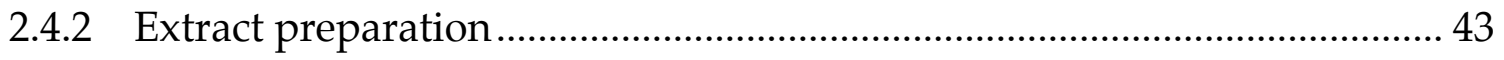

2.4.2.1 Cytosolic and nuclear extract preparation (rapid method).............................. 43

2.4.2.2 Nuclear extract preparation with MNase ................................................................ 44

2.4.3 Staining procedures of mammalian cells .................................................... 44

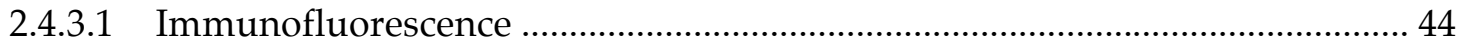

2.4.3.2 Nuclear counterstaining with propidium iodide (PI) staining....................... 45

2.4.3.3 Nuclear counterstaining with Hoechst 33342 staining ...................................... 45

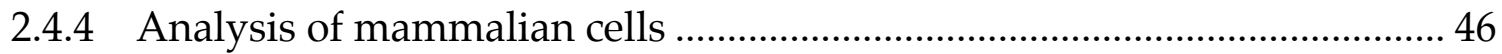

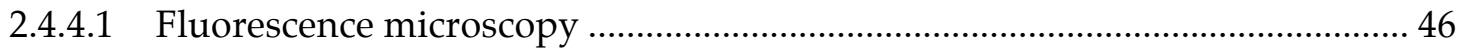

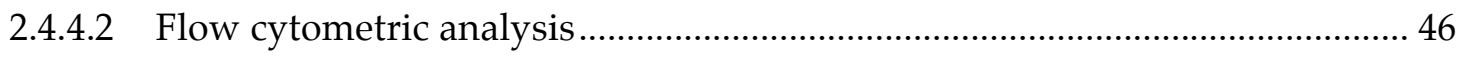

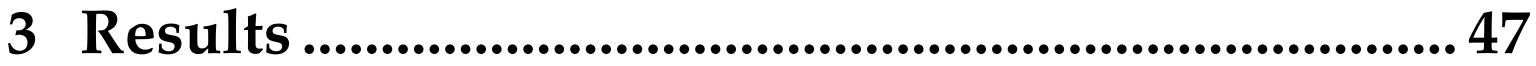

3.1 UHRF1 localization in cells is dependent on linker regions .......................47

3.1.1 Heterogeneous nuclear localization of UHRF1 ......................................... 47

3.1.2 The TTD domain is responsible for the localization of UHRF1 to

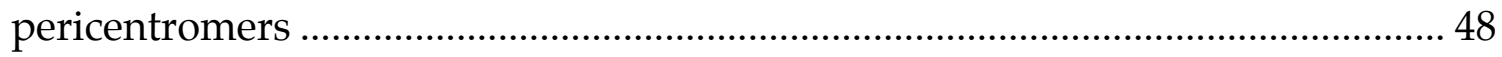

3.1.3 The PBR directs the TTD-dependent nuclear localization of UHRF1....... 51

3.1.4 Nuclear PI(5)P directs TTD-dependent localization of UHRF1 ................. 56

3.1.5 Novel cyclic peptides regulate the TTD / PBR interaction ........................ 57

3.2 NP95 binding behavior is dependent on its linker regions.......................... 60

3.2.1 The primary structures of TTD-PHD linker and PBR domains are highly

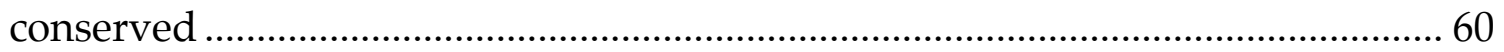

3.2.2 The PBR region does not regulate NP95 binding to H3K9me3 ................. 62

3.2.2.1 Comparison of UHRF1 and NP95 binding to the H3 tail using FP ................ 62

3.2.2.2 Full-length wild type UHRF1 and NP95 I show similar H3 tail binding ...... 64

3.2.2.3 Mutation of the PBR in NP95 affects binding to the H3 tail ............................. 66 
3.2.2.4 The PBR region does not affect TTD binding to H3K9me3 in NP95

3.2.3 NP95 binding to H3 tail is regulated by different TTD-PHD linker regions .70

3.2.3.1 The TTD-PHD region of UHRF1 and NP95 II shows similar H3 tail binding . .70

3.2.3.2 The TTD domain is blocked in NP95 I but not NP95 II....................................72

3.2.3.3 The NP95 I TTD is blocked by the TTD-PHD linker insertion ..........................74

3.2.3.4 The NP95 I TTD-PHD linker insertion changes UHRF1 binding behavior ...76 3.2.4 An R-K-S motif in the TTD-PHD linker influences binding of NP95 II to

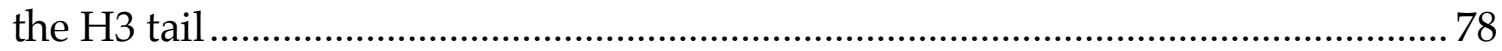

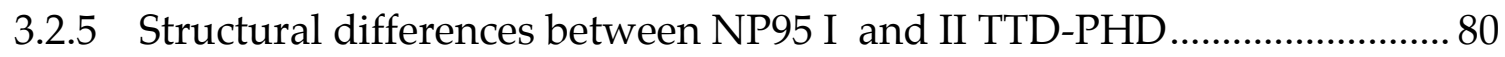

3.2.5.1 Similar structural features of TTD-PHD region of UHRF1 and NP95 II but not NP95 I. .80

3.2.5.2 TTD-PHD linker causes structural differences in the TTD of NP95 I and NP95 II

3.2.6 Different intramolecular interactions in UHRF1 and the NP95 isoforms 83

3.2.6.1 Common intramolecular crosslinks of NP95 and UHRF1 ..............................83

3.2.6.2 Different crosslink positions in the NP95 isoforms ..........................................8

3.2.6.3 DNA induced changes in intramolecular crosslinking of UHRF1 / NP95 .....85

3.2.6.1 Impact of DNA on the H3 tail binding of UHRF1 / NP95 ...............................86

3.3 Expression and cellular localization of NP95................................................8 88

3.3.1 Comparison of the nuclear localization of UHRF1 and NP95 ...................8 88

3.3.2 mRNA expression analysis of NP95 I and NP95 II.................................. 91

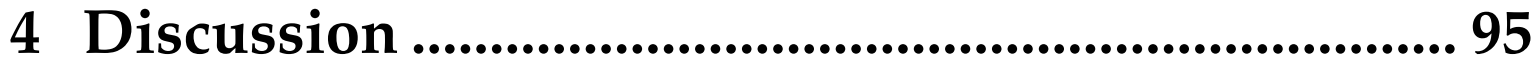

4.1 Model for linker-dependent allosteric transition in UHRF1 ......................... 95

4.2 Inter-domain linker dependent regulation of NP95 binding to H3K9me3.96

4.2.1 The PBR dependent blocking of the TTD in UHRF1 is not conserved in the two NP95 isoforms .96

4.2.2 The binding states of UHRF1 are resembled by the two splicing variants of NP95 in vitro .97

4.3 Inter-domain linker regions regulate the multivalent read out of chromatin marks in UHRF1 and NP95 .98

4.3.1 TTD/linker interaction is a prerequisite for a cooperative binding event in UHRF1 but not in NP95. .98 
4.3.2 Inter-domain linker regions improve binding specificity for multivalent targets 100

4.4 Linker influence the nuclear localization of UHRF1/NP95 ......................... 100

4.4.1 Similar cellular roles of UHRF1 and NP95 I ............................................ 100

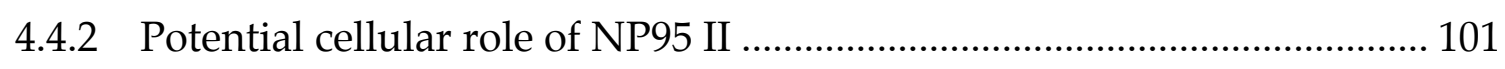

4.5 Regulation of the TTD interaction by different inter-domain linker regions

4.5.1 Characteristic features of linker regulations.............................................. 103

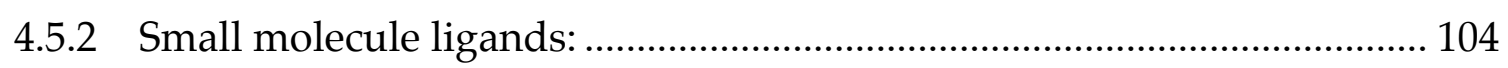

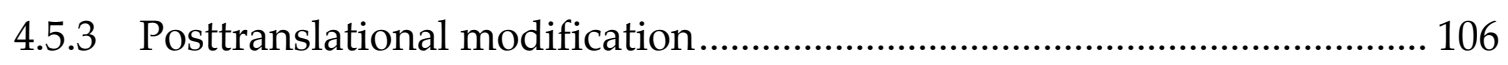

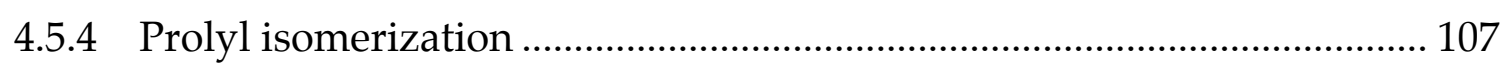

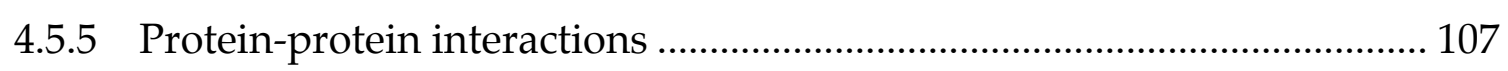

4.6 Alternative splicing of linker regions ............................................................. 109

4.7 Using small molecules to target UHRF1 in cancer.......................................... 110

5 Conclusion................................................................... 111

6 References........................................................................ 112

7 Addendum...................................................................... 127

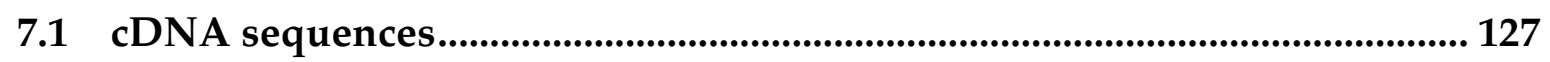

7.2 Primer list........................................................................................................ 130

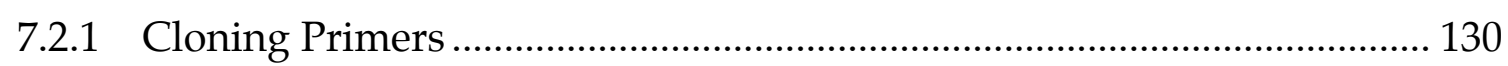

7.2.2 Sequencing primer............................................................................ 130

7.2.3 Mutagenesis primers................................................................................ 131

7.3 Mass spectrometry results ................................................................................ 132

8 Curriculum vitae ......................................................... 137

9 Publications related to thesis work ........................ 138 


\section{Abbreviations}

\begin{tabular}{|c|c|c|c|}
\hline${ }^{\circ} \mathrm{C}$ & degree Celsius & OD & optical density \\
\hline $\mathrm{A}$ & absorbance or ampere & PAGE & $\begin{array}{l}\text { polyacrylamide gel } \\
\text { electrophoresis }\end{array}$ \\
\hline aа & amino acid(s) & PBR & noly basic reoion \\
\hline bp & base pairs & & pory wasic Iegivit \\
\hline btn & biotin tag & PBS & phosphate buffered saline \\
\hline c & concentration or centi & PCR & polymerase chain reaction \\
\hline $\mathrm{Da}$ & Dalton $(\mathrm{g} / \mathrm{mol})$ & PHD & plant homeodomain \\
\hline DNA & deoxyribonucleic acid & RNase & ribonuclease \\
\hline e. coli & Escherichia coli & PPD & peptide pull down \\
\hline 6-FAM & 6-carboxyfluorescein & rpm & rotation per minute \\
\hline FL & full length & $\mathrm{RT}$ & room temperature \\
\hline FP & fluorescence polarization & sec & second \\
\hline $\mathrm{g}$ & gram & TTD & tandem tudor domain \\
\hline $\mathrm{h}$ & hour(s) & $\mathrm{U}$ & unit(s) \\
\hline His-tag & Histidine tag & UV & ultraviolet \\
\hline HRP & horseradish peroxidase & $\mathrm{V}$ & volt \\
\hline Hor & 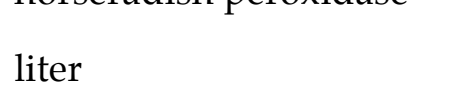 & $\mathrm{v} / \mathrm{v}$ & volume per volume \\
\hline LB & lysogeny broth & WT & wild type \\
\hline $\mathrm{m}$ & milli or meter & $\mathrm{w} / \mathrm{v}$ & weight per volume \\
\hline M & molar & $\mathrm{xg}$ & times gravity force \\
\hline Max & maximum & $\alpha$ & anti-/antibody \\
\hline Min & minimum & $\lambda$ & wavelength \\
\hline $\min$ & minute(s) & $\mu$ & micro \\
\hline MNase & Micrococcal nuclease & & \\
\hline $\mathrm{mt}$ & mutant & & \\
\hline MWCO & molecular weight cut off & & \\
\hline $\mathrm{n}$ & nano & & \\
\hline $\mathrm{o} / \mathrm{n}$ & over night & & \\
\hline
\end{tabular}




\section{List of Figures}

Figure 1-1 The nucleosome core particle structure and the histone-fold heterodimers ........2

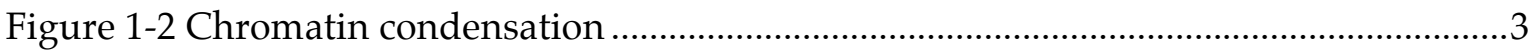

Figure 1-3 UHRF1 is a multi-domain factor with several conserved protein motifs and linker regions . .10

Figure 1-4 Regulation of chromatin interaction of several known factors by PIs..................15

Figure 2-1 Flow scheme of cyclic peptide screening procedure..............................................41

Figure 3-1 UHRF1 nuclear localization in different cell lines ................................................48

Figure 3-2 Nuclear localization of UHRF1 wild type and mutant in NIH3T3 cells ..............50

Figure 3-3 Nuclear localization of the UHRF1 PBR mutant in NIH3T3 cells .........................52

Figure 3-4 PBR-RING changes the nuclear localization of wild type UHRF1.......................54

Figure 3-5 Influence of PI(5)P on the nuclear localization of UHRF1 ......................................55

Figure 3-6 Regulation of the TTD / PBR interaction by cyclic peptides ..................................58

Figure 3-7 Sequence conservation of TTD-PHD linker and PBR ...........................................61

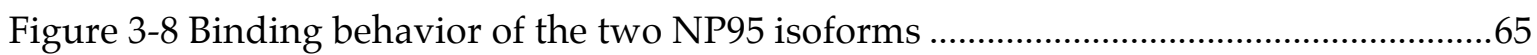

Figure 3-9 PBR mutations of NP95 I and II affect the binding to the H3 tail......................67

Figure 3-10 Weak interaction of the TTD-PHD and PBR-RING region of NP95 ...................69

Figure 3-11 Binding behavior of the TTD-PHD fragments of the two NP95 isoforms ........71

Figure 3-12 Differential H3 tail binding by PHD mutants of the two NP95 TTD-PHD

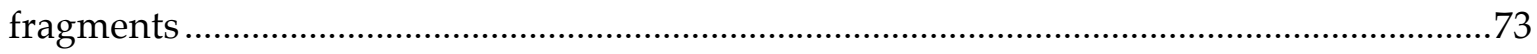

Figure 3-13 Binding behavior of TTD-PHD linker mutants of NP95 I TTD-PHD fragment .75

Figure 3-14 NP95 I TTD-PHD linker insertion reduced binding of UHRF1 TTD-PHD to H3K9me3

Figure 3-15 Binding behavior of TTD-PHD linker mutant of NP95 II TTD-PHD fragment

Figure 3-16 Structural differences between TTD-PHD fragments of NP95 I and NP95 II ..81

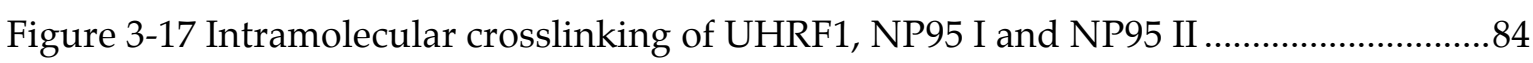

Figure 3-18 Impact of DNA on the H3 tail binding of UHRF1 / NP95 .................................87

Figure 3-19 Co-localization of UHRF1, NP95 I and NP95 II with H3K9me3 in MCF7 cells89

Figure 3-20 Comparison of the nuclear localization of UHRF1 and NP95

Figure 3-21 Retrospective mRNA expression analysis of 17 different types of murine tissues .92

Figure 3-22 Retrospective mRNA expression analysis of different embryonic

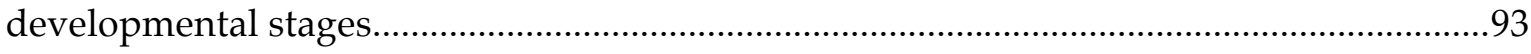

Figure 4-1 Hypothetical models of different conformational states of UHRF1 and NP95..99 Figure 4-2 Regulation of TTD/ linker interactions. 104 


\section{List of Tables}

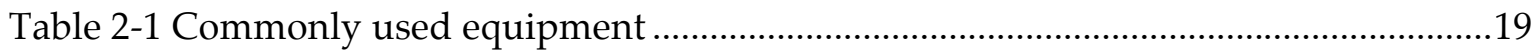

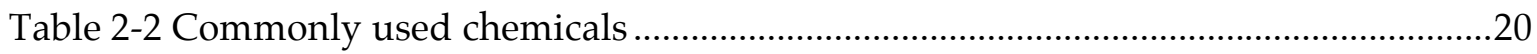

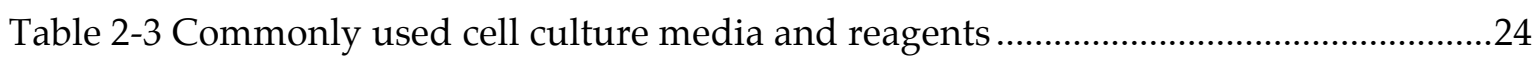

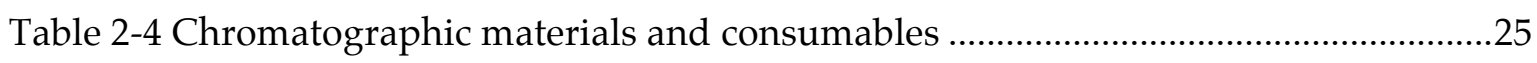

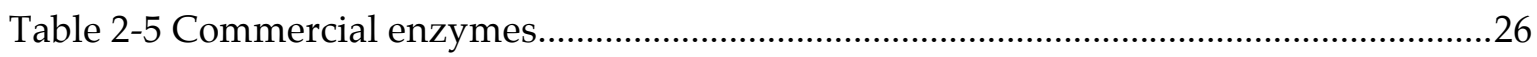

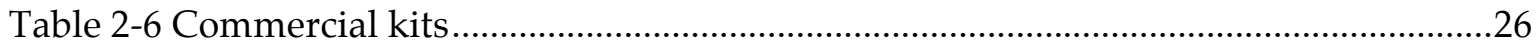

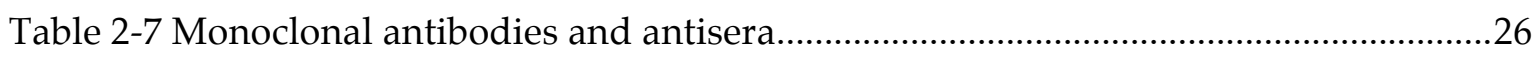

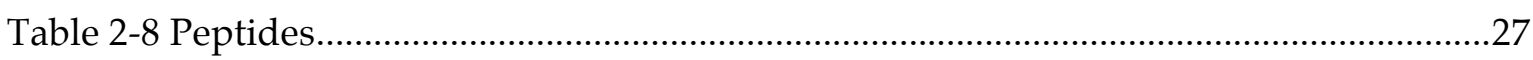

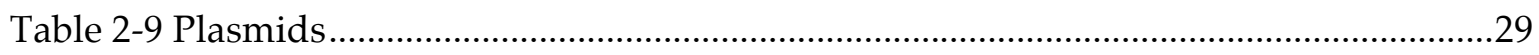

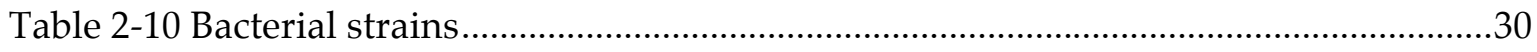

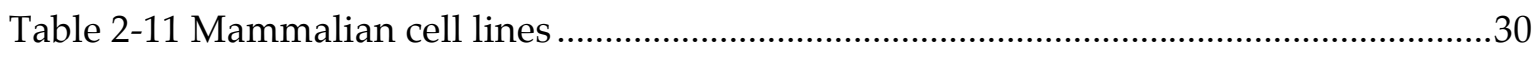

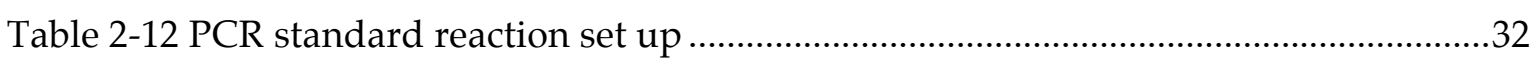

Table 2-13 Molecular weight and molar extinction coefficient of proteins used in this

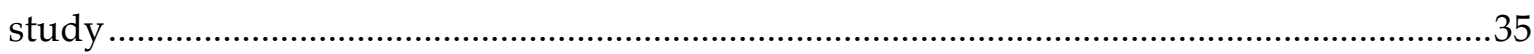

Table 3-1 binding affinity of UHRF1 and PBR-RING to cyclic peptide candidates .............59

Table 3-2 Peptides used for fluorescence polarization and binding domains of UHRF1/ NP95

Table 3-3 binding affinity of all fragments and mutants of UHRF1 and NP95 to indicated peptides 63

Table 3-4 binding affinity of UHRF1 and NP95 to indicated peptides with and without DNA

Table 7-1 Names and sequences of the used cloning primers 130

Table 7-2 Names and sequences of the used sequencing primers 130

Table 7-3 Names and sequences of the used mutagenesis primers 131 


\section{Introduction}

\subsection{Epigenetics and chromatin}

\subsubsection{Epigenetics}

While most cells of a multicellular organism share the same DNA sequence, they vastly differ in their functions and morphologies. This discrepancy between the genotype and the phenotype of different cells was termed by 'epigenotype' by Conrad Hal Waddington in 1942 (Waddington, 2012). The term was used to describe the processes that lead from the uniform genotype to variable phenotypes. The science investigating the process was named 'epigenetics'. In 1958, David Nanney proposed that 'cells with the same genotype may not only manifest different phenotypes, but these differences in expressed potentialities may persist indefinitely during cellular division in essentially the same environment' (Nanney, 1958). This is the origin of our contemporary definition of epigenetics which defines all meiotically or mitotically heritable changes in the cellular phenotype that occur without changing the underlying DNA sequence as epigenetic (Allis et al., 2007). The investigation of these processes in the last few decades has revealed that the molecular basis of these inheritable changes is chromatin.

\subsubsection{The nucleosome}

Chromatin is a polymeric complex of genomic DNA, nuclear proteins and RNA that is necessary to maintain accessibility while the compaction of the eukaryotic genome in in the nuclear compartment (McGinty and Tan, 2015). The basic units of chromatin are the nucleosome core particles, which are connected by a segment of linker DNA. A nucleosome core particle with linker DNA is called a nucleosome, which repeats in the genome every 160 to $240 \mathrm{bp}$ (McGhee and Felsenfeld, 1980).

The nucleosome core particle is formed by the nucleosome core, an octameric complex of two copies each of four core histone proteins, wrapped by $147 \mathrm{bp}$ of DNA in $13 / 4$ superhelical turns (Figure 1-1) (Luger et al., 1997). All core histones share a so-called histone-fold motif in the center of the protein, which is formed by three $\alpha$-helices linked by intervening loops. The histone-fold motif is flanked by $\mathrm{N}$ - and C-terminal extensions (McGinty and Tan, 2015). The core histones interact with each other according to their complementary histone-fold to form heterodimers, $\mathrm{H} 3$ pairs with $\mathrm{H} 4$ and $\mathrm{H} 2 \mathrm{~A}$ pairs with H2B (Figure 1-1B, C). The histone octamer is formed by dimerization of two H3/H4 
heterodimers and the subsequent binding of two $\mathrm{H} 2 \mathrm{~A} / \mathrm{H} 2 \mathrm{~B}$ heterodimers to each half of $(\mathrm{H} 3 / \mathrm{H} 4)_{2}$, through the interaction between the $\mathrm{H} 4$ and $\mathrm{H} 2 \mathrm{~B}$ histone-fold motifs. The surface of the symmetrical core histone octamer bears a strong positive charge and binds to the central $121 \mathrm{bp}$ of nucleosomal DNA. The $13 \mathrm{bp}$ DNA flanking this central region is mainly organized by the two N-terminal histone-fold $\alpha$-helices of $\mathrm{H} 3$ in the octamer (Figure 1-1C, D) (Davey et al., 2002; Luger et al., 1997).

A

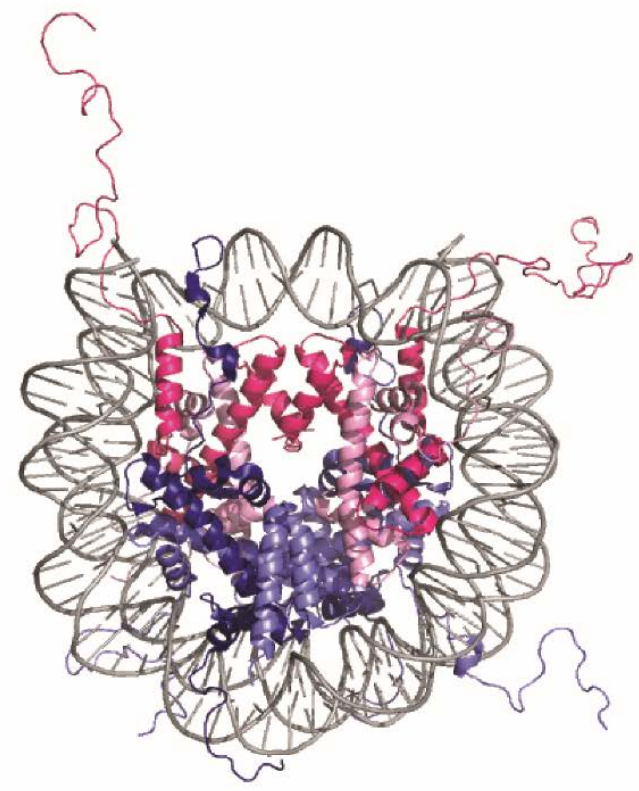

H3

$\mathrm{H} 2 \mathrm{~A}$

$\mathrm{H} 2 \mathrm{~B}$

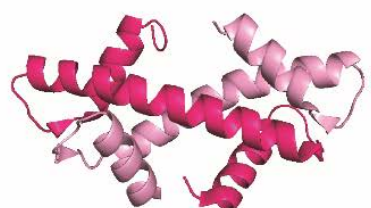

$\mathrm{H} 4$

B

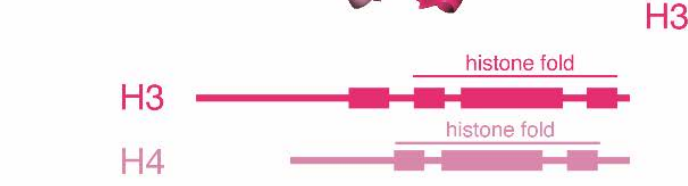

C

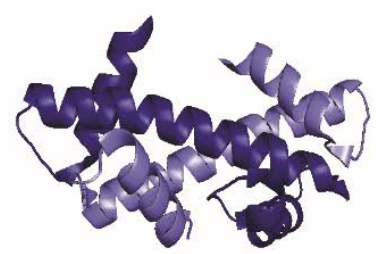

$\mathrm{H} 2 \mathrm{~B}$

$\mathrm{H} 2 \mathrm{~A}$

$\mathrm{H} 2 \mathrm{~A}$

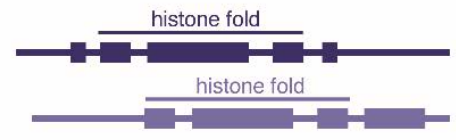

Figure 1-1 The nucleosome core particle structure and the histone-fold heterodimers

(A) Nucleosome core particle structure (PDB ID 1KX5). Histones and DNA are depicted in cartoon and sticks representations, respectively, and colored as indicated. (B) H3/ H4 histone-fold heterodimer. (C) H2A/ H2B histone-fold heterodimer. Structures (top) and schemes (bottom) with secondary structure elements indicated. Based on high-resolution structure in (Davey et al., 2002)(PDB ID 1KX5). Structures were visualized and rendered using MacPyMOL (pymol.org). Graphic was adapted from (McGinty and Tan, 2015).

Apart from the structured regions of the histones that form the histone octamer, the Nterminus of all four core histones comprises a conserved unstructured region, the histone 'tail' domain (Bohm and Crane-Robinson, 1984; Cutter and Hayes, 2015). In nucleosomes under physiological salt conditions, most of these unstructured domains are clinging to the nucleosome body. The 'tail' regions contribute to nucleosome stability and regulate DNA accessibility for binding factors (Ausio et al., 1989; Polach et al., 2000; Wang and Hayes, 2007). Nevertheless, the histone 'tail' domains are subject of extensive posttranslational modifications and a platform for protein interaction with the nucleosome (Jenuwein and Allis, 2001; Zheng and Hayes, 2003). 


\subsubsection{Higher order chromatin structures}

Nucleosomes are linked to one another by a 10-80 bp long segment of linker DNA forming a polynucleosome. The polynucleosome forms a $10 \mathrm{~nm}$ fiber, described as 'beadson-a-string'. This structure is able to compact the DNA five- to ten-fold (Figure 1-2) (Bruce Alberts, 2007). Under physiological salt conditions and in presence of magnesium the 'beads-on-a-string' array is arranged in a compact higher order structure of roughly 30 $\mathrm{nm}$ diameter in vitro (Boule et al., 2015). The structure of this $30 \mathrm{~nm}$ chromatin fiber remains elusive, however, and its existence in vivo in the nucleus of the cell is contentious (Grigoryev and Woodcock, 2012; Joti et al., 2012; Luger et al., 2012; Staynov, 2008). The binding of linker histone H1 (or H5) to the nucleosome and the DNA linker segment stabilizes the $30 \mathrm{~nm}$ fiber (Allan et al., 1986; Hansen, 2002). The higher order structures of chromatin beyond the $30 \mathrm{~nm}$ fiber are little understood to date. The formation of these structures involves looping and long-range contacts of chromatin domains facilitated by non-histone scaffold proteins. One loop contains up to $100 \mathrm{~kb}$ of DNA (Figure 1-2).

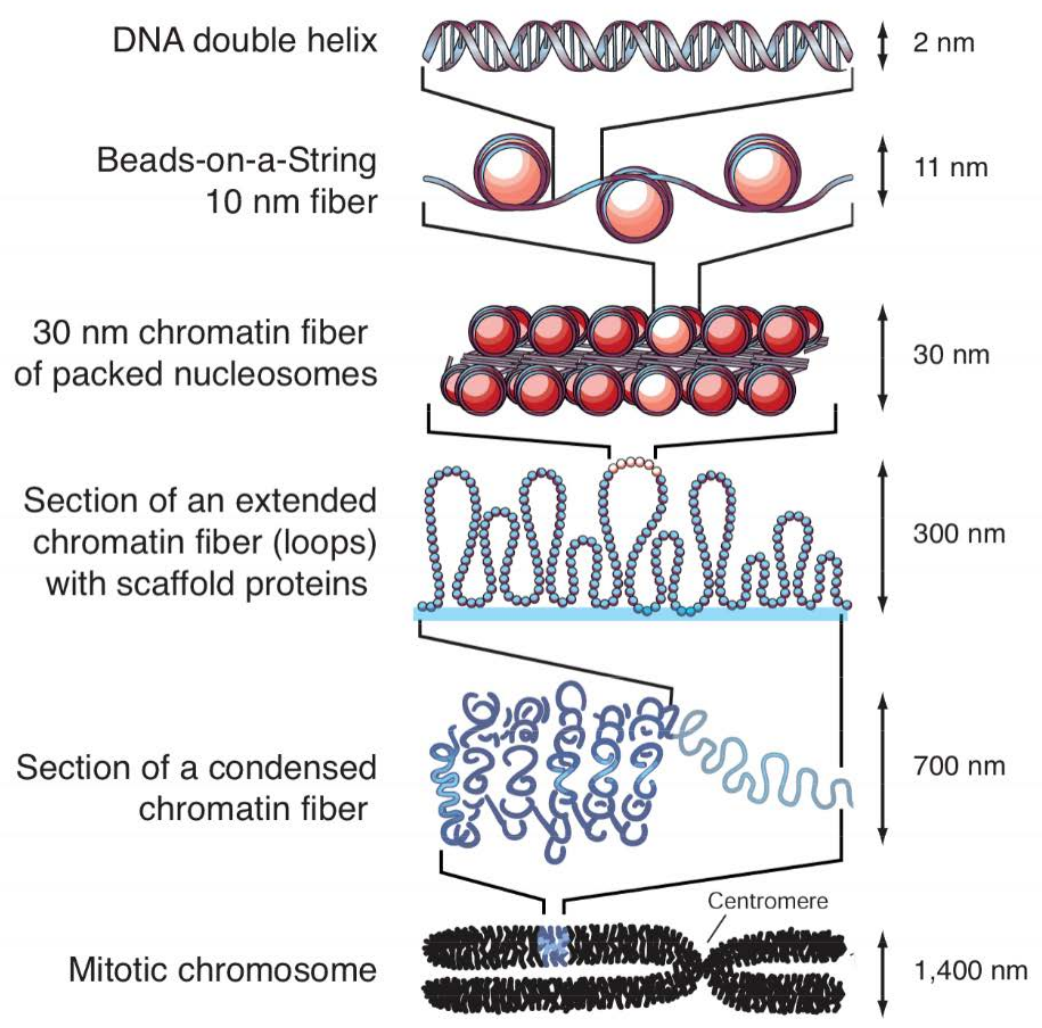

Figure 1-2 Chromatin condensation

The organization of DNA within the chromatin structure is shown. The lowest level of organization is the nucleosome, in which two superhelical turns of DNA (a total of 165 base pairs) are wound around the outside of a histone octamer. Nucleosomes are connected to one another by short stretches of linker DNA. At the next level of organization the string of nucleosomes is folded into a fiber about $30 \mathrm{~nm}$ in diameter, and these fibers are then further folded into higher-order structures. At levels of structure beyond the nucleosome the details of folding are still uncertain. (modified from (Felsenfeld and Groudine, 2003)). 
The degree of chromatin compaction varies throughout the cell cycle. In non-dividing cells, the chromatin structure is generally less condensed, as compared to mitosis when further compaction forms the mitotic chromosome (Cooper and Hausman, 2000; Hames and Hooper, 2000). The described chromatin packaging is important for all cellular processes, since regulatory signals target chromatin, not the DNA. The accessibility of a specific DNA sequence to proteins, such as RNA polymerase or transcription factors, is particularly dependent on the compaction state of the surrounding chromatin structure (Dillon and Festenstein, 2002).

\subsection{Epigenetic modifications}

The formation and maintenance of the chromatin structure is achieved by epigenetic modifications. The classical definition of epigenetic, as described above, includes the heritability of the modification. These modifications were shown to be responsible for the transmission of cellular phenotypes from generation to generation such as gene silencing, $X$ chromosome inactivation and imprinting (Saksouk et al., 2015). Especially the components of the nucleosome, histones and DNA, are subject to a broad variety of heritable chemical modifications. These are associated with transcriptional activity of the underlying genomic regions (Bernstein et al., 2007; Kouzarides, 2007; Li et al., 2007). The modifications include different covalent histone posttranslational modifications and DNA methylation.

\subsubsection{Histone posttranslational modification}

Histone proteins are part of the basic unit of chromatin and are amongst the most conserved proteins in mammalian organisms. Yet, histones contribute largely to the extraordinary plasticity of chromatin structures. Hence, several amino acids either within the histone 'tails', which extend from the center of the nucleosome core, as well as the histone fold part are subject to covalent posttranslational modification.

In the last decades, many of these modifications have been identified including acetylation (Sterner and Berger, 2000), methylation (Zhang and Reinberg, 2001), phosphorylation (Nowak and Corces, 2004), ubiquitylation (Shilatifard, 2006), sumoylation (Nathan et al., 2006), ADP ribosylation (Hassa et al., 2006), deimination (Cuthbert et al., 2004) and propyl isomerization (Nelson et al., 2006). It has been shown that these modifications are not homogenously distributed throughout the genome but are specifically enriched in defined domains. Histone lysine acetylation was identified as a mark of active and less condensed chromatin, and is particularly enriched in the 
promoter and in the $5^{\prime}$ end of coding regions (Millar and Grunstein, 2006). The coding region itself is enriched in lysine trimethylation at histone $\mathrm{H} 3$ lysine 4, 36 and 79 (H3K4me3, H3K36me3, H3K79me3) (Bernstein et al., 2007). In contrast, three different lysine methylation sites are involved in transcriptional silencing and chromatin compaction, H3K9, H3K27 and H4K20 (Kouzarides, 2007). H3K9me3 was shown to play a role in transposon silencing and repression of transcription. H3K27me3 was shown to be essential for X chromosome inactivation in female mammals. H4K20me3 is an important modification in DNA repair processes.

It is generally assumed that each individual modification of a histone leads to a welldefined consequence. Histone modifications function in different ways. First, modifications can recruit a subset of effector proteins equipped with specific modification binding domains. Second, they disrupt the contacts between neighboring nucleosomes by affecting the interactions between histones or histone and DNA (Kouzarides, 2007).

\subsubsection{DNA methylation}

In 1975, R. Holliday and A. D. Riggs suggested that genomic DNA methylation is transmitted in S-phase and might even play a role in gene expression regulation during development (Holliday and Pugh, 1975; Riggs, 1975). Now, we know that DNA methylation maintains lineage-specific expression patterns through continuous cell divisions. In mammals, this mark mostly occurs at carbon-5 of cytosine. 5-Methylcytosine is often referred to as the fifth base of the mammalian genome, reflecting its high abundance. However, the levels of DNA methylation differ between the genomes of different organisms (Lister et al., 2009; Zemach et al., 2010). Remarkably, some genomes, such as those of S. cerevisiae, S. pombe, C. elegans and D. melanogaster do not reveal canonical cytosine methylation (Raddatz et al., 2013).

DNA-methylation is implemented by a group of DNA methyltransferases (Dnmt), including the de novo methylatransferases DNMT3A and DNMT3B as well as the maintenance methyltransferase DNMT1. DNA methylation by these enzymes is catalyzed in the context of CpG motifs. These CpG motifs are symmetrically methylated, so that the methylation mark is present on the sense and antisense strand of DNA, ensuring propagation during replication (Breiling and Lyko, 2015). However, clusters of CpGs, CpG islands, are generally unmethylated. While Dnmts are known to implement DNA methylation, the mechanisms to target these enzymes to specific regions in the genome remain elusive. 
The knock out of DNMT1 and DNMT3 in mouse embryonic stem cells suggested a role of DNA methylation in genomic imprinting, $X$ chromosome inactivation and silencing of transposon sequences (Breiling and Lyko, 2015). DNA methylation was shown to prohibit binding of transcription factors to the DNA as well as to recruit specific 5-methylcytosine binding proteins. Additionally, it is essential for recruitment of chromatin domains to the nuclear lamina (Saksouk et al., 2014). The Dnmts were also found to interact with cotranscriptional modifications (H3K36me) in actively transcribed gene bodies implying a role of DNA methylation in promoting gene transcription. Contrary, promotor regions show a loss of DNA-methylation after binding of transcription factors and upon transcriptional activation (Bestor et al., 2015). Nevertheless, DNA methylation patterns are dynamic and their specific function in the epigenetic regulation of gene expression is not fully understood.

\subsection{Heterochromatin}

\subsubsection{Chromatin domains - Euchromatin and Heterochromatin}

Early cytological studies discerned two different compaction states of chromatin based on their different staining properties for DNA, euchromatin and heterochromatin (Heitz, 1928). Euchromatin is generally less condensed and transcriptionally active. It is gene-rich particularly in housekeeping genes and replicates in early S-phase (Dileep et al., 2015). The predominant fraction of chromatin in interphase nuclei is euchromatin. Only about $10 \%$ of chromatin in interphase nuclei is highly condensed and transcriptionally inactive heterochromatin (Cooper and Hausman, 2000). Heterochromatin was defined as the part of chromatin that remains condensed and is intensively stained by nuclear dyes throughout the cell cycle. It is comprised of few genes, mainly tissue specific genes, and is rich in repetitive sequences and transposable elements (Richards and Elgin, 2002). Heterochromatin is mainly associated with telomeric and pericentric regions in the periphery of the nucleus and replicates in late S-phase.

\subsubsection{Facets of heterochromatin}

In lower eukaryotes, only centromeres and telomeres are constantly heterochromatinized, while the residual genome is in a euchromatic active state (Grewal and Jia, 2007). Increasing genome size and complexity is thought to be responsible for the larger content and growing importance of heterochromatic regions in higher eukaryotes (Bird et al., 1995; Struhl, 1999). The development of electron microscopy and the improvement of 
nuclear staining techniques led to the postulation that heterochromatic regions are partitioned into facultative and constitutive heterochromatin (Brown, 1966).

Facultative heterochromatin was originally described as a developmentally regulated heterochromatinization of tissue specific genes or only one allele of homolog chromosomes (Richards and Elgin, 2002). The most prominent example of facultative heterochromatin is the inactive $\mathrm{X}$ chromosome in female mammals. Facultative heterochromatin is transcriptionally silent and this silent state is heritable during continuous cell divisions. Therefore, it has been defined as an epigenetic chromatin state. Nevertheless it retains the potential for conversion to transcriptionally active euchromatin. Facultative heterochromatin is often associated with Polycomb proteins and H3K27me2/3 as well as histone hypoacetylation (Trojer and Reinberg, 2007). Additionally, H3K9me2 and DNA-methylation were found in inactive alleles (Feil and Berger, 2007).

Similar to facultative heterochromatin, constitutive heterochromatin is transcriptionally silent and this silent state is heritable throughout mitosis and meiosis. Constitutive heterochromatin is mainly formed at regions with very low gene content, next to centromeres, at telomeres and throughout chromosomes. The bulk of constitutive heterochromatin is present at pericentromeric regions (Saksouk et al., 2015). It is usually associated with repetitive sequences as tandem repeats, satellites in size from five up to a few hundred bp, and transposable elements (retrotransposons) (Eymery et al., 2009). Since these repetitive sequences are not conserved between different species or different chromosomes of the same individual, it was suggested that constitutive heterochromatin is epigenetically demarcated. High levels of H3K9me3, H4K20me3, vast histone hypoacetylation and DNA methylation generally characterize constitutive heterochromatin (Dejardin, 2015). These modifications are essential for formation and maintenance of the silent chromatin.

\subsubsection{Maintenance of constitutive heterochromatin}

The silencing of repetitive elements in constitutive heterochromatin is critical for the repression of transposable elements as well as for genome stability and maintenance (Almouzni and Probst, 2011). Thus, the faithful transmission of the epigenetic marks defining constitutive heterochromatin is essential to prevent genome dysfunction. The best-studied example of constitutive heterochromatin is murine pericentromeric heterochromatin. In mice, DNA methylation and H3K9me3 modifications at the pericentromeric heterochromatin are interdependent. Removal of H3K9me3 leads to DNA 
hypomethylation, conversely loss of DNA methylation significantly affects H3K9me3, which finally disrupts pericentromeric architecture (Saksouk et al., 2014).

Heterochromatin protein 1 (HP1) is a highly conserved protein that is implicated in gene silencing and genome stability. In cells, H3K9me3 is bound by the three isoforms of HP1. H3K9me3 in the constitutive heterochromatin is dependent on the histone methyltransferases Suv39h1 and Suv39h2. Both proteins, HP1 and Suv39h1/2, are thought to be part of a self-sustaining loop mechanism to spread heterochromatin features (Maison and Almouzni, 2004). This mechanism involves the interaction of Suv39h1/2 with HP1, which recruits the histone methyltransferase to regions harboring H3K9me3 and leads to propagation of the modification in this region. Additionally, HP1 interacts with the de novo DNA methyltransferase DNMT3B and might be the foundation of DNA methylation at these regions (Lehnertz et al., 2003). In turn, MECP2, a factor binding to methylated DNA, was shown to bind Suv39h1 and might thus stimulate H3K9me3 at sites of DNA methylation (Dejardin, 2015).

DNA methylation maintenance is highly dependent on the function of the maintenance DNA methyltransferase DNMT1 that was shown to specifically methylate hemimethylated CpG motifs generated during replication. DNMT1 is found in a protein complex with histone deacetylases (HDACs) and Ubiquitin-like with PHD and RING Finger domains 1 (UHRF1). UHRF1 is an ubiquitin ligase that is essential for the recruitment of DNMT1 to hemimethylated DNA. It facilitates the maintenance of constitutive heterochromatin by physically linking two essential modifications, hemimethylated DNA and H3K9me3. Loss of UHRF1 compromised not only DNA methylation maintenance but also integrity of pericentromeric regions (Bostick et al., 2007; Sharif et al., 2007).

\subsection{UHRF1}

\subsubsection{Function of UHRF1}

Ubiquitin-like with PHD and RING Finger domains 1 (UHRF1) is a chromatin effector protein that is involved in different chromatin regulating processes like DNA methylation maintenance as well as de novo methylation, histone $\mathrm{H} 3$ methylation and histone $\mathrm{H} 4$ deacetylation (Bostick et al., 2007; Kim et al., 2009; Meilinger et al., 2009; Papait et al., 2007; Sharif et al., 2007; Unoki et al., 2004). It is incorporated in macromolecular complexes with enzymes catalyzing these modifications, DNMT1, DNMT3a/b, HDAC1 and G9a, and is targeting these to defined chromatin loci. It was shown to be an important factor to 
facilitate DNA replication, cell cycle progression, immune cell maturation and DNA damage response (Arima et al., 2004; Jeanblanc et al., 2005; Mistry et al., 2008; Mistry et al., 2010; Obata et al., 2014; Rajakumara et al., 2011; Yao et al., 2016). Recent finding imply a function for UHRF1 as sensor of DNA interstrand crosslinks (Liang et al., 2015; Tian et al., 2015). It is implicated in gene expression regulation, particularly in gene silencing possibly by affecting chromatin structure through histone and DNA methylation (Bronner et al., 2010; Rajakumara et al., 2011; Wang et al., 2012). In addition to that, UHRF1 is a putative oncogenic factor over-expressed in numerous cancers and might be involved in establishment of aberrant DNA methylation and H3K9me3 patterns (Alhosin et al., 2011). It was recently suggested as a novel target for chemotherapeutic drugs (Unoki et al., 2009).

\subsubsection{Sequence conservation of UHRF1 proteins}

UHRF1 proteins are conserved throughout all vertebrate species analyzed to date, while no equivalents are found in D. melanogaster (flybase), C. elegans (wormbase) or S. cerevisiae (yeastgenome). Comparison of UHRF1 amino acid sequences showed a high degree of conservation during the entire vertebrate evolution. The mouse UHRF1 orthologue, known as NP95, has two different splicing variants and shares nearly $77 \%$ sequence identity with the human protein. In contrast to that, in Xenopus leavis, two different genes encode two splicing variants. Both share $68.3 \%$ and $66.8 \%$ sequence identity with the human UHRF1, respectively. Interestingly, the five known folded domains of UHRF1 exhibit very high sequence identity and almost identical predicted secondary structures throughout different vertebrate species, arguing for their essential role in protein function (Bronner et al., 2007).

\subsubsection{UHRF1 - Domain structure and function}

The five conserved domains of UHRF1 comprehend an ubiquitin-like domain often referred to as UBL. Walker and colleagues 2005 showed for NP95 that this domain has the classic $\alpha / \beta$ ubiquitin fold and comprises the structurally conserved surface lysines K31 and $\mathrm{K} 50$, which are putative targets of mono- or poly-ubiquitination and therefore might regulate protein function and/or proteasomal protein turnover (Figure 1-3; pdb entry 2FAZ, not published). However, the role of the UBL domain in context of UHRF1 is not yet fully understood.

UHRF1 is additionally endowed with a Really Interesting and New Gene (RING) domain that has E3 ubiquitin ligase activity. The crystal structure of this domain reveals that it is 
constructed by a zinc-finger, coordinated by two zinc atoms, and a novel unique $\alpha$-helix bundle structure, formed by three helices upstream and one helix downstream of the Znfinger (Figure 1-3; pdb entry 3FL2, not published). A recent report suggests that the RING domain is involved in histone $\mathrm{H} 3$ lysine 23 (H3K23) ubiquitination during S-phase as a prerequisite of successful recruitment of maintenance DNA methyltransferase DNMT1 (Nishiyama et al., 2013).
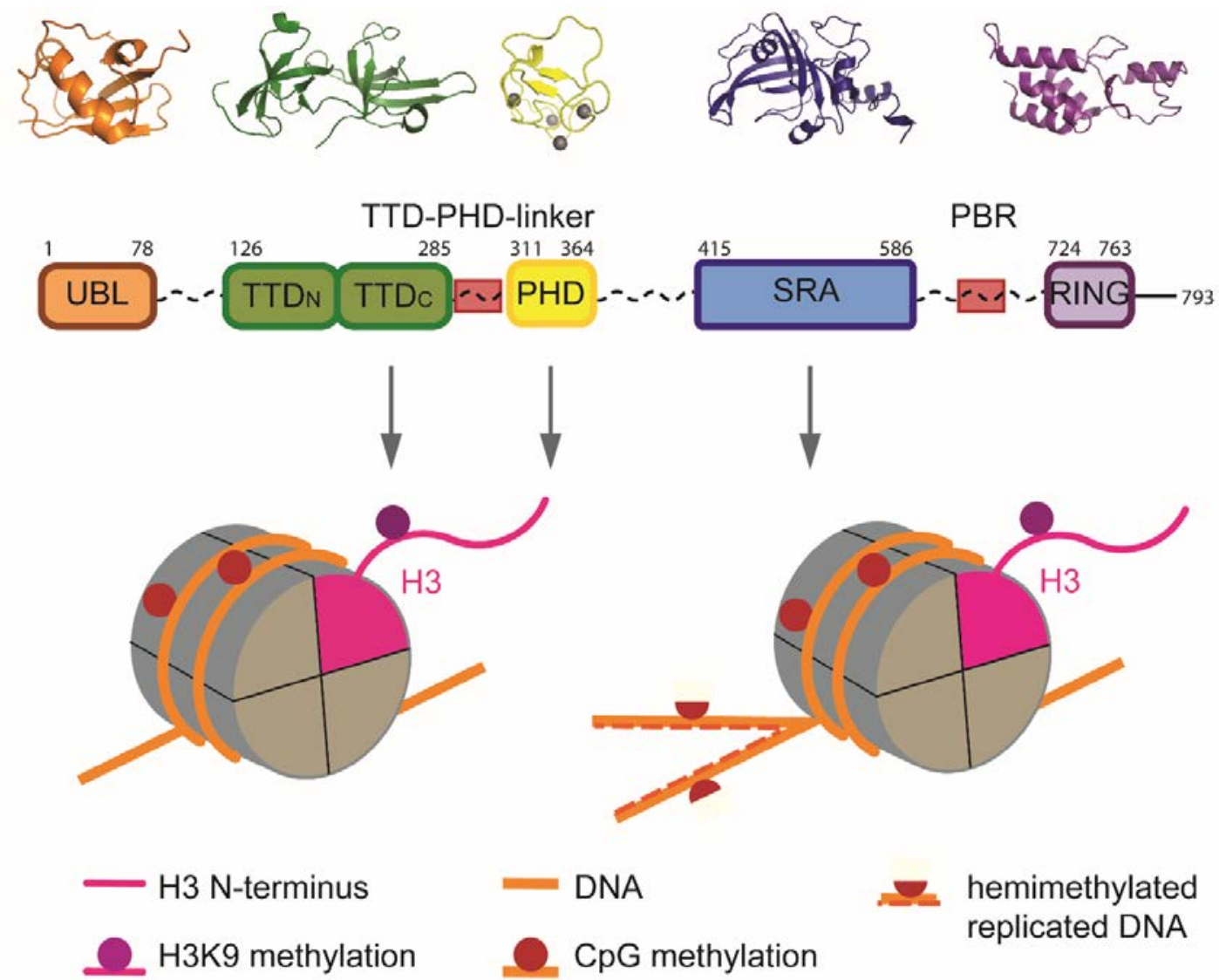

Figure 1-3 UHRF1 is a multi-domain factor with several conserved protein motifs and linker regions Schematic representation of UHRF1 domain organization is shown (UniProtKB: Q96T88). (Top) The structures of the individual domains of UHRF1 as determined by X-ray crystallography and NMR spectroscopy visualized and rendered using MacPyMOL v 1.7.0.3 (pymol.org). (Middle) Domain boundaries are given by respective starting and ending amino acid positions. TTD-PHD linker and PBR are indicated (red boxes). (Botom) Chromatin ligands of the different domains of UHRF1 are shown. Schematic representation of a single nucleosome in interphase (left) and immediately after replication in S-phase (right) are displayed. For simplicity only one H3-tail is shown. UBL, ubiquitin like; TTD, tandem tudor domain; PHD, plant homeodomain; SRA, SET and RING associated; PBR, polybasic region; RING, really interesting and new gene. pdb entries are: UBL, 2FAZ; TTD $:$ TTD $_{c}$, 3DB3; PHD, 3SHB; SRA, 2PB7; RING, 3FL2, modified from (Tauber and Fischle, 2015).

Furthermore, UHRF1 comprises three domains, which allow its accurate localization to specific chromatin regions by facilitating the binding to the modified H3-tail as well as methylated DNA. There is a tandem tudor domain (TTD) C-terminal of the UBL domain, which is composed of two individual tudor domains (TTDN:TTDC) both showing a typical tudor family five-stranded $\beta$-barrel fold. This domain binds to methyl-lysine, specifically 
di- or trimethylated lysine 9 of histone $\mathrm{H} 3$ (H3K9me2/3), between its $\mathrm{N}$ - and C-terminal tudor domains by an aromatic cage that is formed by residues F152, Y188 and Y191 of $\mathrm{TTD}_{\mathrm{N}}$. Interestingly, the isolated TTD binding to H3K9me3 peptide is not sensitive to adjacent serine 10 phosphorylation (H3S10ph), while Lysine 4 trimethylation (H3K4me3) and threonine 6 phosphorylation (H3T6ph) impair its peptide affinity, by disrupting the interaction of $\mathrm{K} 4$ and $\mathrm{T} 6$ side chain and backbone residues with the peptide binding groove between TTDN and TTDc (Figure 1-3) (Nady et al., 2011).

The proximate plant homeodomain (PHD) is a $\mathrm{Zn}$-finger domain; accordingly three zinc atoms coordinate its rod-shape structure. The crystal structure of the PHD with bound H3 peptide revealed that the first two residues of the H3-tail are anchored to the domain with alanine 1 (H3A1) building hydrogen bonds with P353 and E355, while arginine 2 (H3R2) interacts with M332, D334 and D337 (Figure 1-3) (Lallous et al., 2011). The PHD domain is known to recognize this very N-terminus of the H3-tail solely if the H3R2 is unmodified (Rajakumara et al., 2011). Remarkably, the first zinc atom coordinates a loop, which precedes the canonical PHD and is referred to as prePHD. This feature is not found in other described histone binding PHD domains and its detailed function still needs to be determined (Hu et al., 2011; Lallous et al., 2011). It was predicted that the prePHD is essential for the right orientation of the C316, which makes contact with H3K4 (Lallous et al., 2011). However, the PHD alone possesses a very low selectivity towards a specific methylation status of H3K4.

The C-terminally following SET and RNG-associated domain (SRA) is a DNA binding domain, which is constructed of a $\beta$-barrel flanked by $\alpha$-helical elements (Figure 1-3) (Avvakumov et al., 2008) forming a half moon-like structure with a basic inner surface. Two loops sticking out of this structure grasp into the major and the minor groove of the DNA helix and are therein stabilized by V446 and R491 side chains making van der Waals contacts. The R491 is part of the so-called NKR finger, which specifically forms hydrogen bonds with $\mathrm{CpG}$ in the DNA sequence. These contacts flip out a cytosine from the double helix and place it in a binding pocket, which is tailored for the recognition of 5methylcytosine $(5 \mathrm{mC})$ and therefore generates SRA specificity for methylated DNA. In this pocket the methylcytosine is sandwiched by stacking interactions with two aromatic residues (Y478, Y466) and additionally forms hydrogen bonds with D469, T479 and S481 (Avvakumov et al., 2008; Hashimoto et al., 2009). Interestingly, the SRA domain exhibits a great specificity for hemi-methylated DNA. This is facilitated by N489, which is a part of the NKR finger and makes contacts to the non-methylated adverse cytosine on the second DNA strand. Methylation of this cytosine disturbs positioning of the NKR- finger and therefore impairs the SRA binding (Avvakumov et al., 2008). 
All these three separate domains might bind to a specific target in isolation or collaborate with either one of the chromatin interacting domains or both and thereby facilitate a joint readout and crosstalk of histone and DNA modifications. In cells, UHRF1 is preferentially localized to pericentromeric heterochromatin during mid and late S-phase of the cell cycle (Papait et al., 2007; Sharif and Koseki, 2011). It is known to specifically interact with H3K9me3 and DNA methylation in silent chromatin. This was shown to be essential for the maintenance of pericentromeric heterochromatin (Nishiyama et al., 2013). However, it can also be found in euchromatic regions (Karagianni et al., 2008; Liu et al., 2013; Rajakumara et al., 2011), where it is recruited to loci comprising DNA methylation and unmodified histone H3 arginine 2 (H3R2me0) (Rajakumara et al., 2011). How the specific localization of UHRF1 to chromatin loci is regulated during cell cycle and changing cellular environment remains elusive.

\subsubsection{TTD-PHD interplay in recognizing the H3-tail}

Different biophysical studies have quantified the interaction strength of the isolated TTD of UHRF1 with H3K9me3 peptides. Depending on the experimental conditions the dissociation constant $(\mathrm{Kd})$ was determined within a range of $1.0 \mu \mathrm{M}$ to $2.5 \mu \mathrm{M}$ (Gelato et al., 2014; Rothbart et al., 2012) et (Cheng et al., 2013) (Arita et al., 2012). Similarly, binding of the isolated PHD domain to the unmodified N-terminus of $\mathrm{H} 3$ was mapped at a $\mathrm{Kd}$ of $0.7 \mu \mathrm{M}$ to $2.5 \mu \mathrm{M}$ (Arita et al., 2012; Hu et al., 2011; Rajakumara et al., 2011; Xie et al., 2012). In contrast, a UHRF1 TTD-PHD fragment showed around 5-fold enhanced binding (Kd between $0.15 \mu \mathrm{M}$ and $0.5 \mu \mathrm{M}$ ) to a histone $\mathrm{H} 3$ peptide containing both, an unmodified $\mathrm{N}$-terminus of $\mathrm{H} 3$ and the K9me3 mark, implying a multivalent binding mode (Arita et al., 2012; Cheng et al., 2013; Gelato et al., 2014; Rothbart et al., 2013).

Since the H3-tail is embedded in the peptide-binding groove of the TTD in the isolated structure of this complex, how can the PHD get access to the ultimate N-terminus of H3 in the context of the TTD-PHD cassette? Fluorescence/Förster Resonance Energy Transfer (FRET) experiments revealed a conformational shift induced in the TTD-PHD cassette upon interaction with the H3-tail (Cheng et al., 2013). In agreement, structural studies using co-crystalization as well as NMR measurements indicate that the H3-binding mode of the TTD-PHD cassette is different from that of the isolated TTD. Obviously, the short region connecting TTD and PHD (TTD-PHD linker) replaces the H3-tail from the peptidebinding groove of the TTD and itself occupies this interface. The resulting arrangement has the H3-tail connecting the PHD, which binds the ultimate N-terminus, with the TTD, which binds K9me3. Two arginine residues, a lysine and a serine residue (R295-R296K297-S298) in TTD-PHD linker are crucial for stabilizing this TTD-PHD conformation. 
Indeed, the mutation of R295 and R296 resulted in loss of multivalent binding (Arita et al., 2012; Rothbart et al., 2013). In contrast, dynamic NMR studies indicate multiple modes of PHD linkage in relation to TTD-PHD linker and TTD (Rothbart et al., 2013). Also, the TTD-PHD fragment only crystalized in presence of the H3K9me3-tail peptide (Arita et al., 2012). The findings imply that the two domains do not directly interface but that their relative localization is variable without ligand (Tauber and Fischle, 2015).

\subsubsection{Control of TTD/H3K9me3 interaction by a C-terminal polybasic region}

While reductionistic approaches studying isolated domains and combinations thereof have provided detailed insights into the chromatin interaction potential of UHRF1, other studies looked directly at the full-length protein. Here, striking differences in interaction specificity for the H3-tail were observed. While native UHRF1 analyzed in the context of mammalian cell extracts shows clear preferences for H3K9me3, the recombinant protein expressed in bacteria or insect cells binds similarly to unmodified H3 and H3K9me3 peptides (Gelato et al., 2014; Karagianni et al., 2008; Liu et al., 2013; Rajakumara et al., 2011; Rottach et al., 2010). We recently showed that this discrepancy is due to intramolecular interaction of the TTD with a polybasic region (PBR) located between the SRA and RING domains of UHRF1 (Figure 1-3) (Gelato et al., 2014). Our studies imply that in the absence of ligands (i.e. the recombinant purified protein) the PBR but not TTDPHD linker occupies the peptide-binding groove of the TTD. This prevents interaction with H3K9me3, whereas the PHD is unaffected and can bind the ultimate unmodified H3tail. NMR experiments have revealed that a K-R-K-S motif (K648-R649-K650-S651) is essential for placing the PBR in the peptide-binding groove of the TTD surface. Competition experiments with isolated domains indicate that this interaction is stronger than the similar TTD/TTD-PHD linker interplay. It also fully blocks the binding of the H3K9me3-tail. In the context of the recombinant, full-length protein mutagenesis of the KR-K-S motif is necessary to release the PBR from the TTD (Gelato et al., 2014). The resulting mutant UHRF1 protein appears to be in an intermediate state as the TTD and PHD both can bind their respective $\mathrm{K} 9 \mathrm{me} 3$ and $\mathrm{H} 3$ unmodified $\mathrm{N}$-terminus ligands (Tauber and Fischle, 2015).

Dialysis of recombinant UHRF1 against nuclear extract isolated from HeLa cells induced yet another UHRF1 binding state. Interestingly, this form resembles the native cellular protein in preference for $\mathrm{H} 3 \mathrm{~K} 9 \mathrm{me} 3$ over $\mathrm{H} 3 \mathrm{~K} 9 \mathrm{me} 0$ (Gelato et al., 2014). The results infer an allosteric regulatory mode of UHRF1 that is conducted by small nuclear factors. 


\subsection{Nuclear PIPs}

Since gene expression patterns change in response to transient changes in cellular physiology (e.g. external stress stimuli), it has been suggested that small signaling molecules can have a direct effect on chromatin (Lu and Thompson, 2012). In this context, Phosphatidylinositol phosphates (PIP) are particularly interesting, because a pool of these lipids was found in the nucleus that is independently regulated from the membranebound cytoplasmic fraction in response to extracellular and/or intracellular stimuli. PIPs are amphiphilic glycerophospholipids that consist of a polar inositol head group linked by a phosphodiester bridge to a glycerol backbone branching two non-polar fatty acids. A great fraction of nuclear phospholipids is isolated together with chromosome associated nonhistone proteins (Manzoli et al., 1976) and further analysis revealed that lipids could thus be associated with hetero- and euchromatin (Rose and Frenster, 1965).

\subsubsection{PI(5)P}

$\mathrm{PI}(5) \mathrm{P}$ is a low abundance PIP present primarily in mammalian cytoplasmic cell membranes with a smaller population in the cell nucleus (Figure 1-4A) (Pendaries et al., 2005). While its nuclear functions are not fully clear, its presence in this compartment is highly conserved throughout eukaryotes (Balla et al., 2012; Barlow et al., 2010; Clarke et al., 2001). The levels of cytosolic and nuclear PI(5)P are modulated spatially and temporally in response to physiological and pathological stimuli, such as cell cycle progression signals, oxidative stress, UV radiation, cancer formation and dehydration in plants (Clarke et al., 2001; Divecha et al., 1991; Jones et al., 2006; Jones et al., 2013; Keune et al., 2012; Shisheva, 2013).

\subsubsection{Phosphoinositol dependent regulation of chromatin factors}

PI(5)P was shown to be a physiological ligand of several chromatin associated proteins. The best understood example is the tumor suppressor inhibitor of growth 2 (ING2). The structure of ING2 comprises a PHD domain, which specifically interacts with H3K4me3, the hallmark for actively transcribed genes. ING2 is part of a chromatin modifying complex comprising Sin3A and HDAC1 that targets gene promoter regions, where it leads to histone deacetylation and thus to transcriptional silencing (Shi et al., 2006). In vitro studies revealed that ING2 interacts with PI5P via basic residues in its PHD domain as well as via an adjacent polybasic patch. This interaction was shown to facilitate ING2 chromatin association in vivo and thereby affect gene transcription (Figure 1-4B) (Gozani et al., 2003; Jones et al., 2006). Gozani and colleagues (Gozani et al., 2005) could show that 14 
ING2 localization in BalbC-3T3 cells in response to the treatment with exogenous PI5P undergoes rapid changes. Zhao and colleagues (Zhao et al., 1998) made similar observations.

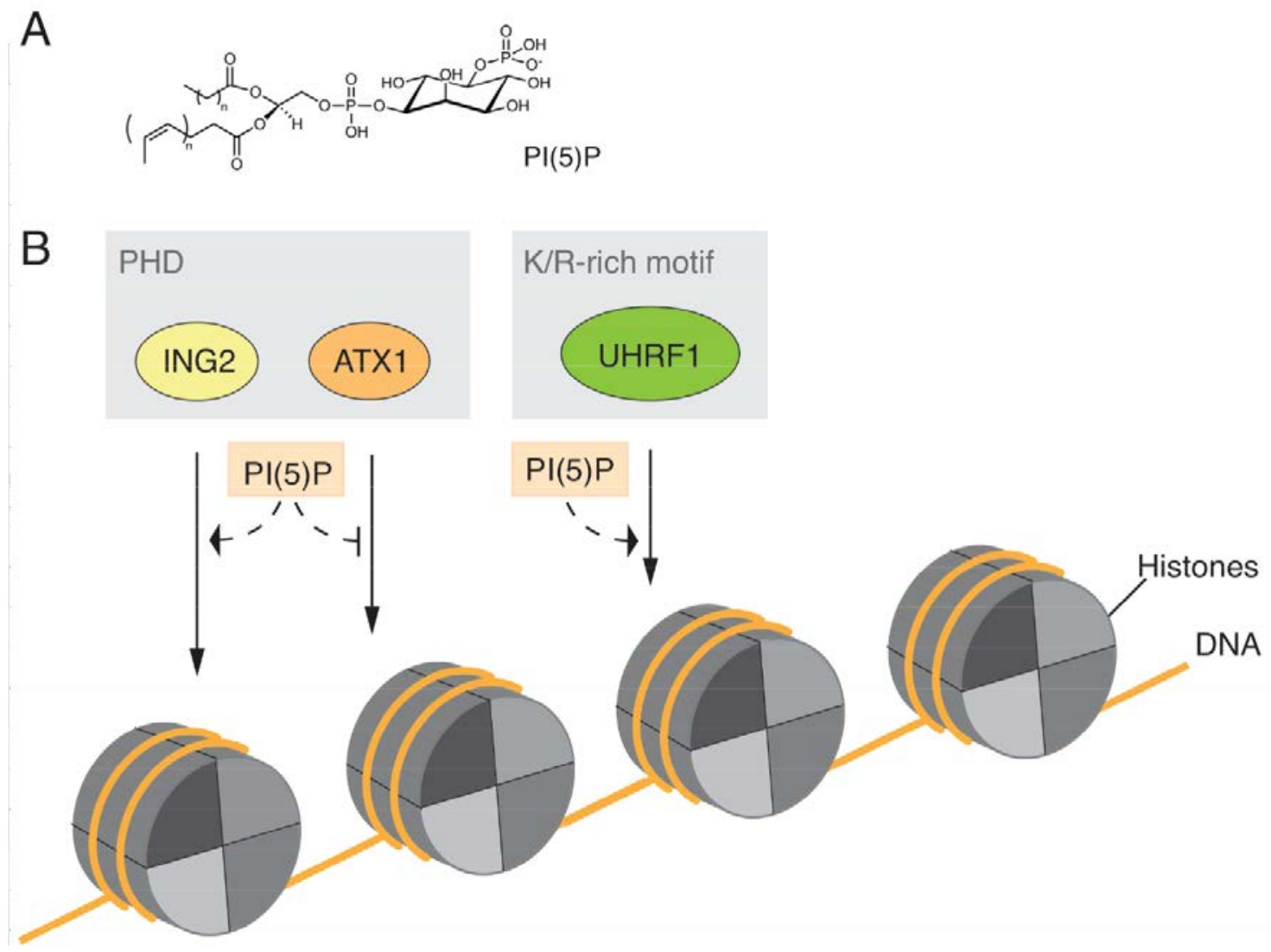

Figure 1-4 Regulation of chromatin interaction of several known factors by PIs

(A) Structure of PI(5)P with the C5 position phosphorylated and the phosphodieester linkage to the dialcylglycerole. (B) Chromatin interacting factors and their PIP interaction motif are shown. ING2 and ATX bind PIP via a PHD domain. UHRF1 and TopoII $\alpha$ via a R/K rich PIP consensus sequence. ATX1 binding to chromatin is blocked by interaction with PIs, while UHRF1 and ING2 binding affinity/specificity is changed or enhanced. For simplicity, chromatin is represented by four nucleosomes. Histones (grey) and DNA (yellow) are indicated

In A. thaliana another factor, ATX1, was shown to interact with PI5P through its PHD finger. In contrast to ING2 binding of PI5P inhibits chromatin association and leads to cytoplasmic localization of ATX1 (Figure 1-4B) (Alvarez-Venegas et al., 2006; Ndamukong et al., 2010). This indicates that a PHD domain with an accompanied polybasic patch is an important platform for PI binding and its subsequent manipulation of effector functionality. Unfortunately it is not known until now how PI5P affects chromatin association via the PHD domain of these factors. 


\subsubsection{Regulation of UHRF1 TTD/ PBR interaction by PI(5)P}

We previously showed that allosteric ligands provide a level of regulating UHRF1 conformational states. Based on our observations that recombinant UHRF1 behaved differently from cellular protein but could be converted to a state with similar H3K9me3-binding properties after dialysis against nuclear extract, we biochemically defined the cofactor and regulator as PI(5)P (Figure 1-4A, B) (Gelato et al., 2014). We could show that PI(5)P binding to UHRF1 specifically requires the PBR region and that this interaction releases the latter from the peptide-binding groove of the TTD. In consequence, a conformation of UHRF1 is established that allows the TTD to bind H3K9me3 independent of the PHD (Gelato et al., 2014). 


\subsection{Aims of the thesis}

Since UHRF1 is recruiting chromatin modifying enzymes to specific loci, the right localization of the protein is of great importance to facilitate downstream events affecting the chromatin structure and function. The aim of this thesis is to gain insight in the interaction of UHRF1 proteins with chromatin modifications. In this context, I focus on the regulation of UHRF1 by flexible inter-domain linker regions.

In particular, I have the following objectives:

- To investigate the role of the PBR region in UHRF1 nuclear localization

- To investigate the influence of changing nuclear PI(5)P levels on the nuclear localization of UHRF1

- To identify novel small molecule regulators of UHRF1

- To investigate whether the inter-domain linker dependent regulation is conserved in murine NP95

- To investigate the influence of different inter-domain linker regions on the nuclear localization of UHRF1 and NP95 



\section{Material and Methods}

\subsection{Materials}

\subsubsection{Laboratory equipment}

Commonly used laboratory equipment is listed in table 2-1.

Table 2-1 Commonly used equipment

\begin{tabular}{ll}
\hline Equipment & Supplier \\
\hline XS1 & Mettler-Toledo, Giessen \\
Kern EG & Kern \& Sohn, Balingen \\
Centrifuge Sorvall Evolution RC & Thermo Scientific, Braunschweig \\
Centrifuges 5415R/ 5810R/5424 & Eppendorf, Hamburg \\
EmulsiFlex-C5 High Pressure Homogenizer & Avestin, Ottawa (CA) \\
FACS Canto II & BD Biosciences, Heidelberg \\
Gel documentation system ChemiDoc MP & Bio-Rad, München \\
Gene Pulser II & Bio-Rad, München \\
Heraeus HERAcell 240 Incubator & Thermo Scientific, Braunschweig \\
Heraeus HERAsafe Biol. safety cabinet & Thermo Scientific, Braunschweig \\
Axiovert 40CFL (HBO50/ AC) & Carl Zeiss, Jena \\
TCS SP5 & Leica, Wetzlar \\
Mini-Protean gel casting system & Bio-Rad, München \\
MiniTrans-Blot & Bio-Rad, München \\
Multitron shaker & HT Infors, Braunschweig \\
NanoDrop ND-1000 & Peqlab, Erlangen \\
PCR Mastercycler epgradient S & Eppendorf, Hamburg \\
pH Meter & Mettler-Toledo, Giessen \\
PlateChameleon & Hidex, Turku (FI) \\
Infinite M1000 Pro & Tecan, Männedorf (CHE) \\
Power supply Power Pack basic & Bio-Rad, München \\
SpeedVac Savant SPD131DDA & Thermo Scientific, Braunschweig \\
Stuart Gyrorocker SSL3 & Sigma, Steinheim \\
Sub-Cell-GT Agarose gel electrophoresis & Bio-Rad, München \\
Thermomixer comfort & Eppendorf, Hamburg \\
Waterbath TW12 & Julabo, Seelbach \\
\hline & \\
\hline &
\end{tabular}




\subsubsection{Chemicals}

Commonly used chemicals are listed in table 2-2.

Table 2-2 Commonly used chemicals

\begin{tabular}{|c|c|}
\hline Chemical & Supplier \\
\hline 2-Mercaptoethanol & Sigma, Steinheim \\
\hline 2X YT medium & Roth, Karlsruhe \\
\hline 4,6-Diamidin-2-phenylindol (DAPI) & Thermo Scientific, Braunschweig \\
\hline Acetic acid & Merck, Mannheim \\
\hline Acrylamide / Bisacrylamide solution (37.5:1) & Merck, Mannheim \\
\hline Agar & Roth, Karlsruhe \\
\hline Agarose & Serva, Heidelberg \\
\hline Ammonium persulfate (APS) & AppliChem, Darmstadt \\
\hline Ampicillin & AppliChem, Darmstadt \\
\hline Bovine serum albumin & Sigma, Steinheim \\
\hline Bromophenol Blue & Serva, Heidelberg \\
\hline BS3 protein crosslinker & Thermo Scientific, Braunschweig \\
\hline Calcium chloride & Roth, Karlsruhe \\
\hline Chloramphenicol & Amresco, Solon (USA) \\
\hline Chloroform & Merck, Mannheim \\
\hline Coomassie Brilliant Blue & Bio-Rad, München \\
\hline $\begin{array}{l}\text { Deoxynucleoside-5'-triphosphate (dATP, dCTP, } \\
\text { dGTP, dTTP) }\end{array}$ & Roth, Karlsruhe \\
\hline Dimethylsulfoxid (DMSO) & Sigma, Steinheim \\
\hline Di-sodium-hydrogen-phosphate & AppliChem, Darmstadt \\
\hline Dithiothreithol (DTT) & Merck, Mannheim \\
\hline Doxicyclin & Sigma, Steinheim \\
\hline Ethanol & Roth, Karlsruhe \\
\hline Ethidium bromide solution & Roth, Karlsruhe \\
\hline Ethylenediamine tetraacetate (EDTA) & Merck, Mannheim \\
\hline Formaldehyde & Sigma, Steinheim \\
\hline D-(+)-Glucose-monohydrate & Merck, Mannheim \\
\hline Glycerol & Merck, Mannheim \\
\hline Isopropyl- $\beta$-D-thiogalacto-pyranoside (IPTG) & AppliChem, Darmstadt \\
\hline Imidazole & Roth, Karlsruhe \\
\hline Kanamycin & Amresco, Solon (USA) \\
\hline LB Broth & MOBIO, Hamburg \\
\hline Magnesium chloride & Merck, Mannheim \\
\hline
\end{tabular}


Magnesium sulfate

Methanol

Milk powder

$\mathrm{N}-2-\mathrm{Hyd}$ roxyethylpiperazine-N'-2ethansulphonic acid (HEPES)

Nocodazole

Nonidet P-40 (NP-40)

Phenylmethanesulfonyl fluorid (PMSF)

Phenol/Chloroform/Isoamylalcohol

Ponceau S

Potassium chloride

Potassium-di-hydrogen-phosphate

Potassium hydroxide

Propidium iodide

Rotiphorese Gel 40

Rotiphorese Gel A

Rotiphorese Gel B

Sodium chloride

Sodium dodecyl sulfate (SDS), ultra pure

Sodium hydroxide

Tetramethylethylendiamine (TEMED)

Tetracycline

Thymidine

Tris(hydroxymethyl)aminomethane (Tris base)

Triton X-100

Tryptone/ Peptone

Tween20

Water (steril)

Yeast extract
Roth, Karlsruhe

Merck, Mannheim

Régilait, Saint-Martin-Belle-Roche (FR)

VWR, Poole (UK)

Sigma, Steinheim

Sigma, Steinheim

Sigma, Steinheim

Roth, Karlsruhe

Sigma, Steinheim

Merck, Mannheim

Merck, Mannheim

Merck, Mannheim

Roth, Karlsruhe

Roth, Karlsruhe

Roth, Karlsruhe

Roth, Karlsruhe

Merck, Mannheim

Roth, Karlsruhe

Merck, Mannheim

Sigma, Steinheim

Roth, Karlsruhe

Sigma, Steinheim

Roth, Karlsruhe

Merck, Mannheim

Roth, Karlsruhe

Sigma, Steinheim

Sigma, Steinheim

MOBIO, Hamburg

\subsubsection{Phosphatidylinositol phosphates}

All phosphatidylinositol phosphates were purchased from Echelon Biosciences Inc., Salt Lake City (USA).

Phosphatidylinositol-4-phosphate

Phosphatidylinositol-5-phosphate
Catalog No.: P-4016

Catalog No.: P-5016 


\subsubsection{Commonly used buffers and solutions}

10X DNA loading dye

$10 X$ PBS

6X SDS loading buffer

10X SDS running buffer

10X TBE

Coomassie staining solution

Destaining solution

DNA hybridization buffer

$\begin{aligned} 30 \%[\mathrm{v} / \mathrm{v}] & \text { Glycerol } \\ 10 \mathrm{mM} & \text { EDTA } \\ 0.25 \%[\mathrm{w} / \mathrm{v}] & \text { Bromophenol blue }\end{aligned}$

$\begin{array}{rl}1.37 \mathrm{M} & \mathrm{NaCl} \\ 27 \mathrm{mM} & \mathrm{KCl} \\ 100 \mathrm{mM} & \mathrm{Na} 2 \mathrm{HPO} 4 \\ 20 \mathrm{mM} & \mathrm{KH}_{2} \mathrm{PO}_{4}\end{array}$

$375 \mathrm{mM} \quad$ Tris- $\mathrm{HCl}$ pH 6.8

$12 \%$ SDS

$60 \%$ Glycerol

$0.6 \%$ Bromphenol blue

$1.5 \mathrm{M}$ 2-Mercaptoethanol

$\begin{aligned} 250 \mathrm{mM} & \text { Tris base } \\ 1.92 \mathrm{M} & \text { Glycine } \\ 1 \%[\mathrm{w} / \mathrm{v}] & \text { SDS }\end{aligned}$

$890 \mathrm{mM} \quad$ Tris base

$890 \mathrm{mM} \quad$ Boric acid

$20 \mathrm{mM}$ EDTA-NaOH $\mathrm{pH} 8.0$

$0.1 \%[\mathrm{w} / \mathrm{v}] \quad$ Coomassie Brilliant Blue R250

$10 \%[\mathrm{v} / \mathrm{v}] \quad$ Acetic acid

$50 \%$ [v/v] Methanol

$10 \%[\mathrm{v} / \mathrm{v}] \quad$ Acetic acid

$7.5 \%[\mathrm{v} / \mathrm{v}] \quad$ Methanol

$\begin{array}{rl}10 \mathrm{mM} & \text { Tris } \mathrm{pH} 7.5 \\ 50 \mathrm{mM} & \mathrm{NaCl} \\ 1 \mathrm{mM} & \text { EDTA }\end{array}$


SDS-PAGE stacking gel

SDS-PAGE resolving gel

$\underline{L B \text { agar plates }}$

Ponceau stain

Propidium iodide stain

in $1 x P B S$

SOC medium

Lysis buffer

$\begin{aligned} 0.68 \mathrm{M} & \text { Tris-HCl pH } 6.8 \\ 0.1 \%[\mathrm{w} / \mathrm{v}] & \text { SDS } \\ 4 \% & \text { Acrylamide:bisacrylamide }(37.5: 1) \\ 0.1 \%[\mathrm{v} / \mathrm{v}] & \text { TEMED } \\ 0.1 \%[\mathrm{w} / \mathrm{v}] & \text { APS }\end{aligned}$

$\begin{aligned} 0.4 \mathrm{M} & \text { Tris-HCl } \mathrm{pH} 8.8 \\ 0.1 \%[\mathrm{w} / \mathrm{v}] & \text { SDS } \\ 10-20 \% & \text { acrylamide:bisacrylamide }(37.5: 1) \\ 0.1 \%[\mathrm{v} / \mathrm{v}] & \text { TEMED } \\ 0.1 \%[\mathrm{w} / \mathrm{v}] & \text { APS }\end{aligned}$

$25 \mathrm{~g} / 1 \quad$ LB broth

$1.5 \%[\mathrm{w} / \mathrm{v}]$ Agar

$5 \%[\mathrm{w} / \mathrm{v}] \quad$ Ponceau S

$1 \%$ [v/v] Acetic acid

$100 \mu \mathrm{g} / \mathrm{ml} \quad$ Propidium iodide

$0.6 \%[\mathrm{v} / \mathrm{v}] \quad \mathrm{NP} 40$

$1 \mathrm{mg} / \mathrm{ml} \quad$ RNase A

$\begin{array}{rl}2 \% & \text { Tryptone } \\ 0.5 \% & \text { Yeast extract } \\ 10 \mathrm{mM} & \mathrm{NaCl} \\ 2.5 \mathrm{mM} & \mathrm{KCl} \\ 20 \mathrm{mM} & \text { Glucose } \\ & \\ 50 \mathrm{mM} & \text { Tris- } \mathrm{HCl} \mathrm{pH} 8.0 / 7.5 \\ 300 \mathrm{mM} & \mathrm{NaCl} \\ 10 \mathrm{mM} & \text { Imidazole pH } 8.0 \\ 1 \mathrm{x} & \text { Protease Inhibitor cocktail (EDTA-free) }\end{array}$


Wash buffer

Elution buffer

Dialysis buffer

$$
\begin{array}{rl}
50 \mathrm{mM} & \text { Tris-HCl pH 8.0/7.5 } \\
500 \mathrm{mM} & \mathrm{NaCl} \\
10 \mathrm{mM} & \text { Imidazole } \mathrm{pH} 8.0
\end{array}
$$

$\begin{array}{rl}50 \mathrm{mM} & \text { Tris- } \mathrm{HCl} \mathrm{pH} 8.0 / 7.5 \\ 100 \mathrm{mM} & \mathrm{NaCl} \\ 300 \mathrm{mM} & \text { Imidazole } \mathrm{pH} 8.0 \\ 5 \%[\mathrm{v} / \mathrm{v}] & \text { Glycerol }\end{array}$

$50 \mathrm{mM} \quad$ Tris- $\mathrm{HCl} \mathrm{pH} 8.0 / 7.5$

$$
150 \mathrm{mM} \quad \mathrm{NaCl}
$$$$
10 \%[\mathrm{v} / \mathrm{v}] \quad \text { Glycerol }
$$

$1 \mathrm{mM} \quad$ DTT

\subsubsection{Cell culture media and reagents}

Commonly used cell culture media and reagents are listed in table 2-3.

Table 2-3 Commonly used cell culture media and reagents

Media/Reagent

0.05\% Trypsin/EDTA, Gibco

DMEM/DMEM+GlutaMax-I, Gibco

DPBS, Gibco

D-Glucose solution (10\%)

HyClone Bovine growth serum

Lipofectamine LTX and Plus reagent

MEM non-essential amino acids

OptiMEM, Gibco

Sodium pyruvate, Gibco

TC-Protector cell freezing medium

\section{Supplier}

Thermo Scientific, Braunschweig

Thermo Scientific, Braunschweig

Thermo Scientific, Braunschweig

Sigma, Steinheim

Thermo Scientific, Braunschweig

Invitrogen, Karlsruhe

Sigma, Steinheim

Thermo Scientific, Braunschweig

Thermo Scientific, Braunschweig

Bio-Rad, München 


\subsubsection{Chromatographic materials and consumables}

Chromatographic materials and consumables are listed in table 2-4.

Table 2-4 Chromatographic materials and consumables

\section{Consumables}

$1 \mathrm{~Kb}$ Plus DNA ladder

6-well TC dish

96 well polystyrene plate, black, flat bottom

Amersham Hyperfilm ECL

Amicon Ultra centrifugal filter devices

(MWCO 3, 10, $30 \mathrm{kDa}$ )

Dynabeads M-280 sheep anti-mouse IgG

HisPur cobalt resin

Kodak BioMax MR film

Myc-tag magnetic bead conjugate

Nitrocllulose membrane

Protease Inhibitor (EDTA-free)

SeeBlue Plus2 prestained protein standard

Slide-A-Lyzer dialysis units (MWCO 3,500 and 10,000; volume $0.5 \mathrm{ml}$ )

Spectra/ Por Dialysis Membrane (MWCO $3,500$ and $6-8,000)$

Streptavidin MagneSphere Paramagnetic Particles

TC Dishes (100/150 standard)

TC serological pipettes cellstar $(5 \mathrm{ml}, 10 \mathrm{ml}$, 25ml)

\section{Supplier}

Invitrogen, Karlsruhe

Corning, Corning (USA)

Corning, Corning (USA)

GE Healthcare, Buckinghamshire (UK)

Millipore, Billerica (USA)

Dynal Biotech / Invitrogen, Karlsruhe

Thermo Scientific, Braunschweig

Carestream Health, New York (USA)

Cell signaling technology, Cambridge (UK)

GE Healthcare, Buckinghamshire (UK)

Roche, Basel (CHE)

Invitrogen, Karlsruhe

Pierce/ Thermo Scientific, Rockford (USA)

Spectrum Laboratories, Rancho Dominguez (USA)

Promega, Mannheim

Sarstedt, Nümbrecht

Greiner Bio-One, Kremsmünster (AT) 


\subsubsection{Commercial enzymes}

Commercial enzymes used for this work are listed in table 2-5.

Table 2-5 Commercial enzymes

\begin{tabular}{ll}
\hline Enzyme & Supplier \\
\hline Antarctic phosphatase & New England Biolabs, Frankfurt \\
DpnI & Fermentas, St. Leon-Rot \\
Micrococcal nuclease & Takara Clontech, Saint-Germain-en-Laye (FR) \\
Pfu Polymerase & Fermentas, St. Leon-Rot \\
PfuUltra II Fusion HS polymerase & Stratagene, La Jolla (USA) \\
Q5 High-Fidelity DNA polymerase & New England Biolabs, Frankfurt \\
Restriction enzymes & New England Biolabs, Frankfurt \\
T4 DNA ligase & New England Biolabs, Frankfurt \\
\hline
\end{tabular}

\subsubsection{Commercial kits}

Commercial kits used in this work are listed in table 2-6.

Table 2-6 Commercial kits

Kit Supplier

ECL Plus Western Blotting Detection

GE Healthcare, Buckinghamshire (UK) System

ECL Western Blotting Detection Reagent GE Healthcare, Buckinghamshire (UK)

NucleoBond Xtra Midi Plus

Machery\&Nagel, Düren

NucleoSpin Plasmid

Machery\&Nagel, Düren

NucleoSpin Gel and PCR clean up

Machery\&Nagel, Düren

\subsubsection{Monoclonal antibodies and antisera}

Monoclonal antibodies and antisera used in this work are listed in table 2-7.

Table 2-7 Monoclonal antibodies and antisera

\begin{tabular}{l|l|l|l}
\hline Reagent & Source & Dilution & Supplier/Cat.No. \\
\hline Primary antibodies & & & \\
$\alpha$-FLAG M2 & mouse, monoclonal & $1 \mu 1(\mathrm{IP})$ & Sigma Aldrich, F1804 \\
\hline
\end{tabular}




\begin{tabular}{|c|c|c|c|}
\hline$\alpha-\mathrm{H} 3$ & rabbit, polyclonal & $1: 10,000(\mathrm{WB})$ & Abcam, ab1791 \\
\hline$\alpha-H 3$ K9me3 & rabbit, polyclonal & 1:1,000 (IF) & Active motif, \#39161 \\
\hline$\alpha$-His6 & mouse, monoclonal & 1:1,000 (WB) & Santa cruz, sc-57598 \\
\hline$\alpha$-myc tag & mouse, monoclonal & $\begin{array}{l}1: 1,000(\mathrm{WB}) \\
1: 500 \text { (IF) } \\
1 \mu 1(\mathrm{IP})\end{array}$ & $\begin{array}{l}\text { Abcam, ab9103 } \\
\text { CST, \#2276 }\end{array}$ \\
\hline$\alpha$-UHRF1 & mouse, monoclonal & $\begin{array}{l}1: 1,000(\mathrm{WB}) \\
1 \mu 1(\mathrm{IP})\end{array}$ & Santa cruz, sc-373750 \\
\hline Secondary antibodie & & & \\
\hline$\alpha$-mouse HRP & goat, polyclonal & $1: 5,000$ & Dako, P0447 \\
\hline$\alpha$-rabbit HRP & swine, polyclonal & $1: 5,000$ & Dako, P0399 \\
\hline$\alpha$-rabbit Alexa 488 & donkey, polyclonal & 1:500/1,000 (IF) & Invitrogen, A21206 \\
\hline$\alpha$-mouse Alexa 546 & goat, polyclonal & $1: 500 / 1,000$ (IF) & Invitrogen, A11003 \\
\hline
\end{tabular}

\subsubsection{Peptides}

Peptides that were used for this work are listed in table 2-8.

Table 2-8 Peptides

\begin{tabular}{|c|c|c|}
\hline Peptide & Sequence & Supplier \\
\hline $\begin{array}{l}\text { H3(1-15)unmod- } \\
\text { FAM }\end{array}$ & ARTKQTARKSTGGKA-K-FAM & $\begin{array}{l}\text { Prof. Dirk Schwarzer, } \\
\text { University of } \\
\text { Tübingen }\end{array}$ \\
\hline $\begin{array}{l}\text { H3(1-15)K9me3- } \\
\text { FAM }\end{array}$ & ARTKQTARK(me3)STGGKA-K-FAM & $\begin{array}{l}\text { Prof. Dirk Schwarzer, } \\
\text { University of } \\
\text { Tübingen }\end{array}$ \\
\hline $\begin{array}{l}\text { FAM-H3(1-15) } \\
\text { unmod }\end{array}$ & FAM-ARTKQTARKSTGGKA-CONH${ }_{2}$ & $\begin{array}{l}\text { Prof. Dirk Schwarzer, } \\
\text { University of } \\
\text { Tübingen }\end{array}$ \\
\hline $\begin{array}{l}\text { FAM-H3(1-15) } \\
\text { K9me3 }\end{array}$ & FAM-ARTKQTARK(me3)STGGKA-CONH ${ }_{2}$ & $\begin{array}{l}\text { Prof. Dirk Schwarzer, } \\
\text { University of } \\
\text { Tübingen }\end{array}$ \\
\hline $\begin{array}{l}\text { H3 unmod 1-20 } \\
\text { biotin }\end{array}$ & ARTKQTARKSTGGKAPRKQLK-biotin & $\begin{array}{l}\text { Prof. Dirk Schwarzer, } \\
\text { University of } \\
\text { Tübingen }\end{array}$ \\
\hline $\begin{array}{l}\text { H3K9me3 1-20 } \\
\text { biotin }\end{array}$ & $\begin{array}{l}\text { ARTKQTARK(me3)STGGKAPRKQLK- } \\
\text { biotin }\end{array}$ & $\begin{array}{l}\text { Prof. Dirk Schwarzer, } \\
\text { University of } \\
\text { Tübingen }\end{array}$ \\
\hline
\end{tabular}




\begin{tabular}{|c|c|c|}
\hline \multicolumn{3}{|l|}{ Cyclic peptides } \\
\hline L1 (biotin) & $\begin{array}{l}\text { yAAIWVKHHYRELVKN-CG-(PEG) } 11-K- \\
\text { biotin }\end{array}$ & $\begin{array}{l}\text { Prof. Hiroaki Suga, } \\
\text { University of Tokyo }\end{array}$ \\
\hline L2 (biotin) & $\begin{array}{l}\text { ySYRIKHHTTKWTKTP-CG-(PEG) } 11-K- \\
\text { biotin }\end{array}$ & $\begin{array}{l}\text { Prof. Hiroaki Suga, } \\
\text { University of Tokyo }\end{array}$ \\
\hline L3 (biotin) & $\begin{array}{l}\text { ySWRIKGHHNRWKQTP-CG-(PEG) }{ }_{11}-\mathrm{K}- \\
\text { biotin }\end{array}$ & $\begin{array}{l}\text { Prof. Hiroaki Suga, } \\
\text { University of Tokyo }\end{array}$ \\
\hline L4 (biotin) & $\begin{array}{l}\text { yRYRVRTHNSRWTVLS-CG-(PEG) }{ }_{11-K-} \\
\text { biotin }\end{array}$ & $\begin{array}{l}\text { Prof. Hiroaki Suga, } \\
\text { University of Tokyo }\end{array}$ \\
\hline L5 (biotin) & $\begin{array}{l}\text { yKSQVRHHPSTKQNNY-CG-(PEG) }{ }_{11-K-} \\
\text { biotin }\end{array}$ & $\begin{array}{l}\text { Prof. Hiroaki Suga, } \\
\text { University of Tokyo }\end{array}$ \\
\hline D1 (biotin) & $\begin{array}{l}\text { yRYWTHTHKSHKTTIP-CG-(PEG) })_{11-K-} \\
\text { biotin }\end{array}$ & $\begin{array}{l}\text { Prof. Hiroaki Suga, } \\
\text { University of Tokyo }\end{array}$ \\
\hline D2 (biotin) & $\begin{array}{l}\text { yTWLDHHDWHRYTRLA-CG-(PEG) } 11-\mathrm{K}- \\
\text { biotin }\end{array}$ & $\begin{array}{l}\text { Prof. Hiroaki Suga, } \\
\text { University of Tokyo }\end{array}$ \\
\hline D3 (biotin) & $\begin{array}{l}\text { yTYKSLIVKNHKRHLS-CG-(PEG) })_{11-K-} \\
\text { biotin }\end{array}$ & $\begin{array}{l}\text { Prof. Hiroaki Suga, } \\
\text { University of Tokyo }\end{array}$ \\
\hline D4 (biotin) & yPYNRILIKRHRVKII-CG-(PEG) ${ }_{11}-\mathrm{K}-$ biotin & $\begin{array}{l}\text { Prof. Hiroaki Suga, } \\
\text { University of Tokyo }\end{array}$ \\
\hline D5 (biotin) & $\begin{array}{l}\text { yPYVLIYVNNHKRPSR-CG-(PEG) })_{11-K-} \\
\text { biotin }\end{array}$ & $\begin{array}{l}\text { Prof. Hiroaki Suga, } \\
\text { University of Tokyo }\end{array}$ \\
\hline L1 (fluorescein) & yAAIWVKHHYRELVKN-CGK-fluorescein & $\begin{array}{l}\text { Prof. Hiroaki Suga, } \\
\text { University of Tokyo }\end{array}$ \\
\hline L2 (fluorescein) & ySYRIKHHTTKWTKTP-CGK- fluorescein & $\begin{array}{l}\text { Prof. Hiroaki Suga, } \\
\text { University of Tokyo }\end{array}$ \\
\hline L3 (fluorescein) & ySWRIKGHHNRWKQTP-CGK- fluorescein & $\begin{array}{l}\text { Prof. Hiroaki Suga, } \\
\text { University of Tokyo }\end{array}$ \\
\hline L4 (fluorescein) & yRYRVRTHNSRWTVLS-CGK-fluorescein & $\begin{array}{l}\text { Prof. Hiroaki Suga, } \\
\text { University of Tokyo }\end{array}$ \\
\hline L5 (fluorescein) & yKSQVRHHPSTKQNNY-CGK- fluorescein & $\begin{array}{l}\text { Prof. Hiroaki Suga, } \\
\text { University of Tokyo }\end{array}$ \\
\hline D1 (fluorescein) & yRYWTHTHKSHKTTIP-CGK- fluorescein & $\begin{array}{l}\text { Prof. Hiroaki Suga, } \\
\text { University of Tokyo }\end{array}$ \\
\hline D2 (fluorescein) & $\begin{array}{l}\text { yTWLDHHDWHRYTRLA-CGK- } \\
\text { fluorescein }\end{array}$ & $\begin{array}{l}\text { Prof. Hiroaki Suga, } \\
\text { University of Tokyo }\end{array}$ \\
\hline D3 (fluorescein) & yTYKSLIVKNHKRHLS-CGK- fluorescein & $\begin{array}{l}\text { Prof. Hiroaki Suga, } \\
\text { University of Tokyo }\end{array}$ \\
\hline D4 (fluorescein) & yPYNRILIKRHRVKII-CGK- fluorescein & $\begin{array}{l}\text { Prof. Hiroaki Suga, } \\
\text { University of Tokyo }\end{array}$ \\
\hline D5 (fluorescein) & yPYVLIYVNNHKRPSR-CGK-fluorescein & $\begin{array}{l}\text { Prof. Hiroaki Suga, } \\
\text { University of Tokyo }\end{array}$ \\
\hline
\end{tabular}

FAM - 5,6-Carboxyfluorescein 


\subsubsection{Oligonucleotides}

All oligonucleotides were obtained from Sigma Life Science (Steinheim).

12mer DNA meth bottom

5'-GTCAG[5MedC]GCATGG-3'

12mer DNA meth top biotin

5'-[btn]-CCATG[5MedC]GCTGAC-3'

12mer DNA bottom

5'-GTCAGCGCATGG-3'

12mer DNA top biotin

5'-[btn]-CCATGCGCTGAC-3'

12mer DNA meth top FAM

5'-[FAM]-CCATG[5MedC]GCTGAC-3'

12mer DNA top FAM

5'-[FAM]-CCATGCGCTGAC-3'

Primers for cloning and mutagenesis are listed in tables 7-1 and 7-2 of the Addendum.

\subsubsection{Plasmids}

Plasmids used for cloning and protein expression in mammalian and bacterial cells are listed in table 2-9.

Table 2-9 Plasmids

\begin{tabular}{|c|c|c|c|c|c|}
\hline Name & Promotor & Tag & Selection & Use & Supplier \\
\hline $\begin{array}{l}\text { pcDNA } \\
\text { 3.1(-)-EE }\end{array}$ & T7 / CMV & $\begin{array}{l}\text { C-terminal } \\
\mathrm{EE}\end{array}$ & Ampicillin & $\begin{array}{l}\text { mammalian } \\
\text { expression } \\
\text { vector }\end{array}$ & $\begin{array}{l}\text { Invitrogen, } \\
\text { Karlsruhe }\end{array}$ \\
\hline $\begin{array}{l}\text { pcDNA } \\
3.1(-) \text {-myc- } \\
\text { His }_{6}\end{array}$ & T7/ CMV & $\begin{array}{l}\text { C-terminal } \\
\text { Myc and } \\
\text { His6 }\end{array}$ & Ampicillin & $\begin{array}{l}\text { mammalian } \\
\text { expression } \\
\text { vector }\end{array}$ & $\begin{array}{l}\text { Invitrogen, } \\
\text { Karlsruhe }\end{array}$ \\
\hline pEGFP-C1 & CMV & $\begin{array}{l}\text { N-terminal } \\
\text { EGFP }\end{array}$ & $\begin{array}{l}\text { Kanamycin, } \\
\text { Neomycin }\end{array}$ & $\begin{array}{l}\text { mammalian } \\
\text { expression } \\
\text { vector }\end{array}$ & $\begin{array}{l}\text { Takara Clontech, } \\
\text { Saint-Germain-en- } \\
\text { Laye (France) }\end{array}$ \\
\hline pet16b & T7 & $\begin{array}{l}\text { N-terminal } \\
\text { His } 10\end{array}$ & Ampicillin & $\begin{array}{l}\text { bacterial } \\
\text { expression } \\
\text { vector }\end{array}$ & $\begin{array}{l}\text { Novagen/Merck, } \\
\text { Mannheim }\end{array}$ \\
\hline $\begin{array}{l}\text { petM13- } \\
\text { His6 }\end{array}$ & T7 & $\begin{array}{l}\text { C-terminal } \\
\text { His } 6\end{array}$ & Kanamycin & $\begin{array}{l}\text { bacterial } \\
\text { expression } \\
\text { vector }\end{array}$ & $\begin{array}{l}\text { EMBL; His6 was } \\
\text { introduced by K. } \\
\text { Gelato, MPI } \\
\text { Göttingen }\end{array}$ \\
\hline $\begin{array}{l}\text { pmCherry- } \\
\text { C1 }\end{array}$ & CMV & $\begin{array}{l}\text { N-terminal } \\
\text { mCherry }\end{array}$ & $\begin{array}{l}\text { Kanamycin, } \\
\text { Neomycin }\end{array}$ & $\begin{array}{l}\text { mammalian } \\
\text { expression } \\
\text { vector }\end{array}$ & $\begin{array}{l}\text { Takara Clontech, } \\
\text { Saint-Germain-en- } \\
\text { Laye (France) }\end{array}$ \\
\hline
\end{tabular}




\subsubsection{Bacterial strains}

Bacterial strains used in this work are listed in table 2-10.

Table 2-10 Bacterial strains

\begin{tabular}{|c|c|c|}
\hline Strain & Genotype & Supplier \\
\hline $\begin{array}{l}\text { BL21-CodonPlus } \\
\text { (DE3)-RIL }\end{array}$ & $\begin{array}{l}\text { E. coli B F-ompT hsdS(rB- mB-) dcm+ } \\
\text { Tetr gal } l \text { (DE3) endA The [argU ileY } \\
\text { leuW } \text { Cam }^{\mathrm{R}} \text { ] }\end{array}$ & Stratagene, La Jolla (USA) \\
\hline Rosetta (DE3) & $\begin{array}{l}\text { F. ompT } h s d S_{\mathrm{B}}\left(\mathrm{r}_{\mathrm{B}}^{-} \mathrm{m}_{\mathrm{B}}^{-}\right) \text {gal dcm (DE3) } \\
\text { pRARE }\left(\mathrm{Cam}^{\mathrm{R}}\right)\end{array}$ & $\begin{array}{l}\text { Novagen, Merck, } \\
\text { Mannheim }\end{array}$ \\
\hline $\mathrm{DH} 5 \alpha$ & $\begin{array}{l}\text { E. coli } \mathrm{F}-\mathrm{f} 80 \text { lacZDM15 D(lacZYA-argF) } \\
\mathrm{U} 169 \text { deoR recA1 endA1 hsdR17(rk', } \\
\text { mk }^{+} \text {) phoAsupE44 l-thi-1 gyrA96 relA1 }\end{array}$ & Invitrogen, Karlsruhe \\
\hline NovaBlue & $\begin{array}{l}\text { endA1 hsdR17 }\left(\mathrm{r}_{\mathrm{K} 12}^{-} \mathrm{m}_{\mathrm{K} 12}{ }^{+}\right) \text {supE } 44 \text { thi-1 } \\
\text { recA1 gyrA96 relA1 lac } \mathrm{F}^{\prime}\left[\text { pro } A+B^{+}\right. \\
\left.\text {lacl }^{q} \mathrm{Z} \Delta M 15:: \operatorname{Tn} 10\right]\left(\mathrm{Tet}^{\mathrm{R}}\right)\end{array}$ & $\begin{array}{l}\text { Novagen, Merck, } \\
\text { Mannheim }\end{array}$ \\
\hline
\end{tabular}

\subsubsection{Mammalian cell lines}

Mammalian cell lines used in this work are listed in Table 2-11.

Table 2-11 Mammalian cell lines

\begin{tabular}{|c|c|c|c|}
\hline Name & Organism & Morphology/Tissue & Disease/Source \\
\hline HeLa & Homo sapiens, human & epithelial/ cervix & adenocarcinoma; ATCC \\
\hline $\mathrm{U}-2$ OS & Homo sapiens, human & epithelial/ bone & osteosarcoma, ATCC \\
\hline NIH/3T3 & Mus musculus, mouse & embryonic fibroblast & $\begin{array}{l}\text { spontaneous } \\
\text { immortalization, ATCC }\end{array}$ \\
\hline MCF7 & Homo sapiens, human & $\begin{array}{l}\text { epithelial/ } \\
\text { mammary gland }\end{array}$ & $\begin{array}{l}\text { metastatic pleural } \\
\text { effusion, ATCC }\end{array}$ \\
\hline IMR90 & Homo sapiens, human & myofibroblast/ lung & finite lifespan, ATCC \\
\hline iMEF JCR & Mus musculus, mouse & embryonic fibroblast & $\begin{array}{l}\text { immortalized; gift from } \\
\text { Prof. Judd Rice, } \\
\text { University of Southern } \\
\text { California, Los Angeles }\end{array}$ \\
\hline iMEF W9 & Mus musculus, mouse & embryonic fibroblast & $\begin{array}{l}\text { immortalized; gift from } \\
\text { Prof. Thomas Jenuwein, } \\
\text { MPI, Freiburg }\end{array}$ \\
\hline
\end{tabular}




\subsection{Molecular biology methods}

\subsubsection{Analysis of nucleic acids}

\subsubsection{Spectrophotometric determination of the concentration of nucleic acids}

The absorbance (A) of nucleic acid solutions at $260 \mathrm{~nm}$ was determined in relation to an adequate buffer control using the Spectrophotometer NanoDrop ND-1000. The concentration was calculated using the average extinction coefficient of double stranded DNA $0,02 \mu \mathrm{g} / \mathrm{ml}^{-1} \mathrm{~cm}^{-1}$, which results in the following equation.

$\mathrm{A}_{260 \mathrm{~nm}}=1 \bumpeq 50 \mu \mathrm{g} / \mathrm{ml}$ double stranded DNA

\subsubsection{Agarose gel electrophoresis}

Nucleic acid solutions were mixed with 10x DNA loading dye in a 1:10 ratio and loaded on the polymerized agarose gel. The size of different DNA fragments was analyzed using $0.5 \%$ to $2 \%$ agarose gels in $1 \mathrm{x}$ TBE Buffer with $0.1 \mu \mathrm{g} / \mathrm{ml}$ ethidium bromide. Samples were run along with a size standard (1 kb Plus Ladder) on agarose gel for $1 \mathrm{~h}$ at 100-120 V using the Sub-Cell-GT electrophoresis system. The DNA was than imaged using a UV transilluminator and gel documentation system ChemiDoc MP.

\subsubsection{Cloning and mutagenesis procedures}

\subsubsection{Polymerase chain reaction (PCR)}

Desired cDNA sequences were amplified by polymerase chain reaction following standard protocols (Mullis et al., 1986), polymerase supplier's protocols. Specific primers (see Addendum Table 7-1) were designed using Lasergene 11. These contained restriction sites suitable for the subsequent cloning procedure. Standard reactions were set up as listed in table 2-12 in $50 \mu 1$ total reaction volume. 


Table 2-12 PCR standard reaction set up
\begin{tabular}{|rl|rc|}
\hline PfuUltra II Fusion HS DNA Polymerase & Q5 High-Fidelity DNA Polymerase \\
\hline 10x PfuUltra II reaction buffer & $1 \mathrm{x}$ & $5 \mathrm{x}$ Q5 reaction buffer & $1 \mathrm{x}$ \\
$\mathrm{dNTP}$ mix & $0.25 \mathrm{mM}$ & dNTP mix & $0.2 \mathrm{mM}$ \\
forward primer & $0.2 \mu \mathrm{M}$ & forward primer & $0.5 \mu \mathrm{M}$ \\
reverse primer & $0.2 \mu \mathrm{M}$ & reverse primer & $0.5 \mu \mathrm{M}$ \\
cDNA template & $50-100 \mathrm{ng}$ & cDNA template & $50-100 \mathrm{ng}$ \\
& & $5 x$ Q5 High GC enhancer & $1 \mathrm{x}$ \\
polymerase & $1 \mathrm{U}$ & polymerase & $1 \mathrm{U}$ \\
\hline
\end{tabular}

The final reaction mix was incubated in a PCR thermocycler epgradient $S$ using the following conditions.

PfuUltra II Fusion HS DNA Polymerase

\begin{tabular}{|cc|}
\hline $\begin{array}{c}\text { temperature } \\
95^{\circ} \mathrm{C}\end{array}$ & \multicolumn{1}{c}{ time } \\
\hline $95^{\circ} \mathrm{C}$ & $30 \mathrm{sec}$ \\
$55-65^{\circ} \mathrm{C}$ & $30 \mathrm{sec}$ \\
$72{ }^{\circ} \mathrm{C}$ & $15 \mathrm{sec} / \mathrm{kb} \mathrm{DNA}$ \\
\hline $72{ }^{\circ} \mathrm{C}$ & $5 \mathrm{~min}$ \\
$10^{\circ} \mathrm{C}$ & $\infty$ \\
\hline
\end{tabular}

Q5 High-Fidelity DNA Polymerase

\begin{tabular}{|cc|}
$\begin{array}{c}\text { temperature } \\
98{ }^{\circ} \mathrm{C}\end{array}$ & time \\
\hline $98^{\circ} \mathrm{C}$ & $10 \mathrm{sec}$ \\
$50-72{ }^{\circ} \mathrm{C}$ & $10 \mathrm{sec}$ \\
$72{ }^{\circ} \mathrm{C}$ & $20-30 \mathrm{sec} / \mathrm{kb} \mathrm{DNA}$ \\
\hline $72{ }^{\circ} \mathrm{C}$ & $2 \mathrm{~min}$ \\
$10^{\circ} \mathrm{C}$ & $\infty$ \\
\hline
\end{tabular}

The PCR amplified DNA fragments were purified using the NucleoSpin Gel and PCR clean up kit for subsequent restriction enzyme digestion according to the manufacturer's 
protocol. Yield and purity of the PCR products were checked by agarose gel electrophoresis.

\subsubsection{Restriction enzyme digest and ligation reaction}

Target vector DNA as well as the PCR-amplified insert DNA were digested with the matching restriction endonucleases (New England Biolabs) according to the manufacturer's instructions. The reaction conditions were optimized using the New England Biolabs double digest finder online tool (https://www.neb.com/tools-andresources/interactive-tools/double-digest-finder). The digested DNA fragments were separated according to their size using agarose gel electrophoresis. The relevant DNA fragments were subsequently extracted from the gel using the NucleoSpin Gel and PCR clean up kit. The purified target vector was dephosphorylated using Antarctic phosphatase following the manufacturer's protocol. For ligation reactions, $50 \mathrm{ng}$ of digested target vector were mixed with 4 to 8 -fold molar excess of the purified insert DNA. The mixture was incubated in a final volume of $20 \mu \mathrm{l} 1 \mathrm{x}$ T4 DNA ligase buffer containing $1 \mu 1 \mathrm{~T} 4$ DNA ligase. For fast ligation, PEG6000 was added to a final concentration of $3 \%[\mathrm{w} / \mathrm{v}]$. Ligation reactions were incubated at $16{ }^{\circ} \mathrm{Co} / \mathrm{n}$ and transformed the next day into NovaBlue chemically competent bacteria.

\subsubsection{Site directed mutagenesis}

Site-directed mutagenesis was performed using specific overlapping primers (see Addendum table 7-3). These carried the appropriate mutation flanked both upstream and downstream by 10 to 18 nucleotides of the native vector sequence (Kunkel, 1985). The following PCR reaction was performed with native Pfu DNA polymerase in a final volume of $50 \mu 1$.

$\begin{array}{rl}\text { 10x Pfu Buffer } & 1 \mathrm{x} \\ \text { MgSO }_{4} & 3 \mathrm{mM} \\ \text { dNTP mix } & 0.2 \mathrm{mM} \\ \text { forward primer } & 0.2 \mu \mathrm{M} \\ \text { reverse primer } & 0.2 \mu \mathrm{M} \\ \text { DNA template } & 200 \mathrm{ng} \\ \text { polymerase } & 2.5 \mathrm{U} / \mu 1\end{array}$

The final reaction mix was incubated in a PCR thermocycler epgradient $S$ using the following conditions. 


\begin{tabular}{|cc|}
\hline $\begin{array}{c}\text { temperature } \\
95^{\circ} \mathrm{C}\end{array}$ & $\begin{array}{c}\text { time } \\
3 \mathrm{~min}\end{array}$ \\
\hline $95^{\circ} \mathrm{C}$ & $30 \mathrm{sec}$ \\
$55-65^{\circ} \mathrm{C}$ & $45 \mathrm{sec}$ \\
$72^{\circ} \mathrm{C}$ & $2 \mathrm{~min} / \mathrm{kb} \mathrm{DNA}$ \\
\hline $72^{\circ} \mathrm{C}$ & $20 \mathrm{~min}$ \\
$10^{\circ} \mathrm{C}$ & $\infty$ \\
\hline
\end{tabular}

The PCR-amplified DNA fragments were digested with DpnI methylation sensitive nuclease for $1 \mathrm{~h}$ at $37^{\circ} \mathrm{C}$ to remove the template plasmid DNA. The final reaction mix was subsequently transformed into NovaBlue chemically competent bacteria.

\subsubsection{Preparation and transformation of chemically competent bacteria}

Competent bacteria were prepared using the calcium chloride $\left(\mathrm{CaCl}_{2}\right)$ method (Dagert and Ehrlich, 1979; Hanahan, 1983; Sambrook and Russell, 2001) and frozen at $-80{ }^{\circ} \mathrm{C}$ for storage. Chemically competent bacteria were transformed by heat shock. For transformation, a 50-100 $\mu 1$ aliquot of competent bacteria was mixed with 10-50 ng of plasmid DNA, 5-10 $\mu$ l of ligation mix or $5 \mu 1$ mutagenesis reaction. Reactions were incubated on ice for $20 \mathrm{~min}$. The bacteria were subsequently heat shocked at $42{ }^{\circ} \mathrm{C}$ for 30-45 sec in a thermomixer and cooled for $2 \mathrm{~min}$ on ice. The heat shocked bacteria were recovered by addition of $450 \mu \mathrm{l}$ SOC medium and were then incubated in a thermomixer at $37{ }^{\circ} \mathrm{C}$ at $850 \mathrm{rpm}$. After $1 \mathrm{~h}$ cells were plated on LB agar supplemented with antibiotic for selection ( $100 \mu \mathrm{g} / \mathrm{ml}$ ampicillin, $50 \mu \mathrm{g} / \mathrm{ml}$ kanamycin) and plates were incubated $\mathrm{o} / \mathrm{n}$ at $37^{\circ} \mathrm{C}$.

\subsubsection{Propagation and preparation of plasmid DNA}

A single colony was picked from the transformation plate for propagation and grown in 5 $\mathrm{ml}$ LB medium with antibiotic at $37^{\circ} \mathrm{C}$ for $16 \mathrm{~h}$. Bacteria were harvested by centrifugation at 3,200x $\mathrm{g}$ and the LB medium was decanted. The plasmid DNA was purified by alkaline lysis of the bacterial pellet (Birnboim and Doly, 1979) and subsequent DNA isolation with a silica membrane spin column. The isolation was performed with the Nucleospin Plasmid purification kit according to the manufacturer's protocol. Purified plasmid DNA was analyzed by restriction enzyme digest and subsequent gel electrophoresis. Plasmid DNA was sent to SeqLab laboratories (Göttingen) for sequencing. Sequencing primers are listed in the Addendum table 7-3. The sequenced and validated plasmid DNA of the desired plasmid were stored at $-20^{\circ} \mathrm{C}$ in $5 \mathrm{mM}$ Tris/ $\mathrm{HCl} \mathrm{pH} \mathrm{8.5.}$ 
For large-scale preparation of plasmid DNA a single colony was picked from the transformation plate for propagation and grown in $200 \mathrm{ml}$ of LB medium containing antibiotics. DNA purification was carried out using the NucleoBond Xtra Midi Plus kit.

\subsubsection{Oligonucleotide annealing}

The lyophilized oligonucleotides listed under 2.1.11 were dissolved in DNA hybridization buffer and complementary strands were mixed for annealing of double stranded DNA. The mix was incubated at $95^{\circ} \mathrm{C}$ for $15 \mathrm{~min}$ and than slowly cooled down to RT.

\subsection{Protein biochemistry}

\subsubsection{Analysis of proteins}

\subsubsection{Spectrophotometric determination of protein concentration}

The concentration of protein solutions was determined using the spectrophotometer NanoDrop ND-1000. For this, absorbance at $280 \mathrm{~nm}$ was measured. Concentrations were determined using the molecular weights in combination with the theoretical molar extinction coefficients calculated on the basis of the amino acid sequence with the aid of the ProtParam online tool (http:// web.expasy.org/protparam/ (Gasteiger et al., 2003)). Final concentrations were calculated according to the following formula derived from the Lambert-Beer equation:

$$
c=\frac{A(\lambda 280)}{\varepsilon \times d}
$$

with A ( $\lambda$ 280) being the absorbance of the solution at $280 \mathrm{~nm}, \varepsilon$ representing the theoretical molar extinction coefficient, $\mathrm{d}$ being the path length of the photometer, resulting in $\mathrm{c}$ the molar protein concentration of the solution. The molar concentration was multiplied with the molecular weight $[\mathrm{g} / \mathrm{mol}]$ to calculate the $[\mathrm{w} / \mathrm{v}]$ concentration.

Table 2-13 Molecular weight and molar extinction coefficient of proteins used in this study

\begin{tabular}{ccc}
\hline Protein & $\begin{array}{c}\text { Molecular weight } \\
{[\mathbf{g} / \mathbf{m o l}]}\end{array}$ & $\begin{array}{c}\text { Molar extinction } \\
\text { coefficient }\left[\mathbf{M}{ }^{-1} \mathbf{c m}^{-1}\right]\end{array}$ \\
\hline$\underline{\text { UHRF1 }}$ & & \\
FL WT His 6 & 90,992 & 118,855 \\
FL WT His 10 & 91,555 & 118,855 \\
\hline
\end{tabular}




\begin{tabular}{lcc}
\hline TTD His6 & 22,247 & 35,535 \\
PHD His6 & 9,920 & 9,105 \\
TTD-PHD His 6 & 32,294 & 44,640 \\
PBR-Ring His6 & 22,661 & 20,440 \\
\hline$\underline{\text { NP95 }} \quad$ & 20,465 & \\
TTD His6 & 22,954 & 35,410 \\
3xFlag TTD His6 & 21,171 & 39,880 \\
PBR-Ring His6 & 22,356 & 12,950 \\
Myc PBR-Ring His6 & & 12,950 \\
NP95 I & 89,482 & 113,220 \\
FL WT His6 & 30,956 & 43,890 \\
TTD-PHD His6 & 30,772 & 40,910 \\
TTD-PHD YY mt His6 & & \\
NP95 II & 88,397 & 113,220 \\
FL WT His6 & 30,208 & 43,890 \\
TTD-PHD His6 & & \\
\hline
\end{tabular}

\subsubsection{Polyacrylamide gel electrophoresis (SDS-PAGE)}

Protein solutions were mixed with the adequate amount of 6x SDS loading buffer and boiled at $95{ }^{\circ} \mathrm{C}$ for $10 \mathrm{~min}$ to denature and prepare the sample for discontinuous polyacrylamide gel electrophoresis in presence of sodium dodecyl sulfate (SDS) (Gallagher, 2007; Laemmli, 1970). Tris glycine gels were used for protein separation with $4 \%$ stacking gel and 10-20\% resolving gel (Chapter 2.1.4). SDS-PAGE gels were cast and run using the Mini-Protean gel casting system. Protein samples were separated at 30-50 mA (300 V max) in 1x SDS running buffer along side with a reference SeeBlue Plus2 Prestained protein marker.

\subsubsection{Western blot analysis}

After SDS-PAGE electrophoresis, the gel, a nitrocellulose membrane and Whatman chromatography paper were equilibrated in transfer buffer $(20 \%$ [v/v] methanol, 1x SDS running buffer) and arranged in a pile. The protein transfer for immunoblotting (Burnette, 1981; Towbin et al., 1979) was performed using the MiniTrans Blot system. The equilibrated stack was packed with a Mini Gel holder cassette and placed with the core unit in the buffer tank containing transfer buffer. The proteins were then transferred to 
the nitrocellulose membrane at constant voltage setting $100 \mathrm{~V}$ for $60-90 \mathrm{~min}$ at $4{ }^{\circ} \mathrm{C}$. To check the transfer of proteins, the nitrocellulose membrane was incubated with Ponceau stain for 2 min at RT and then destained with water. Subsequently, the membrane was blocked with PBST (1x PBS, 0.1\% [v/v] Tween20) containing 5\% [w/v] BSA or milk powder for 30-60 min at RT. The membrane was then incubated with primary antibody solution (Table 2-7) containing PBST, $0.02 \%$ sodium azide and $2.5 \%$ [w/v] BSA or milk powder for $1 \mathrm{~h}$ at $\mathrm{RT}$ or $\mathrm{o} / \mathrm{n}$ at $4{ }^{\circ} \mathrm{C}$. After primary antibody incubation, the blot was washed three times with PBST and the according HRP-coupled secondary antibody solution (Table 2-7) in PBST was added. After $1 \mathrm{~h}$ incubation at RT, the membrane was again washed with PBST three times. The immunostaining was than visualized by an HRP-dependent chemiluminescence reaction using ECL or ECL Plus Western Blotting Detection Reagent according to the manufacturer's instructions. The luminescence was documented using the gel documentation system ChemiDoc MP.

\subsubsection{Coomassie staining of polyacrylamide gels}

After running, SDS-PAGE gels were stained using Coomassie Blue staining solution to visualize proteins (Vesterberg et al., 1977). Gels were incubated with the stain for 20-30 min while rocking and subsequently destained with destaining solution for $1 \mathrm{~h}$ or water $\mathrm{o} / \mathrm{n}$ at $\mathrm{RT}$.

\subsubsection{Expression and purification of proteins}

\subsubsection{Bacterial expression}

Chemically competent BL21 (DE3) codon plus RIL were transformed with a bacterial expression vector carrying the coding sequence of the required protein. A single colony of this transformation was then used to inoculate a pre-culture of $200 \mathrm{ml}$ 2xYT medium with $0.4 \%$ glucose and antibiotic. The pre-culture was incubated for $16 \mathrm{~h}$ at $37^{\circ} \mathrm{C}, 125 \mathrm{rpm}$ and was subsequently diluted $1: 40$ in $3 \mathrm{~L}$ of pre-warmed 2 XYT medium containing $0.4 \%$ glucose and antibiotic. Bacterial cultures were grown at $37{ }^{\circ} \mathrm{C}, 125 \mathrm{rpm}$ to $\mathrm{OD}_{600}$ of 0.5 . Protein expression was induced by adding $0.5 \mathrm{mM}$ IPTG at $25^{\circ} \mathrm{C}$ for $3-4 \mathrm{~h}$ (Gelato et al., 2014; Sambrook and Russell, 2001).

Proteins that were used for cyclic peptide screening experiments (UHRF1 FL WT His10 and UHRF1 FL RKS 649/650/651 AAA mt His10) were transformed into chemically competent E. coli Rosetta and treated as described above. The culture was then diluted 1:50 in the described medium and grown until it reached an $\mathrm{OD}_{600}$ of 0.5 . The protein expression was induced using $0.5 \mathrm{mM}$ IPTG and incubated $\mathrm{o} / \mathrm{n}$ at $18^{\circ} \mathrm{C}$ and $125 \mathrm{rpm}$. 
Subsequently, bacteria were pelleted by centrifugation at 6,200x g for $15 \mathrm{~min}$ at $4{ }^{\circ} \mathrm{C}$ and the bacterial pellets were stored at $-20^{\circ} \mathrm{C}$ until further use.

\subsubsection{Purification by metal ion affinity chromatography}

UHRF1 FL and domains were purified at $\mathrm{pH}$ 8.0, while NP95 FL proteins and domains were purified at $\mathrm{pH}$ 7.5. The bacterial pellets of $3 \mathrm{~L}$ cultures were resuspended in $40 \mathrm{ml}$ lysis buffer. Protease inhibitor cocktail (EDTA-free) was added immediately before the purification to the pre-cooled lysis buffer. The resuspended bacteria were lysed by 3-4 passages at 1,000-1,500 bar through the EmulsiFlex C5 cell disruptor. Bacterial cell debris and insoluble material was removed by centrifugation at 25,000x g for $25 \mathrm{~min}$ at $4{ }^{\circ} \mathrm{C}$. The supernatant was applied to $5 \mathrm{ml}$ HisPur Cobalt resin equilibrated with lysis buffer in a gravity flow column and was loaded onto the column twice. The resin was than washed with 150 to $250 \mathrm{ml}$ of pre-cooled wash buffer and the protein was subsequently eluted in 20-25 ml elution buffer. NP95 PBR-RING was washed with $250 \mathrm{ml}$ of wash buffer containing $1 \mathrm{M} \mathrm{NaCl}$. Eluted fractions were dialyzed $\mathrm{o} / \mathrm{n}$ against dialysis buffer with freshly added DTT using SpectraPor MWCO 3,500 Da dialysis tubing. Purified proteins were checked by SDS-PAGE electrophoresis. Subsequently, the proteins were concentrated using Amicon Ultra centrifugal filter units and stored at $4{ }^{\circ} \mathrm{C}$.

\subsubsection{Biophysical and biochemical binding assays}

\subsubsection{Fluorescence polarization (FP)}

Fluorescence polarization experiments (Tota et al., 1994) were performed using the HIDEX PlateChameleon or TECAN Infinite M1000 Pro plate reader at $4{ }^{\circ} \mathrm{C}$ and RT. The association of fluorescently labeled peptides with purified recombinant protein was measured. The protein solution was adjusted to a starting concentration of 50 or $100 \mu \mathrm{M}$. The solution was then repeatedly diluted 1:1 resulting in a 12 step dilution series. Fluorescent peptide (final concentration $10 \mathrm{nM}$ ) was mixed with each protein dilution in a final reaction volume of $10 \mu \mathrm{l}$ per measurement point and reactions were transferred to a black flat bottom 384-well plate. Binding reactions were incubated on ice for $10 \mathrm{~min}$ and transferred to the plate reader. The fluorophore was excited using linear polarized light at $485 \mathrm{~nm}$ and the intensity of parallel and perpendicular orientated light emitted at $535 \mathrm{~nm}$ was measured. Fluorescence polarization was calculated from the measured intensities using the following equation:

$$
P=\frac{I_{\|}-G * I_{\perp}}{I_{\|}+G * I_{\perp}}
$$


$I_{\|}$is the intensity of the parallel oriented emitted light; $I_{\perp}$ is the intensity of the perpendicular oriented emitted light; $G$ is a device specific factor. The anisotropy values were calculated by:

$$
A=\frac{2 P}{3-P}
$$

Datasets were analyzed using KaleidaGraph and the data points in each dilution series were fit with the equation:

$$
\mathrm{y}=\mathrm{m} 1+(\mathrm{m} 2-\mathrm{m} 1) * \frac{\mathrm{M} 0}{\mathrm{KD}+\mathrm{M} 0}
$$

For: $Y=F(M 0)$

With $\mathrm{m} 1$ and $\mathrm{m} 2$ being the minimum and maximum boundary values, respectively, and $\mathrm{KD}$ the estimated dissociation constant. Each titration series was normalized to a curve where $\mathrm{m} 1(\operatorname{Min})=0$ and $\mathrm{m} 2(\operatorname{Max})=1$, and then averaged with the normalized curves of all replicates with a minimum of three independent measurements.

\subsubsection{Peptide pull down assay with recombinant protein (PPD)}

For peptide pull down assays, $40 \mu 1$ streptavidin paramagnetic bead suspension (MagneSphere) were used per reaction. Beads were washed three times with PBS in lowbinding tubes before incubation in $200 \mu \mathrm{l}$ PBS with $10 \mu \mathrm{g}$ biotin labeled peptide (H3 unmodified, H3K9me3) or equivalent amount water for $2 \mathrm{~h}$ at $4{ }^{\circ} \mathrm{C}$ with rotation. The peptide-loaded or unloaded control beads were washed three times with PBS and equilibrated with PD300 buffer (20 mM HEPES pH 7.9/7.5, $300 \mathrm{mM} \mathrm{KCl,} 0.2 \%$ [v/v] Triton X-100, 20\% [v/v] glycerol). Subsequently, the beads were charged with 5-10 $\mu \mathrm{g}$ recombinant protein and the reaction was adjusted to $500 \mu 1$ with PD300 buffer. Each peptide pull down reaction was then incubated rotating at $4{ }^{\circ} \mathrm{C}$ for $3-5 \mathrm{~h}$. Beads were washed six times with $200 \mu 1$ PD300 buffer and the supernatant carefully and thoroughly removed. The beads were resuspended in $20 \mu \mathrm{l}$ 6x SDS loading buffer and eluted by boiling for 5 minutes.

\subsubsection{Co-immunoprecipitation (Co-IP)}

Co-IP with recombinant UHRF1 domains: For co-immunoprecipitation, $0.22 \mathrm{nmol}$ recombinant UHRF1 PBR-RING were mixed with $1 \mu \mathrm{l} \alpha$-UHRF1 antibody. BSA was added to $5 \%[\mathrm{w} / \mathrm{v}]$ final concentration. The mix was incubated for $1 \mathrm{~h}$ at $4{ }^{\circ} \mathrm{C}$. In parallel, $10 \mu \mathrm{L}$ Dynabeads M280 sheep anti mouse (Invitrogen) were washed once with PBS, twice with IP150 buffer (50 mM Tris- $\mathrm{HCl} \mathrm{pH} 8.0,150 \mathrm{mM} \mathrm{NaCl}, 5 \%$ [v/v] glycerol, 0.05\% [v/v] Triton X-100), and resuspended in IP150 buffer supplemented with 5\% [w/v] BSA. After 
incubation, the $10 \mu \mathrm{l}$ equilibrated beads were added to the UHRF1 PBR-RING/antibody mixture and the reaction was rotated at $4{ }^{\circ} \mathrm{C}$. After $1 \mathrm{~h}$ incubation, $0.22 \mathrm{nmol}$ UHRF1 TTD or TTD-PHD were added to UHRF1 PBR-RING/antibody/bead mix for $3 \mathrm{~h}$. All Co-IP reactions were washed four times, 1 min each with $150 \mu 1$ IP150 buffer and eluted using $20 \mu 1$ 6x SDS loading buffer (Gelato et al., 2014).

Co-IP reactions with $\mathrm{H} 3$ peptide as competitor: biotin-labeled unmodified $\mathrm{H} 3$ or $\mathrm{H} 3 \mathrm{~K} 9 \mathrm{me} 3$ biotin peptides were pre-incubated with UHRF1 TTD for 30-60 min before addition to UHRF1 PBR-RING/antibody/bead mixture. The molar ratio of UHRF1 TTD to H3 peptide was 1:5. All other steps were carried out as described above.

Co-IP reactions with PIPs as competitor: UHRF1 PBR-RING and PIPs were mixed in a molar ratio of $1: 10$ and incubated at $4{ }^{\circ} \mathrm{C}$ o/n, before $\alpha$-UHRF1 antibody was added. For Co-IP with PIP, the detergent was omitted in the IP150 buffer. The subsequent steps outlined above were carried out as described.

Co-IP reactions with cyclic peptide as competitor: UHRF1 PBR-RING and the cyclic peptides were mixed at a molar ratio of $1: 5$ and incubated at $4{ }^{\circ} \mathrm{C} o / n$ before the $\alpha$-UHRF1 antibody was added to the mixture. The subsequent steps were carried out as described above.

Co-IP with recombinant NP95 domains I: For co-immunoprecipitation, $10 \mu 1$ Myc-Tag mouse mAB magnetic bead conjugate were washed once with PBS, twice with IP150 buffer, and resuspended in IP150 buffer supplemented with 5\% [w/v] BSA. The beads were mixed with $0.44 \mathrm{nmol}$ recombinant NP95 myc-PBR-RING and BSA was added to $5 \%$ [w/v] final concentration. After $3 \mathrm{~h}$ incubation at $4{ }^{\circ} \mathrm{C}$ with rotation, the NP95 myc-PBRRING/antibody/bead mix was supplemented with $1.1 \mathrm{nmol}$ of NP95 I or NP95 II TTDPHD. After three more hours of incubation, all Co-IP reactions were washed four times 1 min each with $150 \mu 1$ IP150 buffer and eluted using $20 \mu 1$ 6x SDS loading buffer.

Co-IP with recombinant NP95 domains II: For co-immunoprecipitation, $0.22 \mathrm{nmol}$ recombinant NP95 3xFLAG-TTD were mixed with $1 \mu 1 \alpha$-Flag M2 antibody and BSA was added to $5 \%[\mathrm{w} / \mathrm{v}]$ final concentration. The mix was incubated for $1 \mathrm{~h}$ at $4{ }^{\circ} \mathrm{C}$ with rotation. In parallel, $10 \mu \mathrm{L}$ Dynabeads M280 sheep anti mouse were washed once with PBS and twice with IP150 buffer. The beads were then resuspended in IP150 buffer supplemented with 5\% [w/v] BSA. After Incubation, the $10 \mu \mathrm{l}$ equilibrated beads were added to the NP95 3xFLAG-TTD/antibody mixture and the reaction was rotated at $4{ }^{\circ} \mathrm{C}$. After $1 \mathrm{~h}$, 0.44 nmol NP95 PBR-RING were added to NP95 3xFlag/antibody/bead mix and incubated with rotation for 3 more hours. All Co-IP reactions were washed four times 
with $150 \mu 1$ IP150 buffer and eluted using $20 \mu 1$ 6x SDS loading buffer. Samples were analyzed by SDS-PAGE followed by western blotting, using $\alpha$-His primary antibody.

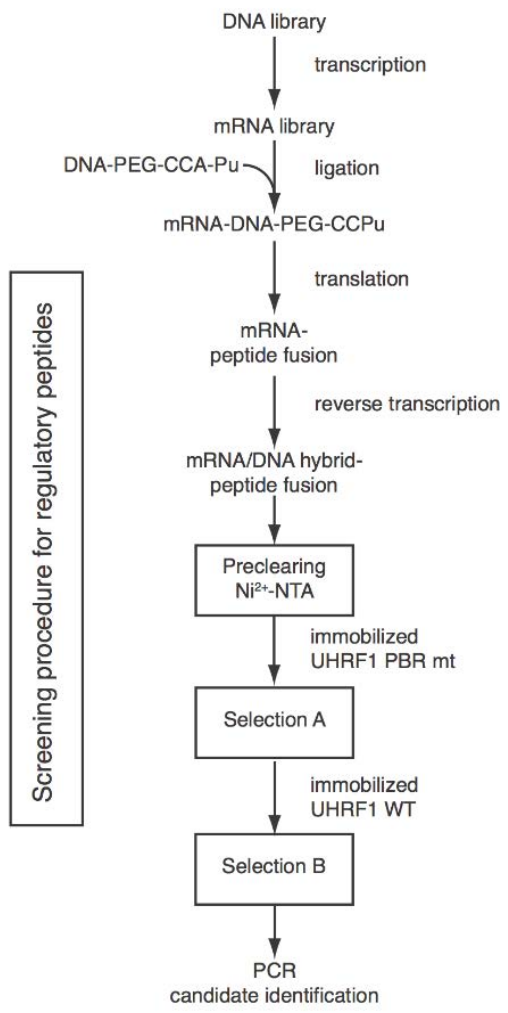

Figure 2-1 Flow scheme of cyclic peptide screening procedure.

\subsubsection{Cyclic peptide screening}

Shiori Umemoto (Laboratory of Hiroaki Suga, University of Tokyo) generated the short random peptides from a DNA library by transcription, fusion to DNA-PEG-CCAPuromycin resistance and subsequent translation (Figure 2-1) (Hayashi et al., 2012). This resulted in a hybrid containing not only the translated peptide but also the associated mRNA. The translated peptide underwent a spontaneous macro-cyclization involving the terminal tyrosine and cysteine residues of the peptides. A reverse transcription step created an mRNA/DNA hybrid that was in the end used for peptide identification using PCR. The affinity selection of the cyclic peptides consisted of three steps. First, preclearing was used to eliminate the unspecific peptides. Second, selection A used full length UHRF1 R649A/K650A/S651A mutant to purge all peptides binding to the protein body but not the PBR. Third, selection B with WT UHRF1 selected the peptides of the resulting pool that were bound by the PBR. Repeating the selection procedure led to conversion of the whole library and enrichment of the final peptide candidates. The remaining identified candidates and their sequences are listed in the materials section (Table 2-8). 


\subsection{Cell biology methods}

\subsubsection{Cell culture, transfection and synchronization}

\subsubsection{Passaging of mammalian cell lines}

Human and murine cell lines were cultured in Dulbecco's modified Eagle's medium (DMEM) containing 1x GlutaMax I, $4.5 \mathrm{~g} / 1$ D-glucose, 1x non-essential amino acids, $1 \mathrm{mM}$ sodium pyruvate and 10\% HyClone BGS. Cells were seeded in $10 \mathrm{ml}$ culture medium in a $100 \mathrm{~mm}$ petri dish and grown at $37{ }^{\circ} \mathrm{C}$ and $5 \% \mathrm{CO}_{2}$ to confluency. For passaging, the culture medium was removed and the cells were washed with DPBS. The cells were detached and separated by incubation with $1 \mathrm{ml}$ of $0.05 \%$ Trypsin/EDTA solution for 2-5 min at $37^{\circ} \mathrm{C}$ and $5 \% \mathrm{CO}_{2}$. Cells were then suspended in $9 \mathrm{ml}$ fresh medium and harvested by centrifugation for $5 \mathrm{~min}$ at $600-800 \times \mathrm{g}$. The medium was decanted; the cells were resuspended and diluted 1:5 to 1:10 in $10 \mathrm{ml}$ of fresh culture medium.

For freezing, cells were harvested as described above and resuspended in TC-Protector cell freezing medium. Aliquots of cells were stored in cryotubes at $-152{ }^{\circ} \mathrm{C}$.

\subsubsection{Transfection of mammalian cells}

Transfection was carried out using the Lipofectamine LTX and Plus Reagent. Before each transfection, mammalian cells were grown in 6-well cell culture dish or on glass cover slips to 50\% confluency. Per well, $150 \mu$ l Opti-MEM were supplemented with $3 \mu 1$ of Lipofectamine LTX. In parallel, vector DNA (see below) and $3 \mu 1$ of Plus Reagent were added to an additional $150 \mu$ l Opti-MEM. Both solutions were mixed thoroughly and pooled. After $5 \mathrm{~min}$ incubation at RT, $250 \mu \mathrm{l}$ of the resulting mixture were added to the cells growth medium. Each transfection were incubated at $37^{\circ} \mathrm{C}$ with $5 \% \mathrm{CO}_{2}$ for $14-16 \mathrm{~h}$.

For localization studies, cells were transfected with mammalian expression vectors pmCherry / pEGFP-C1 carrying the coding sequence of UHRF1, NP95 I, NP95 II FL WT or mt.

For competition experiments, $1.25 \mu \mathrm{g}$ pmCherry UHRF1 FL WT or SRA mt vectors were co-transfected with $2.5 \mu \mathrm{g}$ pEGFP-C1 PBR WT or mt expression vector per 6-well. PI5P 4kinase $\alpha$-EE and PI5P 4-kinase $\beta$-myc in a pcDNA3.1 backbone were a gift from Nullin Divecha (Centre of biological sciences, University of Southampton) (Ndamukong et al., 2010). 
For PI5P 4-kinase $\alpha / \beta$ experiments, $1.25 \mu \mathrm{g}$ of each WT or the mt (PI5P 4-kinase $\alpha$ G131L/Y138F and PI5P 4-kinase $\beta$ D278A) vectors was co-transfected with $2.5 \mu \mathrm{g}$ pmCherry WT UHRF1 plasmid.

Large-scale transfection in $150 \mathrm{~mm}$ petri dishes was carried out using the CalPhos Kit following the manufacturer's protocol. The vector DNA $(45 \mu \mathrm{g})$ was mixed with $198.5 \mu \mathrm{l}$ of $2 \mathrm{M}$ calcium solution and diluted with sterile water up to final volume of $1.6 \mathrm{ml}$. The mix was vortexed while $1.6 \mathrm{ml}$ of 2x HEPES-buffered saline was added gradually. After 10 min incubation, the solution was gently mixed and added to the culture dish. The cells were incubated with the mix o/n at $37^{\circ} \mathrm{C}$ and $5 \% \mathrm{CO}_{2}$.

\subsubsection{Synchronisation of mammalian cells}

Cells were synchronized in M phase of the cell cycle using a thymidine/ nocodazole block (Whitfield et al., 2000). Cells were grown to 30-40\% confluency in described growth medium and then treated with $2 \mathrm{mM}$ thymidine for 20-24 h to arrest the cell cycle in $\mathrm{S}$ phase. The arrest was released by washing the cells with DPBS and culture medium. Subsequently, the cells were covered with fresh growth medium and allowed to progress to $\mathrm{G} 2$ phase. After $3 \mathrm{~h}$ release, $100 \mathrm{ng} / \mathrm{ml}$ nocodazole was added to the culture medium for $12 \mathrm{~h}$ to arrest the progression of the cell cycle in M phase. Thereafter, the cells were released from the block as described and the cell cycle progression was analyzed by flow cytometry.

\subsubsection{Extract preparation}

\subsubsection{Cytosolic and nuclear extract preparation (rapid method)}

Rapid nuclear extract preparation (Osborn et al., 1989) was used for peptide pull down with nuclear extract and expression analysis by SDS-PAGE gel and western blot. The standard protocol for a confluent $150 \mathrm{~mm}$ petri dish was scaled depending on the experimental conditions. Cells were pelleted by centrifugation at 300x $\mathrm{g}$ for $5 \mathrm{~min}$ and the medium was decanted. The pellet was washed with $10 \mathrm{ml}$ PBS once and with $500 \mu 1$ buffer A (10 mM HEPES-NaOH pH 7.9 at $4{ }^{\circ} \mathrm{C}, 1.5 \mathrm{mM} \mathrm{MgCl}_{2}, 10 \mathrm{mM} \mathrm{KCl}, 1 \mathrm{mM}$ DTT and $1 \mathrm{x}$ protease inhibitor cocktail) twice. After centrifugation at $600 \times \mathrm{g}$ at $4{ }^{\circ} \mathrm{C}$, the washed cell pellet was resuspended in $500 \mu \mathrm{l}$ buffer A with $0.2 \%[\mathrm{v} / \mathrm{v}] \mathrm{NP} 40$ and incubated on ice for $10 \mathrm{~min}$ to lyse the cell membranes. The nuclei were pelleted at $600 \mathrm{x} \mathrm{g}$ and the supernatant was saved (cytosolic fraction). The nuclear pellet was resuspended in buffer C (20 mM HEPES-NaOH pH 7.9 at $4{ }^{\circ} \mathrm{C}, 25 \%$ [v/v] glycerol, $420 \mathrm{mM} \mathrm{NaCl}, 0.2 \mathrm{mM}$ EDTA, $1.5 \mathrm{mM}$ $\mathrm{MgCl}_{2}, 1 \mathrm{mM}$ DTT and 1x protease inhibitor cocktail) and the nuclei were lysed using a 
pestle B size homogenizer 20 times every $5 \mathrm{~min}$ for a total of $15 \mathrm{~min}$ on ice. Lysed nuclei were spun at $20,000 \mathrm{x}$ for $10 \mathrm{~min}$ at $4{ }^{\circ} \mathrm{C}$ to pellet insoluble material. The supernatant was removed and diluted with $600 \mu \mathrm{l}$ buffer D (20 mM HEPES-NaOH pH 7.9 at $4{ }^{\circ} \mathrm{C}, 20 \%$ [v/v] glycerol, $50 \mathrm{mM} \mathrm{KCl}, 0.2 \mathrm{mM}$ EDTA). Cytosolic and nuclear extracts were flash frozen in liquid nitrogen and stored at $-80{ }^{\circ} \mathrm{C}$.

\subsubsection{Nuclear extract preparation with MNase}

A standard protocol (O'Loghlen et al., 2012) is described for a confluent $150 \mathrm{~mm}$ petri dish. This was scaled depending on the experimental conditions. Cells were pelleted by centrifugation at $300 \mathrm{x}$ for $5 \mathrm{~min}$ and the medium was decanted. The pellet was washed with $10 \mathrm{ml}$ PBS twice. Nuclei were isolated by $20 \mathrm{~min}$ incubation with $1 \mathrm{ml}$ pre-cooled sucrose buffer (0.32 $\mathrm{M}$ sucrose, $10 \mathrm{mM}$ Tris- $\mathrm{HCl} \mathrm{pH}$ 8.0, $3 \mathrm{mM} \mathrm{CaCl}_{2}, 2 \mathrm{mM} \mathrm{MgOAc}, 0.1$ mM EDTA, 1 mM DTT, 0.5-1\% Triton X-100 and 1x protease inhibitor cocktail EDTA-free) on ice. Nuclei were recovered by centrifugation at 2,000x $\mathrm{g}$ for $5 \mathrm{~min}$ at $4{ }^{\circ} \mathrm{C}$ and washed once with sucrose buffer without detergent. The nuclear pellet was than resuspended in $250 \mu 1$ lysis buffer (20 mM HEPES-KOH pH 7.9, 25\% [v/v] glycerol, $420 \mathrm{mM} \mathrm{KCl,} 1.5 \mathrm{mM}$ $\mathrm{MgCl}_{2}, 0.2 \mathrm{mM}$ EDTA, $1 \mathrm{mM}$ DTT and 1x protease inhibitor cocktail EDTA-free) and subjected to repeated freeze-thaw cycles using liquid nitrogen and a $37^{\circ} \mathrm{C}$ water bath. Insoluble material was pelleted by centrifugation at $2,000 \mathrm{x}$ g at $4{ }^{\circ} \mathrm{C}$. The first supernatant was saved and kept on ice and the pellet was resuspended in MNase buffer $(20 \mathrm{mM}$ HEPES-KOH pH 7.9, $100 \mathrm{mM} \mathrm{KCl}, 1 \mathrm{mM} \mathrm{CaCl}_{2}, 1.5 \mathrm{mM} \mathrm{MgCl}$, $1 \mathrm{mM}$ DTTand 1x protease inhibitor cocktail EDTA-free). The chromatin fraction was solubilized by digestion with 100-200 $\mathrm{U}$ of MNase at $37^{\circ} \mathrm{C}$ for 15-30 min depending on the cell line. The reaction was subsequently quenched by addition of $4 \mathrm{mM}$ EDTA. After 5 min incubation on ice, the first supernatant and the MNase digest were spun at 16,000x $\mathrm{g}$ for $15 \mathrm{~min}$. The two supernatants were pooled and the $\mathrm{KCl}$ concentration was adjusted to $150 \mathrm{mM}$ final with a salt-free lysis buffer.

\subsubsection{Staining procedures of mammalian cells}

\subsubsection{Immunofluorescence}

Cells were grown on glass cover slips until $80 \%$ confluency was reached. After gently washing the cells twice with PBS, these were fixed with $3.7 \%[\mathrm{v} / \mathrm{v}]$ formaldehyde/PBS solution for $10 \mathrm{~min}$. Formaldehyde was removed by thoroughly washing cells with PBS. Fixed cells were permeabilized by incubation with $0.5 \%$ Triton-X-100/PBS for 5 min and then blocked for 30 min in blocking buffer (2.5\% BSA/PBS/ 0.05\% Tween-20). Cells were 
stained with primary antibody (Table 2-7) at $4{ }^{\circ} \mathrm{C}$ for 3-5 h. Subsequently, cells were washed three times for 5 min each with wash buffer (1x PBS/0.05\% Tween-20) and treated with fluorescently labeled secondary antibody (Table 2-7). The cells were carefully washed with wash buffer and PBS. Finally, nuclear DNA was stained 3-5 min with DAPI stain (100 ng/ml DAPI) in wash buffer. Cells were rinsed once with PBS and mounted with vectashield. For experiments with fluorescently labeled proteins, slides were directly stained with DAPI after fixation and mounted in a drop of vectashield.

\subsubsection{Nuclear counterstaining with propidium iodide (PI) staining}

Mammalian cells were synchronized as described. For propidium iodide (PI) staining, cells were sedimented by centrifugation at $800 \mathrm{x} g$ for $5 \mathrm{~min}$ at RT and gently washed with a generous amount of PBS. After PBS wash, cells were detached with a cell scraper and suspended in $100 \mu \mathrm{l}$ PBS. The carefully suspended cells were fixed with $5 \mathrm{ml}$ of ice-cold $70 \%$ ethanol. Fixed cells were stored at $4{ }^{\circ} \mathrm{C}$ for up to four weeks. For PI staining, cells were pelleted by centrifugation at $500 \mathrm{x} \mathrm{g}$ for $5 \mathrm{~min}$ at $4^{\circ} \mathrm{C}$ and the ethanol was removed. Cells were washed twice with PBS/ 0.1\% [v/v] NP40 and were resuspended in staining solution (100 $\mu \mathrm{g} / \mathrm{ml}$ PI, 0.6\% NP40, $1 \mathrm{mg} / \mathrm{ml}$ RNase A in PBS) and incubated for $45 \mathrm{~min}$ on ice in the dark. After incubation, staining solution was diluted with $400 \mu 1$ PBS and the cells were analyzed using a BD FACSCanto II flow cytometer.

\subsubsection{Nuclear counterstaining with Hoechst 33342 staining}

NIH3T3 cells were grown to 70-80\% confluency. $5 \mu \mathrm{g} / \mathrm{ml}$ of Hoechst $33342(1 \mathrm{mg} / \mathrm{ml}$ stock in DMSO) were added to the culture medium and the cells were stained for 45-60 min at $37{ }^{\circ} \mathrm{C}$ with $5 \% \mathrm{CO}_{2}$ (Parrilla et al., 2004). Subsequently, cells were washed twice with DPBS and detached from the culture dish using 0.05\% of Trypsin/EDTA for 2 min. Cells were resuspended in growth medium and pelleted by centrifugation at 800x $\mathrm{g}$ for 5 min. The pellet was washed with DPBS twice and cells were resuspended in DPBS for flow cytometric analysis. 


\subsubsection{Analysis of mammalian cells}

\subsubsection{Fluorescence microscopy}

Immunofluorescence samples were imaged using the Leica TCS SP5 confocal laserscanning microscope equipped with a Leica HCX PL APO lambda blue 63×/1.40-0.60 OIL UV objective lens. DAPI fluorescence was excited by a $405 \mathrm{~nm}$ laser (UV diode), EGFP as well as Alexa 488 were excited with the $488 \mathrm{~nm}$ argon laser and mCherry as well as Alexa546 were excited using the $561 \mathrm{~nm}$ CW-DPSS laser. Image section and channel brightness were adjusted using the Leica Application Suite Advanced Fluorescence (LAS AF). Samples were scanned supported by photo multiplier tubes (PMT) and imaged with a monochrome CCD camera. The pictures were saved at 1024x1024 pixel resolution. Subsequently, the pictures were analyzed and processed using Adobe Photoshop software.

A Zeiss Axiovert 40CFL microscope was used for cell counting and transfection control. The HBO50/AC mercury short-arc lamp (OSRAM) and a beam splitter were used to excite the fluorescent dyes and labels.

\subsubsection{Flow cytometric analysis}

Flow cytometric analysis was carried out using the BD FACSCanto II, which includes three fixed-wavelength lasers for excitation. The collection optics is able to direct fluorescence as well as the light scatter to the according detectors. For flow cytometric analysis, the BD FACSDiva soft ware was used to gate cells depending on forward and side scatter to exclude dead and apoptotic cells from the analysis. Propidium iodide and EGFP fluorescence was excited with a $488 \mathrm{~nm}$ blue laser and Hoechst 33342 fluorescence was excited with the $405 \mathrm{~nm}$ violet laser. In the next analysis step, cells were gated for EGFP fluorescence and then for the nuclear counterstain Hoechst 33342. Alternatively, cells were only gated for PI fluorescence. Cells without staining or fluorescence were excluded from analysis by plotting the forward scatter against the fluorescence intensities. Depending on the intensity and area of the plotted nuclear staining signal, gates were set to only include single cells in the analysis. Only these cells were analyzed for their cell cycle stage using the nuclear stain area histogram. Location and shape of histogram peaks was adjusted manually to fit the optimal histogram shape with the G2/M peak having twice the value of the G1 peak. Subsequently the BD FACSDiva software calculated the percentage of cells in the defined cell cycle stages. 


\section{Results}

\subsection{UHRF1 localization in cells is dependent on linker regions}

\subsubsection{Heterogeneous nuclear localization of UHRF1}

UHRF1 is crucial for the recruitment of chromatin modifying enzymes to differentially modified loci. Thus, the accurate localization of the protein in the nucleus during the cell cycle and differentiation is of great importance (Chapter 1.4). Previous reports showed that inter-domain linker regions regulate UHRF1 interaction with H3K9me3 in vitro. I was particularly interested in the co-localization of UHRF1 with the H3K9me3 modification in cells.

In collaboration with Kyoko Hamada (Laboratory of Wolfgang Fischle, MPI, Göttingen), I investigated the general cellular distribution of human UHRF1 WT protein. We examined the distribution of endogenous and overexpressed UHRF1 WT protein in different mammalian cell lines (NIH3T3, IMR90, MCF7 and U2OS) by immunofluorescence and determined the co-localization of the protein with the H3K9me3 histone modification. In human cell lines we detected the endogenous protein and the modification by indirect immunofluorescence. The analysis with fluorescence microscopy showed that in the analyzed human cell lines, U2OS, MCF7, and IMR90, the distribution pattern of UHRF1 was generally dispersed and only few cells showed strong co-localization of UHRF1 and H3K9me3 (Figure 3-1A).

In murine NIH3T3 cells we transiently expressed mCherry-tagged UHRF1 to evaluate its co-localization with the $\mathrm{H} 3 \mathrm{~K} 9 \mathrm{me} 3$ modification in the cell nucleus. In agreement with previous studies, the majority of NIH3T3 cells showed clear co-localization of transiently expressed mCherry-UHRF1 with H3K9me3. The protein and the histone modification were mostly localized to pericentromeric heterochromatin (Figure 3-1B) (Karagianni et al., 2008) (Papait et al., 2008). 
A
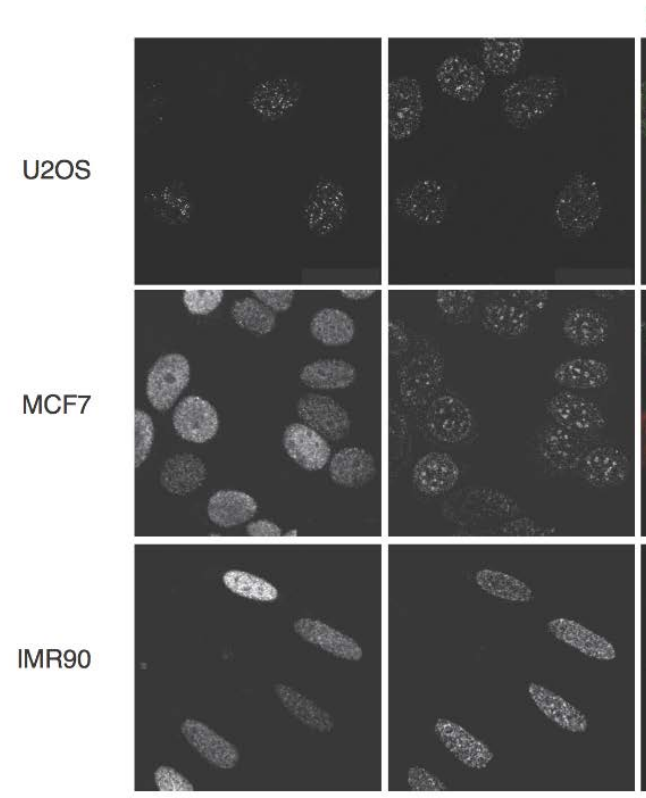

B

NIH3T3

H3K9me3
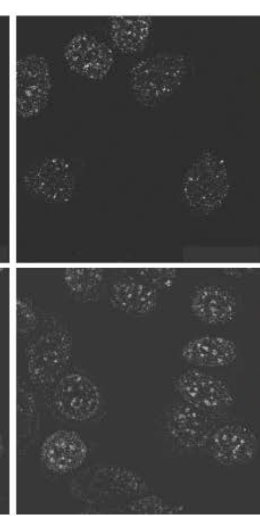

IMR90

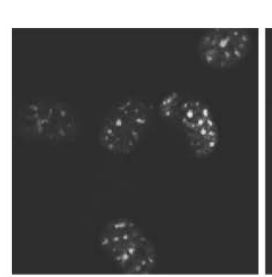

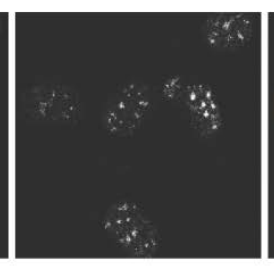

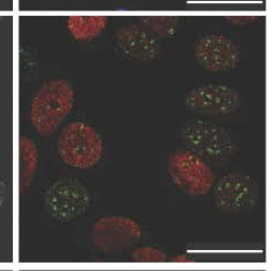

merge

(UHRF1, H3K9me3)
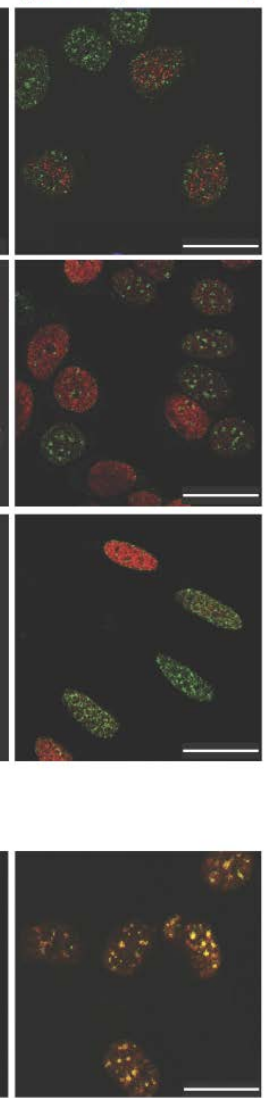

Figure 3-1 UHRF1 nuclear localization in different cell lines

(A) Co-localization of UHRF1 and H3K9me3 modification were analyzed in U2OS, MCF7 and IMR90 cells using specific antibodies. Microscopy images of separate and merged fluorescence channels (UHRF1-red, H3K9me3green) are shown. (B) mCherrytagged UHRF1 was transiently expressed in NIH3T3 cells. H3K9me3 was detected using specific antibodies. Microscopy images of separate and merged fluorescence channels (UHRF1red, H3K9me3-green) are shown. Scale bar represents $25 \mu \mathrm{m}$. Experiments were done in collaboration with Kyoko Hamada (Laboratory of Wolfgang Fischle, MPI, Göttingen).

\subsubsection{The TTD domain is responsible for the localization of UHRF1 to pericentromers}

UHRF1 comprises several domains that can specifically target the protein to heterochromatic regions (Bostick et al., 2007) (Rajakumara et al., 2011) (Liu et al., 2013) (Rothbart et al., 2013). While the exact regulation of UHRF1 sub-nuclear localization and chromatin association is unclear, the protein might rely on different domains for target binding. It was reported that the TTD as well as the SRA domain of UHRF1 contribute to the accurate localization of the protein to pericentromers (Rottach et al., 2010) (Liu et al., 2013). Thus, I determined changes in UHRF1 localization to pericentromeric regions upon loss of TTD or SRA function in NIH3T3 cells.

To this end, I generated mutants of full-length UHRF1 in the aromatic cage of the TTD domain (Y188A, Y191A) and the NKR-finger of the SRA domain (Figure 3-2A) (Rottach et al., 2010) (Liu et al., 2013). As shown before (Figure 3-1), in a majority of NIH3T3 cells UHRF1 localizes to the pericentromeric heterochromatin, which is rich in H3K9me3 and 
DNA-methylation. I reasoned that UHRF1 co-localization with H3K9me3 and DNAmethylation would be lost due to the mutations.

It was shown before that pericentromeric heterochromatin can be visualized with 4',6'-diamidino-2-phenylindole (DAPI) staining (Matsuda and Chapman, 1991). I transiently expressed EGFP-labeled UHRF1 wild type or mutant protein in NIH3T3 cells and detected the pericentromeric heterochromatin with DAPI stain (Figure 3-2B). Monitoring these cells, I defined three phenotypes (Figure 3-2B). A dotted phenotype that is characterized by intense EGFP stained dots overlapping with the pericentromeric heterochromatin; an intermediate phenotype still showing these dots with an additional uniform EGFP background signal, and a diffuse phenotype, which only exhibited a uniformly distributed EGFP stain lacking any focal enrichment at pericentromeric regions. These categories enabled me to quantify changes in localization of UHRF1 in the nucleus of NIH3T3 cells upon loss of TTD or SRA domain function.

I found that $69 \%$ of cells transiently over-expressing EGFP-UHRF1 wild type protein showed a focal enrichment matching the DAPI staining pattern (Figure 3-2C). This ratio changed when cells were transiently expressing the mutant constructs. Only 36\% of TTD Y188A, Y191A mutant and 38\% of SRA R491A mutant protein showed the characteristic dotted pattern. At the same time, only $7 \%$ of cells transiently expressing wild type UHRF1 showed a diffuse nuclear EGFP signal phenotype. This diffuse fraction of cells exhibited a significant increase to 52\% for TTD mutant and 31\% for SRA mutant expressing cells. Almost all nuclei of cells expressing a protein carrying both mutations (Y188A, Y191A, R491A) displayed the diffuse phenotype (Figure 3-2D). Only $1 \%$ of cells still had a dotted phenotype of UHRF1 distribution and localization to the pericentromeric heterochromatin.

These results demonstrate that the binding contribution of both TTD and SRA are of great importance for the nuclear localization of UHRF1. I showed that both domains are contributing equally to target the protein to pericentromeric loci in NIH3T3 cells. We had previously shown that, in a PBR-bound state of UHRF1, the TTD aromatic cage is not interacting with H3K9me3 (Chapter 1.4.5) (Gelato et al., 2014). Thus, I was particularly interested in the contribution of this domain to the faithful localization of UHRF1. Comparing the dotted phenotype fractions, I determined that $30 \%$ of the NIH3T3 cells showed de-localization caused by the mutation of the TTD. Therefore, I expected that an effect on the UHRF1 localization in cells due to TTD interaction with the PBR would only be seen in this fraction of NIH3T3 cells. 
A

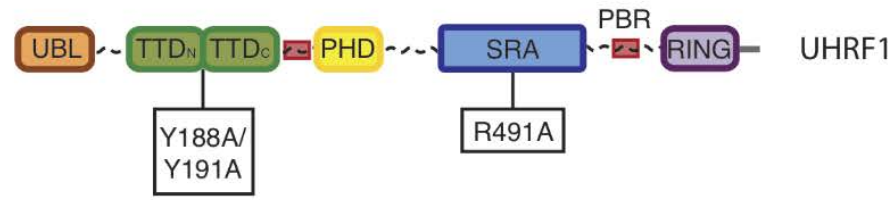

B

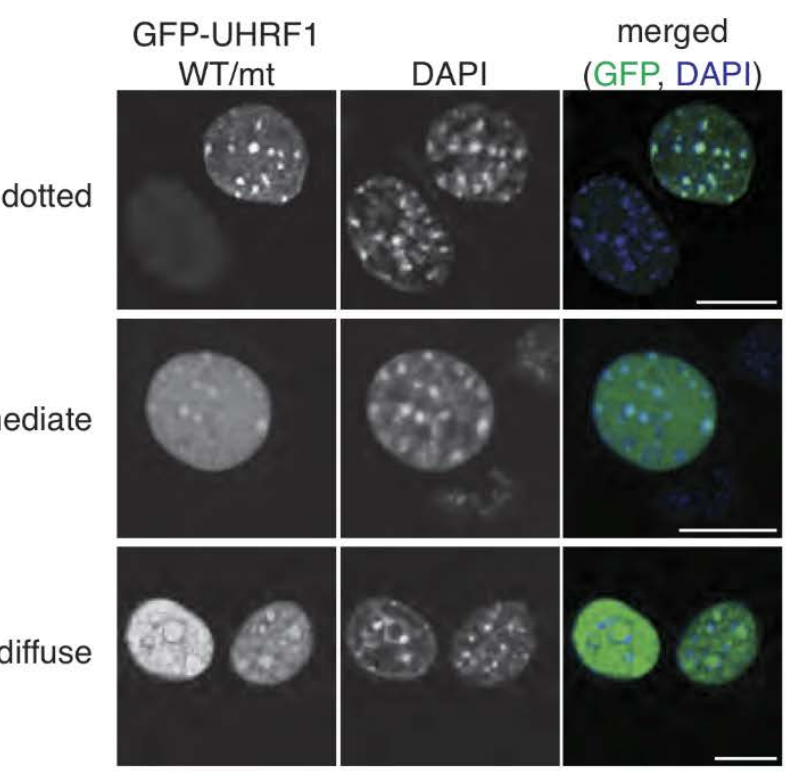

C

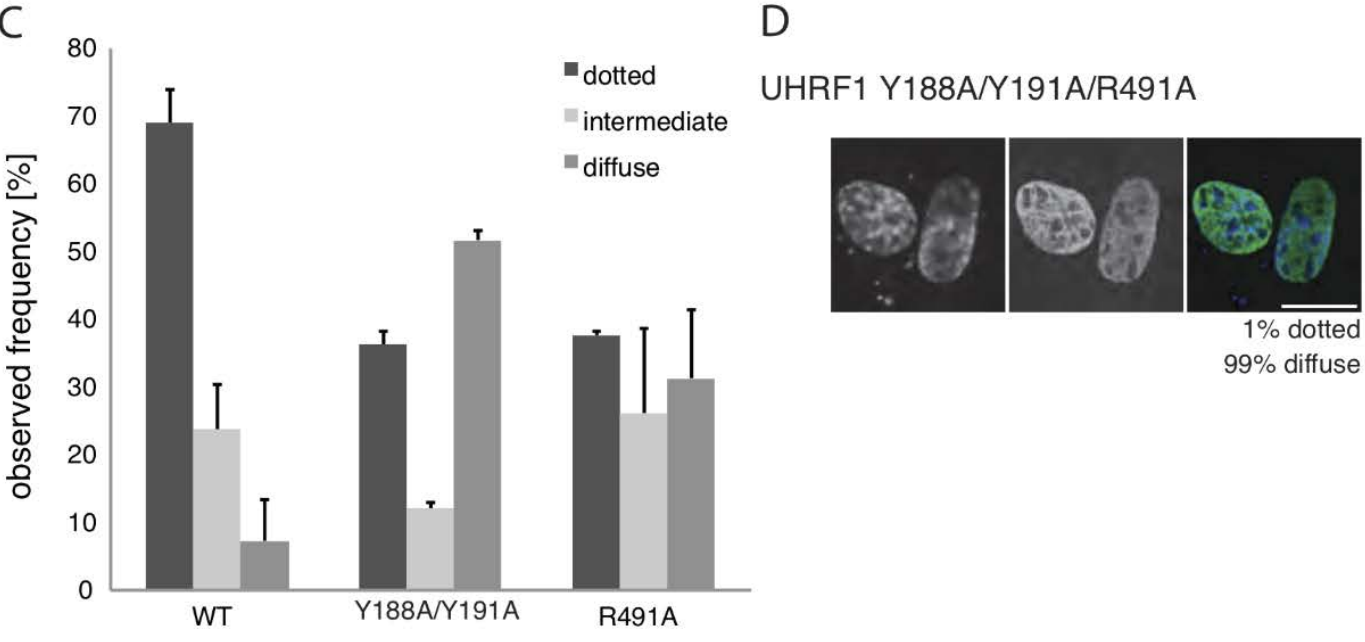

Figure 3-2 Nuclear localization of UHRF1 wild type and mutant in NIH3T3 cells

(A) Schematic representation of UHRF1 domain architecture the TTD (Y188A, Y191A) and SRA (R491A) mutations are indicated. (B) NIH3T3 cells were transfected with EGFP-UHRF1 wild type or mutant. DAPI was used to stain DNA. Images were obtained by fluorescence microscopy. Localization phenotype was determined by signal overlap of EGFP-UHRF1 wild type or mutant (green) with DAPI-dense regions (blue). Representative pictures of the three identified phenotypes (dotted, intermediate and diffuse) of EGFP-UHRF1 nuclear localization in NIH3T3 are shown. (C) UHRF1 wild type, TTD (Y188A, Y191A) and SRA (R491A) mutants were transiently expressed in NIH3T3 cells $(n>500)$. Relative quantification of cells exhibiting the EGFP-UHRF1 phenotypes shown in B. Error bars represent standard deviation of three independent experiments. (D) Representative fluorescence microscopy pictures of EGFP-UHRF1 TTD/SRA double mutant (Y188A, Y191A, R491A) with relative quantification of NIH3T3 cells ( $\mathrm{n}$ 500) exhibiting the EGFP-UHRF1 distribution classified in $B$. 
At the same time, only $69 \%$ of NIH3T3 cells showed a focal enrichment of EGFP-UHRF1 wild type protein to pericentromeric regions, while the other cells exhibited an intermediate or diffuse phenotype. Thus, release of the PBR from the TTD likely caused stronger focal enrichment to pericentromers in these $30 \%$ of cells, due to the increased binding of the domain to $\mathrm{H} 3 \mathrm{~K} 9 \mathrm{me} 3$.

\subsubsection{The PBR directs the TTD-dependent nuclear localization of UHRF1}

Our previous experiments showed that the PBR residues K644, K646, K648, R649, K650, S651 are essential to make contacts with the TTD peptide binding groove. In vitro binding studies of a PBR mutant (K644A, K646A, K648A, R649A, K650A, S651A) had shown increased interaction with the FAM-H3(1-15)K9me3 peptide (Gelato et al., 2014). Therefore, we reasoned that the PBR is released from the TTD in this context. I deduced that mutation of this region could lead to stronger co-localization of UHRF1 with H3K9me3 in vivo. Therefore, I determined the influence of the mutation of the PBR on the localization of UHRF1 to pericentromeric heterochromatin.

To this end, I generated an EGFP-tagged UHRF1 PBR mutant (K644A, K646A, K648A, R649A, K650A, S651A) to permanently release the PBR from the TTD (Figure 3-3A) (Gelato et al., 2014). I transiently expressed the PBR mutant EGFP-UHRF1 in NIH3T3 cells and determined the fractions of cells exhibiting the previously defined phenotypes (Chapter 3.1.2; Figure 3-2). I found that $67 \%$ of cells showed a focal enrichment of EGFP signal overlapping with the DAPI staining (Figure 3-3B). The remaining cells showed an intermediate or diffuse phenotype in $26 \%$ and $7 \%$ of the cells, respectively. Comparison of the co-localization of this PBR mutant and wild type UHRF1 with pericentromeric heterochromatin in NIH3T3 cells did not result in any significant difference in phenotype distribution (Figure 3-3B). Based on these findings, I deduced that the PBR mutation was not sufficient to change the subnuclear distribution of UHRF1.

Since the constant release of the PBR region from the TTD surface in context of full-length UHRF1 did not lead to any change in the nuclear localization, I decided to investigate the effects of increased TTD/PBR interaction. Our laboratory had previously shown that the interaction of the TTD with H3K9me3 peptides is blocked upon addition of PBR-RING region in vitro (Gelato et al., 2014). I reasoned that additional expression of PBR-RING together with UHRF1 should enforce the blocking of the TTD and thereby result in decreased localization of the protein to pericentromeric heterochromatin. 
A

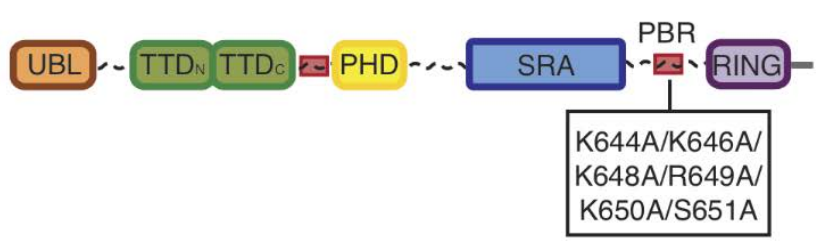

B

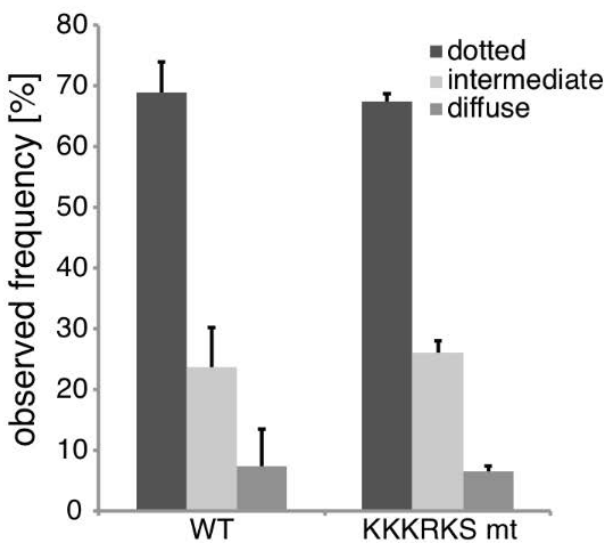

Figure 3-3 Nuclear localization of the UHRF1 PBR mutant in NIH3T3 cells

(A) Schematic representation of UHRF1 domain organization. The PBR mutant (K644A, K646A, K648A, R649A, K650A, S651A) is indicated. (B) EGFP-UHRF1 wild type or PBR mutant (K644A, K646A, K648A, R649A, K650A, S651A) were transiently expressed in NIH3T3 cells ( $\mathrm{r}$ 500). Relative quantification of cells that exhibited the defined EGFP-UHRF1 signal phenotypes for UHRF1 wild type and PBR mutant were plotted. Error bars represent standard deviation of three independent experiments.

I generated a wild type EGFP-tagged PBR-RING fragment as well as controls, EGFP alone and the previously described EGFP-PBR-RING mutant (K644A, K646A, K648A, R649A, K650A, S651A) (Figure 3-4A). I transiently expressed EGFP-tagged PBR-RING or controls together with mCherry-UHRF1 in NIH3T3 cells (Figures 3-4B). I used the previously defined phenotypes (Chapter 3.1.2; Figure 3-2), dotted, intermediate and diffuse, to define the changes in localization of mCherry-UHRF1 to pericentromeric heterochromatin (Figure 3-4C). I reasoned that for strong blocking of the TTD in trans the PBR-RING has to be overrepresented compared to UHRF1 to compete with its binding to H3K9me3 in NIH3T3 cells. Thus, I exclusively analyzed cells exhibiting a strong EGFP-PBR-RING signal to determine the influence of the overrepresented PBR region on the mCherryUHRF1 localization.

My results showed that an average of $58 \%$ of the cells exhibited a dotted localization phenotype of mCherry-UHRF1 when co-expressed with EGFP alone, while $29 \%$ were less enriched at pericentromers (intermediate) and 13\% had a diffuse appearance (Figure 3$4 \mathrm{E})$. The quantification of the different phenotypes upon co-expression of wild type EGFP-PBR-RING indicated that around 12\% of NIH3T3 cells shifted their mCherryUHRF1 distribution from the dotted to the intermediate or the diffuse phenotype (Figure 3-4D). In contrast, co-expressing the mutant EGFP-PBR-RING did not result in a significant change in localization (Figure 3-4D, E). Comparable results were obtained when co-expressing the NKR-finger SRA (R491A) mutant with EGFP-PBR-RING WT (Figure 3-4F). The fraction of NIH3T3 cells showing a dotted phenotype was reduced by $14 \%$ compared to the EGFP control. When mCherry-UHRF1 R491A was co-expressed with the EGFP-PBR-RING $\mathrm{mt}$ the fraction of dotted cells was only decreased by $5 \%$. 


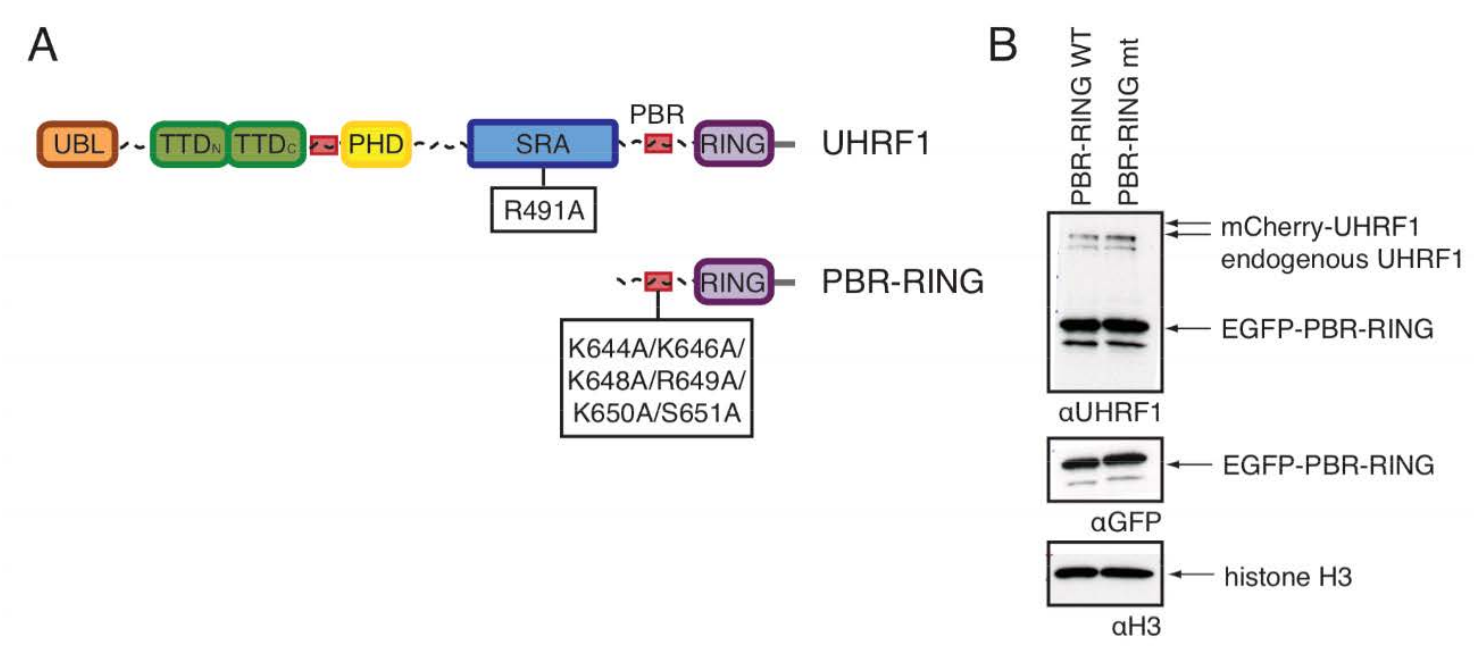

C

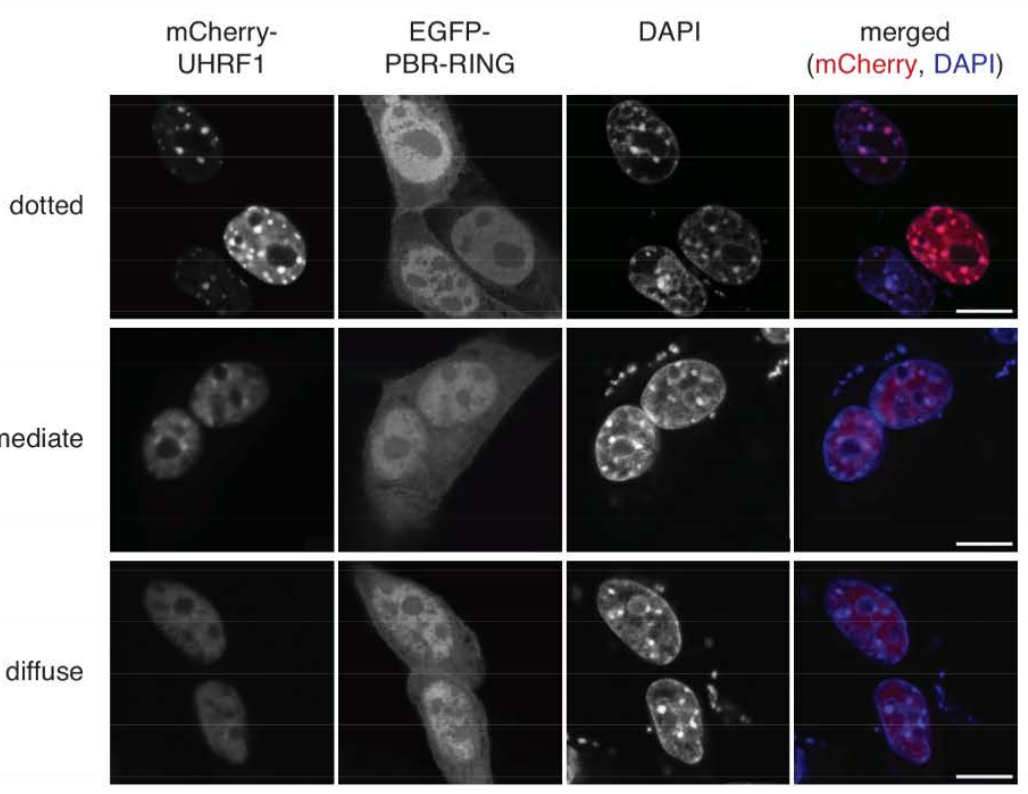

D

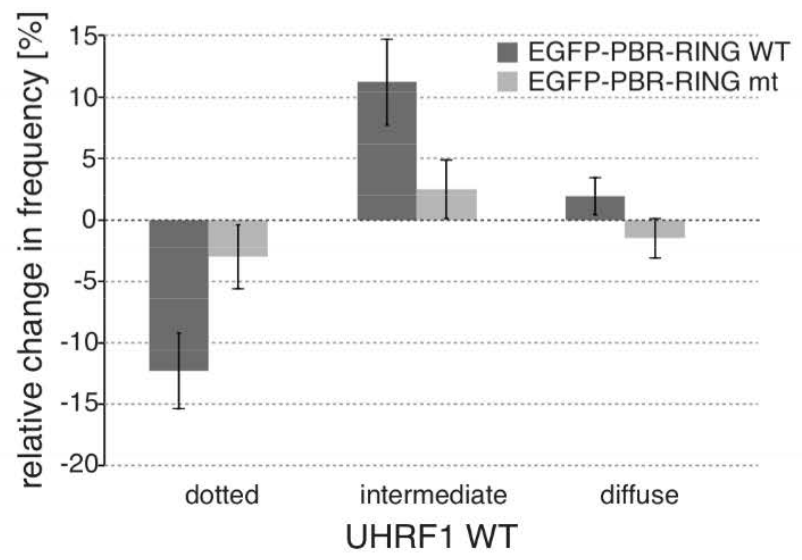

E

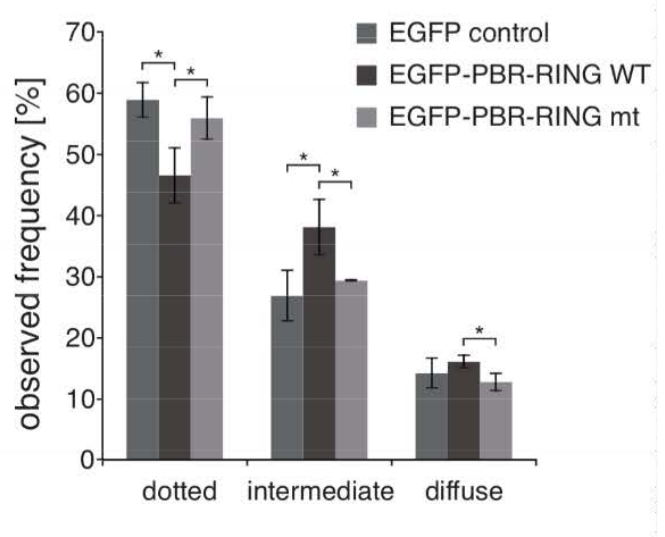




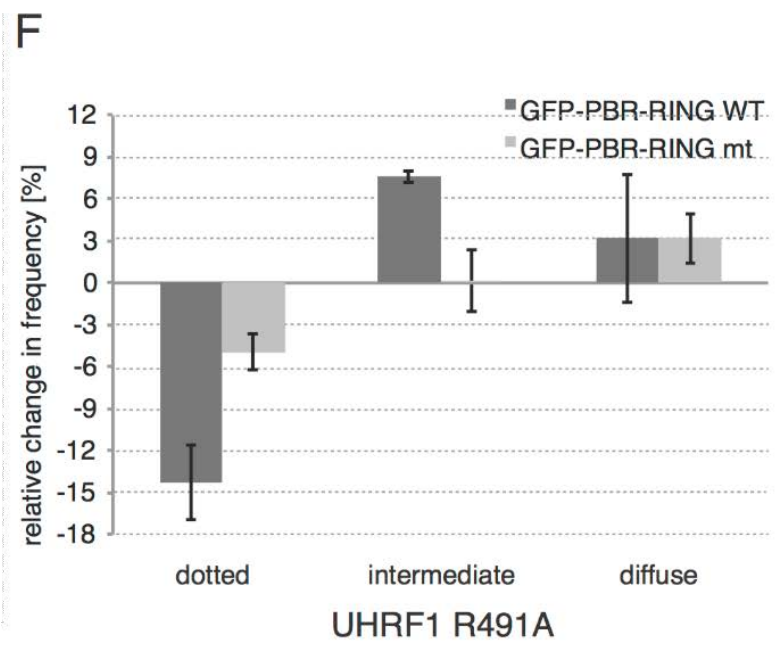

Figure 3-4 PBR-RING changes the nuclear localization of wild type UHRF1

(A) Schematic representation of UHRF1 domain organization highlighting the cassettes and mutants used in the experiments. (B) mCherry-UHRF1 together with EGFP-PBR-RING wild type (WT) or EGFP-PBR-RING K644A, K646A, K648A, R649A, K650A, S651A (mt) were transiently expressed in NIH3T3 cells. Total cell lysates were analyzed by western blotting. (C) mCherry-UHRF1 together with EGFP-PBR-RING wild type (WT) or EGFP-PBR-RING mutant (mt) were transiently expressed in NIH3T3 cells. Fluorescence signals were analyzed by confocal microscopy. Representative images of cells with different phenotypes observed at the indicated frequency $(\mathrm{n}>500)$ are shown. DAPI was used to stain DNA. Scale bar represents $10 \mu \mathrm{m}$. (D) Relative change in phenotype distribution of wild type mCherry-UHRF1 upon co-expression of EGFP-PBRRING wild type (WT) and mutant (mt) compared to only EGFP control. (E) Total frequencies of different nuclear localization phenotype of mCherry-UHRF1 expressed together with EGFP control, EGFP-PBR-RING wild type (WT) or EGFP-PBR-RING mutant $(\mathrm{mt})$ in NIH3T3 cells $(\mathrm{n}>500)$. (F) Relative change in phenotype distribution of mCherry-UHRF1 R491A mutant upon co-expression of EGFP-PBR-RING wild type (WT) and mutant $(\mathrm{mt})$ compared to only EGFP control. Error bars represent standard deviation of three independent experiments.

My earlier experiments determined that in a fraction of $30 \%$ of NIH3T3 cells the localization of UHRF1 to H3K9me3-rich pericentromers is dependent on the function of the TTD domain (Figure 3-2). Thus, almost half of the expected fraction of cells shifted their phenotype upon expression of the PBR-RING but not the controls. Obviously, the PBR region is important for the correct localization of UHRF1 to pericentromeric heterochromatin in NIH3T3 cells. In addition to that, similar shifts of mCherry-UHRF1 distribution were observed using the NKR-finger SRA (R491A) mutant. Thus, I reasoned that the PBR of UHRF1 is influencing the nuclear localization of the protein by its interaction with the TTD domain. 

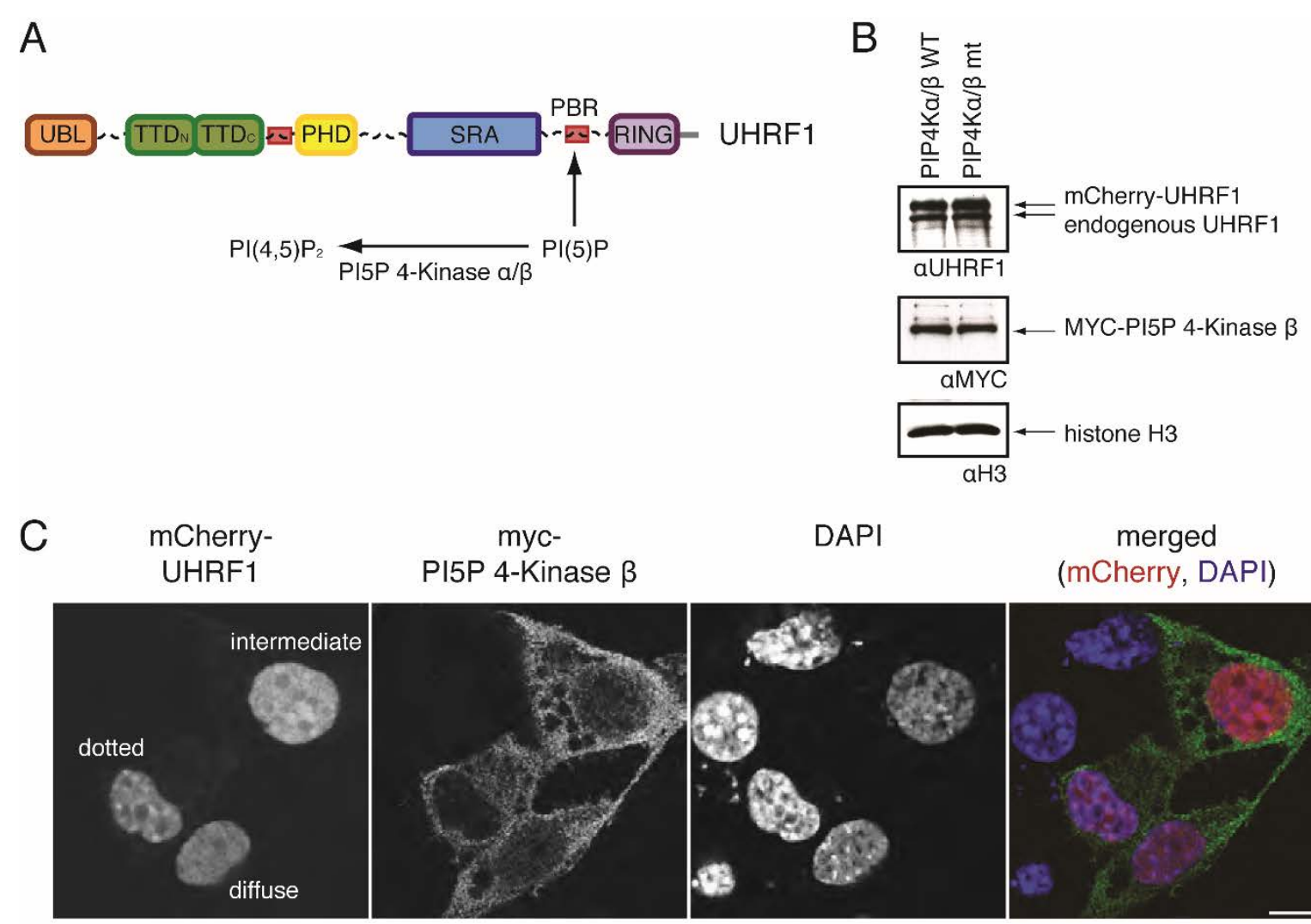

\section{merged
$($ mCherry, DAPI)}

D

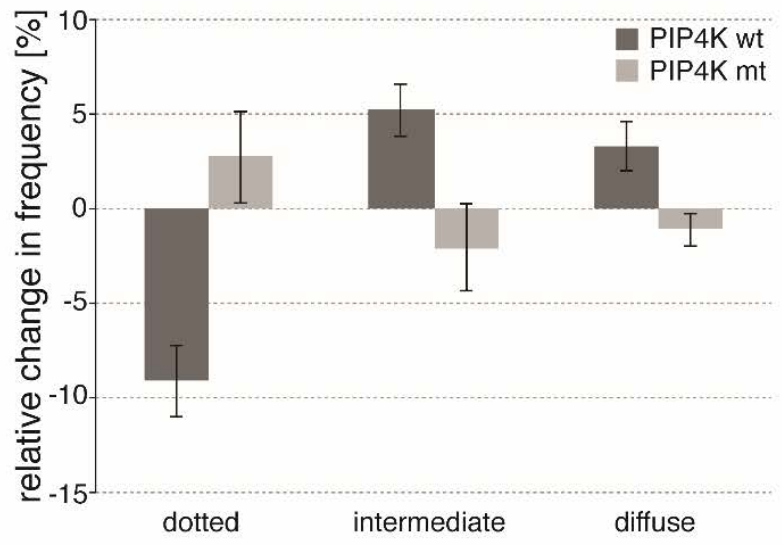

$E$

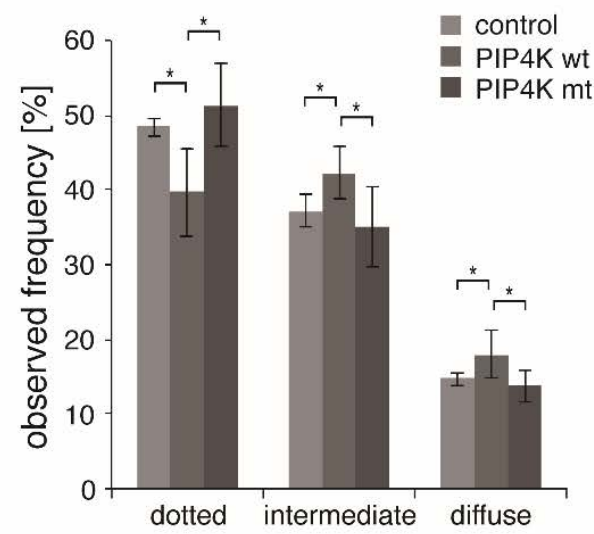

Figure 3-5 Influence of PI(5)P on the nuclear localization of UHRF1

(A) Schematic representation of UHRF1 domain organization. The potential PI(5)P interaction site is indicated. Also, the reaction that is catalyzed by PIP4K $\alpha / \beta$ is shown. PI(5)P is bound by the enzyme and phosphorylated to $\mathrm{PI}(4,5) \mathrm{P}_{2}$. (B) mCherry-UHRF1 together with myc-PIP4K $\beta /$ EE-PIP4K $\alpha$ wild type (WT) or myc-PIP4K $\beta$ (D278A)/EE-PIP4K $\alpha$ (G131L/Y138F) mutant (mt) were expressed in NIH3T3 cells. Total cell lysates were analyzed by western blotting. (C) NIH3T3 cells were transiently expressing mCherry-UHRF1 and mycPIP4K $\beta /$ EE-PIP4K $\alpha$ WT or myc-PIP4K $\beta /$ EE-PIP4K $\alpha$ mt proteins. Fluorescence signals were analyzed by confocal microscopy. Representative images of cells with different phenotypes observed at the indicated frequency $(n>500)$ are shown. DAPI was used to stain DNA. Scale bar represents $10 \mu \mathrm{m}$. (D) Relative change in phenotype distribution of wild type mCherry-UHRF1 upon expression of myc-PIP4K $\beta /$ EE-PIP4K $\alpha$ WT or myc-PIP4K $\beta$ /EE-PIP4K $\alpha$ mt compared to an empty vector control. (E) Total frequencies of different nuclear localization phenotypes of mCherry-UHRF1 expressed together with vector control, myc-PIP4K $\beta / \mathrm{EE}-\mathrm{PIP} 4 \mathrm{~K} \alpha$ WT or myc-PIP4K $\beta$ /EE-PIP4K $\alpha$ mt in NIH3T3 cells $(n>500)$. Error bars represent standard deviation of three independent experiments. Significance $\mathrm{p}<0.05$ is indicated by asterisks. 


\subsubsection{Nuclear PI(5)P directs TTD-dependent localization of UHRF1}

We demonstrated before that the PBR of UHRF1 interacts with the small molecule regulator, PI(5)P. This blocked the interaction of the PBR with the TTD and improved binding of UHRF1 to H3K9me3 peptides (Gelato et al., 2014). My previous experiments showed that UHRF1 nuclear localization is dependent on the TTD domain and is affected by the PBR region. Thus, I wanted to analyze the putative role of PI(5)P as a small molecule regulator of UHRF1 sub-nuclear localization.

It was reported previously that the pool of nuclear PI(5)P is regulated by PI(5)P 4-kinase $\beta$. The transient over-expression of PI(5)P 4-kinase $\beta$ in MEL cells led to reduced nuclear PI(5)P levels (Jones et al., 2006). PI(5)P 4-kinase $\beta$ is also essential for the subnuclear localization of PI(5)P 4-kinase $\alpha$. Both enzymes metabolize PI(5)P to PI(4,5)P $\mathrm{P}_{2}$ but PI(5)P 4kinase $\alpha$ was shown to be 2,000-fold more active compared to PI(5)P 4-kinase $\beta$ (Bultsma et al., 2010). Therefore, I decided to transiently express both isoforms of PI(5)P 4-kinases (PIP4Ks) to effectively reduce the nuclear level of the co-factor in NIH3T3 cells (Figure 3$5 \mathrm{~A})$.

I expressed wild type and mutant EE-PIP4K $\alpha$ and Myc-PIP4K $\beta$ together with mCherryUHRF1 in NIH3T3 cells (Figure 3-5B) and analyzed the resulting phenotype of mCherryUHRF1 signal distribution (Figure 3-5C). I evaluated the experiment comparing the effects of the wild type as well as mutant PIP4K $\alpha$ (G131L, Y138F) and Myc-PIP4K $\beta$ (D278A) to a control expressing empty vector. I observed a shift from a dotted to an intermediate or even diffuse distribution in an average of $9 \%$ of cells upon PIP4K $\alpha / \beta$ expression (Figure 3-5D, E). This significant shift in UHRF1 localization was not observed when coexpressing the PIP4K $\alpha$ (G131L, Y138F) / $\beta$ (D278A) mutant proteins.

My results show that UHRF1 subnuclear localization changed upon PIP4K $\alpha / \beta$ overexpression in the NIH3T3 cells. This suggested that the lack of PI(5)P in this context might responsible for enhanced blocking of the TTD by the PBR and reduced interaction of the TTD with H3K9me3. Therefore, I reasoned that PI(5)P, a regulator of the TTD/PBR interaction in vitro, influences the nuclear localization of UHRF1 to H3K9me3-rich pericentromeric heterochromatin in NIH3T3 cells. 


\subsubsection{Novel cyclic peptides regulate the TTD/ PBR interaction}

It was reported that UHRF1 is highly expressed in different human cancerous tissues. This observation was linked to hypermethylation of tumor suppressor genes (Alhosin et al., 2011; Bronner et al., 2007; Unoki et al., 2009; Unoki et al., 2010). Therefore, it was proposed that UHRF1 might constitute an interesting target for new approaches in cancer therapy (Unoki et al., 2009). A recent study identified small molecule regulators of the UHRF1 SRA domain, which can alter DNA-methylation in cells (Myrianthopoulos et al., 2016). I found that the localization of UHRF1 in vivo is not only dependent on the SRA domain but also on the TTD domain. I showed that the interaction of the TTD with H3K9me3-rich pericentromeric heterochromatin is affected by a small molecule regulator (PI(5)P) targeting the intramolecular interaction of the TTD with the PBR. Thus, I wanted to identify small molecules that have the potential to modulate the UHRF1 TTD/PBR interaction.

In collaboration with the laboratory of Shiori Umemoto (Laboratory of Hiroaki Suga, University of Tokyo, Japan) a library of small random peptides was screened with the RaPID method (Random non-standard Peptide Integrated Discovery) for potential cyclic regulatory peptides (Hayashi et al., 2012). The candidate selection process of this method involves two steps, a negative and a positive selection. A PBR (R649A, K650A, S651A) mutant was used to perform negative selection to eliminate peptides that are not interacting on the basis of the PBR sequence or the PBR/TTD interaction. In the positive selection step, the remaining peptide candidates were screened for interaction with wild type UHRF1 to specifically select peptides interacting with the PBR or the PBR-bound conformation of the protein (Chapter 2.3.3.4). This screening procedure identified ten candidate peptides, L1-L5 and D1-D5 (Figure 3-6A)

I characterized these candidate peptides to define small molecules specifically affecting the interaction between the PBR and the TTD. I first analyzed the affinity of the candidate peptides to UHRF1 wild type full-length protein using a peptide pull down assay, in which I used the immobilized peptides as baits for wild type UHRF1 protein (Figure 3$6 \mathrm{~B})$. The results showed that UHRF1 was retained with five of the ten candidate peptides, namely L1, L4, D3, D4 and D5. For additional validation, I set up quantitative analysis using fluorescence polarization measurements (Table 3-1). Only the peptides L1, L3, L4, D1, D4 and D5 exhibited binding to UHRF1 full-length protein in this assay (Figure 3-6C). Both techniques identified L1, L4, D4 and D5 as potential candidates for the regulation of the TTD / PBR interaction. 
A

\section{L1 yAAIWVKHHYRELVKN-C \\ L2 ySYRIKHHTTKWTKTP-C}

L3 ySWRIKGHHNRWKQTP-C

L4 yRYRVRTHNSRWTVLS-C

L5 yKSQVRHHPSTKQNNY-C

D1 yRYWTHTHKSHKTTIP-C

D2 yTWLDHHDWHRYTRLA-C

D3 yTYKSLIVKNHKRHLS-C

D4 yPYNRILIKRHRVKII-C

D5 yPYVLIYVNNHKRPSR-C
B

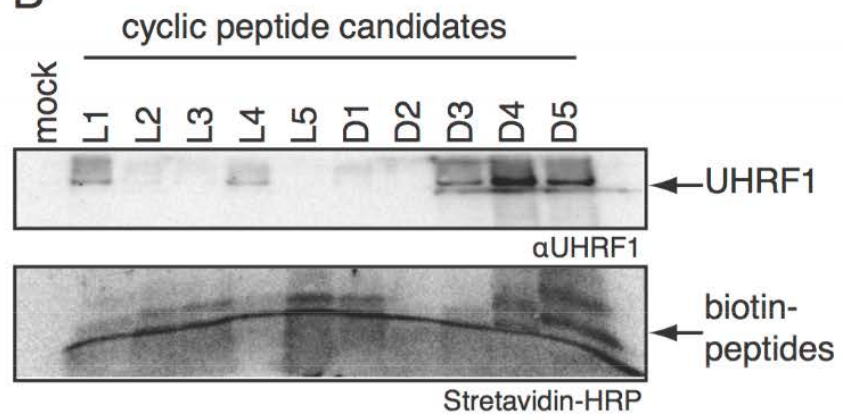

C

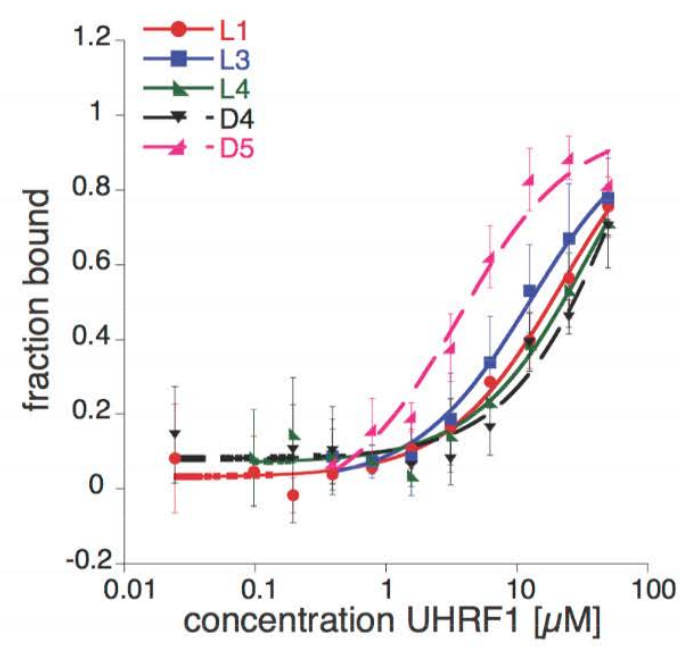

$E$
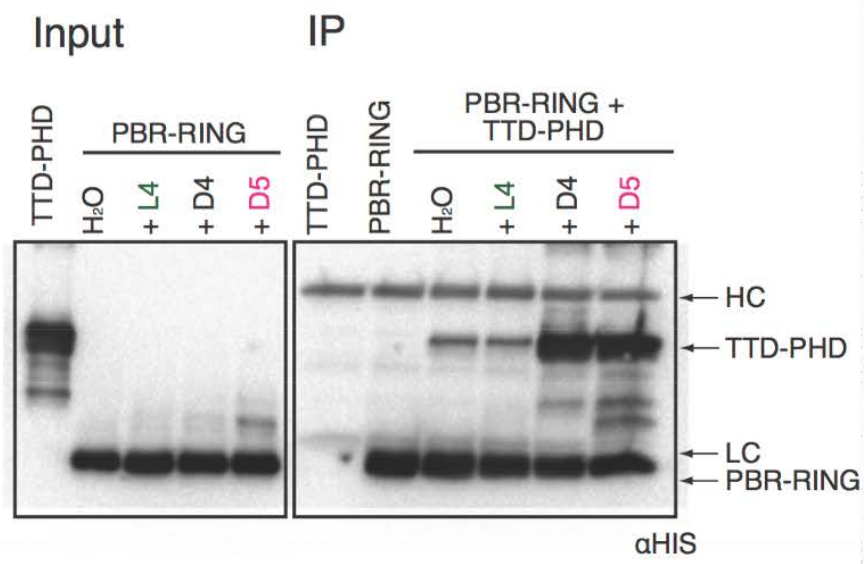

Figure 3-6 Regulation of the TTD/ PBR interaction by cyclic peptides

(A) Amino acid sequences of cyclic peptide candidates resulting from the RaPID screening procedure performed in collaboration with Shiori Umemoto (Laboratory of Hiroaki Suga, University of Tokyo) (B) Peptide pull down assay with full-length wild type UHRF1. The biotin conjugated cyclic peptide candidates were immobilized on beads and incubated with UHRF1. Material retained after washing was analyzed by western blot. $\alpha$ UHRF1 signal (upper panel) and peptide loading control Streptavidin HRP (lower panel). Interaction of wild type UHRF1 (C) and UHRF1 PBR-RING fragment (D) with the indicated fluorescein labeled cyclic peptides was analyzed in fluorescence polarization binding experiments. (E) UHRF1 PBR-RING region was pre-incubated with L4, D4, D5 peptides or solvent control (Input) and coupled to beads using a specific antibody. Beads conjugated with the indicated peptide/PBR-RING mix and control beads were then incubated with UHRF1 TTD-PHD (Input) and used in a co-immunoprecipitation assay (IP). The material retained after washing was analyzed by western blot using $\alpha$-His antibody. 
Next, I wanted to select cyclic peptides interacting with the C-terminal PBR-RING region of UHRF1 to find potential regulators of the PBR interaction with the TTD domain. Fluorescence polarization binding experiments with PBR-RING validated three of the above peptides L4, D4 and D5 (Figure 3-6D), as interaction partners of the PBR region. The peptide D5 showed the strongest interaction with PBR-RING $(\mathrm{Kd}=6.4 \mu \mathrm{M})$, while L4 and $\mathrm{D} 4$ were bound with a lower affinity $(\mathrm{Kd}=15 \mu \mathrm{M})$.

To determine the influence of the selected candidate peptides on the TTD/PBR interaction, I set up a domain co-immunoprecipitation experiment (Figure 3-6E). It had already been shown that the TTD but not the PHD domain co-precipitates with the PBRRING region (Gelato et al., 2014). In my experiment I used the PBR-RING and TTD-PHD regions of UHRF1. I pre-incubated the PBR-RING domain with a five-fold molar excess of L4, D4, D5 peptide candidates or the equivalent amount of peptide solvent and coupled it to beads using $\alpha$-UHRF1 antibody. Subsequently, I added the TTD-PHD for coprecipitation. The results showed that TTD-PHD was co-precipitated by PBR-RING that was incubated with peptide solvent but not by the antibody control, suggesting a direct interaction of the two UHRF1 regions. This interaction of PBR-RING with the TTD-PHD did not change upon addition of 5-fold molar excess of L4 peptide. In contrast, the addition of D4 and D5 peptides enforced the co-precipitation of TTD-PHD, arguing for strengthened interaction with PBR-RING. Thus, these candidate peptides might enforce the intramolecular interactions of UHRF1 and inhibit binding of the TTD to H3K9me3.

Table 3-1 binding affinity of UHRF1 and PBR-RING to cyclic peptide candidates

\begin{tabular}{ccc} 
(apparent Kd values as determined by fluorescence polarization measurements in $\mu \mathrm{M}$ ) \\
\hline L1 & UHRF1 & PBR-RING \\
\hline L2 & $21.5 \pm 4.6$ & $38.9 \pm 23.2$ \\
L3 & - & - \\
L4 & $12.4 \pm 2.7$ & $28.2 \pm 11.6$ \\
L5 & $27.7 \pm 13.9$ & $15.3 \pm 6.4$ \\
D1 & $\mathrm{ND}$ & $\mathrm{ND}$ \\
D2 & $36.1 \pm 21.6$ & $88.1 \pm 56.7$ \\
D3 & $74.1 \pm 33.2$ & $\mathrm{ND}$ \\
D4 & $\mathrm{ND}$ & $45.6 \pm 18.8$ \\
D5 & $54.4 \pm 45.0$ & $15.1 \pm 7.6$ \\
\hline
\end{tabular}




\subsection{NP95 binding behavior is dependent on its linker regions}

UHRF1 is a factor responsible for the recruitment of DNMT1, G9a and HDAC1 to pericentromeric heterochromatin during S-phase of the cell cycle. Therefore, the proper nuclear localization of the protein, which is facilitated by the TTD and the SRA domain, is essential for the maintenance of epigenetic marks through continuous cell divisions. Others and we pointed out the importance of inter-domain linker regions in the regulation of UHRF1 interaction with the H3 tail (reviewed in (Tauber and Fischle, 2015)). At least two linker regions of the protein have been described that can influence the binding to H3K9me3 by differential interactions with the TTD (Figure 3-7A). The TTDPHD linker was shown to establish a multivalent binding mode of UHRF1 TTD and PHD domains binding to $\mathrm{H} 3 \mathrm{~K} 9 \mathrm{me} 3$ and the unmodified $\mathrm{N}$-terminus of $\mathrm{H} 3$, respectively (Arita et al., 2012; Cheng et al., 2013; Rothbart et al., 2013; Xie et al., 2012). We uncovered that the second linker region, the PBR, blocks the interaction of the TTD with H3K9me3. Consequently, UHRF1 did not specifically bind to H3K9me3 peptides in vitro (Gelato et al., 2014). My previous results showed that this PBR dependent blocking of the TTD/H3K9me3 interaction is relevant for the correct sub-nuclear localization of UHRF1.

The structure and function of chromatin interacting domains of UHRF1 proteins of different vertebrate species are highly conserved (Bronner et al., 2007). Given the importance of the PBR region for the accurate localization of UHRF1, I reasoned that the inter-domain linker regions connecting these domains are also essential for the specific binding of UHRF1 proteins to chromatin modifications. To determine amino acid conservation in these regions, I performed sequence comparison of the two linkers between different representative species.

\subsubsection{The primary structures of TTD-PHD linker and PBR domains are highly conserved}

The sequences of UHRF1 orthologues from various species are highly conserved in vertebrates (Chapter 1.4.2) (Bronner et al., 2007). Thus, I analyzed the evolutionary conservation of the regulatory linker regions by comparison of amino acid sequences of a cross section of vertebrate species using the PRALINE alignment tool (http: / / zeus.few.vu.nl/ programs/ pralinewww/). 


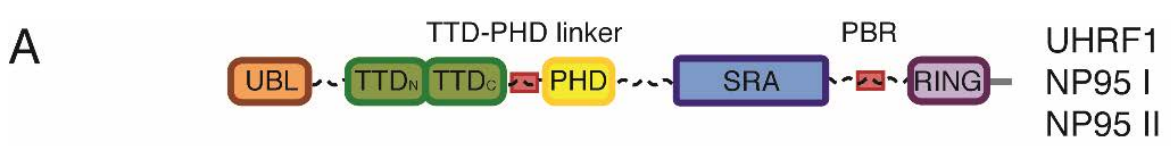

B

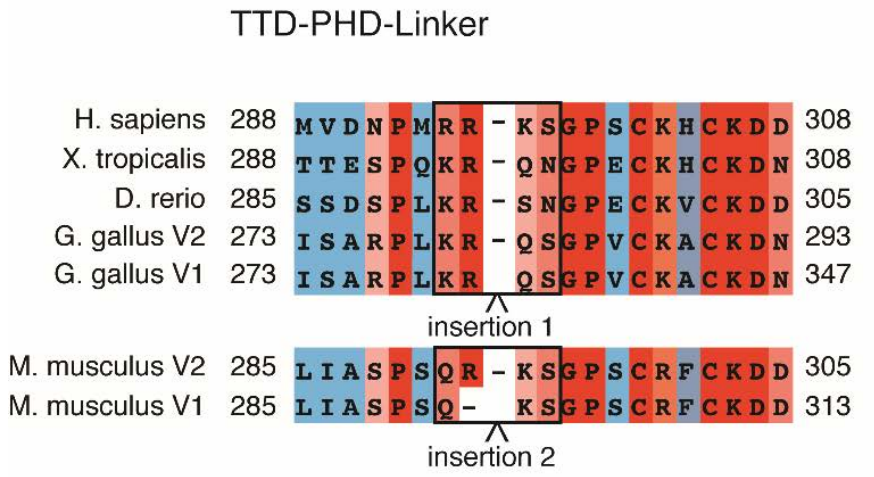

C

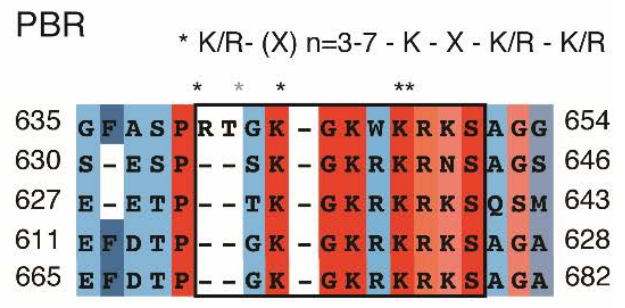

625 E - Q G P S S S K T G K S K Q K T GP 644 633 E - Q G P S S S K T G K S K Q K S T G P 652

insertion 1: EYCIFLVCIVVLSCDVLLIIFEHQNSKTLAAVTPLTICNASLHFLECCFELLIG

insertion 2: PPPALRNTG

Unconserved 012345678910 Conserved

Figure 3-7 Sequence conservation of TTD-PHD linker and PBR

(A) Schematic representation of the common domain structure of UHRF1 proteins of different species. (B) Multiple sequence alignments of sequence stretches within the TTD-PHD linker region are shown. Sequences of insertions found in splicing variants of mouse and chicken proteins are given. (C) Multiple sequence alignments of sequence stretches within the PBR region of UHRF1 are shown. A putative PIP binding consensus motif within the PBR is specified. All alignments were prepared using the PRALINE alignment tool (http://zeus.few.vu.nl/programs/pralinewww/). Numbers correspond to amino acid positions at the beginning and end of the respective protein sequences of the different species. UHRF1 amino acid sequences according to NCBI: Homo sapiens, NP_001276981.1; Mus musculus v1, NP_001104550.1, Mus musculus v2, NP_001104548.1, Gallus gallus v1, XP_418269.4, Gallus gallus v2, XP_004949013.1; Xenopus laevis, F6UA42.2; Danio rerio, NP_998242.1.

I found that the TTD-PHD linker and the PBR show particular sequence conservation. The TTD-PHD linker has a conserved basic patch (R-R-K, K-R, R-K) that is accompanied by an adjacent Serine residue (Figure 3-7B). In UHRF1, a R-K-S motif in the linker was shown to make contacts with the TTD surface and thus establish multivalent binding of TTD and PHD to a single H3 tail (Arita et al., 2012; Rothbart et al., 2013). This essential R-K-S motif is only fully conserved in mouse (M. musculus). In chicken (G. gallus) and zebrafish (D. rerio), I found comparable sequences K-R-Q-S and K-R-S, respectively. Only in tropical clawed frog (X. tropicalis), the UHRF1 sequence does not comprise a similar sequence motif and only a basic patch is conserved (K-R-Q-N). Our analysis identified a second isoform of the protein in mouse and chicken that carries an insertion in the TTD-PHD linker. The large 54 amino acid insertion in chicken UHRF1 is surprisingly rich in cysteine and histidine residues and thus might include an alternative extension of the PHD zincfinger (Figure 3-7B, insertion 1). In mouse UHRF1 the insertion of 9 amino acids (P-P-P-AL-R-N-T-G) in NP95 isoform I (NP95 I) disrupts the conserved RKS motif in the TTD-PHD linker region (Figure 3-7B, insertion 2). Irrespective of these differences UHRF1 and NP95 
I have been used interchangeable in several studies (Bostick et al., 2007; Karagianni et al., 2008; Nady et al., 2011; Nishiyama et al., 2013).

The PBR exhibits a large basic patch with strong conservation of a sequence of basic residues, K-G-K-X-K-X-K-S (Figure 3-7C). Nevertheless, only the human (H. sapiens) basic patch sequence fit the described putative PIP recognition motif $K / R-(X)_{3-7}-K-X-K / R-K / R$ (Figure 3-7C) (Lewis et al., 2011). Similar to the TTD-PHD linker, an adjacent serine residue accompanies the basic patches in all sequences analyzed. Strikingly, the amino acid conservation in all sequences mainly involved residues that were shown to be essential for the interaction of the TTD-PHD linker or the PBR with the TTD (Arita et al., 2012; Gelato et al., 2014; Rothbart et al., 2013).

\subsubsection{The PBR region does not regulate NP95 binding to H3K9me3}

\subsubsection{Comparison of UHRF1 and NP95 binding to the $\mathrm{H} 3$ tail using FP}

In mouse, I found two splicing variants of UHRF1, NP95 I and II. NP95 I TTD-PHD linker comprised a 9 amino acid insertion disrupting the described characteristic R-K-S motif. Due to this linker insertion, I hypothesized that the binding behavior of NP95 I differs from UHRF1 as well as NP95 II. At the same time, I expected that UHRF1 and NP95 II exhibit similarities in their $\mathrm{H} 3 \mathrm{~K} 9 \mathrm{me} 3$ binding.

I used these peptides in a fluorescence polarization read out, to compare the binding of UHRF1, NP95 I and NP95 II to the H3 tail and to determine how the different linker regions affect the binding of the TTD to $\mathrm{H} 3 \mathrm{~K} 9 \mathrm{me} 3$, the PHD interaction with the $\mathrm{H} 3$ unmodified N-terminus and the multivalent binding mode of both domains. Our previous study revealed $\mathrm{H} 3$ tail peptides that target specific domains of UHRF1 (TTD, PHD) or combinations of these (TTD+PHD) (Table 3-2) (Gelato et al., 2014). All peptides used for fluorescence polarization experiments comprised the amino acids 1 to 15 of the histone H3 tail. The FAM-H3(1-15)K9me3 peptide was additionally tri-methylated at the $\mathrm{K} 9$ residue and labeled with FAM at the very N-terminus. This peptide was exclusively bound by the TTD and not by the PHD domain. The H3(1-15)unmod-FAM peptide carried no modifications and was labeled with FAM at its C-terminus. It was bound by the PHD but not by the TTD. The third peptide H3(1-15)K9me3-FAM was tri-methylated at the K9 and labeled with FAM at the C-terminal end and hence was able to bind to both TTD and PHD. A table with all measured apparent Kd values can be found in table 3-3. 
Table 3-2 Peptides used for fluorescence polarization and binding domains of UHRF1/ NP95

\begin{tabular}{ll}
\hline Peptide & Binding domain \\
\hline FAM-H3(1-15)K9me3 & TTD \\
H3(1-15)unmod-FAM & PHD \\
H3(1-15)K9me3-FAM & TTD, PHD \\
\hline
\end{tabular}

Table 3-3 binding affinity of all fragments and mutants of UHRF1 and NP95 to indicated peptides (apparent $\mathrm{Kd}$ values as determined by fluorescence polarization measurements in $\mu \mathrm{M}$ )

\begin{tabular}{|c|c|c|c|c|c|}
\hline Protein & Cassette & Mutation & FAM-H3K9me3 1-15 & H3 unmod 1-15 -FAM & H3K9me3 1-15 -FAM \\
\hline \multirow[t]{6}{*}{ UHRF1 } & full-length & & ND & $16.1 \pm 2.3$ & $10.7 \pm 1.9$ \\
\hline & & insertion $\mathrm{mt}$ & ND & $13.9 \pm 1.9$ & $16.9 \pm 2.8$ \\
\hline & TTD & & $3.1 \pm 0.9$ & ND & $1.9 \pm 0.7$ \\
\hline & TTD-PHD & & $15.9 \pm 4.6$ & $1.9 \pm 0.9$ & $1.3 \pm 0.5$ \\
\hline & & insertion $\mathrm{mt}$ & $64.9 \pm 103.5$ & $1.3 \pm 0.6$ & $2.2 \pm 0.8$ \\
\hline & PBR-RING & & ND & ND & ND \\
\hline \multirow[t]{2}{*}{ NP95 } & TTD & & $3.4 \pm 0.5$ & ND & $2.6 \pm 0.7$ \\
\hline & PBR-RING & & ND & ND & ND \\
\hline \multirow[t]{12}{*}{ NP95 I } & full-length & & ND & $17.4 \pm 2.1$ & $9.9 \pm 0.6$ \\
\hline & & PPP-AAA & ND & $18.6 \pm 3.0$ & $10.3 \pm 1.2$ \\
\hline & & $\mathrm{R}-\mathrm{A}$ & ND & $14.8 \pm 2.8$ & $19.9 \pm 2.5$ \\
\hline & & KS-AA & ND & $8.7 \pm 1.7$ & $5.8 \pm 1.1$ \\
\hline & & $3 \mathrm{KA}$ & ND & $26.5 \pm 6.8$ & $23.7 \pm 4.2$ \\
\hline & & $4 \mathrm{KA}$ & ND & $18.6 \pm 3.7$ & $14.8 \pm 2.2$ \\
\hline & TTD-PHD & & ND & $2.9 \pm 0.4$ & $1.9 \pm 0.2$ \\
\hline & & YY-AA & ND & $10.3 \pm 1.2$ & $12.1 \pm 1.1$ \\
\hline & & DE-AA & ND & $39.5 \pm 8.9$ & $46.9 \pm 12.1$ \\
\hline & & PPP-AAA & $11.1 \pm 2.2$ & $3.8 \pm 0.7$ & $4.5 \pm 0.95$ \\
\hline & & R-A & $5.0 \pm 0.7$ & $2.2 \pm 0.3$ & $2.4 \pm 0.3$ \\
\hline & & KS-AA & ND & $2.5 \pm 0.5$ & $2.5 \pm 0.5$ \\
\hline \multirow[t]{8}{*}{ NP95 II } & full-length & & $39.8 \pm 11.2$ & $37.0 \pm 14.9$ & $24.8 \pm 2.7$ \\
\hline & & RKS-AAA & $29.3 \pm 6.3$ & $12.1 \pm 2.1$ & $14.3 \pm 2.3$ \\
\hline & & $3 \mathrm{KA}$ & ND & $28.5 \pm 9.7$ & $43.9 \pm 12.2$ \\
\hline & & $4 \mathrm{KA}$ & ND & $33.1 \pm 11.3$ & $29.2 \pm 8.6$ \\
\hline & TTD-PHD & & $21.7 \pm 6.1$ & $11.1 \pm 1.5$ & $6.3 \pm 0.65$ \\
\hline & & YY-AA & ND & $10.4 \pm 2.5$ & $13.6 \pm 3.2$ \\
\hline & & DE-AA & $16.3 \pm 3.5$ & ND & $27.7 \pm 3.1$ \\
\hline & & RKS-AAA & $40.0 \pm 11.6$ & $4.4 \pm 0.75$ & $1.6 \pm 0.5$ \\
\hline
\end{tabular}




\subsubsection{Full-length wild type UHRF1 and NP95 I show similar H3 tail binding}

Our previous work had identified discrepancies between the H3K9me3 binding specificity of the TTD-PHD region and the full-length UHRF1 protein (Gelato et al., 2014). We found that the binding to H3K9me3 in context of the full-length protein is impaired by the interaction of the TTD with the PBR region. The PBR region is an inter-domain linker region in the C-terminus of the protein. It was shown to interact with the TTD surface of UHRF1 and blocked the interaction with H3K9me3 (Gelato et al., 2014). As described before, sequence comparison of the PBR region in mouse and human UHRF1 revealed high similarity (Chapter 3.2.1). This suggested a similar role of the PBR in human UHRF1 and the two murine variants. To detect the potential influence of the PBR on the H3K9me3 binding, I compared the $\mathrm{H} 3$ tail binding behavior of the full-length proteins of NP95 I and II with UHRF1 (Figure 3-8A).

First, I investigated the $\mathrm{H} 3$ tail binding behavior using a peptide pull down assay with C-terminally biotin-labeled H3(1-20) unmodified or H3(1-20)K9me3 peptides. We had already described that in a similar assay with the wild type full-length UHRF1, the protein is similarly recovered by both peptides (Gelato et al., 2014). Similar to UHRF1, my experiments showed that murine variant NP95 I was recovered equally well by unmodified and $\mathrm{K} 9$ tri-methylated $\mathrm{H} 3$ tail peptides (amino acids 1-20) (Figure 3-8B). In contrast, NP95 II showed clear preference for the K9 tri-methylated peptide.

To analyze the binding contribution of the single domains in context of the full-length proteins, I performed fluorescence polarization binding experiments with the described peptides targeting TTD, PHD or TTD and PHD. As previously reported, UHRF1 bound to the C-terminally FAM tagged peptides regardless of their K9 methylation, while it failed to interact with the FAM-H3(1-15)K9me3 peptide. This clearly showed that the TTD is not contributing to peptide binding in this context (Figure 3-8C) (Gelato et al., 2014). Fluorescence polarization experiments with NP95 I and the defined H3 tail peptides revealed a binding behavior comparable to UHRF1, as already suggested by the peptide pull down assay (Figure 3-8B, D). NP95 I bound to the C-terminally FAM-labeled unmodified

$(\mathrm{Kd}=17.4 \mu \mathrm{M})$ and $\mathrm{K} 9$ trimethylated $(\mathrm{Kd}=9.9 \mu \mathrm{M}) \mathrm{H} 3$ tail peptides. In contrast, NP95 II moderately bound to all peptides regardless of their label or modification. Based on these findings, I reasoned that not only PHD but also the TTD is contributing to the peptide interaction in NP95 II. 
Contrary to my initial hypothesis, UHRF1 binding to the H3 tail differed from NP95 II, despite their strong sequence similarity. Instead, NP95 I and UHRF1 exhibited a similar H3K9me3 binding behavior. Both proteins did not interact with FAM-H3K9me3 peptide, suggesting that the TTD domain is blocked in context of the full-length wild type proteins and does not contribute to the $\mathrm{H} 3$ tail interaction. Given the sequence similarities of UHRF1 and NP95 in the PBR region, I reasoned that the block of the TTD might be conserved in NP95 I but not in NP95 II.

A

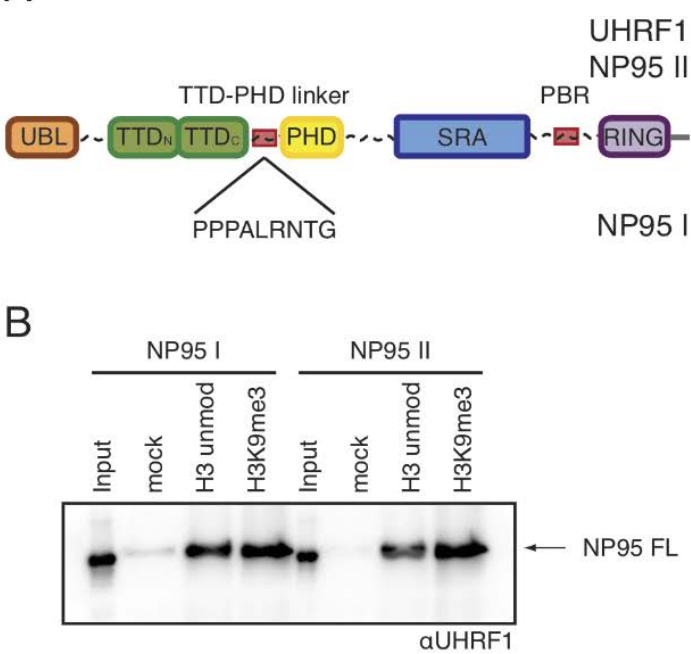

D

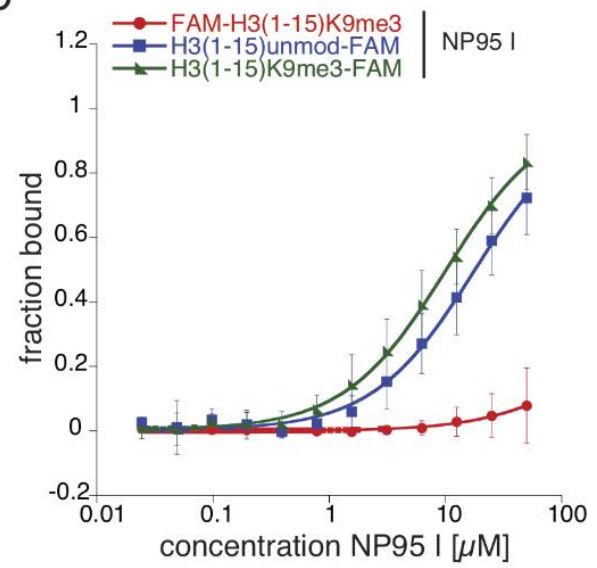

C

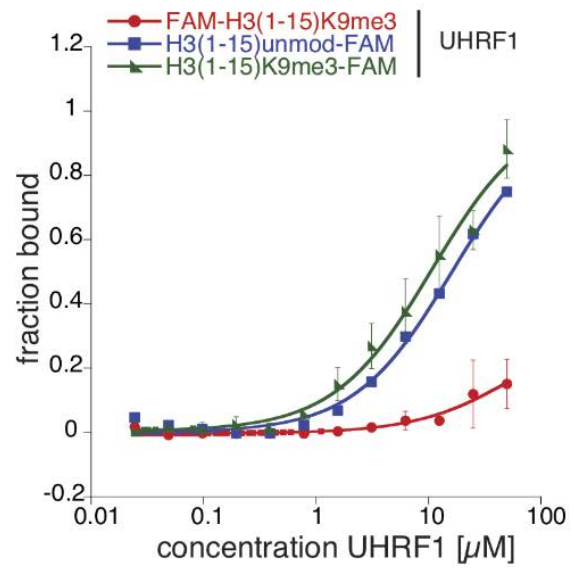

$\mathrm{E}$

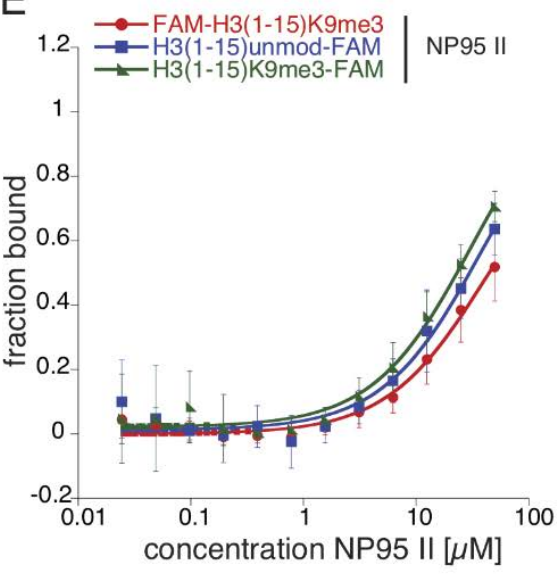

Figure 3-8 Binding behavior of the two NP95 isoforms

(A) Schematic representation of the domain organization of UHRF1, NP95 I and NP95 II. The TTD-PHD linker and the PBR region (red) and the sequence of the TTD-PHD linker insertion of NP95 I are highlighted. (B) Full length NP95 I and NP95 II wild type (NP95 FL) were used in a peptide pull down assay with no peptide (mock) or biotin conjugated H3 unmodified (H3 unmod) and H3K9me3 1-20 (H3K9me3) peptides. Western blot analysis of material recovered after washing is shown. (C) UHRF1, (D) NP95 I and (E) NP95 II full-length wild type protein was used in fluorescence polarization experiments with the indicated peptides. Fraction bound of labeled peptides was plotted depending on protein concentration. Error bars represent standard deviation of at least three independent experiments. 


\subsubsection{Mutation of the PBR in NP95 affects binding to the $\mathrm{H} 3$ tail}

Based on my previous results, I deduced a role of the PBR region in the regulation of H3K9me3 binding of NP95 I but not NP95 II. Thus, I wanted to analyze the role of the PBR in the NP95 proteins. In UHRF1, we had shown that mutation of basic residues in the PBR (K644A, K6464, K648A, R649A, K650A, S651A) released the block of the TTD domain, which resulted in improved binding to $\mathrm{H} 3 \mathrm{~K} 9 \mathrm{me} 3$. Therefore, I wanted to investigate the influence of similar mutations on the H3 tail binding of NP95 I and II.

The sequence comparison of the PBR region in the human and murine proteins had shown high sequence similarity and a common K-G-K-X-K-X-K-S motif. Based on this consensus motif, I generated a first PBR mutant in NP95 (4K-A). Studying the sequence of the SRA-RING linker in NP95, I found a second basic patch with the sequence K-E-K-S-RK-R. Since this sequence had considerable similarities with the consensus motif, I decided to generate a second PBR mutant in NP95 (3K-A). I performed mutagenesis of lysine residues in both these regions to impair charge-based interactions of these polybasic patches. In the first mutant, I replaced the K622, K624, K627 in NP95 I or K614, K616, K619 in NP95 II with alanine (3K-A). In the second mutant, I mutated K641, K644, K646, K648 in NP95 I or K633, K636, K638, K640 in NP95 II to alanine (4K-A; Figure 3-9A).

Fluorescence polarization measurements of these mutants showed that the 3K-A mutation caused a moderate loss of binding to all C-terminally H3(1-15)K9me3-FAM peptide in context of NP95 I $(\mathrm{Kd}=23.7 \mu \mathrm{M})$ as well as NP95 II $(\mathrm{Kd}=43.9 \mu \mathrm{M})$ (Figure 3-9B, C). In NP95 II the mutation additionally caused a severe loss of FAM-H3(1-15)K9me3 peptide interaction (Figure 3-9C). Comparing NP95 I and NP95 II 3K-A mutants I recognized that the mutation had similar effects on the binding behavior of both proteins.

As described, the $4 \mathrm{~K}-\mathrm{A}$ mutations comprised residues of the conserved PBR consensus sequence (Figure 3-7C). The NP95 I 4K-A mutant exhibited comparable binding behavior as the wild type protein (Figure 3-9D, 3-8D). In contrast, in NP95 II the 4K-A mutation led to a moderate loss of binding to H3(1-15)K9me3-FAM and H3(1-15)unmod-FAM peptides and had stronger negative effect on binding to the FAM-H3(1-15)K9me3 peptide (Figure 3-9E). 

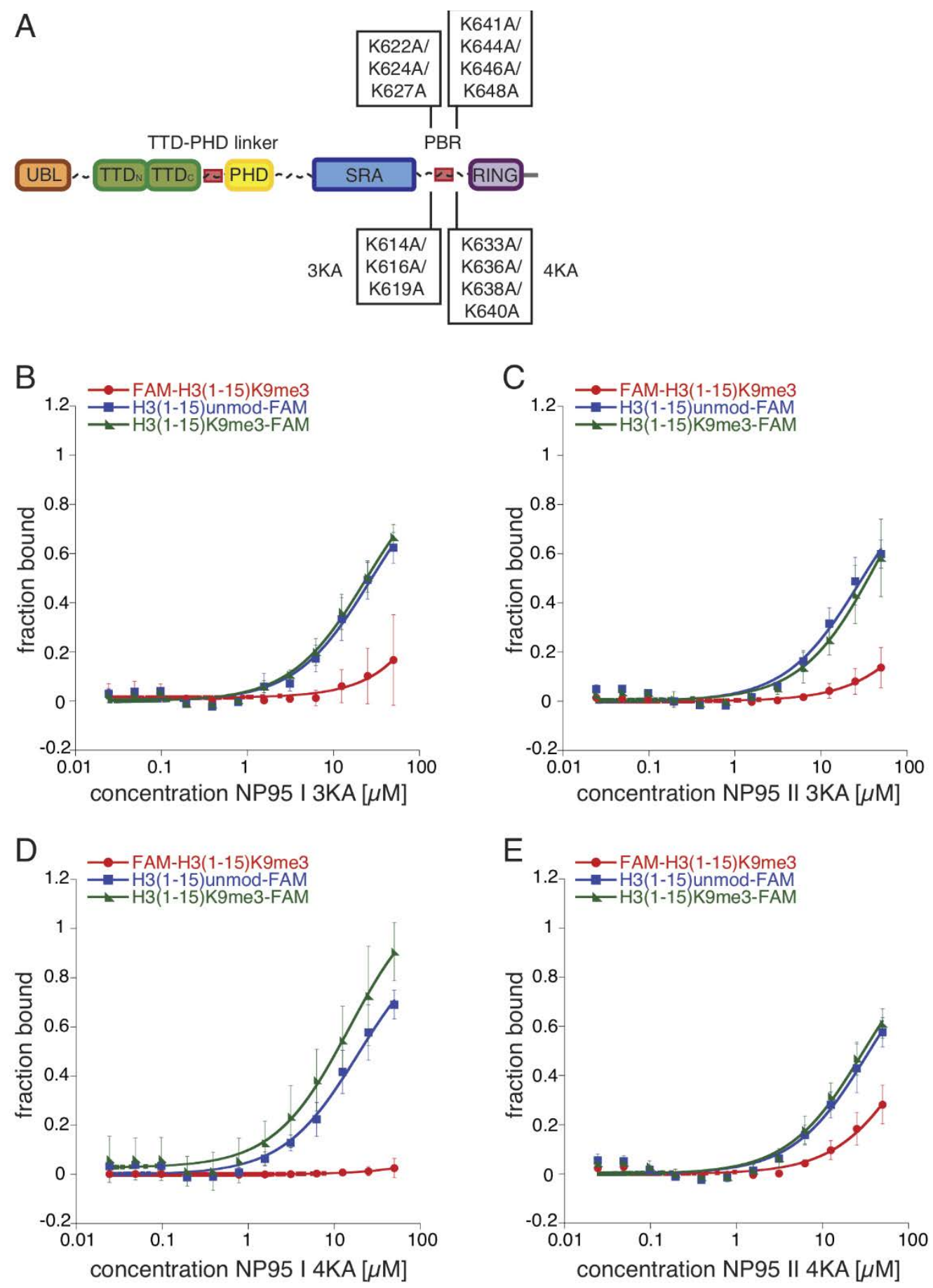

Figure 3-9 PBR mutations of NP95 I and II affect the binding to the H3 tail

(A) Schematic representation of the domain architecture of NP95. Mutants used for the experiments are indicated. (B) NP95 I K622A, K624A, K627A mutant (3KA mt) and (C) NP95 II K614A, K616A, K619A mutant (3KA $\mathrm{mt}$ ) were used in fluorescence polarization binding experiments with indicated FAM-labeled peptides. (D) NP95 I K641A, K644A, K646A, K648A mutant (4KA mt) and (E) NP95 II K633A, K636A, K638A, K640A mutant (4KA $\mathrm{mt}$ ) were used in fluorescence polarization binding experiments with indicated FAM-labeled peptides. Fraction bound of labeled peptides was plotted depending on protein concentration. Error bars represent standard deviation of at least three independent experiments

NP95 II binding to the H3 tail was negatively affected by the 3K-A and 4K-A mutations. As NP95 II, NP95 I binding affinity for H3 tail peptides was decreased by the 3K-A mutation. However no effect on the binding was detected upon mutation of the common 
K-G-K-X-K-X-K-S motif (4K-A). In contrast to UHRF1, mutations of polybasic patches in the SRA-RING linker had a different effect on the H3 tail binding behavior of NP95. In conclusion, the PBR regions of NP95 I and II have functions different from the UHRF1 PBR.

\subsubsection{The PBR region does not affect TTD binding to H3K9me3 in NP95}

We had shown that the PBR in UHRF1 interacts with the TTD domain. This interaction is the basis of the PBR dependent regulatory mechanism. Based on my former results, I deduced a different role of the PBR region in UHRF1 and NP95. To analyze these differences between UHRF1 and NP95 in greater detail, I wanted to investigate differences in the PBR and TTD interaction of the proteins as well as the influence of the PBR on the binding of the TTD.

Initially, I investigated the interaction between the two TTD-PHD cassettes and the PBRRING of UHRF1 and NP95 using co-immunoprecipitation. I used a specific antibody to immobilize UHRF1 PBR-RING on beads. After incubation with TTD-PHD in a 1:1 molar ratio and washing, I detected strong recovery of TTD-PHD on PBR-RING coupled beads but not on antibody control beads. This result showed a strong interaction of these two regions in UHRF1 (Figure 3-10A). I performed a similar experiment with immobilized PBR-RING and the two TTD-PHD regions of NP95 using a 5-fold molar excess of the TTD-PHD (Figure 3-10B). This experiment showed a weak interaction of the PBR-RING with TTD-PHD of NP95 I but not NP95 II This weak interaction raised questions if the PBR region of NP95 could influence the binding of the TTD to H3K9me3.

Next, I wanted to determine if the PBR-RING causes the blocking of the TTD domain and a loss of binding to H3K9me3 in NP95 I as it was shown for UHRF1 (Gelato et al., 2014). I used fluorescence polarization to characterize changes in binding of the TTD of NP95 to H3K9me3 upon addition of PBR-RING. I performed similar experiments with UHRF1 as a control. The UHRF1 TTD domain alone was specifically binding to H3K9me3 peptides, while it did not interact with the unmodified peptide (Figure 3-10C). As already described, the binding to $\mathrm{H} 3 \mathrm{~K} 9 \mathrm{me} 3$ peptide was lost upon addition of 2-fold molar excess of PBR-RING to the binding reaction (Figure 3-10D) (Gelato et al., 2014). The TTD domain of NP95 showed specific binding to the FAM-H3(1-15)K9me3 and H3(1-15)K9me3-FAM peptides, comparable with the UHRF1 TTD (Figure 3-10C, E). In contrast to UHRF1, the NP95 TTD did not exhibit any significant change in its binding to FAM-H3(1-15)K9me3 peptide in presence of 2-fold molar excess of PBR-RING (Figure 3-16C, D). Contrary, there was substantial gain of binding of both TTD-PHD to the H3(1-15)unmod-FAM when PBRRING was added (Figure 3-10D, F). 


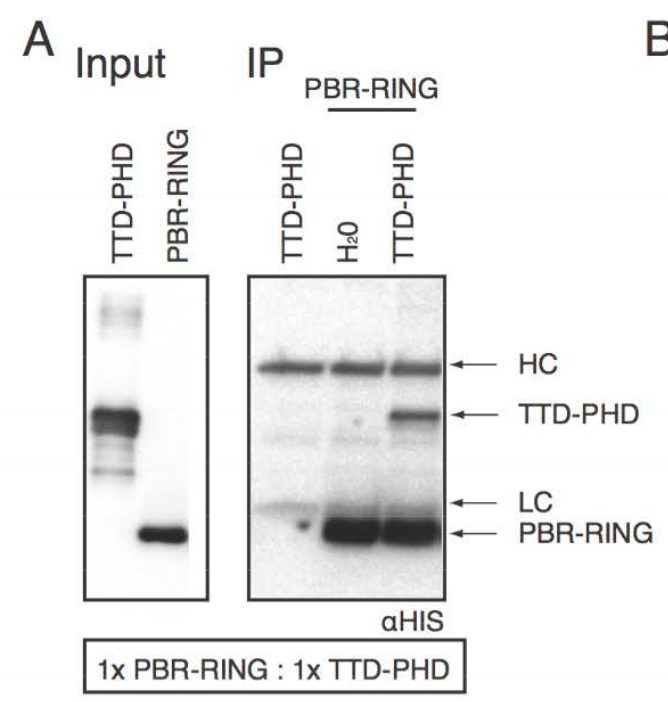

B
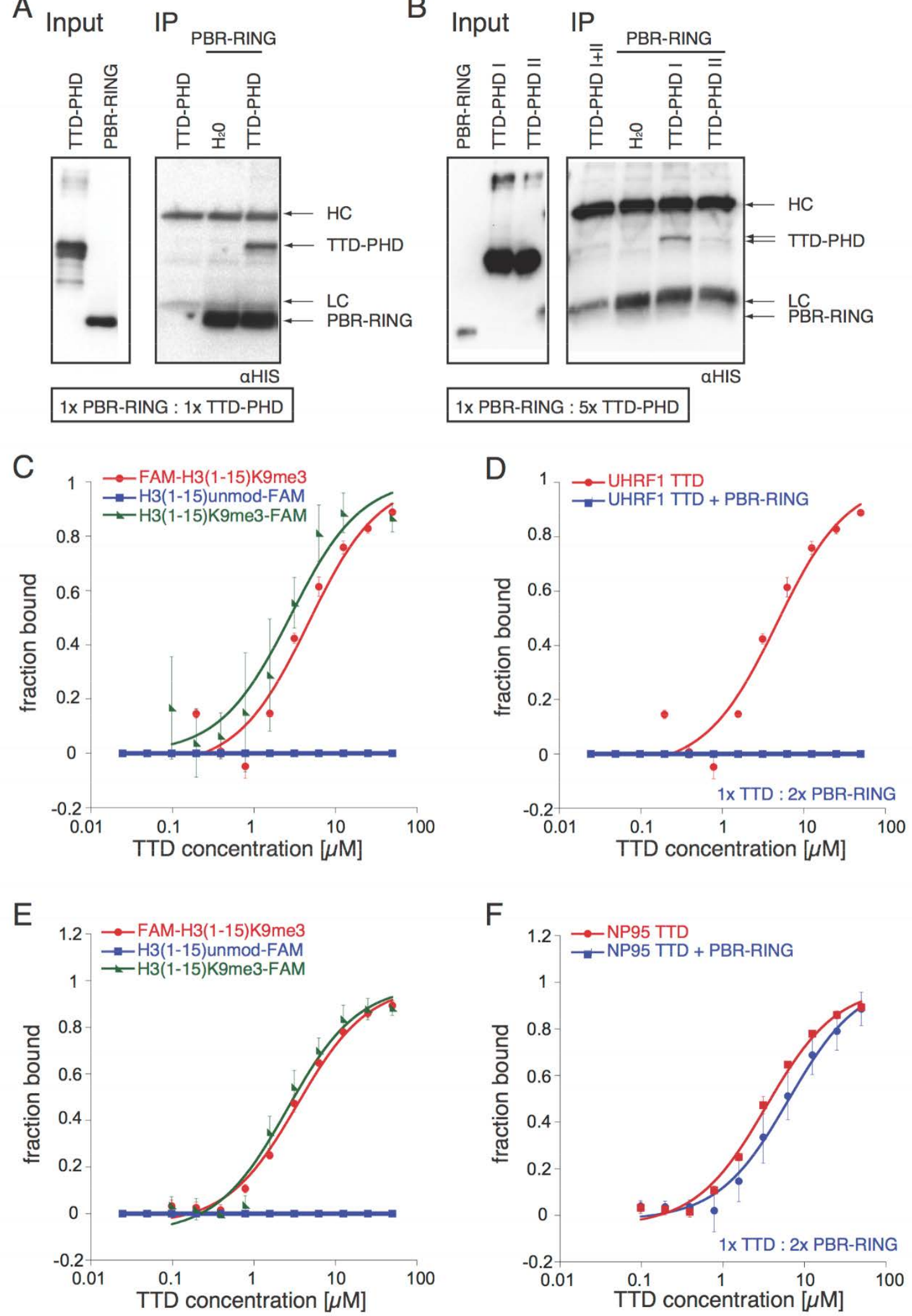

Figure 3-10 Weak interaction of the TTD-PHD and PBR-RING region of NP95

(A) UHRF1 PBR-RING region was coupled to beads using a specific antibody. Beads conjugated with the PBR-RING or control beads were then incubated with UHRF1 TTD-PHD (Input) and used in a co-immunoprecipitation assay (IP). The material retained after washing was analyzed by western blot using $\alpha$-His antibody. (B) NP95 PBR-RING region was coupled to beads using a specific antibody. Beads conjugated with the PBR-RING or control beads were then incubated with NP95 I or II TTD-PHD (Input) and used in a co-immunoprecipitation assay (IP). The material retained after washing was analyzed by western blot using 
$\alpha$-His antibody. (C) Fluorescence polarization binding experiments of UHRF1 TTD interaction with indicated FAM-labeled peptides. (D) Fluorescence polarization binding experiments of UHRF1 TTD interaction with FAM-H3(1-15)K9me3 peptide with and without 2-fold excess of PBR-RING added. (E) Fluorescence polarization binding experiments of NP95 TTD interaction with indicated FAM-labeled peptides. (F) Fluorescence polarization binding experiments of UHRF1 TTD interaction with FAM-H3(1-15)K9me3 peptide with and without 2-fold excess of PBR-RING added. Fraction bound of labeled peptides was plotted depending on protein concentration. Error bars represent standard deviation of at least three independent experiments.

Contrary to the effects seen in context of UHRF1, my results showed that the NP95 PBR did not interact with the TTD-PHD region of NP95 II but was weakly binding to TTDPHD of NP95 I. My previous results showed that the TTD domain of NP95 I was blocked in context of the full-length protein. However, I could not detect an effect of the PBR on the target binding of the NP95 TTD, as detected for UHRF1 TTD (Gelato et al., 2014). This revealed major differences in the regulation of NP95 I and UHRF1 TTD interaction with H3K9me3. Further, these findings suggested that the TTD in context of NP95 I is blocked by a different region but the PBR.

\subsubsection{NP95 binding to $\mathrm{H} 3$ tail is regulated by different TTD-PHD linker regions}

\subsubsection{The TTD-PHD region of UHRF1 and NP95 II shows similar H3 tail binding}

Based on the different H3 tail binding behavior of NP95 I and II in context of the full length protein, I hypothesized that the TTD blocking observed in NP95 I might be a result of the TTD-PHD linker insertion. To investigate the role of the TTD-PHD linker in the NP95 isoforms in comparison to UHRF1, I generated TTD-PHD fragments of UHRF1 and the NP95 variants (Figure 3-11A). I performed a peptide pull down assay, using peptides comprising amino acids 1 to 20 of the $\mathrm{H} 3$ tail unmodified as well as tri-methylated at $\mathrm{K} 9$. $\mathrm{H} 3(1-20)$ unmodified or $\mathrm{H} 3(1-20) \mathrm{K} 9 \mathrm{me} 3$ peptide were C-terminally biotin-labeled. Our laboratory had previously shown that UHRF1 TTD-PHD specifically enriches at H3(120)K9me3 peptide in this context (Gelato et al., 2014). I analyzed the TTD-PHD of the two NP95 isoforms in the same way. The results showed that NP95 II TTD-PHD preferentially bound to the H3K9me3 peptide, comparable to UHRF1 (Figure 3-11B). In contrast, I found that TTD-PHD of NP95 I was equally recovered by the H3(1-20) unmodified and H3(120)K9me3 peptides. 
A

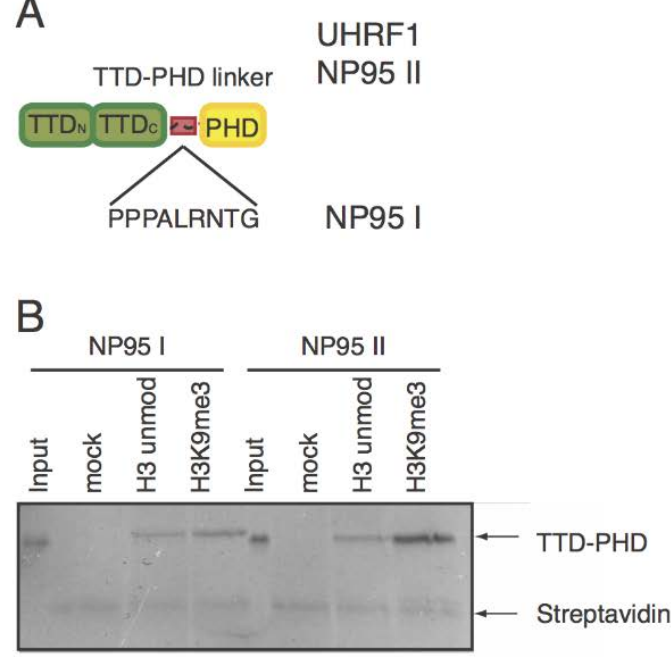

D

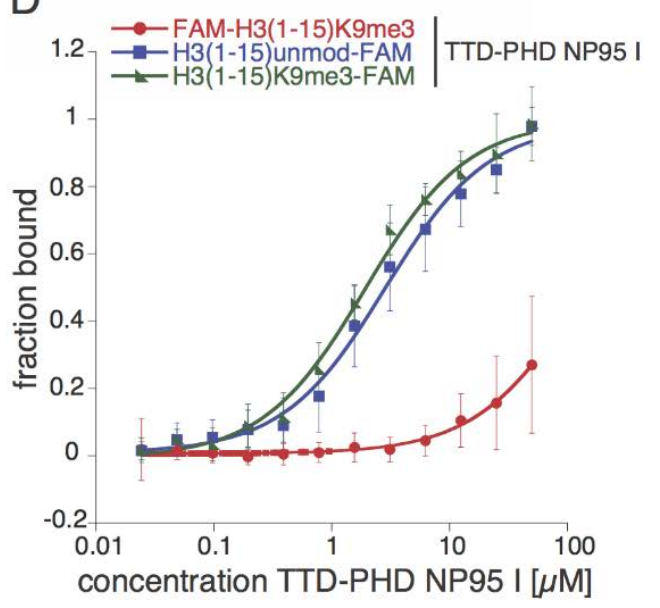

C

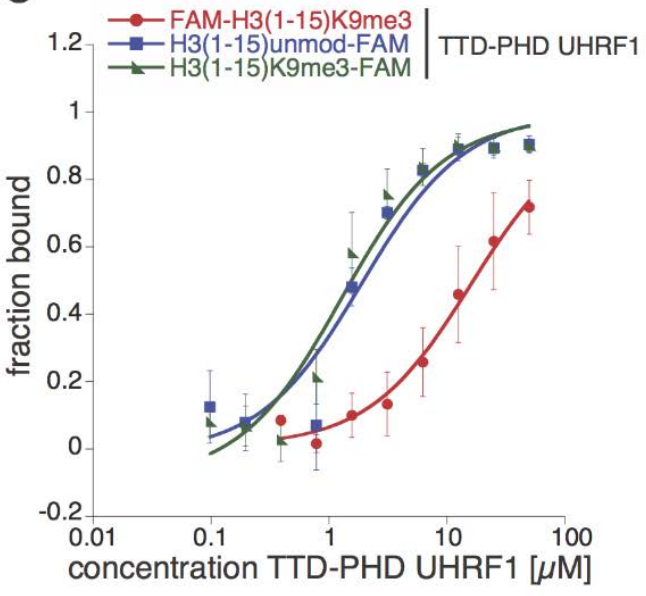

$\mathrm{E}$

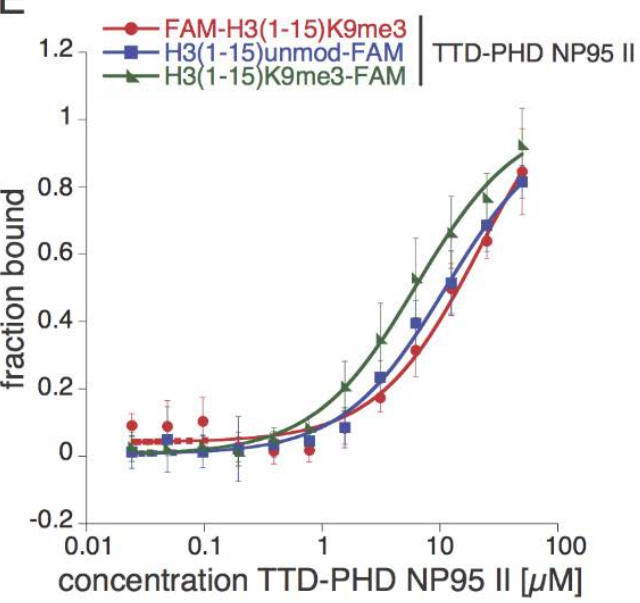

Figure 3-11 Binding behavior of the TTD-PHD fragments of the two NP95 isoforms

(A) Schematic representation of the TTD-PHD fragment of UHRF1, NP95 I and NP95 II the TTD-PHD linker (red) and the sequence of the TTD-PHD linker insertion of NP95 I are highlighted. (B) TTD-PHD fragment of NP95 I and NP95 II wild type (TTD-PHD) were used in a peptide pull down assay with no peptide (mock) or biotin conjugated $\mathrm{H} 3(1-20)$ unmodified (H3 unmod) and $\mathrm{H} 3(1-20) \mathrm{K} 9 \mathrm{me} 3$ (H3K9me3) peptides. Material recovered after washing was analyzed by western blot. (C) UHRF1, (D) NP95 I and (E) NP95 II wild type TTD-PHD fragments were used in fluorescence polarization experiments with the indicated peptides. Fraction bound of labeled peptides was plotted depending on protein concentration. Error bars represent standard deviation of at least three independent experiments.

Furter, I analyzed the TTD-PHD domains in fluorescence polarization binding experiments using the described fluorescently labeled peptides. The binding studies of UHRF1 TTD-PHD verified the described differences compared to the full-length protein (Gelato et al., 2014). The protein bound to all peptides analyzed with a strong preference for C-terminally FAM labeled peptides (Figure 3-11C). In contrast, the NP95 I TTD-PHD did not bind to the FAM H3K9me3 1-15 peptide. However, it showed robust interaction with the H3(1-15)unmod-FAM $(\mathrm{Kd}=2.9 \mu \mathrm{M})$ and H3(1-15)K9me3-FAM $(\mathrm{Kd}=1.9 \mu \mathrm{M})$ 
peptides (Figure 3-11D). NP95 II TTD-PHD interacted with FAM-H3(1-15)K9me3 (Kd = 22 $\mu \mathrm{M})$ and $\mathrm{H} 3(1-15)$ unmod-FAM $(\mathrm{Kd}=11 \mu \mathrm{M})$. The interaction with the bivalent peptide, $\mathrm{H} 3(1-15) \mathrm{K} 9 \mathrm{me} 3-\mathrm{FAM}(\mathrm{Kd}=6 \mu \mathrm{M})$, was stronger compared to the peptides that were only bound by TTD or PHD domain alone (Figure 3-11E).

In agreement with the results of the peptide pull down assay (Figure 3-11B), the TTDPHD of NP95 II bound to H3 peptides comparable to UHRF1 (Figure 3-11C, E). It interacted with all peptides independent of their modification status, suggesting a contribution of TTD as well as PHD to H3 tail interaction. In contrast, NP95 I TTD-PHD did not interact with the FAM-H3(1-15)K9me3 peptide that exclusively binds to the TTD domain. I reasoned that the TTD domain is not able to bind to K9me3 in context of the NP95 I TTD-PHD. Given the differences in binding of NP95 I and NP95 II TTD-PHD to H3K9me3, the results suggested a potential role of the NP95 I TTD-PHD linker insertion in blocking the TTD/H3K9me3 interaction.

\subsubsection{The TTD domain is blocked in NP95 I but not NP95 II}

The results to this end indicated that the TTD domain of NP95 I is not binding to H3K9me3 in context of the isolated TTD-PHD region and the full-length protein, while the TTD of NP95 II is functional under comparable conditions. To validate these differences in the $\mathrm{H} 3$ tail binding of NP95 I and II, I investigated the respective TTD and PHD mutants of both proteins (Figure 3-12A). I prepared mutants that perturbed the aromatic cage of the NP95 TTD (Y184A, Y187A) or the interaction of PHD with the A1 and R2 of the H3 tail (D339A, E340A or D331A, E332A) based on sequence conservation and domain similarity with UHRF1 (Gelato et al., 2014; Rothbart et al., 2013). I analyzed the resulting mutant TTD-PHD fragments of NP95 I and NP95 II using fluorescence polarization binding experiments.

I found that the mutation of the TTD (Y184A, Y187A) of NP95 I TTD-PHD did not change the binding behavior compared to the wild type TTD-PHD (Figure 3-12B). The protein was still binding the C-terminally FAM-labeled H3(1-15)unmod $(\mathrm{Kd}=10.3 \mu \mathrm{M})$ and H3(1-15)K9me3 $(\mathrm{Kd}=12.1 \mu \mathrm{M})$ peptides but not the N-terminally FAM-labeled H3(1-15)K9me3 peptide. At the same time, the mutation of the NP95 I PHD domain (D339A, E340A) led to a severe loss of binding to all of three tested peptides (Figure 312C). In contrast, the TTD mutant of NP95 II (Y184A, Y187A) still bound to the two

C-terminally labeled peptides $(\mathrm{Kd}=10.4 \mu \mathrm{M} ; \mathrm{Kd}=13.6 \mu \mathrm{M})$ but not to the N-terminally FAM-labeled H3(1-15)K9me3 (Figure 3-12D). The NP95 II PHD mutant (D331A, E332A) interacted with the N-terminally and C-terminally FAM-labeled H3(1-15)K9me3 peptides 
$(\mathrm{Kd}=16 \mu \mathrm{M} ; \mathrm{Kd}=28 \mu \mathrm{M})$, while the H3(1-15)unmod-FAM peptide was not engaged (Figure 3-12E).
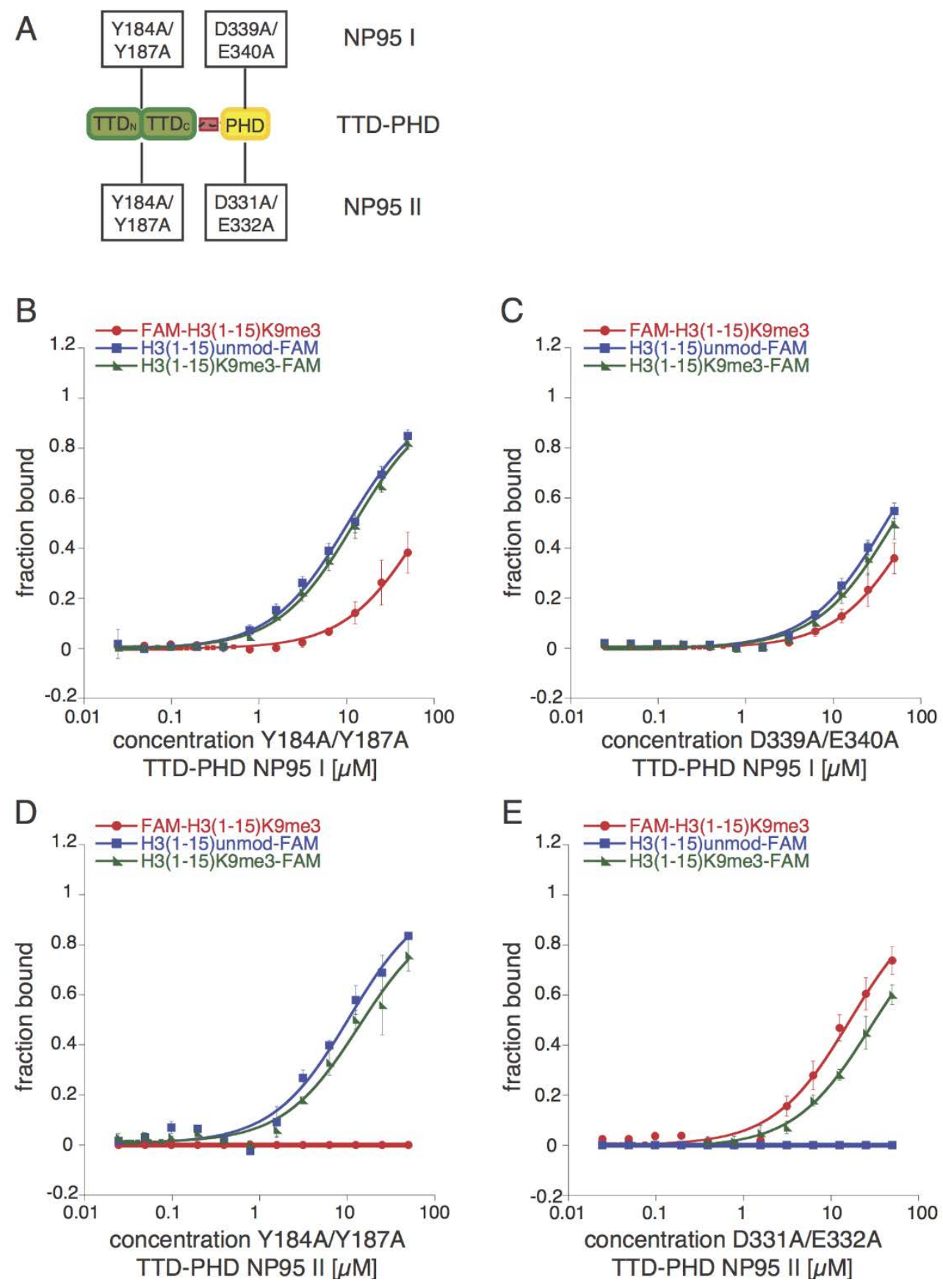

Figure 3-12 Differential H3 tail binding by PHD mutants of the two NP95 TTD-PHD fragments

(A) Schematic representation of the TTD-PHD fragment of NP95 I and NP95 II the TTD-PHD linker (red) and the TTD-PHD fragment mutants that were used for the experiments are highlighted. NP95 I TTD-PHD fragment TTD mutant (Y184A, Y187A) (B) and PHD mutant (D339A E340A) (C) were used in fluorescence polarization experiments with the indicated peptides. NP95 II TTD-PHD fragment TTD mutant (Y184A, Y187A) (D) and PHD mutant (D339A, E340A) (E) were used in fluorescence polarization experiments with the indicated peptides. Fraction bound of labeled peptides was plotted depending on protein concentration. Error bars represent standard deviation of at least three independent experiments. 
Since mutation of the PHD domain had different consequences in NP95 I and II TTD-PHD fragments, I concluded that NP95 I TTD-PHD establishes interaction with the H3 tail only by the PHD domain. The mutation of the PHD domain of NP95 I caused a total loss of interaction with the H3 tail, whereas mutation of the PHD domain of NP95 II only led to loss of binding to the H3(1-15)unmod-FAM peptide. This suggested that the NP95 I TTD domain interaction with H3K9me3 is blocked. Since both NP95 wild type TTD-PHD fragments only differed by a nine amino acid insertion in the TTD-PHD linker, I reasoned that this linker region is directly or indirectly causing the block of the TTD.

\subsubsection{The NP95 I TTD is blocked by the TTD-PHD linker insertion}

Based on my previous results, I hypothesized that the TTD-PHD linker insertion of NP95 I is responsible for the blocking of the TTD/H3K9me3 interaction. Therefore, I investigated the effect of different mutations in the TTD-PHD linker insertion of NP95 I on the TTD binding to H3K9me3 (Figure 3-13A). In addition to that, I analyzed the PHD mediated interaction with the unmodified N-terminus of $\mathrm{H} 3$. Considering the sequence of the TTD-PHD linker insertion (P-P-P-A-L-R-N-T-G), I decided to mutate the proline patch (P293A, P294A, P295A). I rationalized that the conformational rigidity of proline affects the relative spacing of TTD and PHD as well as the positioning of the linker on the TTD surface. Given the importance of basic residues in general and the R-K-S sequence motif in particular for the interaction of TTD with different linker regions, I also mutated an arginine in the center of the linker (R298A) as well as a lysine and a serine (K302A, S303A) adjacent to the TTD-PHD linker insertion (Arita et al., 2012; Rothbart et al., 2013). I used fluorescence polarization measurements to investigate the $\mathrm{H} 3$ tail binding of these mutants.

The NP95 I TTD-PHD PPP-AAA (P293A, P294A, P295A) $(\mathrm{Kd}=11.1 \mu \mathrm{M})$ and R-A (R298A) $(\mathrm{Kd}=5.0 \mu \mathrm{M})$ mutants showed a significant gain of binding to H3K9me3 peptide compared to the wild type TTD-PHD region (Figure 3-13B). At the same time, the KS-AA (K302A, S303A) mutant did not interact with the FAM-H3K9me3 peptide. Additional measurements with the described TTD-PHD mutants revealed no change of binding affinity to H3(1-15)unmod-FAM compared to the wild type TTD-PHD region (Figure 3-13C). Subsequent analysis of the full-length NP95 I PPP-AAA and R-A mutants did not resemble the effects that were detected in contexts of the TTD-PHD fragment. The binding of the two mutant proteins to FAM-H3K9me3 peptide did not differ from the wild type and KS-AA mutant (Figure 3-13D). The interaction of the full-length NP95 I proteins with the H3(1-15)unmod-FAM was not affected by any of the TTD-PHD linker mutations (Figure 3-11E). 
A

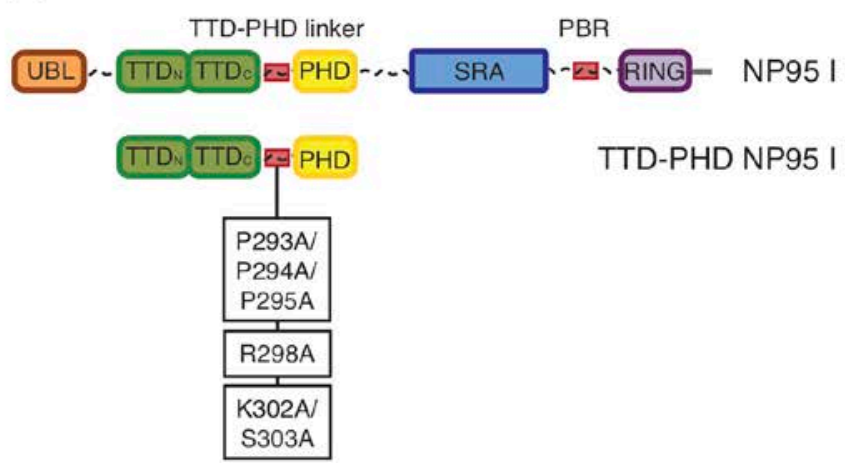

$\mathrm{B}$

C
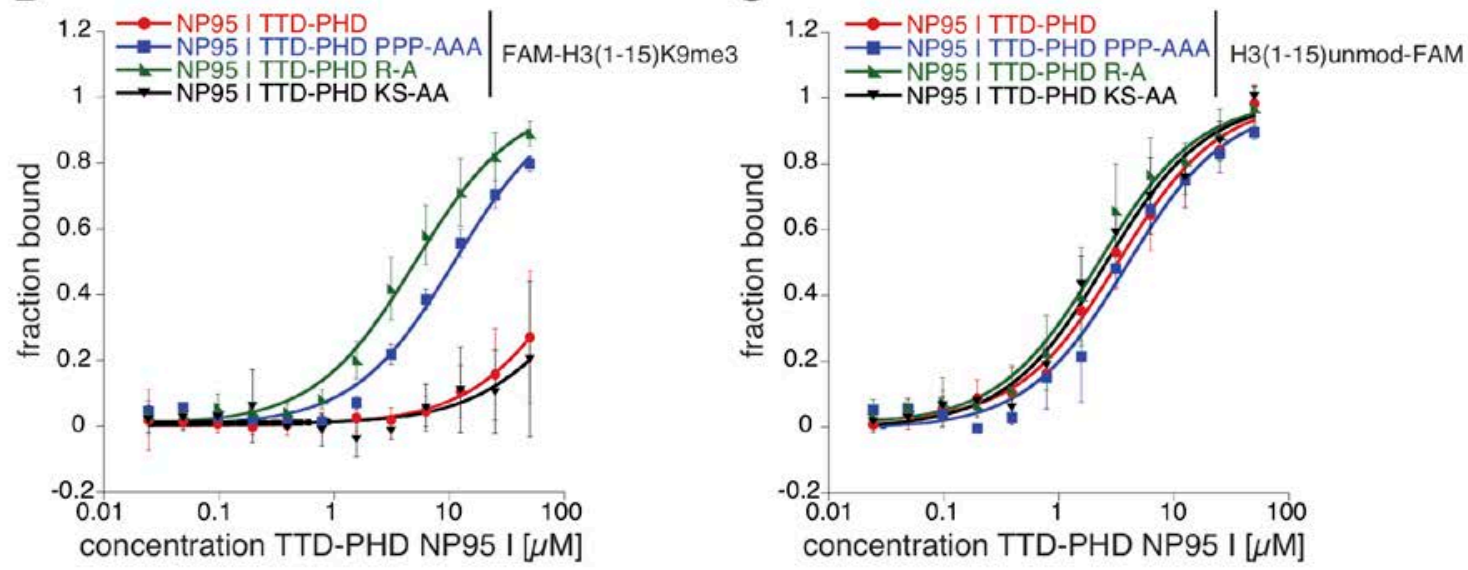

D

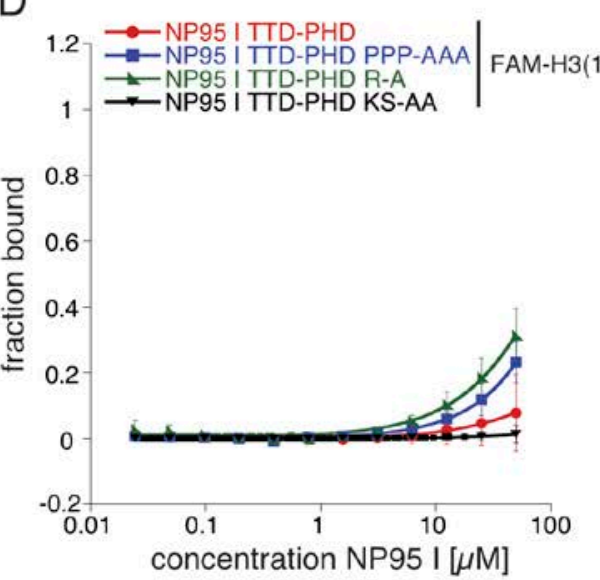

$\mathrm{E}$

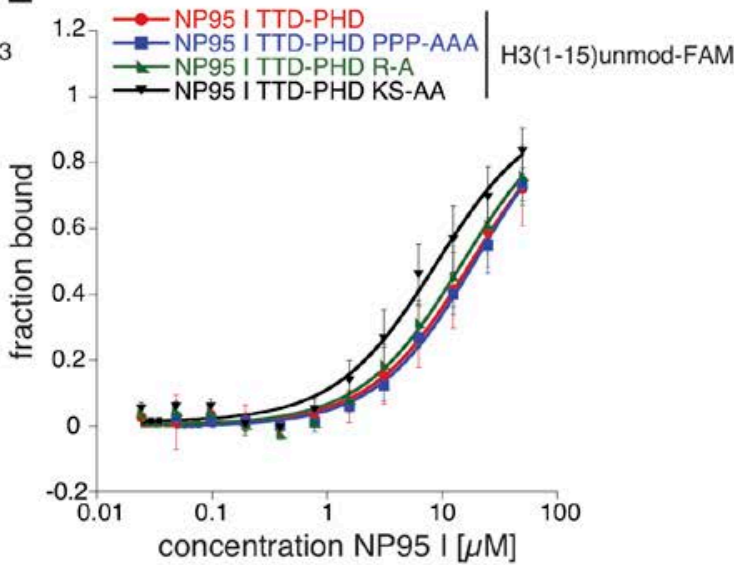

Figure 3-13 Binding behavior of TTD-PHD linker mutants of NP95 I TTD-PHD fragment

(A) Schematic representation of the full-length protein and TTD-PHD fragment of NP95 I the TTD-PHD linker and the PBR region (red) and TTD-PHD linker mutants that were used for the experiments are highlighted. (B, C) TTD-PHD fragment of wild type and different TTD-PHD linker mutants of NP95 I (P293A, P294A, P295A; R298A; K302A, S303A) were used in fluorescence polarization experiments with the indicated peptides. (D, E) Wild type and different TTD-PHD linker mutants of full-length NP95 I (P293A, P294A, P295A; R298A; K302A, S303A) were used in fluorescence polarization experiments with the indicated peptides. Fraction bound of labeled peptides was plotted depending on protein concentration. Error bars represent standard deviation of at least three independent experiments. 
Altogether, these results indicated an important contribution of P293, P294, P295 and R298A residues but not K302A, S303A to TTD blocking in NP95 I TTD-PHD. Since the PHD domain binding to the unmodified $\mathrm{N}$-terminus of the $\mathrm{H} 3$ tail did not change upon mutation of the linker sequence, I concluded that the linker is exclusively affecting the TTD. At the same time, the mutations had minor influence on the $\mathrm{H} 3$ tail binding behavior of the full-length NP95 I. I reasoned that in context of the full-length protein TTD binding regulation might be more complex and secondary interaction might stabilize the blocking by the TTD-PHD linker insertion.

\subsubsection{The NP95 I TTD-PHD linker insertion changes UHRF1 binding behavior}

It was reported before that transfer of the whole TTD-PHD linker of NP95 I to the structurally related NP97/NIRF (mUHRF2) causes blocking of H3K9me3 binding in this protein (Pichler et al., 2011). I wanted to investigate if the 9 amino acid NP95 I linker insertion alone is sufficient to cause blocking of the UHRF1 TTD domain. In addition to that, I wanted to analyze if the presence of the NP95 I TTD-PHD linker in the context of full-length UHRF1 is affecting the TTD/ PBR interaction. To this end, I inserted the 9 amino acids into the TTD-PHD linker of UHRF1 TTD-PHD and full-length protein in a way that disrupted the R-K-S motif for TTD interaction ( $\underline{R} \underline{R S} \rightarrow \underline{R-P P P A L R N T G-K S}$ ) (Figure 3-14A). I determined the binding affinities of the UHRF1 TTD-PHD fragment and full-length protein carrying this mutation in fluorescence polarization binding experiments.

I observed that the full-length UHRF1 mutant bound to H3(1-15)unmod-FAM $(\mathrm{Kd}=13.9 \mu \mathrm{M})$ and $\mathrm{H3}(1-15) \mathrm{K} 9 \mathrm{me3}-\mathrm{FAM}(\mathrm{Kd}=16.9 \mu \mathrm{M})$, while the N-terminally FAMlabeled H3(1-15)K9me3 remained unbound (Figure 3-12B). Therefore, the binding behavior of the mutant did not change compared to the wild type UHRF1 (Figure 3-8C). The UHRF1 TTD-PHD NP95 I insertion mutant showed strong binding to the Cterminally labeled peptides and only weak interaction with the FAM-H3K9me3 1-15 $(\mathrm{Kd}=64.9 \mu \mathrm{M})$ (Figure 3-14C). Compared to the wild type UHRF1 TTD-PHD, the mutant did exhibit a substantial change in binding behavior, in which the interaction with FAMH3K9me3 was 4-fold reduced (Figure 3-11C). These results suggested that blocking of TTD binding to H3K9me3 by the NP95 I TTD-PHD linker insertion was also established in this context. The addition of UHRF1 PBR-RING to the fluorescence polarization measurement of the UHRF1 TTD-PHD insertion mutant led to an additional loss of binding to N-terminally labeled H3K9me3 peptide (Figure 3-14D). 
A

insertion mutant

TTD-PHD linker

PBR

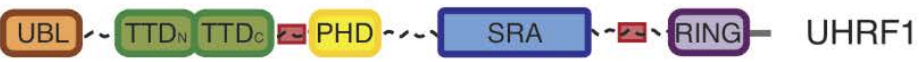

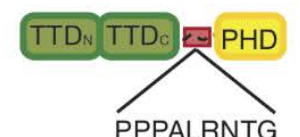

PPPALRNTG

NP95 I TTD-PHD linker insertion

B

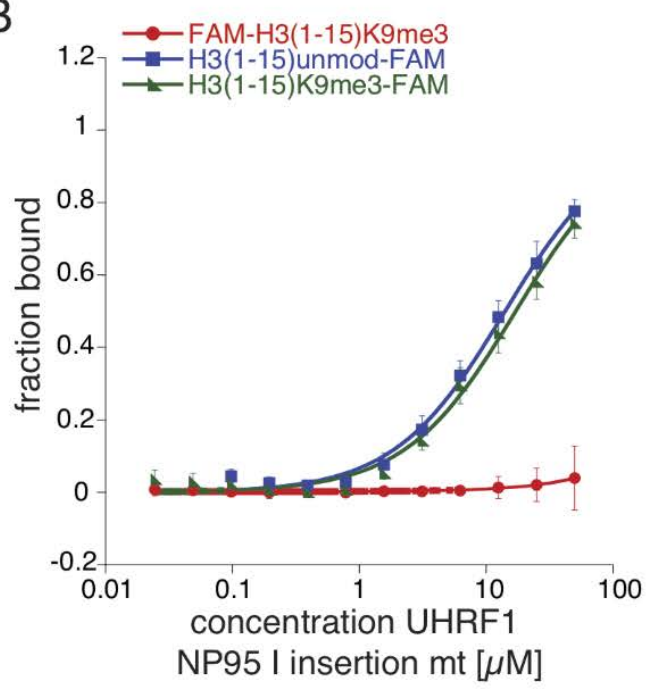

D

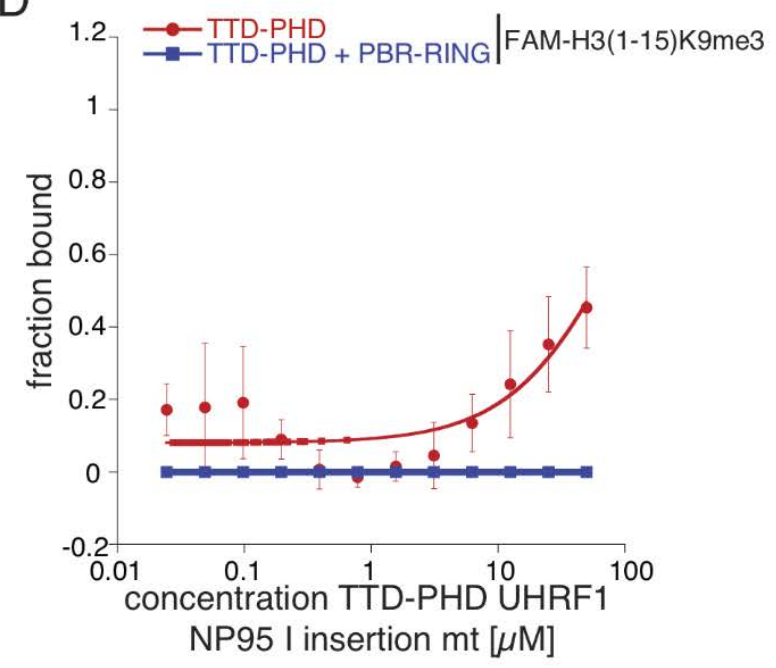

Figure 3-14 NP95 I TTD-PHD linker insertion reduced binding of UHRF1 TTD-PHD to H3K9me3

(A) Schematic representation of the full-length protein and TTD-PHD fragment of UHRF1. The TTD-PHD linker and the PBR region (red) and the TTD-PHD linker mutant with the NP95 I insertion used for the experiments are highlighted. Full-length UHRF1 (B) and TTD-PHD fragment (C) of NP95 I TTD-PHD linker insertion mutant protein were analyzed in fluorescence polarization experiments with the indicated peptides. (D) Fluorescence polarization binding experiments of the interaction TTD-PHD fragment of NP95 I TTD-PHD linker insertion mutant of UHRF1 with FAM-H3(1-15)K9me3 peptide with and without 2-fold molar excess of UHRF1 PBR-RING fragment added. Fraction bound of labeled peptides was plotted depending on protein concentration. Error bars represent standard deviation of at least three independent experiments. 
The results showed that the NP95 I linker insertion causes reduced TTD interaction with H3K9me3 in context of UHRF1 TTD-PHD. Since addition of UHRF1 PBR-RING led to further reduction of TTD interaction with $\mathrm{H} 3 \mathrm{~K} 9 \mathrm{me} 3$, I reasoned that both linker regions make contacts with the TTD. In addition to that, my results showed stronger interaction between TTD and PBR region compared to TTD and the TTD-PHD linker insertion. Since I detected strong blocking of the TTD by the TTD-PHD linker in context of NP95 I, I concluded that the different blocking mechanisms might use specific surfaces on the TTD of both proteins. These surfaces differ between UHRF1 and NP95 and, thus, cause the observed differences. Therefore, the results suggest that TTD surface is adapted to the interaction with the PBR in UHRF1 and the TTD-PHD linker in NP95.

\subsubsection{An R-K-S motif in the TTD-PHD linker influences binding of NP95 II to the $\mathrm{H} 3$ tail}

It has been suggested that the UHRF1 TTD-PHD linker establishes a multivalent binding state of TTD and PHD (Arita et al., 2012; Rothbart et al., 2013). An R-K-S sequence motif in the linker plays an essential role in this process. Three TTD surface residues, G236, F237 and W238 were found to be essential for the interaction with the TTD-PHD linker RKS motif (Arita et al., 2012; Cheng et al., 2013; Rothbart et al., 2013; Xie et al., 2012).

I used the PRALINE sequence alignment tool to examine sequence conservation of these residues in NP95 (http://zeus.few.vu.nl/programs/pralinewww/). I found that the essential residues G236, F237 and W238 were conserved in the murine proteins (Figure 315A). I reasoned that the reported function of the R-K-S motif might be conserved as well. To this end, I mutated the TTD-PHD linker R-K-S sequence motif (R293A, K294A, S295A) in context of the NP95 II TTD-PHD to impair the putative TTD/TTD-PHD linker interaction (Figure 3-15B).

I analyzed the influence of this motif on the individual binding behavior of the TTD and PHD by fluorescence polarization measurements using the FAM-H3(1-15)K9me3 and the H3(1-15)unmod-FAM peptides. In addition to that, I investigated the multivalent binding of TTD and PHD domains to the same H3 tail using the C-terminally FAM-tagged H3(1-15)K9me3 peptide.

The mutation of the R-K-S motif in NP95 II TTD-PHD caused a minor loss of binding to the FAM-H3(1-15)K9me3 peptide $(\mathrm{Kd}=40.0 \mu \mathrm{M})$, which only interacts with the TTD domain (Figure 3-13C). At the same time, the RKS-AAA mutation caused a slight gain of binding to the $\mathrm{H} 3(1-15)$ unmod-FAM peptide $(\mathrm{Kd}=4.4 \mu \mathrm{M})$ compared to the wild type NP95 II TTD-PHD region (Figure 3-13D). The binding to the H3(1-15)K9me3-FAM 
peptide $(\mathrm{Kd}=1.6 \mu \mathrm{M})$, which can be bound by both, TTD and PHD, was increased 4-fold by the same mutation (Figure 3-13E).

Since the mutation exclusively affected the binding to the bivalent target peptide H3(1-15)K9me3-FAM, I reasoned that the mutation had influence on the combined read out of TTD and PHD domain. In UHRF1 TTD-PHD, a similar mutation of the R-R-K-S motif led to impaired multivalent binding to a similar peptide (Arita et al., 2012). I concluded that the R-K-S motif in NP95 II might establish a multivalent binding mode comparable to UHRF1.

A

TTD-PHD linker

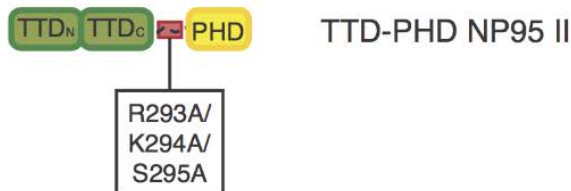

B

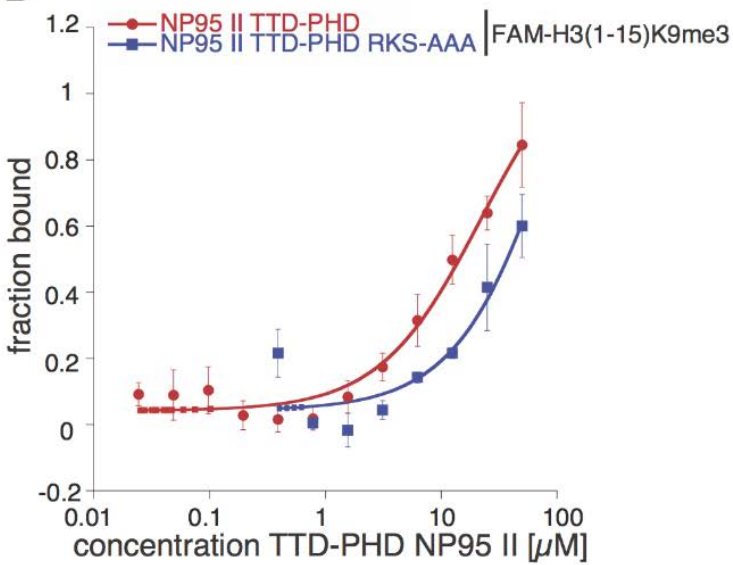

$\mathrm{D}$

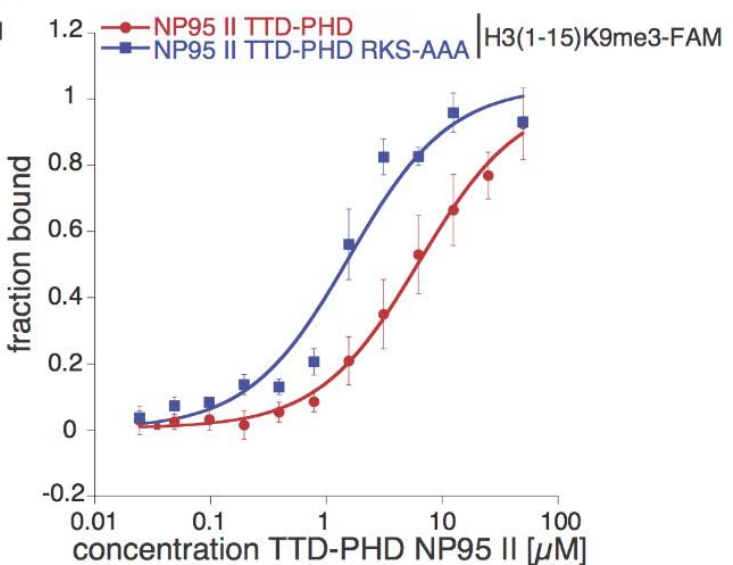

Figure 3-15 Binding behavior of TTD-PHD linker mutant of NP95 II TTD-PHD fragment

(A) Sequence alignment was prepared using the PRALINE tool (http://zeus.few.vu.nl/programs/ pralinewww/). Numbers correspond to amino acid positions at the beginning and end of the respective protein sequences of the different species. UHRF1 amino acid sequences according to NCBI: Homo sapiens, NP_001276981.1; Mus musculus V1, NP_001104550.1, Mus musculus V2, NP_001104548.1. (B) Schematic representation of the TTD-PHD fragment of NP95 II. The TTD-PHD linker (red) and TTD-PHD linker mutant used for the experiments (R293A, K294A, S295A) are highlighted. (B, C, D) TTD-PHD fragment of wild type and TTD-PHD linker mutant of NP95 II (R293A, K294A, S295A) were used in fluorescence polarization experiments with the indicated peptides. Fraction bound of labeled peptides was plotted depending on protein concentration. Error bars represent standard deviation of at least three independent experiments. 


\subsubsection{Structural differences between NP95 I and II TTD-PHD}

\subsubsection{Similar structural features of TTD-PHD region of UHRF1 and NP95 II but not NP95 I}

My previous experiments established clear differences in the function of the TTD-PHD linker in the two murine isoforms of UHRF1, NP95 I and NP95 II. The TTD-PHD linker insertion of NP95 I was shown to block the TTD domain interaction with H3K9me3. NP95 II and UHRF1 TTD-PHD linker exhibit high sequence similarity and might share the same mode of action. In collaboration with Cheryl Arrowsmith (University of Toronto, Canada) we wanted to further investigate these differences between TTD-PHD NP95 I and NP95 II. We used SAXS and NMR spectroscopy to gain further insights into structural features of TTD-PHD and the TTD-PHD linker in this context.

Small angle X-ray scattering (SAXS) and subsequent abinitio envelope prediction by DAMMIF software revealed great similarity of the overall surface shape of TTD-PHD cassettes of UHRF1 and the two murine isoforms (Figure 3-16A). We performed theoretical structure modeling on the assumption that TTD and PHD shapes were rigid while the TTD-PHD linker was flexible. This resulted in approximately 50,000 structures for TTD-PHD of NP95 I and NP95 II, respectively. These theoretical structures were plotted depending on their $\mathrm{R}_{\mathrm{g}}$ value, the mass weighted average radius (Figure 3-16B, dashed line). In conjunction with the SAXS data that was experimentally generated these theoretical structures were merged to an optimal ensemble and plotted for comparison (Figure 3-16B, solid line). We used UHRF1 TTD-PHD as reference because its structure has been studied in great detail. In UHRF1 TTD-PHD, the first peak $\left(\mathrm{R}_{\mathrm{g}}=27\right)$ corresponds to the TTD-PHD structure with the linker in the peptide binding groove. The second peak $\left(\mathrm{R}_{\mathrm{g}}=39\right)$ represented the structure where the linker is not locked on the TTD surface but is randomly moving (Arita et al., 2012). NP95 II and UHRF1 TTD-PHD optimal ensemble distribution exhibited striking similarities (Figure 3-16B, solid line). The analogous distribution of the optimal ensemble in our SAXS data and their sequence similarities suggested that NP95 II TTD-PHD exhibits structural similarities with UHRF1 TTD-PHD. Thus, I reasoned that the TTD-PHD linker of NP95 II is able to interact with the TTD domain comparable to UHRF1. 
A
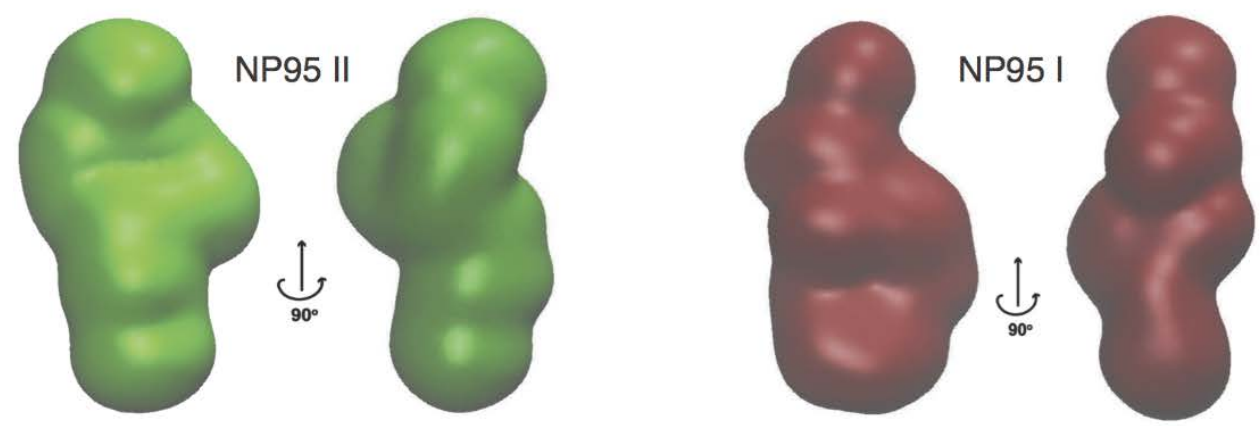

B
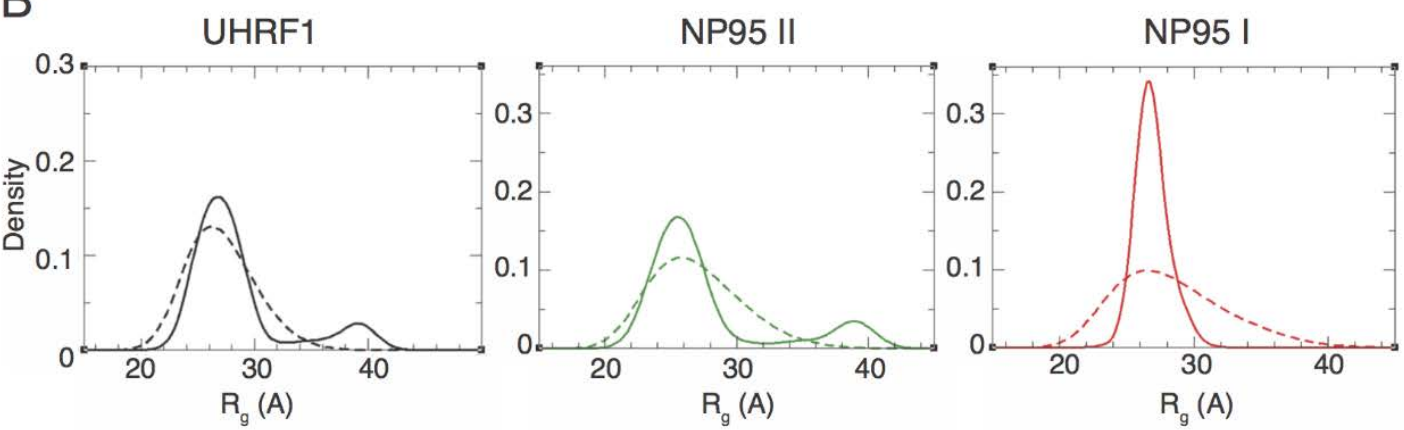

C

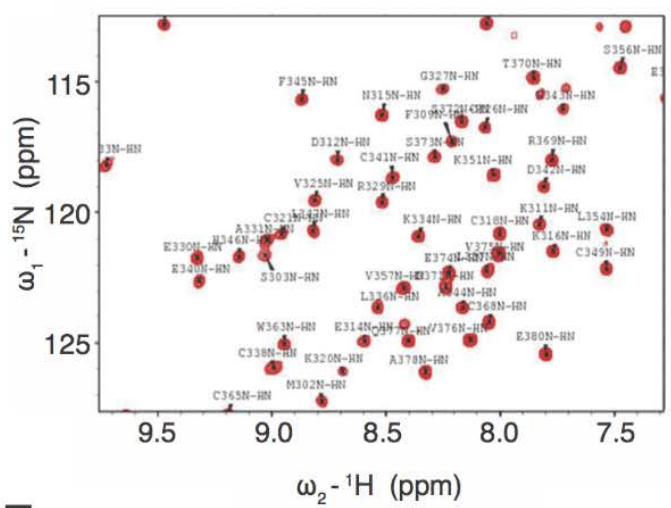

D

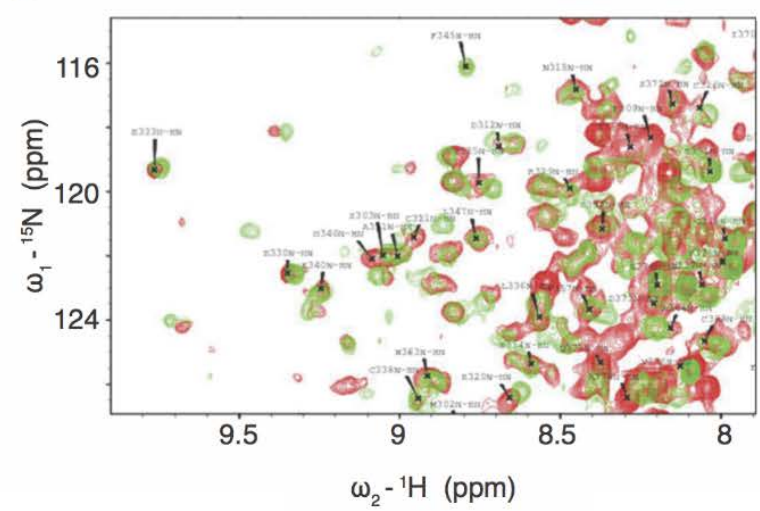

E

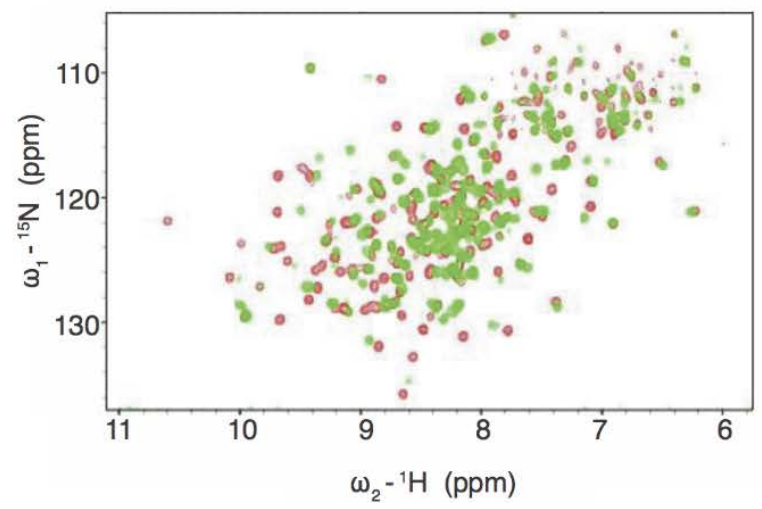

Figure 3-16 Structural differences between TTD-PHD fragments of NP95 I and NP95 II

(A) Abinitio envelope prediction from SAXS curves of wild type TTD-PHD fragments of NP95 I and NP95 II were calculated with DAMMIF. Front view and view $90^{\circ}$ rotated of both fragments are shown. (B) $R_{g}$ distributions of TTD-PHD fragments of UHRF1, NP95 I and NP95 II, theoretical distribution (dashed line) and optimal ensemble distribution (solid line). (C) Assigned HSQC spectra showing residue signals of ${ }^{15} \mathrm{~N}-\mathrm{PHD}$ of 
NP95. (D) TROSY overlay of HSQC spectra showing residue signals of ${ }^{15} \mathrm{~N}-\mathrm{TTD}-\mathrm{PHD}$ fragment of NP95 I and NP95 II highlighted are the assigned PHD resonances. (E) TROSY overlay of HSQC spectra showing residue signals of ${ }^{15} \mathrm{~N}$-TTD-linker fragment of NP95 I and NP95 II. Aleksander Lemak performed SAXS experiments and data analysis. Scott Houliston performed NMR experiments and data analysis (Laboratory of Cheryl Arrowsmith, University of Toronto).

The SAXS data revealed that the rigidity of the TTD-PHD structure of NP95 I was substantially different from NP95 II and UHRF1. The theoretical modeling of the optimal ensemble of the TTD-PHD of UHRF1 and NP95 II showed two distinct peaks, suggesting certain flexibility of the TTD-PHD linker in this context. I reasoned that UHRF1 and NP95 II TTD-PHD share similar structural features. NP95 II TTD-PHD might adapt a multivalent binding mode to $\mathrm{H} 3 \mathrm{~K} 9 \mathrm{me} 3$ with unmodified N-terminus as described for UHRF1 TTD-PHD. In contrast, NP95 I TTD-PHD optimal ensemble distribution only exhibited one sharp peak $\left(\mathrm{R}_{\mathrm{g}}=27\right)$. This finding argued for high rigidity of the TTD-PHD structure and only minimal flexibility of the TTD-PHD linker in this protein.

\subsubsection{TTD-PHD linker causes structural differences in the TTD of NP95 I and NP95 II}

Next, we wanted to study the structural features of the interaction between the TTD surface and the variable adjacent linker regions of NP95. To determine the nature of this interaction and the essential contributing residues in the TTD domain, we used NMR spectroscopy. We were able to assign all peaks of the HSQC spectra of the NP95 PHD domain (Figure 3-16C). Overlay of the plotted resonance peaks revealed the PHD resonances of both isoforms were superimposable (Figure 3-16D). Given these results, we analyzed a region of the NP95 variants only comprising the TTD and the TTD-PHD linker. The result showed great differences between the HSQC spectra of the NP95 I and NP95 II TTD-linker region (Figure 3-16D). However, the additional nine amino acids of the NP95 I linker insertion were not sufficient to explain these great differences of the TTD resonances of the two isoforms. Therefore, we reasoned that the observed differences in the HSQC spectra are due to the TTD-PHD linkers of NP95 I and NP95 II and especially their differential interaction with the TTD domain.

Taken together the result of our structural studies, we found that the TTD-PHD linker of NP95 I exhibited exceptional features in all our assays. The findings imply a high rigidity of the structure of the TTD-PHD region and a direct interaction of the TTD-PHD linker with the TTD domain surface. This interaction is likely to cause the observed blocking of the TTD target binding. 


\subsubsection{Different intramolecular interactions in UHRF1 and the NP95 isoforms}

My results demonstrated that the PBR region had only minor influence on the H3 tail binding of the two NP95 isoforms. However, I showed functional similarities of the NP95 I TTD-PHD linker and the UHRF1 PBR. In addition to that, the TTD-PHD of NP95 II and UHRF1 showed high similarity. Based on these differences between UHRF1, NP95 I and NP95 II, I reasoned that the intramolecular interactions of the different domains within the three proteins must significantly differ from each other. In collaboration with the laboratory of Henning Urlaub (MPI Goettingen, Germany) we performed BS3 protein crosslinking experiments with subsequent liquid chromatography-tandem mass spectrometry analysis to assess the intramolecular interactions in NP95 I, NP95 II and UHRF1. BS3 is crosslinking primary amines; therefore the analysis identified crosslinks connecting lysine residues in different regions of the protein.

\subsubsection{Common intramolecular crosslinks of NP95 and UHRF1}

Comparison of multiple data sets for all three proteins revealed similarities in the intramolecular connections of UHRF1 and the two NP95 isoforms. We found that in all three proteins the SRA domain is establishing many matching contacts inter alia with the UBL domain, the UBL-TTD linker, the PHD-SRA linker, and the PBR (Figure 3-17A, B, C, dark blue lines). In addition to that, we reproducibly found crosslink connections between the TTD domain and the SRA domain of all three proteins (Figure 3-17A, B, C, bold dark blue lines).

In our previous experiments we had found a role for the PBR region in regulation of the TTD/H3K9me3 interaction in UHRF1. The crosslinking experiments validated these results. We found crosslinks connecting the TTD domain with the PBR in UHRF1 (Figure 3-17A, bold dark blue lines). Importantly, we detected similar crosslink contacts between the PBR and the TTD in NP95 I and NP95 II (Figure 3-17 B, C, bold dark blue lines). However, we found no overlap of exact crosslink positions in the TTD and the PBR comparing UHRF1 and NP95.

\subsubsection{Different crosslink positions in the NP95 isoforms}

The results showed great differences in the H3K9me3 binding of NP95 I and NP95 II (Figure 3-8; 3-11). Structural analysis of the TTD-PHD regions of the two proteins additionally revealed different interactions of the TTD-PHD linker region with the surface of the TTD domain. We wanted to analyze if these differences influence the 
intramolecular interactions in the proteins. Thus, we compared the intramolecular crosslinks of NP95 I and NP95 II.

A

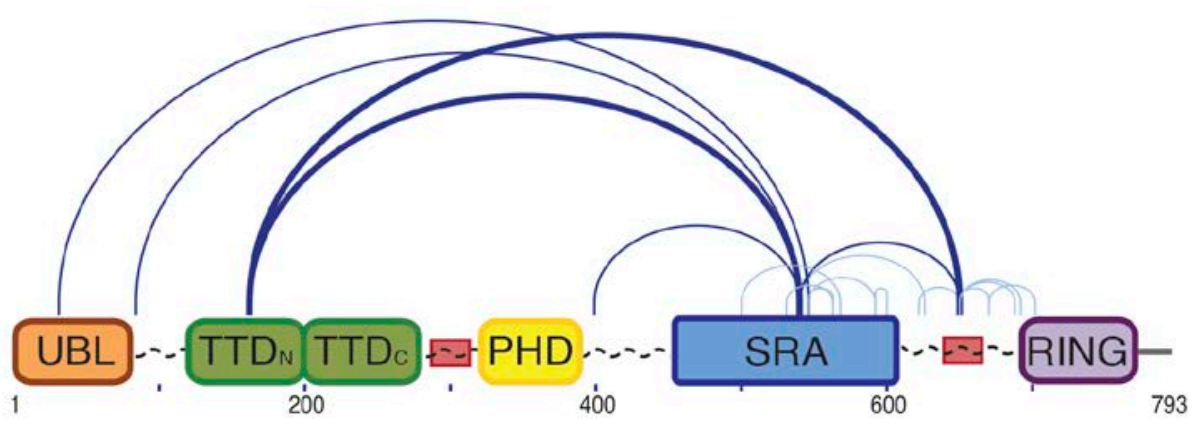

B

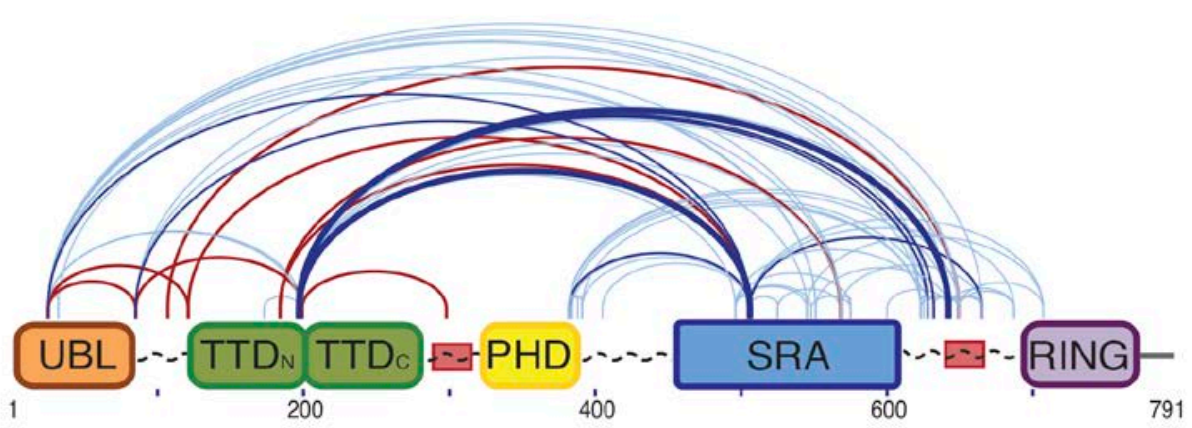

C

NP95 I

NP95 ॥

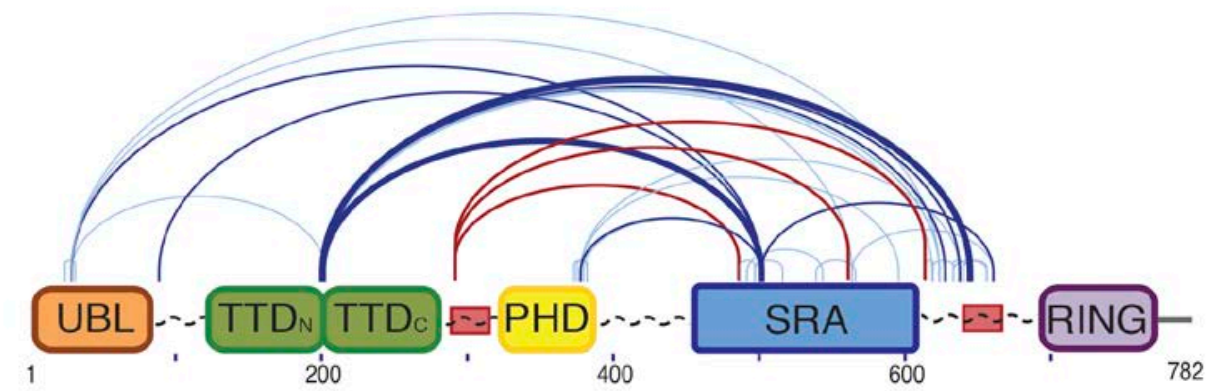

Figure 3-17 Intramolecular crosslinking of UHRF1, NP95 I and NP95 II

Schematic representation of the domain organization of UHRF1 (A), NP95 I (B) and NP95 II (C); recombinant proteins were crosslinked with BS3, digested and analyzed by LC MS/MS. Arcs represent crosslink positions identified in the monomeric proteins. Common positions of UHRF1, NP95 I and II (dark blue line) are highlighted especially when TTD residues are involved (bold dark blue line). Unique crosslink positions are represented (red line). All shown crosslinks were identified with a score greater than 4.0. Experiment and data analysis was performed by Aleksander Chernev (Laboratory of Henning Urlaub, MPI-bpc Goettingen)

We found substantial differences in the positioning of the crosslink connections in NP95 I and II (Figure 3-17B, C). We found that in NP95 I unique contacts were established involving the TTD itself or the close proximity of the domain (Figure 3-17B, red lines). In 
agreement with my findings the TTD-PHD linker of NP95 I impaired the H3K9me3 binding of the TTD domain, we detected one crosslink indicating a connection between the TTD and the TTD-PHD linker of NP95 I. In addition to that the crosslinking pattern showed close interactions of the TTD with the N-terminus of the protein as well as connections of the UBL-TTD linker with the SRA and the PBR. We reasoned that the unique contacts found in the protein are a result of the insertion in the TTD-PHD linker. Analyzing the NP95 II intramolecular contacts, we reproducibly found a unique crosslink connection between the TTD-PHD linker RKS motif and the SRA domain (Figure 3-17C, red line).

\subsubsection{DNA induced changes in intramolecular crosslinking of UHRF1/ NP95}

It was reported before that the NP95 I binding of hemimethylated DNA to the SRA domain slightly enhanced the binding to H3K9me3 (Pichler et al., 2011). I reasoned that DNA binding to the protein might be able to affect the block of the TTD domain by the TTD-PHD linker. In addition to that, our intramolecular crosslinking results showed connections between the SRA domain and the TTD domain indicating close interactions of both domains in UHRF1 as well as NP95 (Figure 3-17). I reasoned that such interactions could point to an interrelation of the binding processes of both domains in all three proteins.

Thus, we wanted to know if the intramolecular interactions of the three proteins are also affected in a different manner to analyze the changes of intramolecular interactions upon DNA binding. We performed intramolecular crosslinking experiments adding hemimethylated DNA (same as used in the above experiments) and compared the results with our former experiments.

Compared to the previous experiments, we found a substantial loss in total number of specific crosslink positions for UHRF1 (24\%), NP95 I (48\%) and NP95 II (60\%) upon addition of hemimethylated DNA. Comparing the resulting crosslink contacts of UHRF1 with DNA to the sample without, we determined a loss of 15 crosslink positions (see data tables in supplement). Ten out of these 15 involved SRA residues, while nine contacts were established by the PBR. The results showed that all previously described connections between TTD and PBR were lost upon addition of hemi-methylated DNA. At the same time, additional crosslinks were established between K650 (PBR) and K294 (TTD-PHD linker) as well as K303 (pre-PHD) upon DNA titration. Thus, lost connections of the PBR region with the TTD might be shifted to the TTD-PHD linker as well as the pre-PHD. 
In NP95 I, comparison of our findings with and without DNA revealed a number of 33 crosslinks lost upon addition of hemimethylated DNA. As for UHRF1, most of the lost connections involved SRA residues. Additionally, we recognized a loss of crosslink positions that were established by TTD and SRA-RING linker. In contrast to UHRF1, we found no complete loss of crosslink connections between TTD and PBR of NP95 I.

In NP95 II, presence of hemimethylated DNA caused a loss of crosslink connections that involved SRA domain lysine residues. Out of 35 crosslink contacts that were lost upon DNA addition, 30 involved SRA residues. Therefore, NP95 II lost 94\% of its intramolecular crosslinks with the SRA domain. The crosslink connections that were found between the TTD-PHD linker and the SRA in our former experiments (Figure 3-17C) were not found after addition of DNA, while the contacts between TTD and SRA-RING linker seemed rather unaffected.

In general we found that the addition of DNA mainly affected the connections involving SRA residues. In UHRF1, we also found great changes of PBR crosslink connections with the TTD of the protein. These crosslinks were not found upon addition of hemimethylated DNA. Thus, DNA binding affected the TTD/PBR interaction. In contrast, we found no such effect in NP95 I or NP95 II. Upon DNA addition the murine proteins mainly lost crosslink connections involving residues of the SRA domain. This argues that all three proteins were differently affected by the addition of hemimethylated DNA.

\subsubsection{Impact of DNA on the $\mathrm{H} 3$ tail binding of UHRF1/NP95}

My former results showed that UHRF1, NP95 I and NP95 II intramolecular crosslinking connections were influenced differently by the addition of hemimethylated DNA. The intramolecular crosslinking results showed connections between the SRA domain and the TTD domain were lost in UHRF1 upon addition of DNA (Chapter 3.2.6.3). I reasoned that such loss interactions could point to an interrelation of the binding processes of both domains. Therefore, I wanted to determine the effects of the addition of hemimethylated DNA on H3 tail binding in UHRF1 and the two NP95 isoforms (Figure 3-18A).

I used fluorescence polarization binding experiments to determine $\mathrm{H} 3$ tail peptide binding affinity of UHRF1 and NP95 after pre-incubation with hemi-methylated 12-mer DNA. I compared the peptide binding behavior of protein incubated with DNA with protein that was incubated with the DNA hybridization buffer. I analyzed the impact on the TTD and the PHD by using the discussed peptides exclusively targeting each of the domains. 

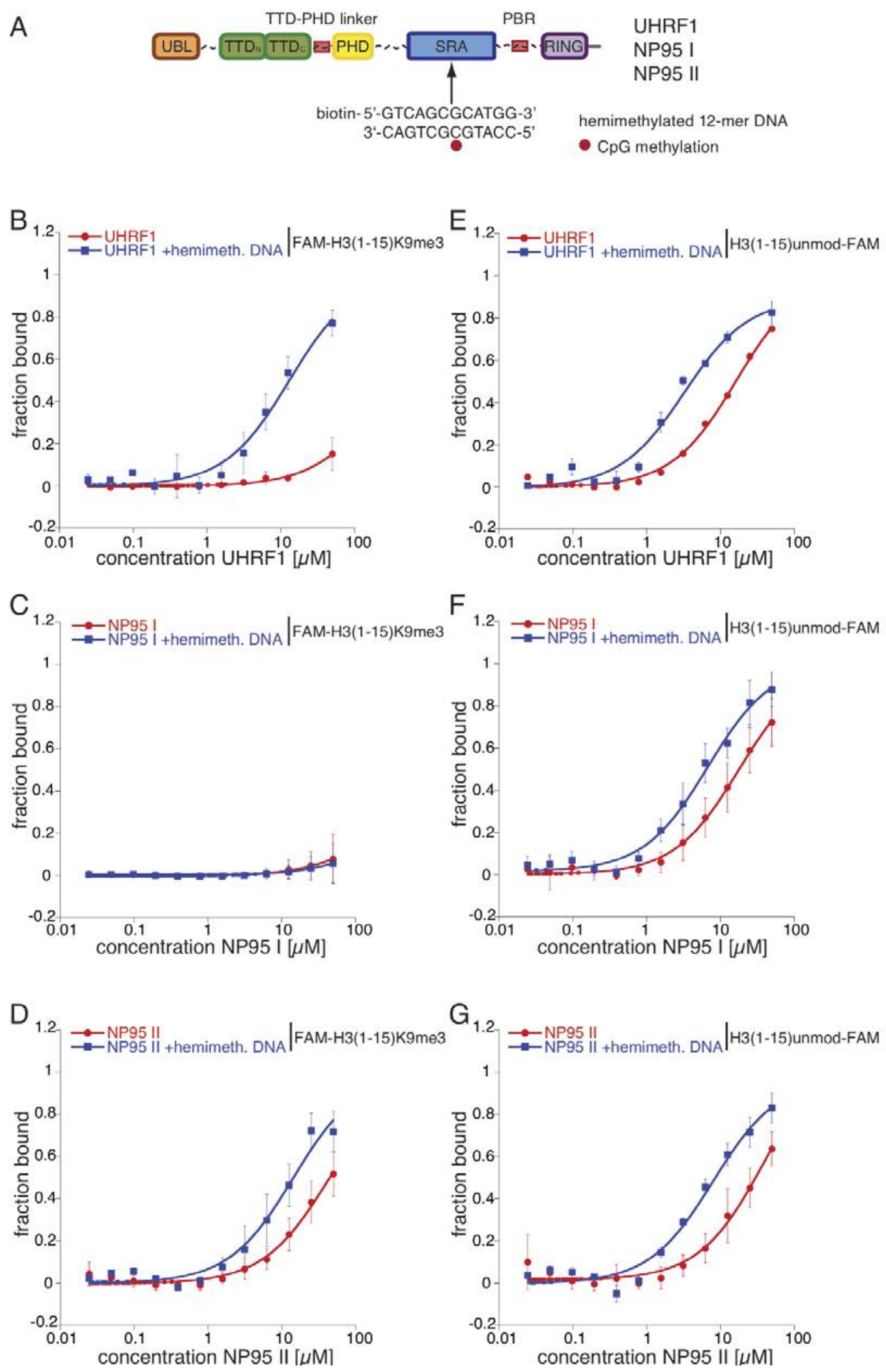

Figure 3-18 Impact of DNA on the H3 tail binding of UHRF1/ NP95

(A) Schematic representation of UHRF1/NP95 domain architecture. The hemimethylated 12mer oligonucleotide used for the experiments is shown. 5-Methylcytosine site is marked by a red dot. (B, E) UHRF1, (C, F) NP95 I and (D, G) NP95 II were used in fluorescence polarization binding experiments with the indicated FAM-labeled peptides with and without 2-fold molar excess of hemimethylated 12mer DNA. Fraction bound of labeled peptides was plotted depending on protein concentration. Error bars represent standard deviation of at least three independent experiments. 
The presence of DNA to UHRF1 had striking effects on the binding to FAM-H3(1-15)K9me3 $(\mathrm{Kd}=12.9 \mu \mathrm{M})$ (Figure 3-18B, Table 3-3). The interaction of TTD with the peptide improved more than 10-fold upon addition of 2-fold molar excess of hemi-methylated DNA. In contrast, the hemimethylated DNA had no effect on the NP95 I TTD binding to K9 trimethylation (Figure 3-18C) and only moderate effects on the NP95 II TTD $(\mathrm{Kd}=14.0 \mu \mathrm{M})$ (Figure 3-18D). At the same time, there was a slight gain of binding of the PHD to the H3(1-15)unmod-FAM in all three proteins (Figure 3-18E, F, G).

Taken together the results demonstrated that the addition of DNA had different effects on the binding of the TTD of UHRF1 or the NP95 variants to K9 trimethylation but similar effects on the PHD interaction with the unmodified H3 N-terminus.

Table 3-4 binding affinity of UHRF1 and NP95 to indicated peptides with and without DNA (apparent $\mathrm{Kd}$ values in $\mu \mathrm{M}$ )

\begin{tabular}{lcccc}
\hline & & FAM-H3(1-15)K9me3 & H3(1-15)unmod-FAM & H3(1-15)K9me3-FAM \\
\hline UHRF1 & - DNA & ND & $16.1 \pm 2.3$ & $10.7 \pm 1.9$ \\
& + DNA & $12.9 \pm 3.2$ & $3.2 \pm 0.8$ & $1.9 \pm 0.4$ \\
\multirow{2}{*}{ NP95 I } & - DNA & ND & & \\
& + DNA & ND & $17.4 \pm 2.1$ & $9.9 \pm 0.6$ \\
\multirow{2}{*}{ NP95 II } & - DNA & $39.8 \pm 11.2$ & $6.7 \pm 1.1$ & $8.4 \pm 1.4$ \\
& + DNA & $14.0 \pm 3.9$ & $37.0 \pm 14.9$ & $24.8 \pm 2.7$ \\
\hline
\end{tabular}

\subsection{Expression and cellular localization of NP95}

\subsubsection{Comparison of the nuclear localization of UHRF1 and NP95}

I showed substantial differences in the behavior of UHRF1, NP95 I and NP95 II in regard to histone binding alone and the interplay of histone and DNA binding. Thus, I wanted to investigate if these differences were reflected by their cellular localization. UHRF1 localization was shown to be dependent on the ability of the TTD to interact with trimethylated H3 tails and the SRA to interact with DNA (Figure 3-2C). In addition to that, we found the PBR to impair the recruitment of the protein to $\mathrm{H} 3 \mathrm{~K} 9 \mathrm{me} 3$ rich loci (Figure 3-4D, E). When analyzing the UHRF1 nuclear localization in human cells, we found that only a small fraction of cells showed co-localization of the protein with H3K9me3 (Figure 3-1). 
mCherry
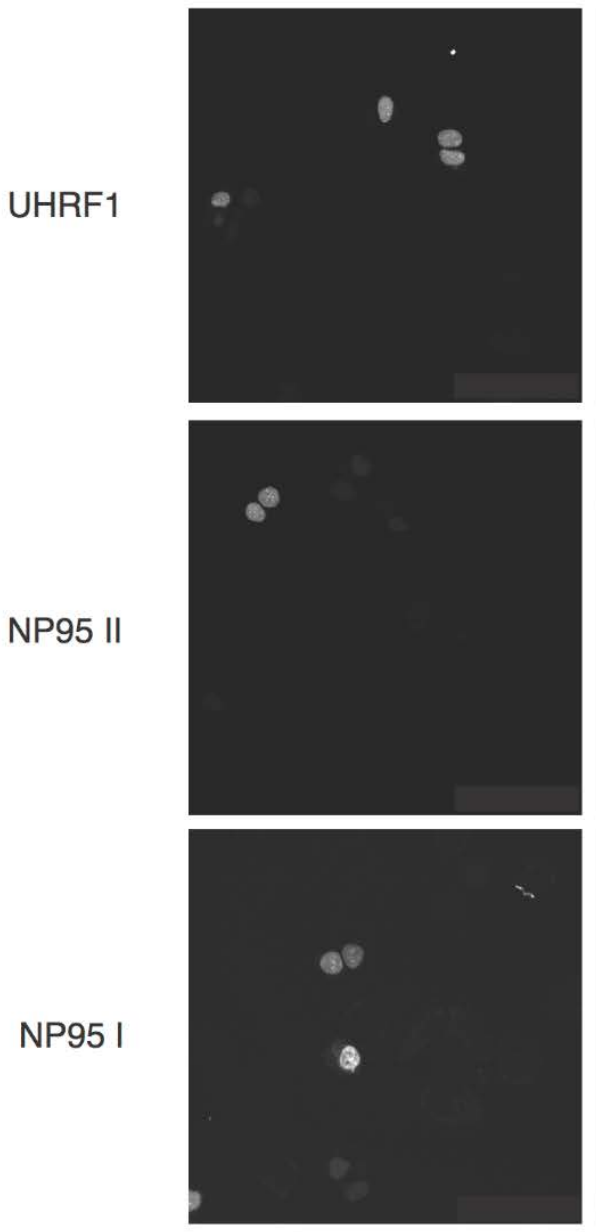

8

Lac I
H3K9me3
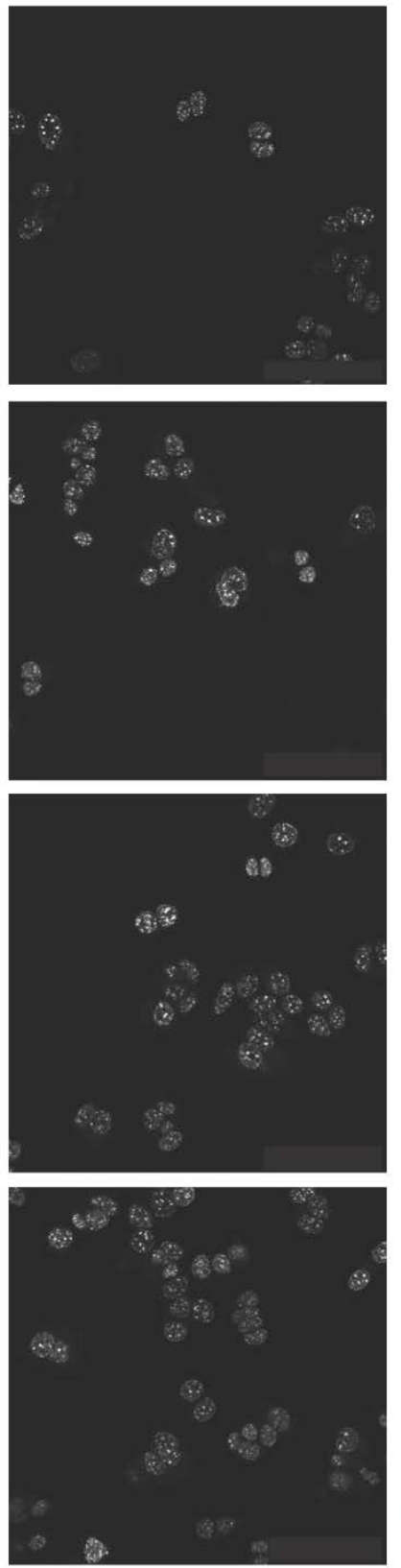

merge

mCherry, H3K9me3
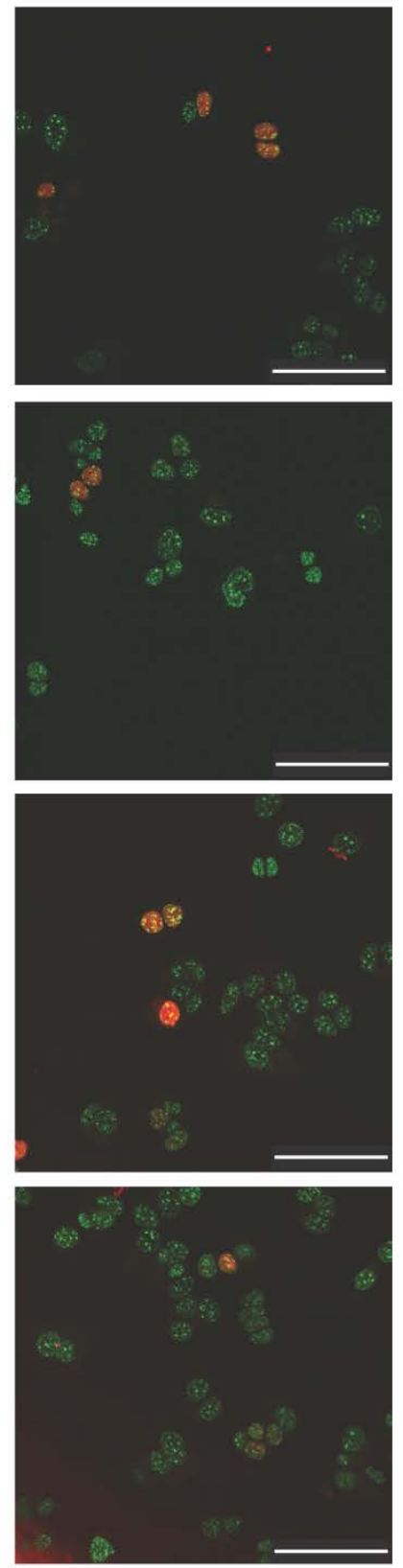

Figure 3-19 Co-localization of UHRF1, NP95 I and NP95 II with H3K9me3 in MCF7 cells

Co-localization of UHRF1, NP95 I and II and H3K9me3 modification were analyzed in MCF7 cells. MCF7 cells cells transiently expressed mCherry-tagged UHRF1, NP95 II, NP95 I or Lac I. H3K9me3 was detected using specific antibodies. Microscopy images of separate and merged fluorescence channels (UHRF1-red, H3K9me3green) are shown. Scale bar represents $75 \mu \mathrm{m}$. Experiments were done in collaboration with Sarah Kreuz (Laboratory of Wolfgang Fischle, MPI, Goettingen).

In my studies, I compared the localization of mCherry-tagged UHRF1 and NP95 isoforms in cells. I found considerable differences in their subnuclear localization in human cells. In collaboration with Sarah Kreuz from the Laboratory of Wolfgang Fischle (MPI, Goettingen), I quantified these differences. We determined the fraction of cells that 

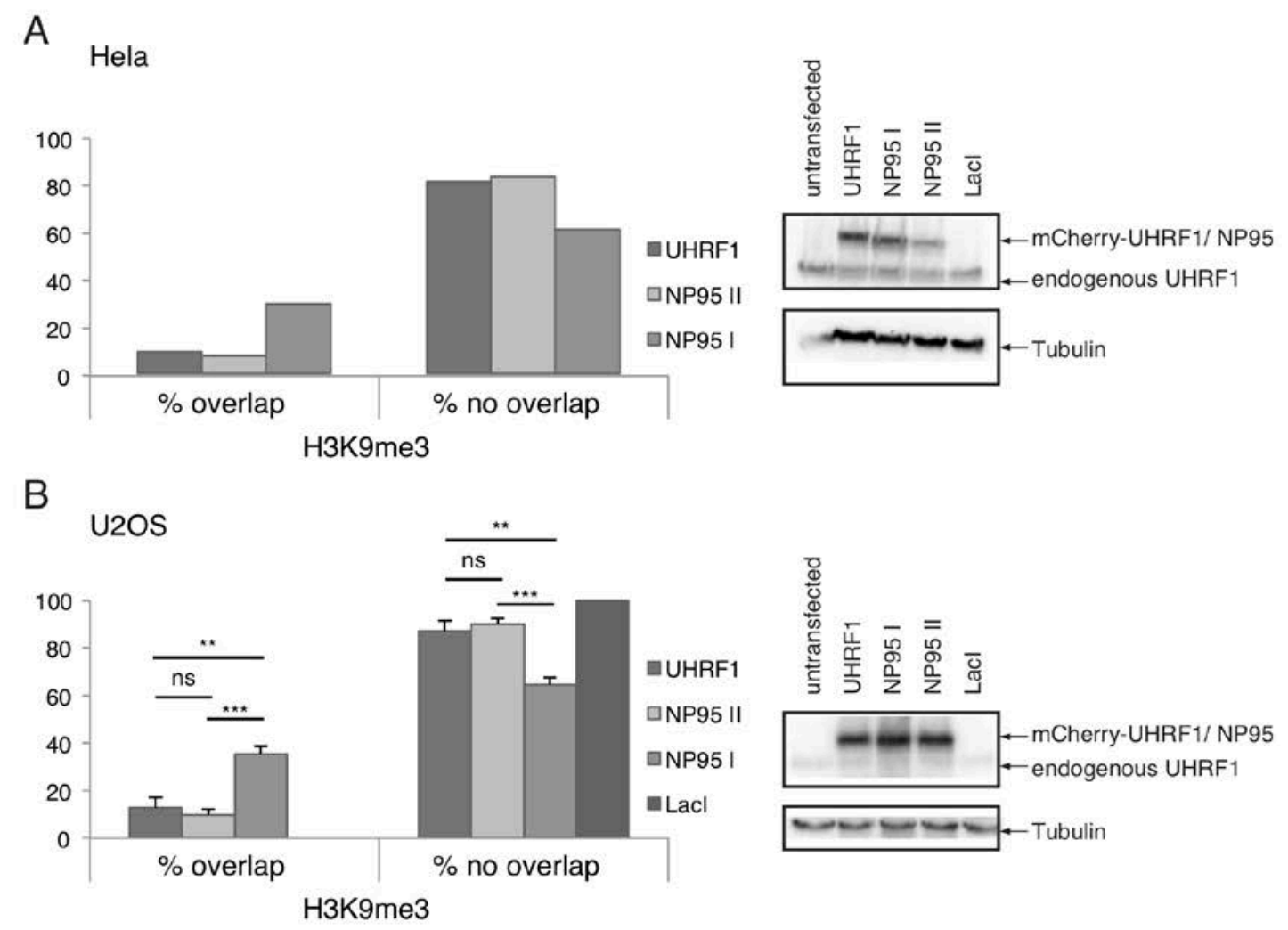

\section{C}
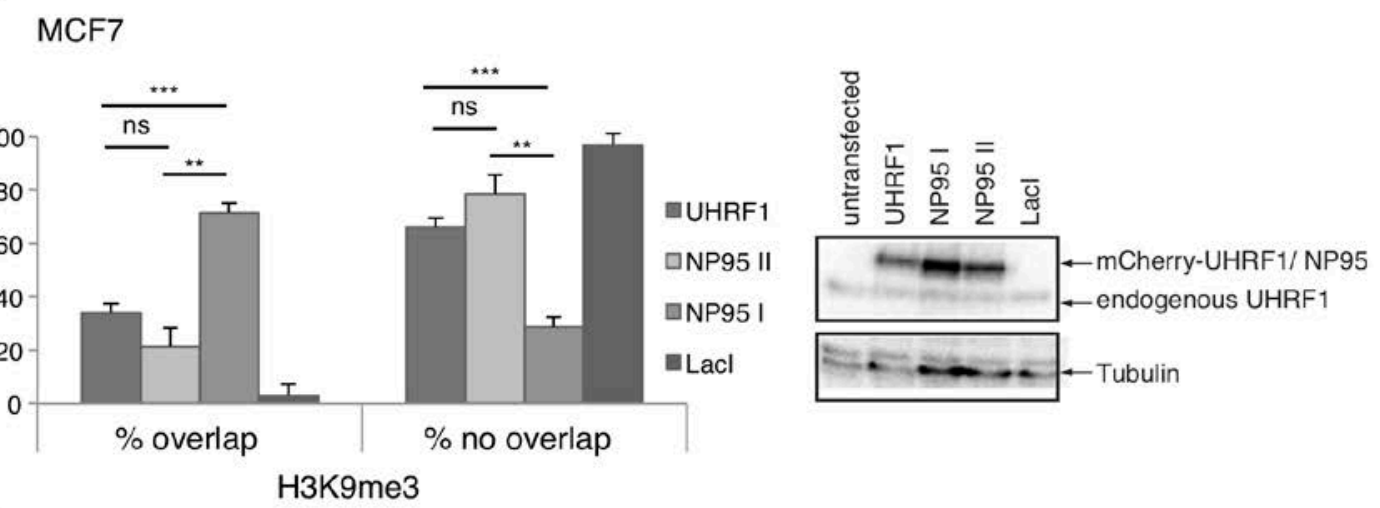

D

\section{NIH3T3}
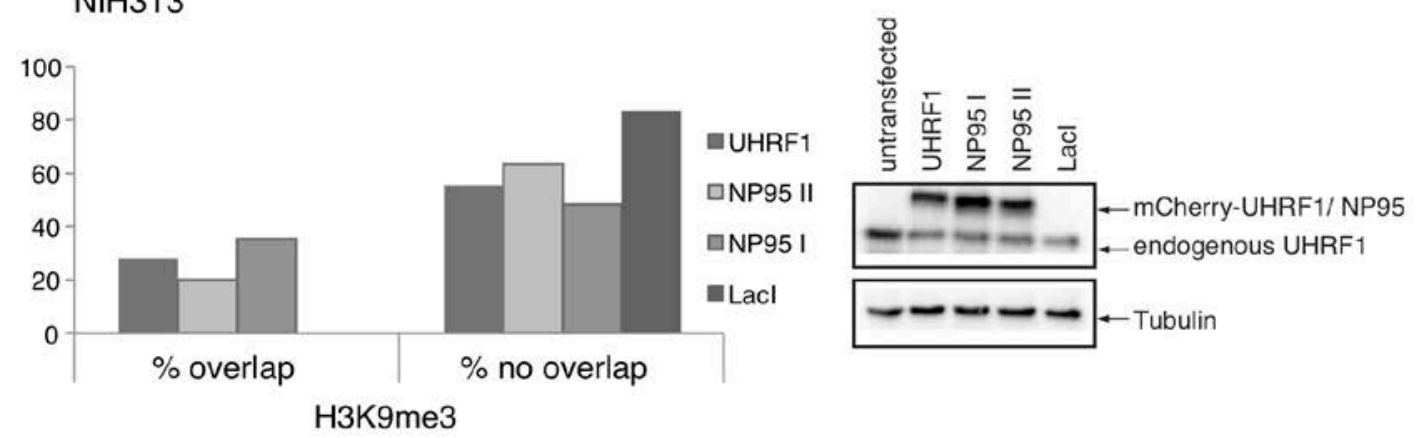

Figure 3-20 Comparison of the nuclear localization of UHRF1 and NP95

(A) Hela, (B) U2OS, (C) MCF7 and NIH3T3 (D) cells were transfected with mCherry-tagged UHRF1, NP95 I or II after transfection nuclear cell extracts were analyzed western blot (right panel). Cells were than analyzed by fluorescence microscopy and H3K9me3 was visualized using indirect immunofluorescence. Overlap of mCherry-tagged UHRF1 or NP95 was determined and plotted (left site histogram). All error bars show standard deviation of three independent experiments. Asterisks show significance and ns, not significant. Data was generated in collaboration with Sarah Kreuz (Laboratory of Wolfgang Fischle, MPI, Goettingen) 
exhibited co-localization of mCherry-UHRF1, mCherry-NP95 I or mCherry-NP95 II with the H3K9me3 modification using fluorescence microscopy and subsequent cell counting (Figure 3-19). Regardless of the differences in the overall expression levels of the three proteins, we found a reproducible pattern (Figure 3-20A, B, C). In HeLa cells only a small fraction of cells showed co-localization of mCherry-UHRF1 with H3K9me3 (Figure 3-20A). We observed similar results for cells that were transiently transfected with mCherry-NP95 II. In contrast, a greater fraction of cells exhibited an overlap of mCherryNP95 I with the modification signal. We found the same pattern in U2OS (Figure 3-20B) and MCF7 cells (Figure 3-20C). Similar experiments in the murine cell line NIH3T3 led to the same result (Figure 3-20D).

In cells, NP95 I is co-localized with H3K9me3, while binding to the modification was blocked in vitro. NP95 II TTD binding to H3K9me3 was not blocked in our in vitro experiments. Nevertheless, we found less co-localization of NP95 II with the modification in cells. At the same time, UHRF1 showed a stronger co-localization with H3K9me3 than NP95 II and less co-localization with the modification than NP95 I. Based on these results, I concluded that the different inter-domain linker regions influence the subnuclear localization of the proteins.

\subsection{2 mRNA expression analysis of NP95 I and NP95 II}

Since NP95 I and NP95 II exhibited different co-localization with H3K9me3, I reasoned that both proteins might have distinct nuclear target sites and thus might fulfill different functions. Therefore, we investigated the expression of the two murine protein variants in different tissues. In collaboration with Klaus Jung (UMG, Goettingen, Germany) we performed a retrospective analysis of RNA-Seq data. The data used in this analysis were publicly available datasets obtained from encode (http://genome.crg.es/encode_RNA _dashboard/mm9). The "Uhrf1-001" and "Uhrf1-004" were mRNA sequences encoding for full-length NP95 I and the "Uhrf1-002" and "Uhrf1-003" were mRNAs encoding fulllength NP95 II. “Uhrf1-005”, “Uhrf1-006” and “Uhrf1-007” were RNA sequences encoding for shorter isoforms of NP95, which were barely expressed in all samples.

The first dataset consisted of 99 samples of Poly-mRNA, paired-end reads with quality measure. The dataset included replicates of 17 different types of tissues. The data showed that NP95 I ("Uhrf1-001" and "Uhrf1-004") exhibited the highest expression in adult thymus (ThymusAdult8wks) as well as in testis (TestisAdult8wks), ovary (OvaryAdult8wks), and the large intestine (LgintAdult8wks) (Figure 3-21). Additionally we found low to mediocre expression of NP95 I and NP95 II in adult duodenum 


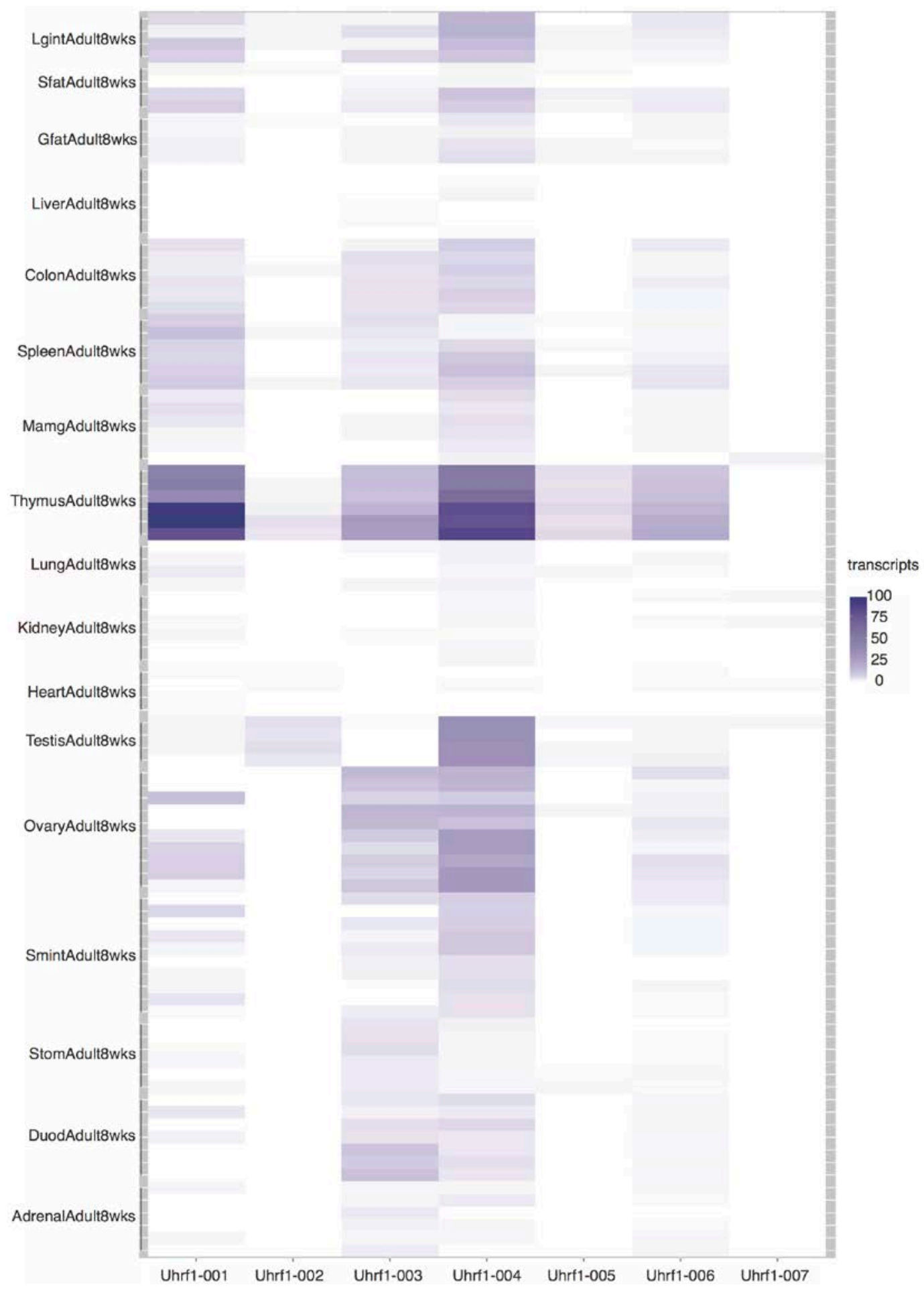

Figure 3-21 Retrospective mRNA expression analysis of 17 different types of murine tissues

Expression heat map of the first dataset for 99 samples against the 7 transcript isoforms, dataset was obtained from encode (http:/ / genome.crg.es/encode_RNA_dashboard/mm9), number of NP95 transcripts relative to 1 million total mRNA transcripts was analyzed and plotted, "Uhrf1-001" and "Uhrf1-004" were mRNA sequences encoding for full-length NP95 I, the "Uhrf1-002" and "Uhrf1-003" were mRNAs encoding fulllength NP95 II, "Uhrf1-005", "Uhrf1-006" and "Uhrf1-007" were mRNAs encoding for not full-length isoforms, sample identification by tissue and age is indicated (left site), transcript isoforms are shown (bottom),samples were analysed from long intestine (Lgint), subcutaneous fat (Sfat), liver, colon, spleen, mammary gland, thymus, lung, kidney, heart, testis, ovary, small intestine (Smint), stomach (Stom), duodenum (Duod) and adrenal gland (Adrenal), all samples came from a 8 week old adult animal (Adult8wks). The data analysis was performed by Alexander Wolff (UMG, Goettingen) 


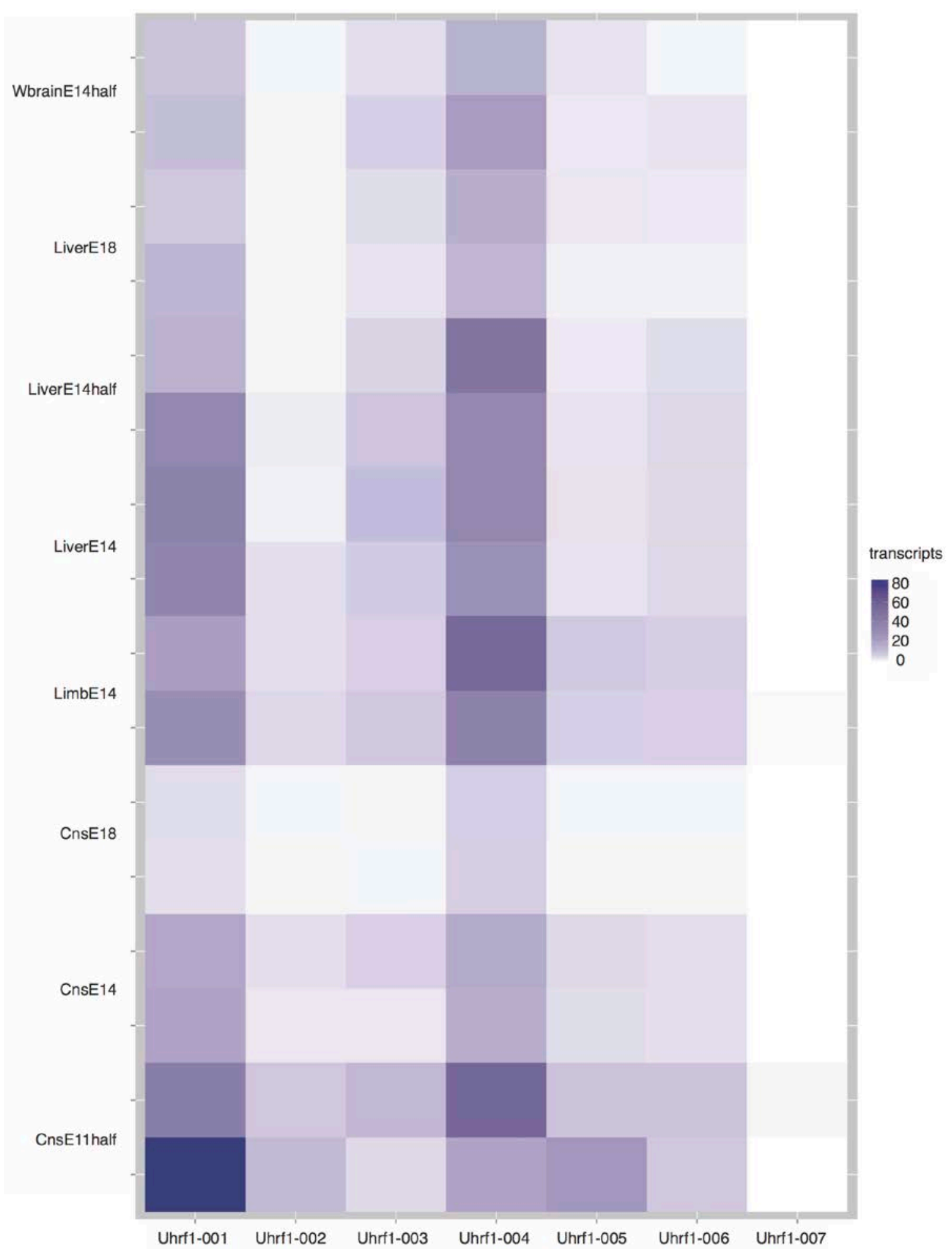

Figure 3-22 Retrospective mRNA expression analysis of different embryonic developmental stages

Expression heat map of the second dataset for 16 samples against the 7 transcript isoforms, dataset was obtained from encode (http://genome.crg.es/encode_RNA_dashboard/mm9), number of NP95 transcripts relative to total mRNA was analyzed and plotted, "Uhrf1-001" and "Uhrf1-004" were mRNA sequences encoding for full-length NP95 I, the "Uhrf1-002" and "Uhrf1-003" were mRNAs encoding full-length NP95 II, "Uhrf1-005", "Uhrf1-006" and "Uhrf1-007" were mRNAs encoding for not full-length isoforms, transcript isoforms are shown (bottom), sample identification by tissue and age is indicated (left site), samples came from whole brain (Wbrain), liver, limb and central nervous system, from 11.5, 14, 14.5 and 18 days old embryos (E11half, E14, E14half, E18). The data analysis was performed by Alexander Wolff (UMG, Goettingen) 
(DuodAdult8wks), subcutaneous fat tissue (SfatAdult8wks), small intestine (SmintAdult8wks) and spleen tissue (SpleenAdult8wks). In contrast to that we detected no expression of any transcript isoforms in adult adrenal gland tissue (AdrenalAdult8wks), heart (HeartAdult8wks), kidney (KidneyAdults8wks), liver (LiverAdults8wks), and lung (LungAdult8wks). The second dataset was a deep-sequence dataset of eight different embryonic stages and tissues with two replicates for a total of 16 samples. Analysis of the second dataset revealed that NP95 I "Uhrf-001" and "Uhrf1$004 ")$ were the primarily expressed transcripts in samples of embryonic day 11 central nervous system (CnsE11half), embryonic day $14 \mathrm{limb}$ (LimbE14) and liver (LiverE14) as well as embryonic day 14.5 liver (LiverE14half) (Figure 3-22). The NP95 II transcripts were either low or not expressed in the different embryonic stages and tissue-types.

UHRF1 mRNA is reported to be most abundant in human thymus and fetal liver but also highly expressed in bone marrow, lung, kidney, small intestine and testis (Hopfner et al., 2000). Our results demonstrated comparable expression for NP95 I. It was most abundant in murine thymus and embryonic liver and was also highly expressed in ovary, testis, small intestine as well as large intestine. This might argue for similar cellular functions of UHRF1 in human and NP95 I in mouse. In contrast, NP95 II showed only low expression in all tissues analyzed. Given my finding that NP95 I and II show different co-localization with H3K9me3 in cells, I concluded that NP95 I and NP95 II might have distinct functions in the nucleus and, therefore, have variable expression patterns in different cell types. 


\section{Discussion}

The combinatorial interaction of different domains with a complex target containing multiple ligands is referred to as multivalent binding. The first concept of multivalent readout of epigenetic marks ('modification cassettes') was introduced in 2003 and revealed a putative connection between different modifications that are in close proximity to each other (Fischle et al., 2003). Recent studies showed that multidomain proteins comprising several chromatin interacting domains are capable of combinatorial readout of epigenetic modifications of histones and DNA. Thus, this facilitates crosstalk of these marks (Du and Patel, 2014). This crosstalk is a fundamental element of the 'histone code' theory that postulates that patterns of chromatin marks constitute a complex signalling system (Strahl and Allis, 2000).

UHRF1 is a multidomain protein that has three different domains to interact with different epigenetic modifications and it has been shown to localize to differently modified loci in cells. Recent findings imply that the intramolecular interactions between different inter-domain linker regions and the TTD domain of UHRF1 play a critical role in regulating the binding specificity of the protein for specific marks in vitro (Arita et al., 2012; Cheng et al., 2013; Gelato et al., 2014; Rothbart et al., 2013; Xie et al., 2012). This uncovered that multivalent read out by multidomain proteins is not sufficiently described by a 'beads-on a-string' model based on simple co-localization of chromatin binding domains. Instead, our results exemplified that inter-domain linker regions provide a level of regulation for the combinatorial readout of epigenetic marks. Based on these findings, we proposed a new model for dynamic regulation of multidomain protein interaction with combinatorial chromatin modifications.

\subsection{Model for linker-dependent allosteric transition in UHRF1}

Our previous study demonstrated that the interactions of two inter-domain linker regions of UHRF1, PBR and TTD-PHD linker, are mutually exclusive events and establish two distinct conformational states of the protein (Figure 4-1A, B) (Gelato et al., 2014; Tauber and Fischle, 2015). These different conformational states can be characterized by their different binding specificity for H3K9me3.

In one binding mode, The UHRF1 TTD peptide binding groove accommodates the TTD-PHD linker as shown in context of the isolated TTD-PHD fragment. NMR results have shown that the linker R-K-S motif interacts with the TTD residues, stabilizing the relative positioning of the TTD and PHD. Thus, the TTD-PHD linker was shown to 
generate a scaffold and establish a multivalent binding of TTD and PHD (Figure 4-1B) (Arita et al., 2012; Cheng et al., 2013; Rothbart et al., 2013; Xie et al., 2012). This binding mode is characterized by the simultaneous binding of PHD and TTD to the same H3 tail. The TTD-PHD linker peptide displayed a moderate binding affinity ( $\mathrm{Kd}=30 \mu \mathrm{M})$ to the TTD in vitro (Gelato et al., 2014).

In the other binding mode of UHRF1, the PBR interacts with the TTD peptide binding groove. In this state, only the PHD interacts with the unmodified N-terminus of the $\mathrm{H} 3$ tail, while the TTD binding to H3K9me3 is blocked (Figure 4-1A) (Gelato et al., 2014). The isolated TTD domain exhibits comparable binding affinity to PBR and H3K9me3 peptide. Fluorescence polarization binding experiments displayed a strong interaction between the TTD domain and the PBR peptide $(\mathrm{Kd}=3.5 \mu \mathrm{M})$ (Gelato et al., 2014).

We think that depending on, which linker is associated with the TTD the protein adopts different conformational states (Tauber and Fischle, 2015). Due to the weaker affinity to the TTD, the TTD-PHD linker might not be able to compete with the PBR in the ground state of the protein (Gelato et al., 2014). In our previous study we demonstrated that mutation of the PBR and upon binding of PI(5)P the PBR dependent block of the TTD can be released. Therefore, we reasoned that UHRF1 is able to switch between these two conformations in response to specific external stimuli. This process leads to a delicate equilibrium of the described states. Regulatory processes targeting the mutually unstructured linker regions alter this equilibrium (Gelato et al., 2014).

\subsection{Inter-domain linker dependent regulation of NP95 binding to H3K9me3}

\subsubsection{The PBR dependent blocking of the TTD in UHRF1 is not conserved in the two NP95 isoforms}

In this study, I show that, in contrast to UHRF1, the PBR region does not regulate the H3K9me3 binding of NP95. Mutagenesis of the PBR consensus sequence in NP95 has only minor effects on the $\mathrm{H} 3$ tail binding of the protein. In addition to that, PBR-RING did not cause a decreased binding affinity of NP95 TTD for H3K9me3 in fluorescence polarization measurements, as shown for UHRF1. Nevertheless, we reproducibly found connections between lysine residues of the PBR region and the TTD domain in intramolecular crosslinking analysis. This suggests a different role of the PBR in NP95 that remains elusive. Therefore, the PBR dependent regulation of binding specificity that we described for UHRF1 can not be used as a blueprint to explain NP95 binding behavior. However, 96 
others and I showed recently that the PBR region plays an important role in accurate recruitment of UHRF1 to chromatin (Chu et al., 2012; Gelato et al., 2014). Given the high level of functional conservation of UHRF1 and NP95, this argues that similar regulatory mechanisms might exist in murine NP95. Indeed, two different splicing variants of NP95 are expressed in mice that exhibit differential interactions with the $\mathrm{H} 3$ tail.

\subsubsection{The binding states of UHRF1 are resembled by the two splicing variants of NP95 in vitro}

In this study, I demonstrate that the two H3 tail binding modes identified for UHRF1 are substituted by the two isoforms of NP95 in mouse (Figure 4-1). In the first binding state the TTD is not interacting with the H3K9me3 (UHRF1 A and NP95 I) and in the second binding state the TTD and the PHD together establish multivalent binding mode (UHRF1 B and NP95 II).

The linker insertion in NP95 I prevented the TTD from interacting with H3K9me3 in context of the TTD-PHD region as well as the full-length protein (Figure 4-1C). Transfer of the linker insertion into UHRF1 TTD-PHD domain also led to reduced interaction with H3K9me3 peptides. Importantly, our fluorescence polarization binding experiments reveal an essential role of the proline patch (P293-P294-P295) and the center arginine residue (R298) in blocking the binding of the TTD to H3K9me3. Mutagenesis of these residues to alanine results in release of the TTD and to a gain in H3K9me3 binding in context of the TTD-PHD-fragment of NP95 I. Therefore, NP95 I TTD-PHD linker resembles the mode of action of the PBR in UHRF1 (Figure 4-1 A, C).

In context of the full-length NP95 I the same mutations do not change the binding to H3K9me3 to the same extent as seen in the TTD-PHD fragment, suggesting an additional intramolecular interaction stabilizing the TTD/linker interaction. Our intramolecular crosslinking experiments revealed interactions of the TTD domain with the PBR region, the SRA domain and the N-terminus of NP95 I. These interactions might stabilize the TTD-PHD linker in context of the full-length protein and thus retain the block of the TTD domain.

In contrast to NP95 I, the TTD-PHD linker of NP95 II did not impair the TTD target interaction. The TTD-PHD linker of NP95 II has higher sequence similarity with the TTD-PHD linker in UHRF1 including the essential R-K-S motif. Previous studies revealed an essential contribution of this R-K-S motif to the establishment of a multivalent binding mode of TTD and PHD in UHRF1. This multivalent binding was dependent on the interaction of the TTD-PHD linker with the TTD surface and resulted in a higher binding 
affinity of UHRF1 TTD-PHD to a N-terminally unmodified H3K9me3 peptide (Arita et al., 2012; Rothbart et al., 2013). In context of the TTD-PHD region and full-length NP95 II, the mutation of the R-K-S (R293-K294-S295) motif to alanine impaired multivalent binding of TTD and PHD domain. I reasoned that the mutation might uncouple of both domains. According to that, the TTD-PHD linker might be a scaffold for the concerted binding of TTD and PHD. This is supported by the results of SAXS and NMR experiments that demonstrate similar behavior of the TTD-PHD linker in context of the TTD-PHD domain of UHRF1 and NP95 II. Therefore I reasoned that the multivalent binding mode found in UHRF1 also exists in NP95 II (Figure 4-1B, D).

We found comparable binding states of UHRF1 and NP95 concerning the H3 tail. Yet, DNA binding by the SRA domain strongly influenced H3K9me3 binding UHRF1 but not NP95. Thus, the similarities of the different binding states of the protein in mouse and human are limited to the binding of H3K9me3. Since it was shown that nuclear localization of UHRF1, NP95 I and II is dependent on TTD, PHD and SRA domain in all three proteins, I deduced they have to be regulated in a different manner, due to their differential intramolecular interactions of the TTD with inter-domain linker regions (Rajakumara et al., 2011; Rothbart et al., 2013; Rottach et al., 2010).

\subsection{Inter-domain linker regions regulate the multivalent read out of chromatin marks in UHRF1 and NP95}

\subsubsection{TTD/linker interaction is a prerequisite for a cooperative binding event in UHRF1 but not in NP95}

Emerging evidence indicates that the chromatin binding modules of UHRF1 do not act independently of each other but establish interactions with patterns of chromatin modifications (Arita et al., 2012; Bartke et al., 2010). Indeed, I show that the TTD/PBR interaction in UHRF1 is not only a simple mechanism to block the TTD binding to H3K9me3, but it is more importantly generating the basis of a cooperative binding event of TTD and SRA to H3K9me3 and DNA methylation (Figure 4-1 B). Unlike UHRF1, the cooperative binding of DNA and H3K9me3 is not conserved in NP95. This might be connected with the different role of the PBR in NP95 (Figure 4-1 C, D; Chapter 4.2.1).

In NP95, binding measurements did not detect improved binding of the TTD domain to H3K9me3 in the presence of hemimethylated DNA. Nevertheless, it was shown before that NP95 I binding to H3K9me3 peptide is improved upon addition of hemimethylated DNA (Pichler et al., 2011). The peptides used in this study comprised the amino acids 1 to 98 
20 of the H3 tail and were C-terminally labeled with TAMARA. Thus, I reasoned that the increased binding of NP95 I to the H3(1-20)K9me3-TAMARA might be due to the interaction of the PHD domain with the unmodified N-terminus of H3. The TTD binding to H3K9me3 was unaffected.

In UHRF1, I demonstrated that the presence of hemimethylated DNA restores the TTD/H3K9me3 interaction that was blocked by the PBR region in vitro. Therefore, I reasoned that the binding of the SRA domain to DNA leads to a conformational change in the protein that abrogates the TTD / PBR interaction. Given that the interaction of the TTD with PBR involves a polybasic patch, the interaction could be interfered by the negative charge introduced upon DNA binding. Hence, the weakened TTD/PBR interaction might lead to enhanced binding of TTD to H3K9me3. In this way, UHRF1 establishes a binding mode where it interacts with both the hemimethylated oligonucleotide and H3K9me3. This clearly demonstrates that the inter-domain linker regions are not passive and flexible domain linkage, but are important regulators of UHRF1 binding specificity.

Based on this rational, I reasoned that the interaction of UHRF1 with a regulator that improves binding of the TTD to H3K9me3 might affect the TTD/PBR interaction and increase the binding to DNA methylation by the SRA domain.

A

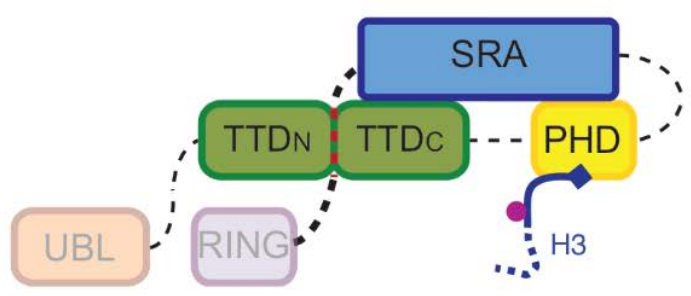

UHRF1 A

B

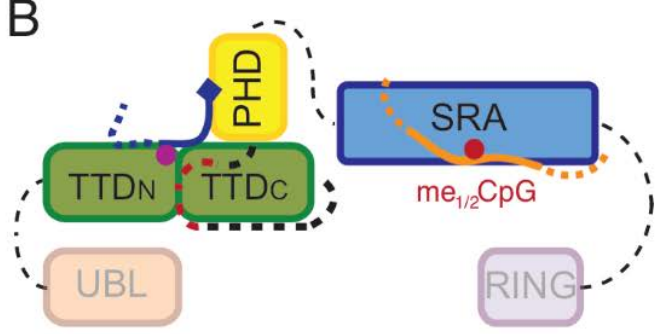

UHRF1 B

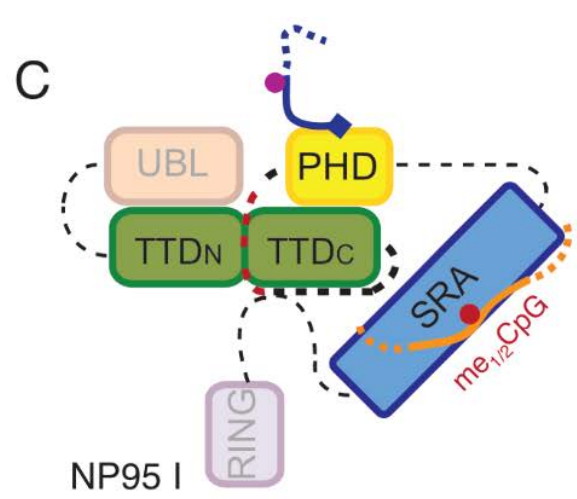

$\mathrm{D}$

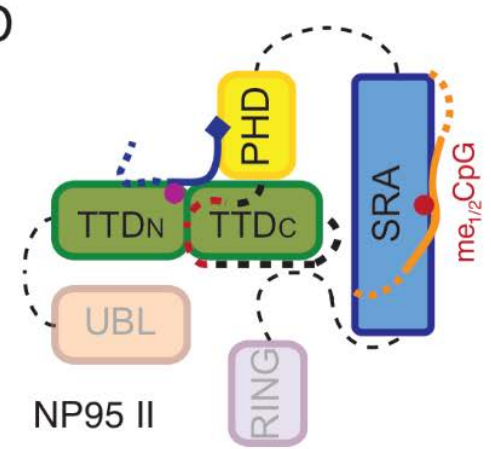

Figure 4-1 Hypothetical models of different conformational states of UHRF1 and NP95 
(A) Schematic representation of UHRF1 binding state A, PBR is interacting with the TTD surface, only the PHD domain can interact with the $\mathrm{H} 3$ tail peptide, SRA domain is not interacting with DNA. (B) Schematic representation of UHRF1 binding state $B$, PBR is not interacting with the TTD surface, the TTD and PHD domain can interact with the H3 tail peptide, SRA domain is interacting with DNA. (C) Schematic representation of NP95 I binding state, TTD-PHD linker is interacting with the TTD surface, only the PHD domain can interact with the H3 tail peptide, SRA domain is interacting with DNA. (D) Schematic representation of NP95 II binding state, TTD-PHD linker is interacting with the TTD surface, the TTD and PHD domain can interact with the $\mathrm{H} 3$ tail peptide, SRA domain is interacting with DNA.

\subsubsection{Inter-domain linker regions improve binding specificity for multivalent targets}

In presence of high local concentrations of the H3K9me3 tail at heterochromatic loci, the simultaneous interaction of H3K9me3 and TTD-PHD linker could overcome the PBR dependent blocking of the TTD. Therefore, the interplay of the different inter-domain linker regions on the TTD surface might be a mechanism to increase the specificity of UHRF1 for the modification. Thus, it prevents incorrect readout of histone marks by UHRF1. Similar effects were already shown for the Nck multidomain adapter protein. Similar to UHRF1, the binding specificity of the protein is regulated by the interplay of two linker regions (Takeuchi et al., 2010). This interplay improved the specificity of the protein and as a result it favored bivalent target sites.

Since NP95 I also shows blocking of the TTD binding to H3K9me3 in vitro, similar mechanisms could exist in this isoform of NP95. Nevertheless, the exact regulation of NP95 I binding specificity remains elusive and might involve secondary interactions targeting the TTD-PHD linker region.

\subsection{Linker influence the nuclear localization of UHRF1/NP95}

\subsubsection{Similar cellular roles of UHRF1 and NP95 I}

Many studies have emphasized the important role of UHRF1 and NP95 I in the maintenance of DNA methylation and other heterochromatic marks during continuous cell divisions (Bronner et al., 2010; Cartron et al., 2013; Jin et al., 2010). UHRF1 and NP95 I were both shown to be involved in DNMT1 recruitment to replicating loci. However, it was also found that UHRF1 binds to promoter regions of euchromatic genes and regulates their expression (Rajakumara et al., 2011; Yao et al., 2016). Some of these loci lack H3K9me3 but exhibit DNA methylation paired with $\mathrm{H} 3$ unmodified at the N-terminus (i.e. absence of H3R2 symmetric dimethylation) (Rajakumara et al., 2011). It is conceivable that UHRF1 binds to these genomic regions by multivalent interaction of the PHD and 
SRA domains with the unmodified N-terminal H3-tail and methylated DNA. NP95 I exhibits a similar binding mode. It was shown to bind to methylated DNA and the Nterminus of $\mathrm{H3}$ in vitro, while it was not binding to H3K9me3. Therefore, we expect that NP95 I might be recruited to such loci in the cell, comparable to UHRF1.

It was found that NP95 I interacts with the de novo DNA methyltransferases DNMT3a and DNMT3b and that this complex mediates epigenetic silencing in a histone H3 lysine 9 methyltransferase (i.e. G9a, Suv39h1/2) dependent manner (Meilinger et al., 2009). Depletion of NP95 in mouse embryonic stem cells results in severe global DNA hypomethylation (Sharif et al., 2007). A similar DNA methylation defect is caused by depletion of all three DNMTs (Tsumura et al., 2006) or of histone methyltransferases (G9a, Suv39h1/2) (Dong et al., 2008; Lehnertz et al., 2003). Hence, there is circumstantial evidence that NP95 I is not only involved in replication-coupled maintenance of DNA methylation, but also in de novo DNA methylation in an H3K9me3-dependent manner. This was so far not reported for UHRF1.

Others and we showed that UHRF1 and NP95 I were very highly abundant in thymus and intestine (Chapter 3.3.2) (Hopfner et al., 2000). Thymus and intestine are important parts of the human and murine immune system and an important site of T-lymphocyte proliferation and maturation. A key process of T-cell maturation is $\mathrm{V}(\mathrm{D}) \mathrm{J}$ recombination, that was shown to involve reorganization of heterochromatic regions (Proudhon et al., 2015; Su et al., 2004). Recent reports reveal a central role of UHRF1 in transcriptional regulation of the macrophage migration inhibitory factor in monocytes, B- and Tlymphocytes and the importance of NP95 in maturation of colonic regulatory T-cells (Obata et al., 2014; Yao et al., 2016). The high similarity of expression levels of NP95 I and UHRF1 in these tissues and the very low abundance of NP95 II mRNA in the murine intestine, suggests that immune system related functions of UHRF1 in human might be resumed by NP95 I in mouse.

\subsubsection{Potential cellular role of NP95 II}

The expression of NP95 II mRNA is much lower than NP95 I mRNA. NP95 II shows highest expression in adult ovary, thymus and duodenum, suggesting an important function in these tissues. In this study I demonstrated that the protein establishes a multivalent binding mode to a $\mathrm{H} 3$ tail with an unmodified N-terminus and $\mathrm{K} 9 \mathrm{me} 3$ comparable to the UHRF1 B state (Figure 4-1B, D) (Arita et al., 2012; Cheng et al., 2013; Rothbart et al., 2013; Xie et al., 2012). While the functions of UHRF1 and NP95 I are extensively studied, there is nothing known about the NP95 II isoform of the protein. In this study, I show that NP95 II TTD binding to H3K9me3 is neither blocked by the PBR 
nor by the TTD-PHD linker. In contrast to UHRF1 the protein lost the consensus recognition motif of PKA (R-X-X-S) in the TTD-PHD linker. Therefore, I think this isoform adopts a constant multivalent binding mode and targets a special subset of loci. The nature of NP95 II action in vivo will be the subject of future experiments.

\subsection{Regulation of the TTD interaction by different inter-domain linker regions}

In cells, UHRF1 and NP95 are localized to pericentromeric heterochromatin in the S-phase of the cell cycle (Bostick et al., 2007; Miura et al., 2001; Papait et al., 2007; Sharif et al., 2007). Pericentromeric heterochromatin is generally thought to be rich in DNAmethylation and H3K9me3 (reviewed in (Dejardin, 2015)). It was previously reported that the TTD, PHD and SRA domains are all participating in targeting of UHRF1 and NP95 I to heterochromatin (Liu et al., 2013; Rothbart et al., 2013; Rottach et al., 2010).

I show that in NIH3T3 cells UHRF1 localization to this region is dependent on the domains that bind these modifications, the SRA and the TTD domain. In vitro, the C-terminal PBR region blocks the interaction of the TTD with H3K9me3 (Gelato et al., 2014). Therefore we thought that TTD/PBR interaction influences the nuclear localization of the protein. Indeed, overexpression of the full-length protein together with the PBRRING region impaired co-localization with the pericentromeric heterochromatin while a mutant PBR-RING did not (Gelato et al., 2014).

In NP95 I, we found a similar linker dependent mechanism to block the TTD aromatic cage and thus impair the interaction with $\mathrm{H} 3 \mathrm{~K} 9 \mathrm{me}$. It was described earlier that the TTD is essentially contributing to the co-localization of NP95 I with pericentromeric heterochromatin (Rottach et al., 2010). Given the importance of the TTD in the nuclear localization of UHRF1 as well as NP95 I, it is crucial to tightly control the TTD interactions with the different inter-domain linker regions in UHRF1 as well as NP95.

In agreement with former studies I show that the nuclear localization of UHRF1 to pericentromeric heterochromatin is also dependent on the interaction of the SRA domain with hemimethylated DNA (Gelato et al., 2014; Liu et al., 2013). Similar dependency on the SRA domain was demonstrated for NP95 (Rottach et al., 2010). Hence, UHRF1 as well as NP95 need the combined read out of DNA-methylation and H3K9me3 to faithfully localize to pericentromeric heterochromatin in the nucleus. It was already reported that the SRA domain alone does not co-localize with full-length protein in cells (Rottach et al., 2010). Therefore, altering binding contribution of the TTD caused by intramolecular interactions with linker regions could vary the target specificity of the protein. Regulatory 102 
processes affecting the TTD/linker interaction might give rise to target site variation that can be important to fulfill the multitude of reported functions of UHRF1/NP95 in replication, transcriptional regulation, DNA-damage response and immune cell maturation.

\subsubsection{Characteristic features of linker regulations}

While intrinsically disordered, the linker regions might nevertheless adopt a series of limited, defined transitional states enabling interaction with different polar surfaces of UHRF1/NP95 and determining different conformational and binding states. The kinetic barrier for these transitions could be relatively low due to the intrinsic linker flexibility. As consequence, the protein might be able to rapidly change conformations in response to external stimuli and structural perturbations. This in turn would facilitate changes in the population of certain states therefore affecting UHRF1/NP95 target binding and cellular function (Ma et al., 2011; Tauber and Fischle, 2015).

Sequence comparison of the linker regions regulating the binding behavior of UHRF1 and NP95 highlights the common characteristic features of regulatory linkers. All linker sequences comprise a $\mathrm{K} / \mathrm{R}$-rich basic patch or at least an essential basic residue, which is closely accompanied by a threonine or serine residue. The NP95 I TTD-PHD linker contains an additional Proline-rich region. All these linker regions interact with the surface of the TTD domain. This domain is formed by antiparallel $\beta$-sheets and contains a hydrophobic ligand pocket for the interaction with H3K9me3.

Comparable intramolecular interactions of inter-domain linker regions with hydrophobic pocket containing domains are described for Src homology domain proteins (SH2, SH3). These proteins comprise SH3 domains that recognize a solvent exposed P-X-X-P motif (Freund et al., 2008). Comparable with UHRF1 TTD domain, the SH3 domain consists of $\beta$-strands that are arranged in two anti-parallel $\beta$-sheets and contain a hydrophobic ligand pocket. In Nck, a basic patch in a linker region accompanied by a serine residue, establishes interactions ( $\mathrm{Kd}=2 \mathrm{mM}$ ) with a SH3 domain masking its P-X-X-P binding site. This mechanism shows high similarity with the PBR-dependent blocking of the TTD in UHRF1. The Crk signaling protein shows intramolecular interactions of a proline containing linker with the surface of a SH3 domain that are modulating target interaction of the protein. In NP95 I, I revealed that a proline patch in the TTD-PHD linker is essential for blocking of the binding of the TTD to H3K9me3.

In contrast, $\mathrm{SH} 2$ domains recognize phosphorylated tyrosin residues by a phosphate binding loop and an adjacent hydrophobic pocket (Pawson et al., 2001). In Syk, two SH2 
domains are separated by a 23 amino acid linker insertion that is thought to regulate its target interaction. This linker insertion is particularly rich in prolines, serines and basic residues. In UHRF1 and NP95 inter-domain linker regions we found same residues playing important roles in regulation of the binding specificity of the proteins. The similarities we found between linker regulated proteins might also give insights into common regulatory mechanisms to alter multidomain protein target binding. Given the common characteristics of the described mechanisms, these can be used to identify new putative linker regulated proteins.

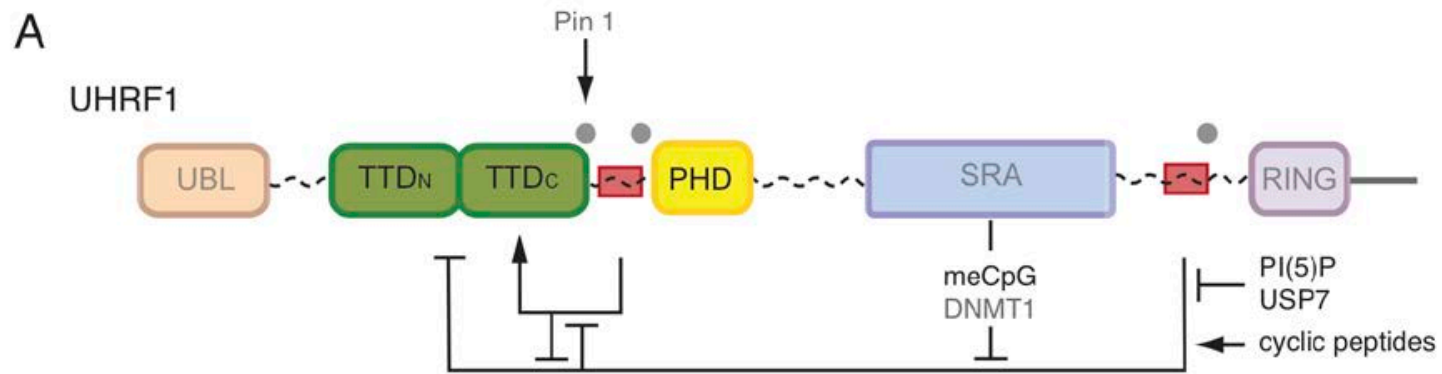

B

NP95 ।

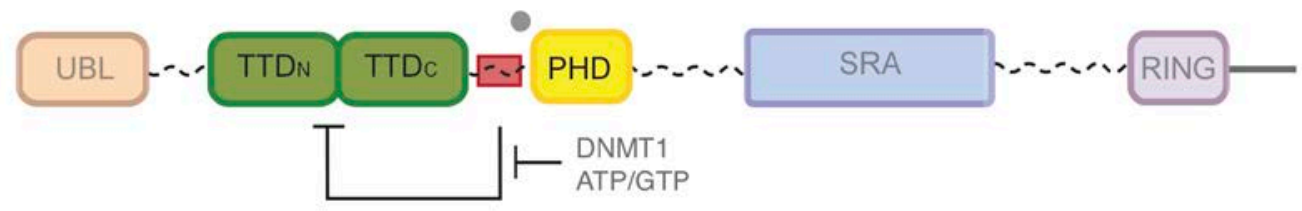

C

NP95 ॥

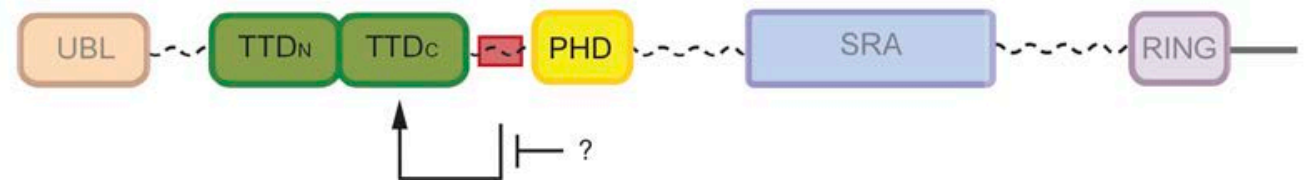

Figure 4-2 Regulation of TTD/ linker interactions

Schematic representation of UHRF1 (A), NP95 I (B) and NP95 II (C) domain organization, highlighted are different regulatory linker regions affecting TTD interaction with H3K9me3, shown are regulatory mechanisms we and others described (black) and putative mechanisms (grey), grey dots show potential sites of post translational phosphorylation.

\subsubsection{Small molecule ligands:}

Previous work of our laboratory identified PI(5)P as a small molecule regulator of UHRF1 TTD binding to H3K9me3, by interacting with the PBR region (Gelato et al., 2014). While we do not yet know how exactly PI(5)P is bound by UHRF1. It is interesting to note that the PBR resembles in sequence a previously defined consensus PIP interaction motif $\left(\mathrm{R} / \mathrm{K}-\left(\mathrm{X}_{\mathrm{n}=3-7}\right)-\mathrm{K}-\mathrm{X}-\mathrm{K} / \mathrm{R}-\mathrm{K} / \mathrm{R}\right)$ shared by several PIP interacting factors (Lewis et al., 2011). 
In this study, I show that manipulation of cellular PI5P levels affects UHRF1 subnuclear localization. Several links between the reported functions of UHRF1 and nuclear PI(5)P levels support the idea of allosteric control of the protein. It was shown previously that nuclear PI(5)P levels are tightly regulated during the cell cycle and in response to certain cellular signaling cascades. PI(5)P levels increase up to 20-fold during transition of G1- to S-phase (Clarke et al., 2001; Divecha et al., 1991; Jones et al., 2006; Jones et al., 2013). Cell cycle analysis of UHRF1 localization in relation to heterochromatic foci indicates changed distribution at the onset of S-phase (Papait et al., 2007), which might be caused by regulation of TTD/H3K9me3 interaction by PI(5)P. Also, UHRF1 interacts with several factors associated with the DNA damage response such as Eme1, TIP60, Ku70, and PARP1 (Achour et al., 2009; De Vos et al., 2014; Mistry et al., 2008; Sharif et al., 2007). PI(5)P levels are increased after irradiation and exposure to oxidative stress (Clarke et al., 2001; Divecha et al., 1991; Jones et al., 2006; Jones et al., 2013). Indeed, recent studies revealed a role of UHRF1 in DNA damage response, as a sensor for DNA interstrand crosslinks (Liang et al., 2015; Tian et al., 2015).

The role of PI(5)P in the regulation of UHRF1 binding behavior to H3K9me3 shows the importance of small molecules in timed and site-specific localization of the protein. This led us to investigate the potential of additional small molecule regulators. We identified two potential cyclic peptide regulators of UHRF1, D4 (PYNRILIKRHRVKII) and D5 (PYVLIYVNNHKRPSR) and demonstrated that these peptides interact with the PBRRING region of UHRF1. In addition, the peptides caused a significant increase in the interaction of this region with the TTD-PHD region of the protein. Therefore, the peptides had opposite effects than PI(5)P. The cyclic peptides could thus be a tool to lock UHRF1 in state A (Figure 4-1A) and drastically decrease the interaction of the protein with H3K9me3 and putatively DNA methylation. Potentially, the peptide candidates resulting from our screening procedures might be relevant candidates for future medical approaches to target UHRF1 in cancerous tissues.

In NP95 I, we found the TTD-PHD linker playing an important role in regulation of the binding to H3K9me3. It was proposed that the linker insertion in NP95 I harbors a ATP/GTP-binding site motif between A296 and S303 (Fujimori et al., 1998; Hopfner et al., 2000). It is likely that binding of ATP or GTP to the TTD-PHD linker would impair the interaction between the linker and the TTD. As a result the TTD might be available to bind to H3K9me3 again. Indeed, GTP might be relevant in NP95 I regulation. GTP hydrolysis is essential for cell cycle progression (Avis and Clarke, 1996). NP95 I was shown to be essential progression of the S-phase and its depletion is linked to cell cycle arrest. In contrast, the reduction of intracellular GTP levels results in differentiation or 
even apoptosis (Meshkini and Yazdanparast, 2010). This suggests that NP95 I binding to H3K9me3 could be regulated by this molecule in the cell.

\subsubsection{Posttranslational modification}

The transitions between different conformational states of UHRF1 and NP95 conveyed by the linker regions might be further regulated by posttranslational modifications (PTM). Given the importance of basic patches or residues for the interaction between the linkers and the TTD domain, it is important to note that serine and threonine residues often accompany these. This amino acids are targets for posttranslational phosphorylation. Phosphorylation could impair binding of the linker to the peptide-binding groove of the TTD or putatively other regions of the protein by impairing their electrostatic interactions. Indeed, for UHRF1 it was shown previously that phosphorylation of a serine residue (S298) adjacent to R295-R296-K297 by PKA, decreases the binding affinity of the TTDPHD cassette for the H3K9me3-tail. The altered binding stoichiometry of the complex indicates that the coupling of TTD and PHD mediated by TTD-PHD linker is impaired by the PTM (Arita et al., 2012). Interestingly, the PKA consensus motif (R-X-X-S) is neither conserved in NP95 I nor NP95 II suggesting that the murine proteins are not phosphorylated at that position.

A number of additional sites of serine phosphorylation and other posttranslational modifications have been identified in UHRF1 as well as NP95 by proteomics studies. Some of these map to highly conserved residues within the TTD-PHD linker and the PBR region (Dephoure et al., 2008; Ma et al., 2012; Olsen et al., 2006; Rigbolt et al., 2011). These events have the potential to regulate the proteins conformational states. In UHRF1 for example, phosphorylation of S651 that is found during human embryonic stem cell differentiation and mitosis, is next to the K648-R649-K650 element (Ma et al., 2012; Rigbolt et al., 2011). This might impair the interaction of the PBR with the peptide-binding groove of the TTD thereby enhancing a particular UHRF1 chromatin-binding mode.

Similar effects of posttranslational phosphorylation were predicted for the linker dependent regulation of Nck and have been reported for Syk (Paris et al., 2010; Takeuchi et al., 2010). Syk is a target of PKC in cells and is phosphorylated at a serine residue in the center of the regulatory linker. Interestingly, the 9 amino acid insertion in the NP95 I TTDPHD linker matches the general PKC consensus motif $(R / K)_{1-3}-(X)_{0-2}-T / S-(X)_{0-2}-(R / K)_{1-3}$ generating a putative binding site for PKC (Kennelly and Krebs, 1991). Therefore, NP95 I is a potential substrate and might get phosphorylated on a threonine residue (T300) located between R298 and K302. This phosphorylation could potentially impair the interaction of R298 with the TTD surface and enable the binding to H3K9me3. 


\subsubsection{Prolyl isomerization}

In addition to the discussed PTMs, serine S287 of UHRF1 is posttranslationally phosphorylated during T-cell receptor signaling, mitosis and human embryonic stem cell differentiation (Olsen et al., 2006; Rigbolt et al., 2011). The same phosphorylation was reported in rat UHRF1 and might also exist in the murine protein (Lundby et al., 2012). This phosphorylation site is C-terminally accompanied by a proline residue (P288) that might be a target for Pin1 directed prolyl isomerization (Figure 4-2). Pin1 is known to specifically target the proline residues that are accompanied by a phosphorylated serine residue. It catalyzes cis-trans isomerization of proline imidic peptide bonds exclusively on phosphorylated target sites (Rostam et al., 2015). In UHRF1/NP95, this isomerization could impair or change the positioning of the TTD-PHD linker on the TTD surface and thus the multivalent binding of TTD and PHD in a phosphorylation signal dependent manner. Similar to UHRF1 and NP95, Pin1 is important for cell cycle progression between $\mathrm{G}_{1} / \mathrm{G}_{0^{-}}$and S-phase and plays a key role in cellular signal transduction pathways (Rostam et al., 2015). This might be a versatile mechanism to regulate the target binding specificity of the protein during cell cycle or cellular differentiation processes.

It has been shown that isomerization of proline bonds can tune the binding affinity of a protein. In MLL1 a proline in the linker between the PHD3 and the bromodomain is subject of СyP33 dependent prolyl isomerization that changes the relative spacing of the two domains. This results in a reduced affinity for its target H3K4me2/3 as well as the generation of a new interaction interface of the PHD3 domain (Wang et al., 2010). The relevance of proline switches for linker dependent regulation is also demonstrated for the SH3 containing Crk signaling protein (Sarkar et al., 2011). In Crk the cyclophilin Adependent proline cis-trans isomerization in a regulatory linker region affects target site specificity of the protein by releasing novel $\mathrm{SH} 3$ binding surfaces.

\subsubsection{Protein-protein interactions}

UHRF1/NP95 is known to interact with different proteins such as DNMTs, G9a and USP7. These interactions require distinct, specific surfaces that will or will not be available in the different conformational states of UHRF1/NP95. Conversely, it is expected that engagement of a particular protein partner will stabilize a certain conformation and therefore a certain chromatin mark-binding mode of UHRF1 or NP95. USP7 was recently found to interact with the SRA-RING linker region of UHRF1 engaging lysine residues K646 and K648 (Ma et al., 2012; Zhang et al., 2015). These lysines have also been shown to be essential for the TTD/ PBR interaction in human UHRF1 (Gelato et al., 2014). The 
interaction with USP7 was shown to affect the TTD/PBR interaction in vitro as well as the UHRF1 recruitment to chromatin in vivo. It specifically interacts with UHRF1 in S-phase of the cell cycle and prevents its ubiquitinylation. In turn, mitotic phosphorylation at S651 of UHRF1 dissociates the deubiquitylase from the PBR (Ma et al., 2012). Interestingly, the identified recognition motif of USP7 in the SRA-RING linker is also conserved in NP95 (Zhang et al., 2015). Therefore, we expect similar interactions of USP7 with NP95. Since the TTD/PBR interaction is not conserved in NP95, the association of USP7 with the protein should not affect the binding of the TTD to H3K9me3. Nevertheless, TTD domain binding to this modification was shown to be essential for the recruitment of the protein to pericentromeric heterochromatin (Rottach et al., 2010).

In this study we showed that UHRF1 SRA domain interaction with hemimethylated DNA enhances the interaction of the TTD with the H3K9me3 peptide, thus is impairing the PBR dependent blocking of the TTD aromatic cage. It is reported that the SRA is not only interacting with DNA, but is also establishing strong connections to DNMT1 (Achour et al., 2009; Bashtrykov et al., 2014). I reasoned that this interaction might influence the TTD / PBR interaction in UHRF1. While DNMT1 was shown to only interact with the SRA domain in UHRF1, a second interaction site of the protein was reported in NP95 I (Achour et al., 2009; Bashtrykov et al., 2014; Berkyurek et al., 2014; Bostick et al., 2007). This binding site of DNMT1 was mapped to the region of the TTD-PHD linker and the PHD domain of NP95 I (Bostick et al., 2007). Since we have shown here that the TTD-PHD linker is blocking the TTD interaction with H3K9me3, the interaction of DNMT1 with this region might be a regulatory mechanism to release this block. It is known that the TTD domain interaction with $\mathrm{H} 3 \mathrm{~K} 9 \mathrm{me} 3$ is essential for proper localization of NP95 I to pericentromeric heterochromatin in vivo (Rottach et al., 2010). Therefore the binding of DNMT1 to the TTD-PHD linker might facilitate proper localization of the protein in vivo.

The linker insertion in NP95 I comprises other features to putatively regulate the TTD/TTD-PHD linker interactions. proline-rich motifs (PRMs) are important hubs of protein-protein interactions that are bound by PRM recognizing domains (PRD) (Freund et al., 2008). The linker insert of NP95 I is especially rich in proline residues, which could be important for interactions with PRD comprising proteins. These proteins play key roles in a variety of cellular signaling events and their interaction with the proline patch could impair the TTD/ TTD-PHD linker interaction in NP95 I. This could regulate the NP95 I chromatin association, similar to how USP7 regulates UHRF1. 


\subsection{Alternative splicing of linker regions}

In mice, alternative splicing generates two isoforms of UHRF1. The difference of only nine amino acids between the isoforms give rise to two proteins that substantially differ in the binding behavior regarding $\mathrm{K} 9$ trimethylation of the $\mathrm{H} 3$ tail. The linker insertion introduces residues in the TTD-PHD linker that establish interactions with the TTD, blocking the H3K9me3 binding. At the same time NP95 II is able to establish a multivalent binding mode of TTD and PHD that was not shown for NP95 I. Since the two splicing variants of NP95 resemble the two binding states we found for UHRF1, the target interaction of the murine protein could also be regulated on a transcriptional level. How the differential splicing events are regulated during the cell cycle and cell differentiation, however, remains to be elucidated.

I found notable similarities between the inter-domain linker dependent regulatory mechanisms in UHRF1/NP95 and the proteins of the Src homology domain proteins. Syk is a member of this protein family, resembling the described key features of the linker dependent regulation (Chapter 4.3.1). Similar to NP95, the Syk family of tyrosine protein kinases exemplifies the importance of alternative splicing events in inter-domain linker sequences. Alternative splicing events give rise to two different splicing variants of Syk proteins, Syk and SykB. The proteins only differ by a 23 amino acid insertion in the linker between the tandem $\mathrm{SH} 2$ domains and the catalytic domain of the proteins. This insertion is highly conserved in human, rat and mouse. As a consequence of the insertion, Syk exhibits a higher target affinity for phosphorylated immunoreceptor tyrosine-based activation motifs and is therefore much more efficiently mediates the immunoreceptor signaling (Latour et al., 1998). I found a similar alternative splicing event regulating the binding affinity of NP95. Additionally, the insertion found in NP95 I harbors putative regulatory features not found in NP95 II. Similar to Syk, the linker insert in NP95 I comprises a PKC binding motif. As described above, Syk function is modulated by PKC dependent phosphorylation, while SykB is not.

Different isoforms are expressed from the same gene using specific alternative splicing mechanisms dependent on tissue or environmental conditions. Since the same coding sequence can be used to produce different proteins that fulfill different cellular functions, this mechanism improves the coding capacity of the genome. I reasoned that the alternative splicing events in inter-domain linker regions might in general give rise to differentially regulated multidomain proteins. In this way, proteins with slight variations in target specificity can be expressed. These different variants can be recruited to differentially modified loci and affect chromatin structure or function. The alternatively 
spliced linker regions might also carry different regulatory features. Thus proteins could be targeted to specific loci in response to different external stimuli.

\subsection{Using small molecules to target UHRF1 in cancer}

UHRF1 is found in a complex with enzymes catalyzing histone and DNA modifications that are mainly located in transcriptionally inactive chromatin (reviewed in (Almouzni and Probst, 2011; Bernstein et al., 2007; Dejardin, 2015), such as HDAC1, DNMT1 and G9a (Cartron et al., 2013; Jin et al., 2010), where it targets these catalytic proteins to heterochromatic loci using the TTD and SRA domain (Nady et al., 2011; Unoki et al., 2004). UHRF1 is a central component of this complex ensuring the faithful copy of silencing epigenetic marks during replication, preserving gene repression (Bronner et al., 2010; Cartron et al., 2013; Jin et al., 2010).

UHRF1 expression was found to be aberrant in cancer cells (Mousli et al., 2003). Elevated levels of UHRF1 expression have been found in many human cancerous tissues including bladder, breast, cervix, kidney, lung, pancreas and prostate (Bronner et al., 2007; Unoki et al., 2009; Unoki et al., 2010). The high levels of the protein result in loss of contact inhibition and hypermethylation in tumor suppressor genes (Alhosin et al., 2011; Daskalos et al., 2011; Hopfner et al., 2002; Sabatino et al., 2012). The down regulation of UHRF1 in this context was shown to give rise to re-expression of tumor suppressors, cell cycle arrest and apoptosis (Alhosin et al., 2011; Jeanblanc et al., 2005; Qin et al., 2015; Tien et al., 2011). Therefore, UHRF1 is a versatile target for anti-cancer therapy in many tissues (Alhosin et al., 2011; Unoki et al., 2009). It was already proposed that small molecule compounds could be used to impair SRA binding to hemimethylated DNA and to reduce UHRF1-dependent hypermethylation at tumor suppressor genes (Unoki et al., 2009). A recent report has revealed small molecules that modify the binding of the domain to DNA, leading to inhibition of DNA-methylation in vitro (Myrianthopoulos et al., 2016). However targeting the SRA domain of UHRF1 is not an ideal approach due to the fact that UHRF2, another protein of the same family, comprises a similar domain. In contrast, the TTD/PBR interaction we described is UHRF1 specific (Gelato et al., 2014) and can be used to impair the interaction of the protein with its target sites. In this study we could show that UHRF1 binding to H3K9me3 and DNA methylation are not separate events but are closely interconnected. Small molecules targeting the TTD/PBR interaction will thus affect both binding events. In our cyclic peptide screening we found that D4 and D5 peptides are promising small molecule regulators of UHRF1 and could be used to target the protein in cancerous tissues. 


\section{Conclusion}

Ubiquitin-like with PHD and RING Finger domains 1 (UHRF1 in human, NP95 in mouse) is an important epigenetic regulator connected to apoptosis and tumor development. UHRF1/NP95 integrates different chromatin modifications and is essential for their maintenance throughout the cell cycle. UHRF1/NP95 comprises domains for interaction with histones of different modification states (TTD and PHD) and methylated DNA (SRA) coupled to a catalytically active E3 Ubiquitin ligase domain (RING). While multivalent binding of multidomain proteins to complex patterns of chromatin modifications is a hallmark of the histone code, the regulation of such factors has not been fully investigated. Emerging evidence indicates that the chromatin binding modules of UHRF1/ NP95 do not act in isolation but in concert to orchestrate coordinated interaction with different histone and DNA modifications. Here, we dissected the molecular details of regulation of chromatin binding by UHRF1/NP95. Using intramolecular crosslinking analysis and NMR studies we show that flexible inter-domain linker regions establish contacts between the different chromatin-binding modules and globally direct the overall folding and spatial domain arrangement of the protein. These contacts are modulated by ligand binding (histones, DNA) as well as by the allosteric regulator phosphatidylinositol 5-phosphate (PI5P). Indeed, in vitro binding experiments imply that the unstructured linker regions of the protein can alter binding behavior of the chromatin interaction domains and therefore control specificity of UHRF1/NP95. Interestingly, we found that murine NP95 has two splice variants, which only differ in a nine amino acid insertion in one of the inter-domain linker regions. In agreement with our hypothesis, this insertion is sufficient to facilitate differential regulation of chromatin binding by the two isoforms.

Overall, our results show that flexible inter-domain linkers are versatile tools to dynamically regulate the interplay of several domains. We therefore propose a general model of fine-tuned combinatorial readout of epigenetic modifications by certain multidomain proteins. 


\section{References}

Achour, M., Fuhrmann, G., Alhosin, M., Ronde, P., Chataigneau, T., Mousli, M., SchiniKerth, V.B., and Bronner, C. (2009). UHRF1 recruits the histone acetyltransferase Tip60 and controls its expression and activity. Biochem Biophys Res Commun 390, 523-528.

Alhosin, M., Sharif, T., Mousli, M., Etienne-Selloum, N., Fuhrmann, G., Schini-Kerth, V.B., and Bronner, C. (2011). Down-regulation of UHRF1, associated with re-expression of tumor suppressor genes, is a common feature of natural compounds exhibiting anticancer properties. J Exp Clin Cancer Res 30, 41.

Allan, J., Mitchell, T., Harborne, N., Bohm, L., and Crane-Robinson, C. (1986). Roles of H1 domains in determining higher order chromatin structure and H1 location. J Mol Biol 187, 591-601.

Allis, C.D., Jenuwein, T., Reinberg, D., and Caparros, M.-L. (2007). Epigenetics.

Almouzni, G., and Probst, A.V. (2011). Heterochromatin maintenance and establishment: lessons from the mouse pericentromere. Nucleus 2, 332-338.

Alvarez-Venegas, R., Sadder, M., Hlavacka, A., Baluska, F., Xia, Y., Lu, G., Firsov, A., Sarath, G., Moriyama, H., Dubrovsky, J.G., et al. (2006). The Arabidopsis homolog of trithorax, ATX1, binds phosphatidylinositol 5-phosphate, and the two regulate a common set of target genes. Proc Natl Acad Sci U S A 103, 6049-6054.

Arima, Y., Hirota, T., Bronner, C., Mousli, M., Fujiwara, T., Niwa, S., Ishikawa, H., and Saya, H. (2004). Down-regulation of nuclear protein ICBP90 by p53/p21Cip1/WAF1dependent DNA-damage checkpoint signals contributes to cell cycle arrest at G1/S transition. Genes Cells 9, 131-142.

Arita, K., Isogai, S., Oda, T., Unoki, M., Sugita, K., Sekiyama, N., Kuwata, K., Hamamoto, R., Tochio, H., Sato, M., et al. (2012). Recognition of modification status on a histone H3 tail by linked histone reader modules of the epigenetic regulator UHRF1. Proc Natl Acad Sci U S A 109, 12950-12955.

Ausio, J., Dong, F., and van Holde, K.E. (1989). Use of selectively trypsinized nucleosome core particles to analyze the role of the histone "tails" in the stabilization of the nucleosome. J Mol Biol 206, 451-463.

Avis, J.M., and Clarke, P.R. (1996). Ran, a GTPase involved in nuclear processes: its regulators and effectors. J Cell Sci 109 ( Pt 10), 2423-2427.

Avvakumov, G.V., Walker, J.R., Xue, S., Li, Y., Duan, S., Bronner, C., Arrowsmith, C.H., and Dhe-Paganon, S. (2008). Structural basis for recognition of hemi-methylated DNA by the SRA domain of human UHRF1. Nature 455, 822-825. 
Balla, T., Wymann, M., and York, J.D. (2012). Phosphoinositides II: The Diverse Biological Functions, Vol 59 (Dordrecht: Springer Netherlands).

Barlow, C.A., Laishram, R.S., and Anderson, R.A. (2010). Nuclear phosphoinositides: a signaling enigma wrapped in a compartmental conundrum. Trends Cell Biol 20, 25-35.

Bartke, T., Vermeulen, M., Xhemalce, B., Robson, S.C., Mann, M., and Kouzarides, T. (2010). Nucleosome-interacting proteins regulated by DNA and histone methylation. Cell $143,470-484$.

Bashtrykov, P., Jankevicius, G., Jurkowska, R.Z., Ragozin, S., and Jeltsch, A. (2014). The UHRF1 protein stimulates the activity and specificity of the maintenance DNA methyltransferase DNMT1 by an allosteric mechanism. J Biol Chem 289, 4106-4115.

Berkyurek, A.C., Suetake, I., Arita, K., Takeshita, K., Nakagawa, A., Shirakawa, M., and Tajima, S. (2014). The DNA methyltransferase Dnmt1 directly interacts with the SET and RING finger-associated (SRA) domain of the multifunctional protein Uhrf1 to facilitate accession of the catalytic center to hemi-methylated DNA. J Biol Chem 289, 379-386.

Bernstein, B.E., Meissner, A., and Lander, E.S. (2007). The mammalian epigenome. Cell $128,669-681$.

Bestor, T.H., Edwards, J.R., and Boulard, M. (2015). Notes on the role of dynamic DNA methylation in mammalian development. Proc Natl Acad Sci U S A 112, 6796-6799.

Bird, A., Tate, P., Nan, X., Campoy, J., Meehan, R., Cross, S., Tweedie, S., Charlton, J., and Macleod, D. (1995). Studies of DNA methylation in animals. J Cell Sci Suppl 19, 37-39.

Birnboim, H.C., and Doly, J. (1979). A rapid alkaline extraction procedure for screening recombinant plasmid DNA. Nucleic Acids Res 7, 1513-1523.

Bohm, L., and Crane-Robinson, C. (1984). Proteases as structural probes for chromatin: the domain structure of histones. Biosci Rep 4, 365-386.

Bostick, M., Kim, J.K., Esteve, P.O., Clark, A., Pradhan, S., and Jacobsen, S.E. (2007). UHRF1 plays a role in maintaining DNA methylation in mammalian cells. Science 317 , 1760-1764.

Boule, J.B., Mozziconacci, J., and Lavelle, C. (2015). The polymorphisms of the chromatin fiber. J Phys Condens Matter 27, 033101.

Breiling, A., and Lyko, F. (2015). Epigenetic regulatory functions of DNA modifications: 5methylcytosine and beyond. Epigenetics Chromatin 8, 24.

Bronner, C., Achour, M., Arima, Y., Chataigneau, T., Saya, H., and Schini-Kerth, V.B. (2007). The UHRF family: oncogenes that are drugable targets for cancer therapy in the near future? Pharmacol Ther 115, 419-434. 
Bronner, C., Fuhrmann, G., Chedin, F.L., Macaluso, M., and Dhe-Paganon, S. (2010). UHRF1 Links the Histone code and DNA Methylation to ensure Faithful Epigenetic Memory Inheritance. Genet Epigenet 2009, 29-36.

Brown, S.W. (1966). Heterochromatin. Science 151, 417-425.

Bruce Alberts, A.J., Julian Lewis, Martin Raff, Keith Roberts, Peter Walter (2007). Molecular Biology of the Cell, 5th edition

Bultsma, Y., Keune, W.J., and Divecha, N. (2010). PIP4Kbeta interacts with and modulates nuclear localization of the high-activity PtdIns5P-4-kinase isoform PIP4Kalpha. Biochem J 430, 223-235.

Burnette, W.N. (1981). "Western blotting": electrophoretic transfer of proteins from sodium dodecyl sulfate--polyacrylamide gels to unmodified nitrocellulose and radiographic detection with antibody and radioiodinated protein A. Anal Biochem 112, 195-203.

Cartron, P.F., Blanquart, C., Hervouet, E., Gregoire, M., and Vallette, F.M. (2013). HDAC1mSin3a-NCOR1, Dnmt3b-HDAC1-Egr1 and Dnmt1-PCNA-UHRF1-G9a regulate the NYESO1 gene expression. Mol Oncol 7, 452-463.

Cheng, J., Yang, Y., Fang, J., Xiao, J., Zhu, T., Chen, F., Wang, P., Li, Z., Yang, H., and Xu, Y. (2013). Structural insight into coordinated recognition of trimethylated histone H3 lysine 9 (H3K9me3) by the plant homeodomain (PHD) and tandem tudor domain (TTD) of UHRF1 (ubiquitin-like, containing PHD and RING finger domains, 1) protein. J Biol Chem 288, 1329-1339.

Chu, J., Loughlin, E.A., Gaur, N.A., SenBanerjee, S., Jacob, V., Monson, C., Kent, B., Oranu, A., Ding, Y., Ukomadu, C., et al. (2012). UHRF1 phosphorylation by cyclin A2/cyclindependent kinase 2 is required for zebrafish embryogenesis. Molecular biology of the cell 23, 59-70.

Clarke, J.H., Letcher, A.J., D'Santos C, S., Halstead, J.R., Irvine, R.F., and Divecha, N. (2001). Inositol lipids are regulated during cell cycle progression in the nuclei of murine erythroleukaemia cells. Biochem J 357, 905-910.

Cooper, G.M., and Hausman, R.E. (2000). The cell (Sinauer Associates Sunderland).

Cuthbert, G.L., Daujat, S., Snowden, A.W., Erdjument-Bromage, H., Hagiwara, T., Yamada, M., Schneider, R., Gregory, P.D., Tempst, P., Bannister, A.J., et al. (2004). Histone deimination antagonizes arginine methylation. Cell 118, 545-553.

Cutter, A.R., and Hayes, J.J. (2015). A brief review of nucleosome structure. FEBS Lett 589, 2914-2922. 
Dagert, M., and Ehrlich, S.D. (1979). Prolonged incubation in calcium chloride improves the competence of Escherichia coli cells. Gene 6, 23-28.

Daskalos, A., Oleksiewicz, U., Filia, A., Nikolaidis, G., Xinarianos, G., Gosney, J.R., Malliri, A., Field, J.K., and Liloglou, T. (2011). UHRF1-mediated tumor suppressor gene inactivation in nonsmall cell lung cancer. Cancer 117, 1027-1037.

Davey, C.A., Sargent, D.F., Luger, K., Maeder, A.W., and Richmond, T.J. (2002). Solvent mediated interactions in the structure of the nucleosome core particle at 1.9 a resolution. $\mathrm{J}$ Mol Biol 319, 1097-1113.

De Vos, M., El Ramy, R., Quénet, D., Wolf, P., Spada, F., Magroun, N., Babbio, F., Schreiber, V., Leonhardt, H., and Bonapace, I.M. (2014). Poly (ADP-ribose) polymerase 1 (PARP1) associates with E3 ubiquitin-protein ligase UHRF1 and modulates UHRF1 biological functions. Journal of Biological Chemistry 289, 16223-16238.

Dejardin, J. (2015). Switching between Epigenetic States at Pericentromeric Heterochromatin. Trends Genet 31, 661-672.

Dephoure, N., Zhou, C., Villen, J., Beausoleil, S.A., Bakalarski, C.E., Elledge, S.J., and Gygi, S.P. (2008). A quantitative atlas of mitotic phosphorylation. Proc Natl Acad Sci U S A 105, 10762-10767.

Dileep, V., Rivera-Mulia, J.C., Sima, J., and Gilbert, D.M. (2015). Large-Scale Chromatin Structure-Function Relationships during the Cell Cycle and Development: Insights from Replication Timing. Cold Spring Harb Symp Quant Biol.

Dillon, N., and Festenstein, R. (2002). Unravelling heterochromatin: competition between positive and negative factors regulates accessibility. Trends Genet 18, 252-258.

Divecha, N., Banfic, H., and Irvine, R.F. (1991). The polyphosphoinositide cycle exists in the nuclei of Swiss 3T3 cells under the control of a receptor (for IGF-I) in the plasma membrane, and stimulation of the cycle increases nuclear diacylglycerol and apparently induces translocation of protein kinase $C$ to the nucleus. The EMBO journal 10, 3207-3214.

Dong, K.B., Maksakova, I.A., Mohn, F., Leung, D., Appanah, R., Lee, S., Yang, H.W., Lam, L.L., Mager, D.L., Schubeler, D., et al. (2008). DNA methylation in ES cells requires the lysine methyltransferase G9a but not its catalytic activity. The EMBO journal 27, 26912701.

Du, J., and Patel, D.J. (2014). Structural biology-based insights into combinatorial readout and crosstalk among epigenetic marks. Biochim Biophys Acta 1839, 719-727.

Eymery, A., Callanan, M., and Vourc'h, C. (2009). The secret message of heterochromatin: new insights into the mechanisms and function of centromeric and pericentric repeat sequence transcription. Int J Dev Biol 53, 259-268. 
Feil, R., and Berger, F. (2007). Convergent evolution of genomic imprinting in plants and mammals. Trends Genet 23, 192-199.

Felsenfeld, G., and Groudine, M. (2003). Controlling the double helix. Nature 421, 448-453.

Fischle, W., Wang, Y., and Allis, C.D. (2003). Binary switches and modification cassettes in histone biology and beyond. Nature 425, 475-479.

Freund, C., Schmalz, H.G., Sticht, J., and Kuhne, R. (2008). Proline-rich sequence recognition domains (PRD): ligands, function and inhibition. Handb Exp Pharmacol, 407429.

Fujimori, A., Matsuda, Y., Takemoto, Y., Hashimoto, Y., Kubo, E., Araki, R., Fukumura, R., Mita, K., Tatsumi, K., and Muto, M. (1998). Cloning and mapping of Np95 gene which encodes a novel nuclear protein associated with cell proliferation. Mamm Genome 9, 1032-1035.

Gallagher, S.R. (2007). One-dimensional SDS gel electrophoresis of proteins. Curr Protoc Cell Biol Chapter 6, Unit 61.

Gasteiger, E., Gattiker, A., Hoogland, C., Ivanyi, I., Appel, R.D., and Bairoch, A. (2003). ExPASy: The proteomics server for in-depth protein knowledge and analysis. Nucleic Acids Res 31, 3784-3788.

Gelato, K.A., Tauber, M., Ong, M.S., Winter, S., Hiragami-Hamada, K., Sindlinger, J., Lemak, A., Bultsma, Y., Houliston, S., Schwarzer, D., et al. (2014). Accessibility of Different Histone H3-Binding Domains of UHRF1 Is Allosterically Regulated by Phosphatidylinositol 5-Phosphate. Molecular Cell 54, 905-919.

Gozani, O., Field, S.J., Ferguson, C.G., Ewalt, M., Mahlke, C., Cantley, L.C., Prestwich, G.D., and Yuan, J. (2005). Modification of protein sub-nuclear localization by synthetic phosphoinositides: evidence for nuclear phosphoinositide signaling mechanisms. Adv Enzyme Regul 45, 171-185.

Gozani, O., Karuman, P., Jones, D.R., Ivanov, D., Cha, J., Lugovskoy, A.A., Baird, C.L., Zhu, H., Field, S.J., Lessnick, S.L., et al. (2003). The PHD finger of the chromatin-associated protein ING2 functions as a nuclear phosphoinositide receptor. Cell 114, 99-111.

Grewal, S.I., and Jia, S. (2007). Heterochromatin revisited. Nat Rev Genet 8, 35-46.

Grigoryev, S.A., and Woodcock, C.L. (2012). Chromatin organization - the $30 \mathrm{~nm}$ fiber. Exp Cell Res 318, 1448-1455.

Hames, D., and Hooper, N. (2000). Biochemistry (Instant notes).

Hanahan, D. (1983). Studies on transformation of Escherichia coli with plasmids. J Mol Biol 166, 557-580. 
Hansen, J.C. (2002). Conformational dynamics of the chromatin fiber in solution: determinants, mechanisms, and functions. Annu Rev Biophys Biomol Struct 31, 361-392.

Hashimoto, H., Horton, J.R., Zhang, X., and Cheng, X.D. (2009). UHRF1, a modular multidomain protein, regulates replication-coupled crosstalk between DNA methylation and histone modifications. Epigenetics 4, 8-14.

Hassa, P.O., Haenni, S.S., Elser, M., and Hottiger, M.O. (2006). Nuclear ADP-ribosylation reactions in mammalian cells: where are we today and where are we going? Microbiol Mol Biol Rev 70, 789-829.

Hayashi, Y., Morimoto, J., and Suga, H. (2012). In vitro selection of anti-Akt2 thioethermacrocyclic peptides leading to isoform-selective inhibitors. ACS Chem Biol 7, 607-613.

Heitz, E. (1928). Der bilaterale Bau der Geschlechtschromosomen und Autosomen bei Pellia Fabbroniana, P. epiphylla und einigen anderen Jungermanniaceen. Planta 5, 725768.

Holliday, R., and Pugh, J.E. (1975). DNA modification mechanisms and gene activity during development. Science 187, 226-232.

Hopfner, R., Mousli, M., Jeltsch, J.M., Voulgaris, A., Lutz, Y., Marin, C., Bellocq, J.P., Oudet, P., and Bronner, C. (2000). ICBP90, a novel human CCAAT binding protein, involved in the regulation of topoisomerase IIalpha expression. Cancer Res 60, 121-128.

Hopfner, R., Mousli, M., Oudet, P., and Bronner, C. (2002). Overexpression of ICBP90, a novel CCAAT-binding protein, overcomes cell contact inhibition by forcing topoisomerase II alpha expression. Anticancer Res 22, 3165-3170.

Hu, L., Li, Z., Wang, P., Lin, Y., and Xu, Y. (2011). Crystal structure of PHD domain of UHRF1 and insights into recognition of unmodified histone $\mathrm{H} 3$ arginine residue 2. Cell Res 21, 1374-1378.

Jeanblanc, M., Mousli, M., Hopfner, R., Bathami, K., Martinet, N., Abbady, A.Q., Siffert, J.C., Mathieu, E., Muller, C.D., and Bronner, C. (2005). The retinoblastoma gene and its product are targeted by ICBP90: a key mechanism in the G1/S transition during the cell cycle. Oncogene 24, 7337-7345.

Jenuwein, T., and Allis, C.D. (2001). Translating the histone code. Science 293, 1074-1080.

Jin, W., Chen, L., Chen, Y., Xu, S.G., Di, G.H., Yin, W.J., Wu, J., and Shao, Z.M. (2010). UHRF1 is associated with epigenetic silencing of BRCA1 in sporadic breast cancer. Breast Cancer Res Treat 123, 359-373. 
Jones, D.R., Bultsma, Y., Keune, W.J., Halstead, J.R., Elouarrat, D., Mohammed, S., Heck, A.J., D'Santos, C.S., and Divecha, N. (2006). Nuclear PtdIns5P as a transducer of stress signaling: an in vivo role for PIP4Kbeta. Mol Cell 23, 685-695.

Jones, D.R., Foulger, R., Keune, W.J., Bultsma, Y., and Divecha, N. (2013). PtdIns5P is an oxidative stress-induced second messenger that regulates PKB activation. FASEB J 27, 1644-1656.

Joti, Y., Hikima, T., Nishino, Y., Kamada, F., Hihara, S., Takata, H., Ishikawa, T., and Maeshima, K. (2012). Chromosomes without a 30-nm chromatin fiber. Nucleus 3, 404-410.

Karagianni, P., Amazit, L., Qin, J., and Wong, J. (2008). ICBP90, a novel methyl K9 H3 binding protein linking protein ubiquitination with heterochromatin formation. Mol Cell Biol 28, 705-717.

Kennelly, P.J., and Krebs, E.G. (1991). Consensus sequences as substrate specificity determinants for protein kinases and protein phosphatases. J biol Chem 266, 15555-15558.

Keune, W.J., Jones, D.R., Bultsma, Y., Sommer, L., Zhou, X.Z., Lu, K.P., and Divecha, N. (2012). Regulation of phosphatidylinositol-5-phosphate signaling by Pin1 determines sensitivity to oxidative stress. Science signaling 5, ra86.

Kim, J.K., Esteve, P.O., Jacobsen, S.E., and Pradhan, S. (2009). UHRF1 binds G9a and participates in p21 transcriptional regulation in mammalian cells. Nucleic Acids Res 37, 493-505.

Kouzarides, T. (2007). Chromatin modifications and their function. Cell 128, 693-705.

Kunkel, T.A. (1985). Rapid and efficient site-specific mutagenesis without phenotypic selection. Proc Natl Acad Sci U S A 82, 488-492.

Laemmli, U.K. (1970). Cleavage of structural proteins during the assembly of the head of bacteriophage T4. Nature 227, 680-685.

Lallous, N., Legrand, P., McEwen, A.G., Ramon-Maiques, S., Samama, J.P., and Birck, C. (2011). The PHD finger of human UHRF1 reveals a new subgroup of unmethylated histone H3 tail readers. PLoS One 6, e27599.

Latour, S., Zhang, J., Siraganian, R.P., and Veillette, A. (1998). A unique insert in the linker domain of Syk is necessary for its function in immunoreceptor signalling. The EMBO journal 17, 2584-2595.

Lehnertz, B., Ueda, Y., Derijck, A.A., Braunschweig, U., Perez-Burgos, L., Kubicek, S., Chen, T., Li, E., Jenuwein, T., and Peters, A.H. (2003). Suv39h-mediated histone H3 lysine 9 methylation directs DNA methylation to major satellite repeats at pericentric heterochromatin. Curr Biol 13, 1192-1200. 
Lewis, A.E., Sommer, L., Arntzen, M.O., Strahm, Y., Morrice, N.A., Divecha, N., and D'Santos, C.S. (2011). Identification of nuclear phosphatidylinositol 4,5-bisphosphateinteracting proteins by neomycin extraction. Mol Cell Proteomics 10, M110 003376.

Li, W., Dou, S.X., Xie, P., and Wang, P.Y. (2007). Brownian dynamics simulation of the effect of histone modification on nucleosome structure. Phys Rev E Stat Nonlin Soft Matter Phys 75, 051915.

Liang, C.C., Zhan, B., Yoshikawa, Y., Haas, W., Gygi, S.P., and Cohn, M.A. (2015). UHRF1 is a sensor for DNA interstrand crosslinks and recruits FANCD2 to initiate the Fanconi anemia pathway. Cell Rep 10, 1947-1956.

Lister, R., Pelizzola, M., Dowen, R.H., Hawkins, R.D., Hon, G., Tonti-Filippini, J., Nery, J.R., Lee, L., Ye, Z., Ngo, Q.M., et al. (2009). Human DNA methylomes at base resolution show widespread epigenomic differences. Nature 462, 315-322.

Liu, X., Gao, Q., Li, P., Zhao, Q., Zhang, J., Li, J., Koseki, H., and Wong, J. (2013). UHRF1 targets DNMT1 for DNA methylation through cooperative binding of hemi-methylated DNA and methylated H3K9. Nat Commun 4, 1563.

Lu, C., and Thompson, C.B. (2012). Metabolic regulation of epigenetics. Cell Metab 16, 917.

Luger, K., Dechassa, M.L., and Tremethick, D.J. (2012). New insights into nucleosome and chromatin structure: an ordered state or a disordered affair? Nature reviews Molecular cell biology 13, 436-447.

Luger, K., Mader, A.W., Richmond, R.K., Sargent, D.F., and Richmond, T.J. (1997). Crystal structure of the nucleosome core particle at 2.8 A resolution. Nature 389, 251-260.

Lundby, A., Secher, A., Lage, K., Nordsborg, N.B., Dmytriyev, A., Lundby, C., and Olsen, J.V. (2012). Quantitative maps of protein phosphorylation sites across 14 different rat organs and tissues. Nat Commun 3, 876.

Ma, B., Tsai, C.J., Haliloglu, T., and Nussinov, R. (2011). Dynamic allostery: linkers are not merely flexible. Structure 19, 907-917.

Ma, H., Chen, H., Guo, X., Wang, Z., Sowa, M.E., Zheng, L., Hu, S., Zeng, P., Guo, R., Diao, J., et al. (2012). M phase phosphorylation of the epigenetic regulator UHRF1 regulates its physical association with the deubiquitylase USP7 and stability. Proc Natl Acad Sci U S A 109, 4828-4833.

Maison, C., and Almouzni, G. (2004). HP1 and the dynamics of heterochromatin maintenance. Nature reviews Molecular cell biology 5, 296-304. 
Manzoli, F.A., Cocco, L., Facchini, A., Casali, A.M., Maraldi, N.M., and Grossi, C.E. (1976). Phospholipids bound to acidic nuclear proteins in human B and T lymphocytes. Mol Cell Biochem 12, 67-71.

Matsuda, Y., and Chapman, V.M. (1991). In situ analysis of centromeric satellite DNA segregating in Mus species crosses. Mamm Genome 1, 71-77.

McGhee, J.D., and Felsenfeld, G. (1980). Nucleosome structure. Annu Rev Biochem 49, 1115-1156.

McGinty, R.K., and Tan, S. (2015). Nucleosome structure and function. Chem Rev 115, 2255-2273.

Meilinger, D., Fellinger, K., Bultmann, S., Rothbauer, U., Bonapace, I.M., Klinkert, W.E., Spada, F., and Leonhardt, H. (2009). Np95 interacts with de novo DNA methyltransferases, Dnmt3a and Dnmt3b, and mediates epigenetic silencing of the viral CMV promoter in embryonic stem cells. EMBO Rep 10, 1259-1264.

Meshkini, A., and Yazdanparast, R. (2010). Chemosensitization of human leukemia K562 cells to taxol by a Vanadium-salen complex. Exp Mol Pathol 89, 334-342.

Millar, C.B., and Grunstein, M. (2006). Genome-wide patterns of histone modifications in yeast. Nature reviews Molecular cell biology 7, 657-666.

Mistry, H., Gibson, L., Yun, J.W., Sarras, H., Tamblyn, L., and McPherson, J.P. (2008). Interplay between Np95 and Eme1 in the DNA damage response. Biochem Biophys Res Commun 375, 321-325.

Mistry, H., Tamblyn, L., Butt, H., Sisgoreo, D., Gracias, A., Larin, M., Gopalakrishnan, K., Hande, M.P., and McPherson, J.P. (2010). UHRF1 is a genome caretaker that facilitates the DNA damage response to gamma-irradiation. Genome Integr 1, 7.

Miura, M., Watanabe, H., Sasaki, T., Tatsumi, K., and Muto, M. (2001). Dynamic changes in subnuclear NP95 location during the cell cycle and its spatial relationship with DNA replication foci. Exp Cell Res 263, 202-208.

Mousli, M., Hopfner, R., Abbady, A.Q., Monte, D., Jeanblanc, M., Oudet, P., Louis, B., and Bronner, C. (2003). ICBP90 belongs to a new family of proteins with an expression that is deregulated in cancer cells. Br J Cancer 89, 120-127.

Mullis, K., Faloona, F., Scharf, S., Saiki, R., Horn, G., and Erlich, H. (1986). Specific enzymatic amplification of DNA in vitro: the polymerase chain reaction. Cold Spring Harb Symp Quant Biol 51 Pt 1, 263-273.

Myrianthopoulos, V., Cartron, P.F., Klimašauskas, S., Matulis, D., Bronner, C., Martinet, N., and Mikros, E. (2016). Tandem virtual screening targeting the SRA domain of UHRF1 
identifies a novel chemical tool modulating DNA methylation. European Journal of Medicinal Chemistry.

Nady, N., Lemak, A., Walker, J.R., Avvakumov, G.V., Kareta, M.S., Achour, M., Xue, S., Duan, S., Allali-Hassani, A., Zuo, X., et al. (2011). Recognition of multivalent histone states associated with heterochromatin by UHRF1 protein. J Biol Chem 286, 24300-24311.

Nanney, D.L. (1958). Epigenetic control systems. Proceedings of the National Academy of Sciences 44, 712-717.

Nathan, D., Ingvarsdottir, K., Sterner, D.E., Bylebyl, G.R., Dokmanovic, M., Dorsey, J.A., Whelan, K.A., Krsmanovic, M., Lane, W.S., Meluh, P.B., et al. (2006). Histone sumoylation is a negative regulator in Saccharomyces cerevisiae and shows dynamic interplay with positive-acting histone modifications. Genes Dev 20, 966-976.

Ndamukong, I., Jones, D.R., Lapko, H., Divecha, N., and Avramova, Z. (2010). Phosphatidylinositol 5-phosphate links dehydration stress to the activity of ARABIDOPSIS TRITHORAX-LIKE factor ATX1. PLoS One 5, e13396.

Nelson, C.J., Santos-Rosa, H., and Kouzarides, T. (2006). Proline isomerization of histone H3 regulates lysine methylation and gene expression. Cell 126, 905-916.

Nishiyama, A., Yamaguchi, L., Sharif, J., Johmura, Y., Kawamura, T., Nakanishi, K., Shimamura, S., Arita, K., Kodama, T., Ishikawa, F., et al. (2013). Uhrf1-dependent H3K23 ubiquitylation couples maintenance DNA methylation and replication. Nature 502, 249253.

Nowak, S.J., and Corces, V.G. (2004). Phosphorylation of histone H3: a balancing act between chromosome condensation and transcriptional activation. Trends Genet 20, 214220.

O'Loghlen, A., Munoz-Cabello, A.M., Gaspar-Maia, A., Wu, H.A., Banito, A., Kunowska, N., Racek, T., Pemberton, H.N., Beolchi, P., Lavial, F., et al. (2012). MicroRNA regulation of Cbx7 mediates a switch of Polycomb orthologs during ESC differentiation. Cell Stem Cell 10, 33-46.

Obata, Y., Furusawa, Y., Endo, T.A., Sharif, J., Takahashi, D., Atarashi, K., Nakayama, M., Onawa, S., Fujimura, Y., Takahashi, M., et al. (2014). The epigenetic regulator Uhrf1 facilitates the proliferation and maturation of colonic regulatory T cells. Nat Immunol 15, 571-579.

Olsen, J.V., Blagoev, B., Gnad, F., Macek, B., Kumar, C., Mortensen, P., and Mann, M. (2006). Global, in vivo, and site-specific phosphorylation dynamics in signaling networks. Cell 127, 635-648. 
Osborn, L., Kunkel, S., and Nabel, G.J. (1989). Tumor necrosis factor alpha and interleukin 1 stimulate the human immunodeficiency virus enhancer by activation of the nuclear factor kappa B. Proc Natl Acad Sci U S A 86, 2336-2340.

Papait, R., Pistore, C., Grazini, U., Babbio, F., Cogliati, S., Pecoraro, D., Brino, L., Morand, A.L., Dechampesme, A.M., Spada, F., et al. (2008). The PHD domain of Np95 (mUHRF1) is involved in large-scale reorganization of pericentromeric heterochromatin. Molecular biology of the cell 19, 3554-3563.

Papait, R., Pistore, C., Negri, D., Pecoraro, D., Cantarini, L., and Bonapace, I.M. (2007). Np95 is implicated in pericentromeric heterochromatin replication and in major satellite silencing. Molecular biology of the cell 18, 1098-1106.

Paris, L.L., Hu, J., Galan, J., Ong, S.S., Martin, V.A., Ma, H., Tao, W.A., Harrison, M.L., and Geahlen, R.L. (2010). Regulation of Syk by phosphorylation on serine in the linker insert. J Biol Chem 285, 39844-39854.

Parrilla, I., Vazquez, J.M., Cuello, C., Gil, M.A., Roca, J., Di Berardino, D., and Martinez, E.A. (2004). Hoechst 33342 stain and u.v. laser exposure do not induce genotoxic effects in flow-sorted boar spermatozoa. Reproduction 128, 615-621.

Pawson, T., Gish, G.D., and Nash, P. (2001). SH2 domains, interaction modules and cellular wiring. Trends Cell Biol 11, 504-511.

Pendaries, C., Tronchere, H., Racaud-Sultan, C., Gaits-Iacovoni, F., Coronas, S., Manenti, S., Gratacap, M.P., Plantavid, M., and Payrastre, B. (2005). Emerging roles of phosphatidylinositol monophosphates in cellular signaling and trafficking. Adv Enzyme Regul 45, 201-214.

Pichler, G., Wolf, P., Schmidt, C.S., Meilinger, D., Schneider, K., Frauer, C., Fellinger, K., Rottach, A., and Leonhardt, H. (2011). Cooperative DNA and histone binding by Uhrf2 links the two major repressive epigenetic pathways. J Cell Biochem 112, 2585-2593.

Polach, K.J., Lowary, P.T., and Widom, J. (2000). Effects of core histone tail domains on the equilibrium constants for dynamic DNA site accessibility in nucleosomes. J Mol Biol 298, 211-223.

Proudhon, C., Hao, B., Raviram, R., Chaumeil, J., and Skok, J.A. (2015). Long-Range Regulation of V(D)J Recombination. Adv Immunol 128, 123-182.

Qin, W., Wolf, P., Liu, N., Link, S., Smets, M., La Mastra, F., Forne, I., Pichler, G., Horl, D., Fellinger, K., et al. (2015). DNA methylation requires a DNMT1 ubiquitin interacting motif (UIM) and histone ubiquitination. Cell Res 25, 911-929. 
Raddatz, G., Guzzardo, P.M., Olova, N., Fantappie, M.R., Rampp, M., Schaefer, M., Reik, W., Hannon, G.J., and Lyko, F. (2013). Dnmt2-dependent methylomes lack defined DNA methylation patterns. Proc Natl Acad Sci U S A 110, 8627-8631.

Rajakumara, E., Wang, Z., Ma, H., Hu, L., Chen, H., Lin, Y., Guo, R., Wu, F., Li, H., Lan, F., et al. (2011). PHD finger recognition of unmodified histone H3R2 links UHRF1 to regulation of euchromatic gene expression. Mol Cell 43, 275-284.

Richards, E.J., and Elgin, S.C. (2002). Epigenetic codes for heterochromatin formation and silencing: rounding up the usual suspects. Cell 108, 489-500.

Rigbolt, K.T., Prokhorova, T.A., Akimov, V., Henningsen, J., Johansen, P.T., Kratchmarova, I., Kassem, M., Mann, M., Olsen, J.V., and Blagoev, B. (2011). System-wide temporal characterization of the proteome and phosphoproteome of human embryonic stem cell differentiation. Sci Signal 4, rs3.

Riggs, A.D. (1975). X inactivation, differentiation, and DNA methylation. Cytogenet Cell Genet 14, 9-25.

Rose, H.G., and Frenster, J.H. (1965). Composition and Metabolism of Lipids within Repressed and Active Chromatin of Interphase Lymphocytes. Biochimica Et Biophysica Acta 106, 577-\&.

Rostam, M.A., Piva, T.J., Rezaei, H.B., Kamato, D., Little, P.J., Zheng, W., and Osman, N. (2015). Peptidyl-prolyl isomerases: Functionality and potential therapeutic targets in cardiovascular disease. Clinical and Experimental Pharmacology and Physiology 42, 117124.

Rothbart, S.B., Dickson, B.M., Ong, M.S., Krajewski, K., Houliston, S., Kireev, D.B., Arrowsmith, C.H., and Strahl, B.D. (2013). Multivalent histone engagement by the linked tandem Tudor and PHD domains of UHRF1 is required for the epigenetic inheritance of DNA methylation. Genes Dev 27, 1288-1298.

Rothbart, S.B., Krajewski, K., Nady, N., Tempel, W., Xue, S., Badeaux, A.I., BarsyteLovejoy, D., Martinez, J.Y., Bedford, M.T., Fuchs, S.M., et al. (2012). Association of UHRF1 with methylated H3K9 directs the maintenance of DNA methylation. Nat Struct Mol Biol $19,1155-1160$.

Rottach, A., Frauer, C., Pichler, G., Bonapace, I.M., Spada, F., and Leonhardt, H. (2010). The multi-domain protein Np95 connects DNA methylation and histone modification. Nucleic Acids Res 38, 1796-1804.

Sabatino, L., Fucci, A., Pancione, M., Carafa, V., Nebbioso, A., Pistore, C., Babbio, F., Votino, C., Laudanna, C., Ceccarelli, M., et al. (2012). UHRF1 coordinates peroxisome 
proliferator activated receptor gamma (PPARG) epigenetic silencing and mediates colorectal cancer progression. Oncogene 31, 5061-5072.

Saksouk, N., Barth, T.K., Ziegler-Birling, C., Olova, N., Nowak, A., Rey, E., MateosLangerak, J., Urbach, S., Reik, W., Torres-Padilla, M.E., et al. (2014). Redundant mechanisms to form silent chromatin at pericentromeric regions rely on BEND3 and DNA methylation. Mol Cell 56, 580-594.

Saksouk, N., Simboeck, E., and Dejardin, J. (2015). Constitutive heterochromatin formation and transcription in mammals. Epigenetics Chromatin 8, 3 .

Sambrook, J., and Russell, D. (2001). Molecular Cloning: A Laboratory Manual . 2001 (Cold Spring Harbor Laboratory Press, Cold Spring Harbor, New York).

Sarkar, P., Saleh, T., Tzeng, S.R., Birge, R.B., and Kalodimos, C.G. (2011). Structural basis for regulation of the Crk signaling protein by a proline switch. Nat Chem Biol 7, 51-57.

Sharif, J., and Koseki, H. (2011). Recruitment of Dnmt1 roles of the SRA protein Np95 (Uhrf1) and other factors. Progress in molecular biology and translational science 101, 289310.

Sharif, J., Muto, M., Takebayashi, S., Suetake, I., Iwamatsu, A., Endo, T.A., Shinga, J., Mizutani-Koseki, Y., Toyoda, T., Okamura, K., et al. (2007). The SRA protein Np95 mediates epigenetic inheritance by recruiting Dnmt1 to methylated DNA. Nature 450, 908-912.

Shi, X., Hong, T., Walter, K.L., Ewalt, M., Michishita, E., Hung, T., Carney, D., Pena, P., Lan, F., Kaadige, M.R., et al. (2006). ING2 PHD domain links histone H3 lysine 4 methylation to active gene repression. Nature 442, 96-99.

Shilatifard, A. (2006). Chromatin modifications by methylation and ubiquitination: implications in the regulation of gene expression. Annu Rev Biochem 75, 243-269.

Shisheva, A. (2013). PtdIns5P: news and views of its appearance, disappearance and deeds. Arch Biochem Biophys 538, 171-180.

Staynov, D.Z. (2008). The controversial $30 \mathrm{~nm}$ chromatin fibre. Bioessays 30, 1003-1009.

Sterner, D.E., and Berger, S.L. (2000). Acetylation of histones and transcription-related factors. Microbiol Mol Biol Rev 64, 435-459.

Strahl, B.D., and Allis, C.D. (2000). The language of covalent histone modifications. Nature 403, 41-45.

Struhl, K. (1999). Fundamentally different logic of gene regulation in eukaryotes and prokaryotes. Cell 98, 1-4. 
Su, R.-C., Brown, K.E., Saaber, S., Fisher, A.G., Merkenschlager, M., and Smale, S.T. (2004). Dynamic assembly of silent chromatin during thymocyte maturation. Nature genetics 36 , 502-506.

Takeuchi, K., Sun, Z.Y., Park, S., and Wagner, G. (2010). Autoinhibitory interaction in the multidomain adaptor protein Nck: possible roles in improving specificity and functional diversity. Biochemistry 49, 5634-5641.

Tauber, M., and Fischle, W. (2015). Conserved linker regions and their regulation determine multiple chromatin-binding modes of UHRF1. Nucleus 6, 123-132.

Tian, Y., Paramasivam, M., Ghosal, G., Chen, D., Shen, X., Huang, Y., Akhter, S., Legerski, R., Chen, J., Seidman, M.M., et al. (2015). UHRF1 contributes to DNA damage repair as a lesion recognition factor and nuclease scaffold. Cell Rep 10, 1957-1966.

Tien, A.L., Senbanerjee, S., Kulkarni, A., Mudbhary, R., Goudreau, B., Ganesan, S., Sadler, K.C., and Ukomadu, C. (2011). UHRF1 depletion causes a G2/M arrest, activation of DNA damage response and apoptosis. Biochem J 435, 175-185.

Tota, M.R., Daniel, S., Sirotina, A., Mazina, K.E., Fong, T.M., Longmore, J., and Strader, C.D. (1994). Characterization of a fluorescent substance P analog. Biochemistry 33, 1307913086.

Towbin, H., Staehelin, T., and Gordon, J. (1979). Electrophoretic transfer of proteins from polyacrylamide gels to nitrocellulose sheets: procedure and some applications. Proc Natl Acad Sci U S A 76, 4350-4354.

Trojer, P., and Reinberg, D. (2007). Facultative heterochromatin: is there a distinctive molecular signature? Mol Cell 28, 1-13.

Tsumura, A., Hayakawa, T., Kumaki, Y., Takebayashi, S., Sakaue, M., Matsuoka, C., Shimotohno, K., Ishikawa, F., Li, E., Ueda, H.R., et al. (2006). Maintenance of self-renewal ability of mouse embryonic stem cells in the absence of DNA methyltransferases Dnmt1, Dnmt3a and Dnmt3b. Genes Cells 11, 805-814.

Unoki, M., Brunet, J., and Mousli, M. (2009). Drug discovery targeting epigenetic codes: the great potential of UHRF1, which links DNA methylation and histone modifications, as a drug target in cancers and toxoplasmosis. Biochem Pharmacol 78, 1279-1288.

Unoki, M., Daigo, Y., Koinuma, J., Tsuchiya, E., Hamamoto, R., and Nakamura, Y. (2010). UHRF1 is a novel diagnostic marker of lung cancer. Br J Cancer 103, 217-222.

Unoki, M., Nishidate, T., and Nakamura, Y. (2004). ICBP90, an E2F-1 target, recruits HDAC1 and binds to methyl-CpG through its SRA domain. Oncogene 23, 7601-7610. 
Vesterberg, O., Hansen, L., and Sjosten, A. (1977). Staining of proteins after isoelectric focusing in gels by new procedures. Biochim Biophys Acta 491, 160-166.

Waddington, C.H. (2012). The epigenotype. 1942. Int J Epidemiol 41, 10-13.

Wang, F., Yang, Y.Z., Shi, C.Z., Zhang, P., Moyer, M.P., Zhang, H.Z., Zou, Y., and Qin, H.L. (2012). UHRF1 promotes cell growth and metastasis through repression of p16(ink(4)a) in colorectal cancer. Ann Surg Oncol 19, 2753-2762.

Wang, X., and Hayes, J.J. (2007). Site-specific binding affinities within the H2B tail domain indicate specific effects of lysine acetylation. J Biol Chem 282, 32867-32876.

Wang, Z., Song, J., Milne, T.A., Wang, G.G., Li, H., Allis, C.D., and Patel, D.J. (2010). Pro isomerization in MLL1 PHD3-bromo cassette connects H3K4me readout to CyP33 and HDAC-mediated repression. Cell 141, 1183-1194.

Whitfield, M.L., Zheng, L.X., Baldwin, A., Ohta, T., Hurt, M.M., and Marzluff, W.F. (2000). Stem-loop binding protein, the protein that binds the $3^{\prime}$ end of histone mRNA, is cell cycle regulated by both translational and posttranslational mechanisms. Mol Cell Biol 20, 41884198.

Xie, S., Jakoncic, J., and Qian, C. (2012). UHRF1 double tudor domain and the adjacent PHD finger act together to recognize K9me3-containing histone H3 tail. J Mol Biol 415, 318-328.

Yao, J., Leng, L., Sauler, M., Fu, W., Zheng, J., Zhang, Y., Du, X., Yu, X., Lee, P., and Bucala, R. (2016). Transcription factor ICBP90 regulates the MIF promoter and immune susceptibility locus. J Clin Invest 126, 732-744.

Zemach, A., McDaniel, I.E., Silva, P., and Zilberman, D. (2010). Genome-wide evolutionary analysis of eukaryotic DNA methylation. Science 328, 916-919.

Zhang, Y., and Reinberg, D. (2001). Transcription regulation by histone methylation: interplay between different covalent modifications of the core histone tails. Genes Dev 15, 2343-2360.

Zhang, Z.M., Rothbart, S.B., Allison, D.F., Cai, Q., Harrison, J.S., Li, L., Wang, Y., Strahl, B.D., Wang, G.G., and Song, J. (2015). An Allosteric Interaction Links USP7 to Deubiquitination and Chromatin Targeting of UHRF1. Cell Rep 12, 1400-1406.

Zhao, K., Wang, W., Rando, O.J., Xue, Y., Swiderek, K., Kuo, A., and Crabtree, G.R. (1998). Rapid and phosphoinositol-dependent binding of the SWI/SNF-like BAF complex to chromatin after T lymphocyte receptor signaling. Cell 95, 625-636.

Zheng, C., and Hayes, J.J. (2003). Structures and interactions of the core histone tail domains. Biopolymers 68, 539-546. 


\section{$7 \quad$ Addendum}

\section{1 cDNA sequences}

The coding sequence of homo sapiens UHRF1/ICBP90 was used as published in (Gelato et al., 2014)

hUHRF1 (ICBP90, 2379 bp)

ATGTGGATCCAGGTTCGGACCATGGACGGGAGGCAGACCCACACGGTGGACTCGCT GTCCAGGCTGACCAAGGTGGAGGAGCTGAGGCGGAAGATCCAGGAGCTGTTCCACG TGGAGCCAGGCCTGCAGAGGCTGTTCTACAGGGGCAAACAGATGGAGGACGGCCAT ACCCTCTTCGACTACGAGGTCCGCCTGAATGACACCATCCAGCTCCTGGTCCGCCAG AGCCTCGTGCTCCCCCACAGCACCAAGGAGCGGGACTCCGAGCTCTCCGACACCGA CTCCGGCTGCTGCCTGGGCCAGAGTGAGTCAGACAAGTCCTCCACCCACGGTGAGG CGGCCGCCGAGACTGACAGCAGGCCAGCCGATGAGGACATGTGGGATGAGACGGAA TTGGGGCTGTACAAGGTCAATGAGTACGTCGATGCTCGGGACACGAACATGGGGGC GTGGTTTGAGGCGCAGGTGGTCAGGGTGACGCGGAAGGCCCCCTCCCGGGACGAG CCCTGCAGCTCCACGTCCAGGCCGGCGCTGGAGGAGGACGTCATTTACCACGTGAA ATACGACGACTACCCGGAGAACGGCGTGGTCCAGATGAACTCCAGGGACGTCCGAG CGCGCGCCCGCACCATCATCAAGTGGCAGGACCTGGAGGTGGGCCAGGTGGTCATG CTCAACTACAACCCCGACAACCCCAAGGAGCGGGGCTTCTGGTACGACGCGGAGATC TCCAGGAAGCGCGAGACCAGGACGGCGCGGGAACTCTACGCCAACGTGGTGCTGGG GGATGATTCTCTGAACGACTGTCGGATCATCTTCGTGGACGAAGTCTTCAAGATTGAG CGGCCGGGTGAAGGGAGCCCCATGGTTGACAACCCCATGAGACGGAAGAGCGGGCC GTCCTGCAAGCACTGCAAGGACGACGTGAACAGACTCTGCCGGGTCTGCGCCTGCC ACCTGTGCGGGGGCCGGCAGGACCCCGACAAGCAGCTCATGTGCGATGAGTGCGAC ATGGCCTTCCACATCTACTGCCTGGACCCGCCCCTCAGCAGTGTTCCCAGCGAGGAC GAGTGGTACTGCCCTGAGTGCCGGAATGATGCCAGCGAGGTGGTACTGGCGGGAGA GCGGCTGAGAGAGAGCAAGAAGAAGGCGAAGATGGCCTCGGCCACATCGTCCTCAC AGCGGGACTGGGGCAAGGGCATGGCCTGTGTGGGCCGCACCAAGGAATGTACCATC GTCCCGTCCAACCACTACGGACCCATCCCGGGGATCCCCGTGGGCACCATGTGGCG GTTCCGAGTCCAGGTCAGCGAGTCGGGTGTCCATCGGCCCCACGTGGCTGGCATAC ACGGCCGGAGCAACGACGGAGCGTACTCCCTAGTCCTGGCGGGGGGCTATGAGGAT GATGTGGACCATGGGAATTTTTTCACATACACGGGTAGTGGTGGTCGAGATCTTTCCG GCAACAAGAGGACCGCGGAACAGTCTTGTGATCAGAAACTCACCAACACCAACAGGG CGCTGGCTCTCAACTGCTTTGCTCCCATCAATGACCAAGAAGGGGCCGAGGCCAAGG ACTGGCGGTCGGGGAAGCCGGTCAGGGTGGTGCGCAATGTCAAGGGTGGCAAGAAT AGCAAGTACGCCCCCGCTGAGGGCAACCGCTACGATGGCATCTACAAGGTTGTGAAA TACTGGCCCGAGAAGGGGAAGTCCGGGTTTCTCGTGTGGCGCTACCTTCTGCGGAG GGACGATGATGAGCCTGGCCCTTGGACGAAGGAGGGGAAGGACCGGATCAAGAAGC TGGGGCTGACCATGCAGTATCCAGAAGGCTACCTGGAAGCCCTGGCCAACCGAGAG CGAGAGAAGGAGAACAGCAAGAGGGAGGAGGAGGAGCAGCAGGAGGGGGGCTTCG CGTCCCCCAGGACGGGCAAGGGCAAGTGGAAGCGGAAGTCGGCAGGAGGTGGCCC GAGCAGGGGGTCCCCGCGCCGGACATCCAAGAAAACCAAGGTGGAGCCCTACAGTC TCACGGCCCAGCAGAGCAGCCTCATCAGAGAGGACAAGAGCAACGCCAAGCTGTGG AATGAGGTCCTGGCGTCACTCAAGGACCGGCCGGCGAGCGGCAGCCCGTTCCAGTT GTTCCTGAGTAAAGTGGAGGAGACGTTCCAGTGTATCTGCTGTCAGGAGCTGGTGTT CCGGCCCATCACGACCGTGTGCCAGCACAACGTGTGCAAGGACTGCCTGGACAGAT CCTTTCGGGCACAGGTGTTCAGCTGCCCTGCCTGCCGCTACGACCTGGGCCGCAGC TATGCCATGCAGGTGAACCAGCCTCTGCAGACCGTCCTCAACCAGCTCTTCCCCGGC TACGGCAATGGCCGGTGA 
The coding sequence of mus musculus NP95 variant I matching the annotated sequence BC022167 was obtained from Source bioscience as I.M.A.G.E. fully sequenced cDNA clone IRAVp968C1122D (ImageID: 4017286)

mNP95 variant I (NP95 I, $2349 \mathrm{bp})$

ATGTGGATCCAGGTTCGAACTATGGATGGGAAGGAGACCCACACCGTGAACTCTCTG TCCAGGTTGACCAAGGTGCAGGAGCTGAGGAAAAAGATTGAGGAAGTGTTTCACGTG GAACCCCAACTGCAGAGACTCTTTTACAGGGGCAAACAGATGGAGGACGGCCACACA CTCTTCGATTATGATGTGCGCCTCAATGACACAATCCAGCTGCTCGTGCGCCAGAGTC TGGCACTGCCTCTCAGTACAAAAGAACGGGACTCGGAGCTCTCAGACTCGGACTCTG GCTATGGTGTGGGTCACAGTGAATCAGACAAGTCGTCCACGCATGGTGAAGGGGCAG CTGAAGCGGATGACAAGACTGTGTGGGAGGACACGGACCTGGGGCTGTACAAGGTC AATGAGTATGTGGACGTGCGTGACAATATCTTCGGTGCATGGTTTGAGGCCCAGGTG GTCCAGGTACAGAAGAGAGCCCTATCTGAGGACGAGCCCTGTAGCTCCAGTGCCGTT AAGACCTCGGAGGATGACATCATGTACCATGTCAAGTATGATGACTATCCAGAGCATG GAGTGGACATTGTCAAAGCCAAGAATGTCCGTGCTCGTGCTCGCACTGTGATACCAT GGGAGAACCTGGAGGTGGGTCAGGTGGTCATGGCCAACTATAACGTGGACTACCCC AGGAAACGCGGCTTCTGGTATGATGTTGAGATCTGTAGGAAGCGCCAAACCAGGACG GCACGTGAGCTATACGGCAACATCAGGCTCTTGAATGACTCTCAGCTCAACAACTGTC GGATCATGTTTGTGGATGAAGTCTTGATGATTGAGCTCCCTAAAGAGAGGAGGCCTTT GATCGCTAGCCCCTCGCAACCCCCTCCTGCCCTCCGGAACACAGGGAAGAGCGGCC CATCCTGCCGGTTCTGCAAGGATGATGAAAACAAACCATGTCGAAAGTGTGCCTGCC ACGTGTGTGGTGGGCGCGAGGCTCCTGAGAAACAGCTGTTGTGTGATGAGTGTGACA TGGCCTTCCACCTGTACTGCCTGAAGCCACCGCTCACCTCTGTCCCCCCTGAGCCGG AGTGGTACTGCCCCAGCTGCCGCACTGACTCAAGTGAGGTGGTACAAGCAGGGGAG AAGCTGAAGGAGAGCAAGAAGAAGGCGAAGATGGCATCAGCCACTTCCTCCTCCCGG CGGGACTGGGGCAAGGGCATGGCATGTGTGGGCCGCACCACAGAGTGTACCATTGT GCCCGCCAACCACTTCGGGCCCATCCCTGGTGTCCCTGTGGGCACCATGTGGCGCTT CAGAGTCCAGGTCAGTGAGTCCGGTGTGCATCGGCCTCATGTGGCAGGCATCCATGG CCGGAGCAACGACGGTGCCTACTCATTGGTCCTGGCTGGTGGCTATGAGGATGATGT GGACAATGGCAATTACTTCACATACACAGGGAGTGGTGGCCGAGACCTCTCTGGCAA CAAGCGTACAGCAGGCCAGTCCTCTGACCAGAAGCTCACTAATAACAATAGGGCTCT GGCACTCAATTGCCACTCCCCAATCAATGAGAAAGGTGCGGAGGCTGAAGACTGGCG CCAAGGGAAGCCAGTGCGTGTGGTCCGGAACATGAAGGGCGGGAAACACAGCAAGT ACGCTCCTGCAGAGGGCAACCGCTATGATGGCATCTACAAGGTGGTGAAGTACTGGC CAGAGAGAGGGAAATCTGGCTTCCTCGTGTGGCGTTATCTCCTTCGACGAGATGACA CAGAGCCTGAGCCCTGGACCCGGGAGGGCAAGGACCGCACTCGACAGCTGGGGCT CACTATGCAGTACCCTGAAGGCTACTTGGAGGCCTTGGCTAACAAGGAGAAGAGCAG GAAGCGCCCGGCCAAGGCCTTGGAGCAGGGACCCTCATCTTCCAAGACAGGCAAAA GCAAGCAGAAGTCCACAGGGCCGACCCTCTCCAGCCCCCGTGCCTCTAAGAAGAGC AAGCTGGAGCCGTACACACTCTCAGAGCAGCAGGCTAACCTCATCAAAGAGGACAAG GGCAACGCCAAGCTGTGGGATGATGTGCTAACTTCACTTCAGGATGGGCCGTACCAG ATCTTCCTGAGCAAGGTGAAAGAGGCTTTCCAGTGCATCTGCTGCCAGGAGCTGGTG TTCCGGCCAGTCACCACCGTGTGTCAGCACAACGTCTGTAAGGACTGTCTGGACAGG TCCTTCCGGGCCCAGGTGTTCAGCTGCCCCGCCTGTCGCTATGAGCTGGACCACAGC TCCCCAACCCGGGTGAACCAGCCCTTGCAGACCATTCTCAACCAGCTCTTCCCTGGC TATGGCAGCGGCCGGTGA 
The coding sequence of mus musculus NP95 variant II matching the annotated sequence AK153083 was obtained from Source bioscience as RIKEN Mouse FANTOM full length cDNA clone I830121L22

mNP95 variant II (NP95 II, 2325 bp)

ATGTGGATCCAGGTTCGAACTATGGATGGGAAGGAGACCCACACCGTGAACTCTCTG TCCAGGTTGACCAAGGTGCAGGAGCTGAGGAAAAAGATTGAGGAAGTGTTTCACGTG GAACCCCAACTACAGAGACTCTTTTATAGGGGCAAACAGATGGAGGATGGCCACACA CTCTTCGATTATGATGTGCGCCTCAATGACACAATCCAGCTGCTCGTGCGCCAGAGTC TGGCACTGCCTCTCAGTACAAAAGAACGGGACTCGGAGCTCTCAGACTCTGACTCTG GCTATGGTGTGGGTCACAGTGAATCAGACAAGTCGTCCACGCATGGTGAAGGGGCAG CTGAAGCGGGTGACAAGACTGTGTGGGAGGACACGGACCTGGGGCTGTACAAGGTC AATGAGTATGTGGACGTGCGTGACAATATCTTCGGTGCATGGTTTGAGGCCCAGGTG GTCCAGGTACAGAAGAGAGCCCTATCTGAGGACGAGCCCTGTAGCTCCAGTGCCGTT AAGACCTCGGAGGATGACATCATGTACCATGTCAAGTATGATGACTATCCAGAGCATG GAGTGGACATTGTCAAAGCCAAGAATGTCCGTGCTCGTGCTCGCACTGTGATACCAT GGGAGAACCTGGAGGTGGGTCAGGTGGTCATGGCCAACTATAACGTGGACTACCCC AGGAAACGCGGCTTCTGGTATGATGTTGAGATCTGTAGGAAGCGCCAAACCAGGACG GCACGTGAGCTATACGGCAACATCAGGCTCTTGAATGACTCTCAGCTCAACAACTGTC GGATCATGTTTGTGGATGAAGTCTTGATGATTGAGCTCCCTAAAGAGAGGAGGCCTTT GATCGCTAGCCCCTCGCAACGGAAGAGCGGCCCATCCTGCCGGTTCTGCAAGGATG ATGAAAACAAACCATGTCGAAAGTGTGCCTGCCATGTGTGTGGTGGGCGCGAGGCTC CTGAGAAACAGCTGTTGTGTGATGAGTGTGACATGGCCTTCCACCTGTACTGCCTGAA GCCACCGCTCACCTCTGTCCCCCCTGAGCCGGAGTGGTACTGCCCCAGCTGCCGCA CTGACTCAAGTGAGGTGGTACAAGCAGGGGAGAAGCTGAAGGAGAGCAAGAAGAAG GCAAAGATGGCATCAGCCACTTCCTCCTCCCGGCGGGACTGGGGCAAGGGCATGGC ATGTGTGGGCCGCACCACAGAGTGTACCATTGTGCCCGCCAACCACTTCGGGCCCAT CCCTGGTGTCCCTGTGGGCACCATGTGGCGCTTCAGAGTCCAGGTCAGTGAGTCCG GTGTGCATCGGCCTCATGTGGCAGGCATCCATGGCCGGAGCAACGACGGTGCCTAC TCATTGGTCCTGGCTGGTGGCTATGAGGATGATGTGGACAATGGCAATTACTTCACAT ACACAGGGAGTGGTGGCCGAGACCTCTCTGGCAACAAGCGTACAGCAGGCCAGTCC TCTGACCAGAAGCTCACTAATAACAATAGGGCTCTGGCACTCAATTGCCACTCCCCAA TCAATGAGAAAGGTGCGGAGGCTGAAGACTGGCGCCAAGGGAAGCCAGTGCGTGTG GTCCGGAACATGAAGGGCGGGAAACACAGCAAGTACGCTCCTGCAGAGGGCAACCG CTATGATGGCATCTACAAGGTGGTGAAGTACTGGCCAGAGAGAGGGAAATCTGGCTT CCTCGTGTGGCGTTATCTCCTTCGACGAGATGACACAGAGCCTGAGCCCTGGACCCG GGAGGGCAAGGACCGCACTCGACAGCTGGGGCTCACTATGCAGTACCCTGAAGGCT ACTTGGAGGCCTTGGCTAACAAGGAGAAGAGCAGGAAGCGCCCGGCCAAGGCCTTG GAGCAGGGACCCTCATCTTCCAAGACAGGCAAAAGCAAGCAGAAGTCCACAGGGCCA ACCCTCTCCAGCCCCCGTGCCTCTAAGAAGAGCAAGCTGGAGCCGTACACACTCTCA GAGCAGCAGGCTAACCTCATCAAAGAGGACAAGGGCAACGCCAAGCTGTGGGATGAT GTGCTAACTTCACTTCAGGATGGGCCGTACCAGGTCTTCCTGAGCAAGGTGAAAGAG GCTTTCCAGTGCATCTGCTGCCAGGAGCTGGTGTTCCGGCCAGTCACCACCGTGTGT CAGCACAACGTCTGTAAGGACTGTCTGGACAGGTCCTTCCGGGCCCAGGTGTTCAGC TGCCCAGCCTGTCGCTTTGAGCTGGACCACAGCTCCCCAACCCGGGTGAACCAGCCC TTGCAGACCATTCTCAACCAGCTCTTCCCTGGCTATGGCAGCGGCCGGTGA 


\subsection{Primer list}

\subsubsection{Cloning Primers}

Table 7-1 Names and sequences of the used cloning primers

\begin{tabular}{|c|c|}
\hline Oligo name & Sequence \\
\hline NP95 for Kpnl pcDNA3.1 & " gatcatggatccgcatgtggatccaggttcgaac \\
\hline NP95 rev Notl pcDNA3.1 & tacatgcggccgcccggccgctgccatagccag \\
\hline pmCherry UHRF1 fwd Xhol & catcgtctcgagctatgtggatccaggttcgg \\
\hline pmCherry UHRF1 rev Xbal & catcgttctagatcaccggccattgccgtagcc \\
\hline pmCherry NP95 fwd Xhol & gatcatctcgag ct atgtggatccaggttcgaac \\
\hline pmCherry NP95 rev Xbal & gatcattctaga tcaccggccgctgccatagc \\
\hline pEGFP PBR-RING/NLS rev Kpnl & atatgcggggtaccttaccggtccttgagtgacgcc \\
\hline pEGFP PBR-RING/NLS fwd Xhol & aggatgccctcgagctgatgatgagcctggcccttg \\
\hline petM13 NP95 fwd BamHI & agctatgtggatccaggttcgaactatggatg \\
\hline petM13 NP95 rev Xhol & gtggtgctcgagccggccgctgccatagc \\
\hline pRuth NP95 V2 Kpnl fwd & agatctgggtacctggatccaggttcgaactatggatg \\
\hline pRuth NP95 V2 HindIII rev & gcggccgcaagctttcaccggccgctgccatagccag \\
\hline pcDNA3.1 NP95 fwd Kpnl & gatcatggtaccgcatgtggatccaggttcgaac \\
\hline petM13 NP95 longPHD BspHI fwd & gatactgatcatgaagagcggcccatcctg \\
\hline petM13 NP95 longPHD Xhol rev & cagaattactcgagctcccctgcttgtaccac \\
\hline petM13 NP95 TTD Xhol rev & cagaattactcgagcctcctctctttagg \\
\hline petM13 NP95 longRING BspHI fwd & gagatatatcatgattctggggctcactatg \\
\hline petM13 NP95 TTD BspHI fwd & gagatatatcatgattgtgtgggaggacacg \\
\hline
\end{tabular}

\subsubsection{Sequencing primer}

Table 7-2 Names and sequences of the used sequencing primers

\begin{tabular}{ll}
\hline Oligo name & Sequence \\
\hline \hline SRA seq Primer & ctaccttctgcggagggacgatg \\
NP95 N-term rev & tgaaacacttcctcaatctt \\
NP95 N-term fwd & aag att gag gaa gtg ttt ca \\
NP95 C-term rev & aagagctggttgagaatggt \\
NP95 C-term fwd & accattctcaaccagctctt \\
NP95 center & cactgactcaagtgaggtggta \\
\hline
\end{tabular}




\subsubsection{Mutagenesis primers}

Table 7-3 Names and sequences of the used mutagenesis primers

\begin{tabular}{|c|c|}
\hline Oligo name & Sequence \\
\hline fwd UHRF1 YT478/479AA & " ggg aat ttt ttc aca gcc gcg ggt agt gg \\
\hline rev UHRF1 YT478/479AA & tcg acc acc act acc cgc ggc tgt gaa \\
\hline fwd UHRF1 R491A & cc ggc aac aag gca acc gcg g \\
\hline rev UHRF1 R491A & ctg ttc cgc ggt tgc ctt gtt g \\
\hline UHRF1 K648A fwd & ggc aag ggc aag tgg gcg cgg aag tcg \\
\hline UHRF1 K648A rev & tcc tgc cga ctt ccg cgc cca ctt gcc \\
\hline PIP4Ka G131L/Y138F fwd & cgcagtctagctcgttttcacacttccttcgac \\
\hline PIP4Ka G131L/Y138F rev & ttgtcgaaggaagtgtgaaaacgagctagactg \\
\hline PIP4Kb D278A fwd & gatcatggcctacagcctg \\
\hline PIP4Kb D278A rev & ccagcaggctgtaggccatg \\
\hline PIPKb D278A rat fwd & gatcatggcctacagcctcctgg \\
\hline PIPKb D278A rat rev & ggctgtaggccatgatcttcagc \\
\hline PIPKa fwd G131L/Y138F & gcagcctggctcggtttcacacgtcctttgacaaaag \\
\hline PIPKa rev G131L/Y138F & tgtcaaaggacgtgtgaaaccgagccaggctgcgagc \\
\hline petM13 UHRF1 fwd His10 & ctacggcaatggccggcaccaccaccacctcgagcacc \\
\hline petM13 UHRF1 rev His10 & gtggtgctcgaggtggtggtggtgccggccattgc \\
\hline NP95 PBR-RING myc tag muta fwd & $\begin{array}{l}\text { ctggctatggcagcggccgggaacaaaaactcatctcagaagagg } \\
\text { atctgctcgagcaccaccac }\end{array}$ \\
\hline NP95 PBR-RING myc tag muta rev & $\begin{array}{l}\text { gtggtggtggtggtgctcgagcagatcctcttctgagatgagtttttgttc } \\
\text { ccggccgctgccatag }\end{array}$ \\
\hline UHRF1 TP linker Insertion NP95 I fwd & $\begin{array}{l}\text { cccatggttgacaaccccatgagaccccctcctgccctccggaacac } \\
\text { agggaagagcgggecgtcc }\end{array}$ \\
\hline UHRF1 TP linker Insertion NP95 I rev & $\begin{array}{l}\text { gcttgcaggacggcccgetcttccctgtgttccggagggcaggaggg } \\
\text { ggtctcatggggttgtc }\end{array}$ \\
\hline NP95 V1 PPP linker mut fwd & gatcgctagcccctcgcaagccgctgctgccctccggaac \\
\hline NP95 V1 PPP linker mut rev & cttccctgtgttccggagggcagcagcggcttgcgaggg \\
\hline NP95 V1 R298 linker mut fwd & gcaacccctcctgccctcgcgaacacaggg \\
\hline NP95 V1 R298 linker mut rev & gccgctcttccctgtgttcgcgagggcaggagg \\
\hline NP95 V2 RKS-AAA mutag fwd & ttgatcgctagcccctcgcaagcggcagctggcccatcctgccgg \\
\hline NP95 V2 RKS-AAA mutag rev & $\begin{array}{l}\text { ccttgcagaaccggcaggatgggccagctgccgcttgcgaggggct } \\
\text { agc }\end{array}$ \\
\hline NP95 V1 KS-AA mutag fwd & gccctccggaacacaggggcagctggcccatcctgccgg \\
\hline NP95 V1 KS-AA mutag rev & ccttgcagaaccggcaggatgggccagctgcccctgtgttccg \\
\hline NP95 TTD 3xFlag fwd & $\begin{array}{l}\text { agaaggagatataccatggactacaaggaccacgacggtgactac } \\
\text { aaggaccacgacatcgactacaaggacgacgacgacaaggtgtg } \\
\text { ggaggacacggacctggggctgtac }\end{array}$ \\
\hline NP95 TTD 3xFlag rev & $\begin{array}{l}\text { gtccgtgtcctcccacaccttgtcgtcgtcgtccttgtagtcgatgtcgtg } \\
\text { gtccttgtagtcaccgtcgtggtccttgtagtccatggtatatctccttctta }\end{array}$ \\
\hline
\end{tabular}




\begin{tabular}{|c|c|}
\hline & aagttaaac \\
\hline NP95II consensus fwd & gcagctgaagcggatgacaagactgtgtgggaggacac \\
\hline NP95II consensus rev & cccacacagtcttgtcatccgcttcagctgccccttcacc \\
\hline NP95 YY-AA TTD mt fwd & ccatgtcaaggcagatgacgcaccagagcatggagtggaca \\
\hline NP95 YY-AA TTD mt rev & gctctggtgcgtcatctgccttgacatggtacatgatgtc \\
\hline NP95 DE-AA PHD mt fwd & gctgttgtgtgctgcgtgtgacatggccttccacctgtac \\
\hline NP95 DE-AA PHD mt rev & gccatgtcacacgcagcacacaacagctgtttctcaggagc \\
\hline NP95I consens fwd & ggcctgtcgctttgagctggaccacagctcc \\
\hline NP95I consens rev & ggtccagctcaaagcgacaggccgggcagctg \\
\hline NP95 K628/632A fwd & $\begin{array}{l}\text { gagaagagcagggetcgcccggccgcagccttggagcagggacc } \\
\text { ctca }\end{array}$ \\
\hline NP95 K628/632A rev & ctgctccaaggctgcggccgggcgagccctgctcttctccttgttagc \\
\hline NP95 K649A fwd & ggcaaaagcaagcaggcatccacagggccgaccctct \\
\hline NP95 K649A rev & ggccetgtggatgcctgcttgcttttgcctgtcttgga \\
\hline NP95 K645/647/649A fwd & $\begin{array}{l}\text { cttccaagacaggcgcaagcgctcaggcatccacagggecgaccc } \\
\text { tctcc }\end{array}$ \\
\hline NP95 K645/647/649A rev & ggccctgtggatgcctgagcgcttgcgcctgtcttggaagatgaggg \\
\hline NP95 K623/K625/R627/K628/R629A fwd & $\begin{array}{l}\text { gaggccttggctaacgcagaggctagcgcagctgcgccggccaag } \\
\text { gccttggagcagggaccc }\end{array}$ \\
\hline NP95 K623/K625/R627/K628/R629A rev & $\begin{array}{l}\text { caaggccttggccggcgcagctgcgctagcctctgcgttagccaag } \\
\text { gcctccaagtagccttcagg }\end{array}$ \\
\hline
\end{tabular}

\subsection{Mass spectrometry results}

UHRF1 crosslinks with hemimethylated DNA 12mer

\begin{tabular}{crr}
\multicolumn{4}{c}{ Values } \\
Row Labels & Max of Score & Count of Spectrum \\
\hline UHRF1_HUMAN & $\mathbf{1 3 , 6 1}$ & $\mathbf{5 1}$ \\
\hline UHRF1_HUMAN & $\mathbf{1 3 , 6 1}$ & $\mathbf{5 1}$ \\
31 & 5 & 1 \\
24 & 5 & 1 \\
162 & 4,06 & 2 \\
644 & 4,06 & 2 \\
303 & 3,63 & 1 \\
650 & 3,63 & 1 \\
306 & 9,4 & 1 \\
650 & 9,4 & 1
\end{tabular}




\begin{tabular}{|c|c|c|}
\hline 385 & 7,28 & 1 \\
\hline 650 & 7,28 & 1 \\
\hline 490 & 5,66 & 4 \\
\hline 540 & 5,66 & 4 \\
\hline 500 & 5 & 1 \\
\hline 490 & 5 & 1 \\
\hline 525 & 7,89 & 2 \\
\hline 563 & 7,89 & 2 \\
\hline 540 & 4,52 & 2 \\
\hline 162 & 4,52 & 1 \\
\hline 644 & 3,26 & 1 \\
\hline 546 & 6,86 & 4 \\
\hline 24 & 6,86 & 2 \\
\hline 563 & 6,69 & 1 \\
\hline 688 & 4,39 & 1 \\
\hline 563 & 6,88 & 1 \\
\hline 24 & 6,88 & 1 \\
\hline 592 & 13,61 & 7 \\
\hline 546 & 13,61 & 7 \\
\hline 595 & 4,22 & 1 \\
\hline 644 & 4,22 & 1 \\
\hline 622 & 6,82 & 5 \\
\hline 540 & 5,88 & 4 \\
\hline 650 & 6,82 & 1 \\
\hline 626 & 6,87 & 4 \\
\hline 540 & 6,21 & 3 \\
\hline 650 & 6,87 & 1 \\
\hline 650 & 8,81 & 11 \\
\hline 297 & 5,48 & 1 \\
\hline 540 & 5,7 & 2 \\
\hline 622 & 8,66 & 3 \\
\hline 626 & 4,58 & 1 \\
\hline 644 & 4,09 & 2 \\
\hline 688 & 8,81 & 2 \\
\hline 670 & 8,56 & 2 \\
\hline 650 & 8,56 & 1 \\
\hline 702 & 4,32 & 1 \\
\hline 688 & 4,09 & 1 \\
\hline 162 & 4,09 & 1 \\
\hline otal & 13,61 & 51 \\
\hline
\end{tabular}


NP95 I crosslinks with hemimethylated DNA 12mer

\begin{tabular}{|c|c|c|}
\hline Row Labels & Max of Score & Count of Spectrum \\
\hline UHRF1_MOUSE & 14,11 & 52 \\
\hline UHRF1_MOUSE & 14,11 & 52 \\
\hline 1 & 4,37 & 1 \\
\hline 628 & 4,37 & 1 \\
\hline 32 & 5,84 & 2 \\
\hline 25 & 5,84 & 2 \\
\hline 85 & 7,01 & 1 \\
\hline 25 & 7,01 & 1 \\
\hline 197 & 10,31 & 4 \\
\hline 25 & 10,31 & 2 \\
\hline 642 & 4,73 & 1 \\
\hline 649 & 6,8 & 1 \\
\hline 199 & 4,55 & 1 \\
\hline 645 & 4,55 & 1 \\
\hline 382 & 10,22 & 1 \\
\hline 391 & 10,22 & 1 \\
\hline 384 & 3,66 & 1 \\
\hline 645 & 3,66 & 1 \\
\hline 387 & 6,81 & 2 \\
\hline 628 & 6,81 & 2 \\
\hline 391 & 8,15 & 4 \\
\hline 1 & 6,64 & 1 \\
\hline 387 & 7,31 & 2 \\
\hline 628 & 8,15 & 1 \\
\hline 506 & 5,42 & 2 \\
\hline 496 & 5,42 & 2 \\
\hline 525 & 4,71 & 1 \\
\hline 568 & 4,71 & 1 \\
\hline 536 & 5,02 & 1 \\
\hline 548 & 5,02 & 1 \\
\hline 551 & 4,9 & 3 \\
\hline 1 & 4,9 & 1 \\
\hline 25 & 4,71 & 2 \\
\hline 568 & 6,82 & 1 \\
\hline 25 & 6,82 & 1 \\
\hline 628 & 8,06 & 1 \\
\hline 199 & 8,06 & 1 \\
\hline 632 & 8,6 & 2 \\
\hline 389 & 8,6 & 1 \\
\hline 649 & 6,95 & 1 \\
\hline
\end{tabular}




\begin{tabular}{crr}
642 & 14,11 & 10 \\
1 & 10,55 & 1 \\
649 & 14,11 & 7 \\
683 & 7,57 & 2 \\
649 & 7,56 & 4 \\
568 & 4,65 & 1 \\
575 & 7,56 & 1 \\
683 & 4,79 & 2 \\
663 & 5,27 & 1 \\
632 & 5,27 & 1 \\
665 & 10,07 & 8 \\
25 & 5 & 1 \\
568 & 4,99 & 1 \\
642 & 8,97 & 2 \\
649 & 10,07 & 4 \\
683 & 4,19 & 1 \\
645 & 4,19 & 1 \\
\hline Grand Total & $\mathbf{1 4 , 1 1}$ & $\mathbf{5 2}$
\end{tabular}

NP95 I crosslinks with hemimethylated DNA 12mer

\begin{tabular}{|c|c|c|}
\hline Row Labels & Max of Score & Count of Spectrum \\
\hline UHRF1_MOUSE & 14,52 & \\
\hline UHRF1_MOUSE & 14,52 & \\
\hline 31 & 5,11 & \\
\hline 24 & 5,11 & \\
\hline 84 & 7 & \\
\hline 24 & 7 & \\
\hline 196 & 10,92 & \\
\hline 24 & 10,92 & \\
\hline 633 & 7 & \\
\hline 640 & 6 & \\
\hline 373 & 11,47 & \\
\hline 382 & 11,47 & \\
\hline 633 & 9,89 & \\
\hline 640 & 6,26 & \\
\hline 375 & 3,94 & \\
\hline 636 & 3,94 & \\
\hline
\end{tabular}


Addendum

$\begin{array}{crr}378 & 6,04 & 1 \\ 619 & 6,04 & 1 \\ 380 & 5,25 & 1 \\ 1 & 5,25 & 1 \\ 382 & 7,41 & 1 \\ 619 & 7,41 & 1 \\ 516 & 5,15 & 1 \\ 559 & 5,15 & 1 \\ 633 & 14,52 & 5 \\ 640 & 14,52 & 4 \\ 674 & 4,94 & 1 \\ 640 & 6,98 & 2 \\ 24 & 5,52 & 1 \\ 566 & 6,98 & 1 \\ 656 & 11,69 & 8 \\ 1 & 6,85 & 1 \\ 633 & 11,69 & 3 \\ 640 & 11,58 & 4 \\ \text { Grand Total } & \mathbf{1 4 , 5 2} & \mathbf{2 9}\end{array}$




\section{Curriculum vitae}

personal data:

$\begin{array}{ll}\text { Name: } & \text { Maria Tauber } \\ \text { Address: } & \text { Petrikirchstraße 42 } \\ & 37077 \text { Goettingen } \\ \text { E-Mail: } & \text { mtauber@mpibpc.mpg.de } \\ & \text { mtauber@gwdg.de }\end{array}$

Tel.:

0049-551-2011709

PhD:

since 01.02.2012 PhD student at the Max Planck Institute for biophysical chemistry, Goettingen; in chromatin biochemistry with Dr. W. Fischle

\section{Studies:}

26.09.2011 Diploma degree in biology

2005-2011 Study of biology at the Martin-Luther-University, Halle/Saale (Germany)

\section{Education:}

2005 Abitur at Christoph-Graupner-Gymnasium, Kirchberg

2000-2005 Christoph-Graupner-Gymnasium in Kirchberg (Germany)

1997-2000 Käthe-Kollwitz-Gymnasium in Zwickau (Germany)

\section{internships and work experience:}

2011 scientific assistent in microbial biotechnology with Prof. S.-E. Behrens at the Martin-Luther-University Halle/Saale

2010-2011 diploma thesis in microbial biotechnology with Prof. S-.E. Behrens at the Martin-Luther-University Halle/Saale

internship at BioSolutions Halle/Saale $\mathrm{GmbH}$

2009 student helper in the Geobotany institute with Prof. H. Bruelheide at the Martin-Luther-University Halle/Saale

2008-2009 internship and student helper in developmental genetics of Prof. G. Reuter at the Martin-Luther-University Halle/Saale

Language skills: $\quad$ English, German 


\section{Publications related to thesis work}

Tauber, M., and Fischle, W. (2015). Conserved linker regions and their regulation determine multiple chromatin-binding modes of UHRF1. Nucleus 6, 123-132. doi: 10.1080/19491034.2015.1026022

Gelato, K.A., Tauber, M., Ong, M.S., Winter, S., Hiragami-Hamada, K., Sindlinger, J., Lemak, A., Bultsma, Y., Houliston, S., Schwarzer, D., et al. (2014). Accessibility of Different Histone H3-Binding Domains of UHRF1 Is Allosterically Regulated by Phosphatidylinositol 5-Phosphate. Molecular Cell 54, 905-919. doi: 10.1016/j.molcel.2014.04.004 


\section{Conserved linker regions and their regulation determine multiple chromatin-binding modes of UHRF1}

Maria Tauber and Wolfgang Fischle*

Laboratory of Chromatin Biochemistry; Max Planck Institute for Biophysical Chemistry; Göttingen, Germany
Keywords: allosteric, chromatin, cooperative, DNA methylation, domains, epigenetic, histone, modifications, multivalent, multiple protein conformations, UHRF1

*Correspondence to Wolfgang Fischle; Email: wfischl@gwdg.de

Submitted: 01/20/2015

Revised: 02/25/2015

Accepted: 03/02/2015

http://dx.doi.org/10.1080/19491034.2015.1026022

This is an Open Access article distributed under the terms of the Creative Commons Attribution-NonCommercial License (http://creativecommons.org/ licenses/by-nc/3.0/), which permits unrestricted non-commercial use, distribution, and reproduction in any medium, provided the original work is properly cited. The moral rights of the named author(s) have been asserted.

Extra view to: Gelato, K.G., Tauber, M., Ong, M. Winter, S., Hiragami-Hamada, K., Sindlinger, J., Lemak, A., Bultsma, Y., Houliston, S., Schwarzer, D., Divecha, N., Arrowsmith, C.H. and Fischle, W. (2014) Interaction of UHRF1 with the unmodified or lysine 9 trimethylated H3 tail is allosterically regulated by phosphatidylinositol 5-phosphate Mol. Cell, 54:905-919. doi: 10.1016j.molcel.2014.04.004. PMID:24813945
$\mathrm{U}$ biquitin-like with PHD and RING Finger domains 1 (UHRF1) is an important nuclear protein that is mutated and aberrantly expressed in many tumors. The protein integrates different chromatin modifications and is essential for their maintenance throughout the cell cycle. Separate chromatinbinding modules of UHRF1 have been studied on a functional and structural level. The unmodified N-terminus of histone $\mathrm{H} 3$ is recognized by a PHD domain, while a TTD domain specifically interacts with histone $\mathrm{H} 3$ Lysine 9 trimethylation. A SRA region binds hemimethylatd DNA. Emerging evidence indicates that the modules of UHRF1 do not act independently of each other but establish complex modes of interaction with patterns of chromatin modifications. This multivalent readout is regulated by allosteric binding of phosphatidylinositol 5phosphate to a region outside the PHD, TTD and SRA domains as well as by phosphorylation of one of the linker regions connecting these modules. Here, we summarize the current knowledge on UHRF1 chromatin interaction and introduce a novel model of conformational transitions of the protein that are directed by the flexible and highly charged linker regions. We propose that these are essential in setting up defined structural states of the protein where different domains or combinations thereof are available for binding chromatin modifications or are prevented from doing so. Lastly, we suggest that controlled tuning of intramolecular linker interactions by ligands and posttranslational modifications establishes a rational framework for comprehending UHRF1 regulation and putatively the working mode of other chromatin factors in different physiological contexts.

\section{Introduction}

DNA methylation is an important epigenetic modification to establish and maintain cell-type-specific gene expression profiles as well as to ensure genome stability. In mammals, the majority of genomic DNA methylation occurs on the 5-position of cytosine bases within CpG dinucleotides. Within the short palindromic sequence DNA nucleotide methyltransferases (DNMTs) modify both strands (so called symmetric or full methylation). CpG methylation (meCpG) does not operate in isolation but is closely interconnected with specific repressive histone Lysine methylation marks and in particular histone H3 Lysine 9 trimethylation (H3K9me3). Histone modifications seem to be involved in targeting DNMTs to specific regions of the genome for regional methylation of DNA. In turn, DNA sequence and methylation state have a great influence on the Lysine methylation status of histones. The interplay between both epigenetic marks and how these are established is depending on the physiological and developmental cellular context. Since both modifications are associated with transcriptional repression and heterochromatin formation, their faithful duplication and transmission during cell division is crucial to preserve genome integrity (reviewed in ref.1)

Ubiquitin-like with PHD and RING Finger domains 1 (UHRF1) is a nuclear factor essential for the maintenance of meCpG and putatively $\mathrm{H} 3 \mathrm{~K} 9 \mathrm{me} 3$ patterns during replication. The protein is 
preferentially localized to pericentric heterochromatin but can also be found in euchromatic regions. ${ }^{2-4}$ Via multiple chromatin binding domains UHRF1 targets enzymes catalyzing meCpG and H3K9me3, DNMT1, DNMT3a/b and G9a, to genomic loci. ${ }^{5-8}$ Since the protein besides maintenance of epigenetic marks is also implicated in gene expression regulation, particularly in silencing of tumor suppressor genes, understanding how it affects chromatin regulatory processes through histone and DNA methylation is of great interest. ${ }^{2,9,10}$

Based on results of functional and structural studies, we recently proposed a role of phosphatidylinositol 5-phosphate $(\mathrm{PI}(5) \mathrm{P})$ in allosteric regulation of UHRF1 binding to $\mathrm{H} 3 \mathrm{~K} 9 \mathrm{me} 3 .^{11}$ Here, we summarize findings that indicate multiple conformational states of UHRF1 that expose different domains providing distinct overall chromatin-binding properties. We suggest these different states of UHRF1 are dependent on conserved linker regions. Lastly, we provide rationale how the different interaction modes of the protein with chromatin might be regulated by the interplay of allosteric ligands, posttranslational modifications and protein binding partners.

\section{Domain structure of UHRF1}

Human UHRF1 is also referred to as ICBP90, while the mouse ortholog is known as Np95. Most vertebrates also contain a highly similar factor, UHRF2 (mNp97). Since hUHRF1 has been most extensively studied, we will, unless otherwise stated, refer to this protein in our discussions. The five conserved domains of UHRF1 (Fig. 1) comprise an N-terminal ubiquitin-like domain (UBL), which is also referred to as NIRF_N (novel Np95/ ICBP90-like RING finger protein N-terminus). This domain has the classic $\alpha / \beta$ ubiquitin fold and contains conserved surface Lysines K31 and K50, which are putative targets of mono- or poly-ubiquitination (pdb entry 2FAZ, unpublished structure). While UHRF1 protein function and/or proteasomal protein turnover might be regulated by the UBL domain, the exact biological role of this region is not yet fully understood. ${ }^{12}$
At the C-terminus of UHRF1 is a Really Interesting and New Gene (RING) domain that has E3 ubiquitin ligase activity. It is composed of 2 zinc-fingers and a novel, unique $\alpha$-helix bundle structure, which is formed by 3 helices localized upstream and one helix localized downstream of the Zn-fingers (pdb entry 3FL2, unpublished structure). According to a recent report the RING domain establishes histone $\mathrm{H} 3$ Lysine 23 (H3K23) monoubiquitination during $S$-phase. It was proposed that this mark serves as a prerequisite for recruitment of the maintenance DNA methyltransferase DNMT1 to target sites of UHRF $1 .{ }^{13}$

Three central domains of UHRF1 are implicated in specific recognition of chromatin modifications, the unmodified $\mathrm{N}$ terminal region of histone $\mathrm{H} 3$ ( $\mathrm{H} 3$ unmod $(\mathrm{N}$-term)), the $\mathrm{H} 3 \mathrm{~K} 9 \mathrm{me} 3$ mark and hemi-methylated DNA $\quad\left(\mathrm{me}_{1 / 2} \mathrm{CpG}\right)$ (Fig. 1). The tandem tudor domain (TTD) is composed of 2 subdomains $\left(\mathrm{TTD}_{\mathrm{N}}: \mathrm{TTD}_{\mathrm{C}}\right)$ that are tightly packed together and that both have a typical tudor family 5 -stranded $\beta$-barrel fold. ${ }^{14}$ An aromatic cage that is built by residues F152, Y188 and Y191 of $\mathrm{TTD}_{\mathrm{N}}$ recognizes diand tri-methylated Lysine residues. Specificity for binding $\mathrm{H} 3 \mathrm{~K} 9 \mathrm{me} 3$ is provided by a peptide-binding groove formed between the 2 individual tudor domains establishing specific and tight contacts to the residues upstream and downstream of the methylated Lysine. Binding of the TTD to an $\mathrm{H} 3 \mathrm{~K} 9 \mathrm{me} 3$ peptide is not sensitive to adjacent Serine 10 phosphorylation $(\mathrm{H} 3 \mathrm{~S} 10 \mathrm{ph}) .^{14,15}$ In contrast, H3 Lysine 4 trimethylation (H3K4me3) and Threonine 6 phosphorylation (H3T6ph) impair affinity for $\mathrm{H} 3 \mathrm{~K} 9 \mathrm{me} 3$. These posttranslational modifications disrupt the important interactions of the $\mathrm{K} 4$ and $\mathrm{T} 6$ side chain and backbone residues with the peptide-binding groove between TTD $_{\mathrm{N}}$ and $\operatorname{TTD}_{\mathrm{C}}{ }^{14}$

The adjacent Plant Homeo Domain (PHD) is a $\mathrm{Zn}$-finger domain; 3 zinc atoms coordinate its rod-shape structure. It recognizes the $\mathrm{N}$-terminus of the $\mathrm{H} 3$ tail solely when unmodified (H3unmod $\left(\mathrm{N}\right.$-term)). ${ }^{2}$ Accordingly, the crystal structure of the PHD with bound H3-tail peptide revealed that Alanine $1(\mathrm{H} 3 \mathrm{~A} 1)$ and Arginine 2 (H3R2) make hydrogen bond- contact to specific residues of the domain. ${ }^{16}$ Remarkably, the first zinc atom coordinates a loop, the so-called prePHD that precedes the canonical PHD-fold. This structural feature was first identified in the UHRF1 PHD domain and its detailed function still needs to be determined. ${ }^{16,17}$ It was suggested that the prePHD might be essential for the right orientation of residue $\mathrm{C} 316$, which makes contact with $\mathrm{H} 3 \mathrm{~K} 4 .{ }^{16}$ However, if analyzed in isolation the PHD does not much discriminate the modification status of H3K4. In contrast, phosphorylation of H3 Threonine 3 (H3T3ph), symmetric as well as asymmetric dimethylation of H3R2 (R2me2s/a) and H3A1 N-terminal acetylation (H3A1ac) strongly interfere with binding of the PHD to the H3-tail. ${ }^{2}$

C-terminal to the PHD is a SET and RING-associated domain (SRA). It consists of a $\beta$-barrel flanked by $\alpha$-helical elements forming a half moon-like structure with a basic inner surface. ${ }^{18}$ Two loops sticking out of this structure grasp into the major and the minor groove of DNA. R491 is part of the so-called N-K-R finger, which specifically forms hydrogen bonds with $\mathrm{CpG}$ sequences. These contacts flip out a cytosine from the double helix placing it in a binding pocket that is tailored for the recognition of 5-methylcytosine $(5 \mathrm{mC}) .{ }^{19}$ In this pocket $5 \mathrm{mC}$ is sandwiched by stacking interactions with 2 aromatic residues (Y478, Y466). The SRA domain exhibits significant specificity for hemi-methylated DNA $\left(\mathrm{me}_{1 /}\right.$ ${ }_{2} \mathrm{CpG}$ ). This is facilitated by N489, which is a part of the N-K-R finger and makes contacts to the non-methylated adverse cytosine on the second DNA strand. Methylation of this cytosine disturbs positioning of the N-K-R finger and therefore impairs SRA binding. ${ }^{18}$

\section{Interplay of UHRF1 chromatin- binding domains}

Given that UHRF1 contains 3 separate domains that can recognize different chromatin modifications, the question arises whether these work independently or in concert. The combinatorial interaction of different domains of one protein with a complex target containing multiple ligands is generally referred to as multivalent binding. Several chromatin factors 


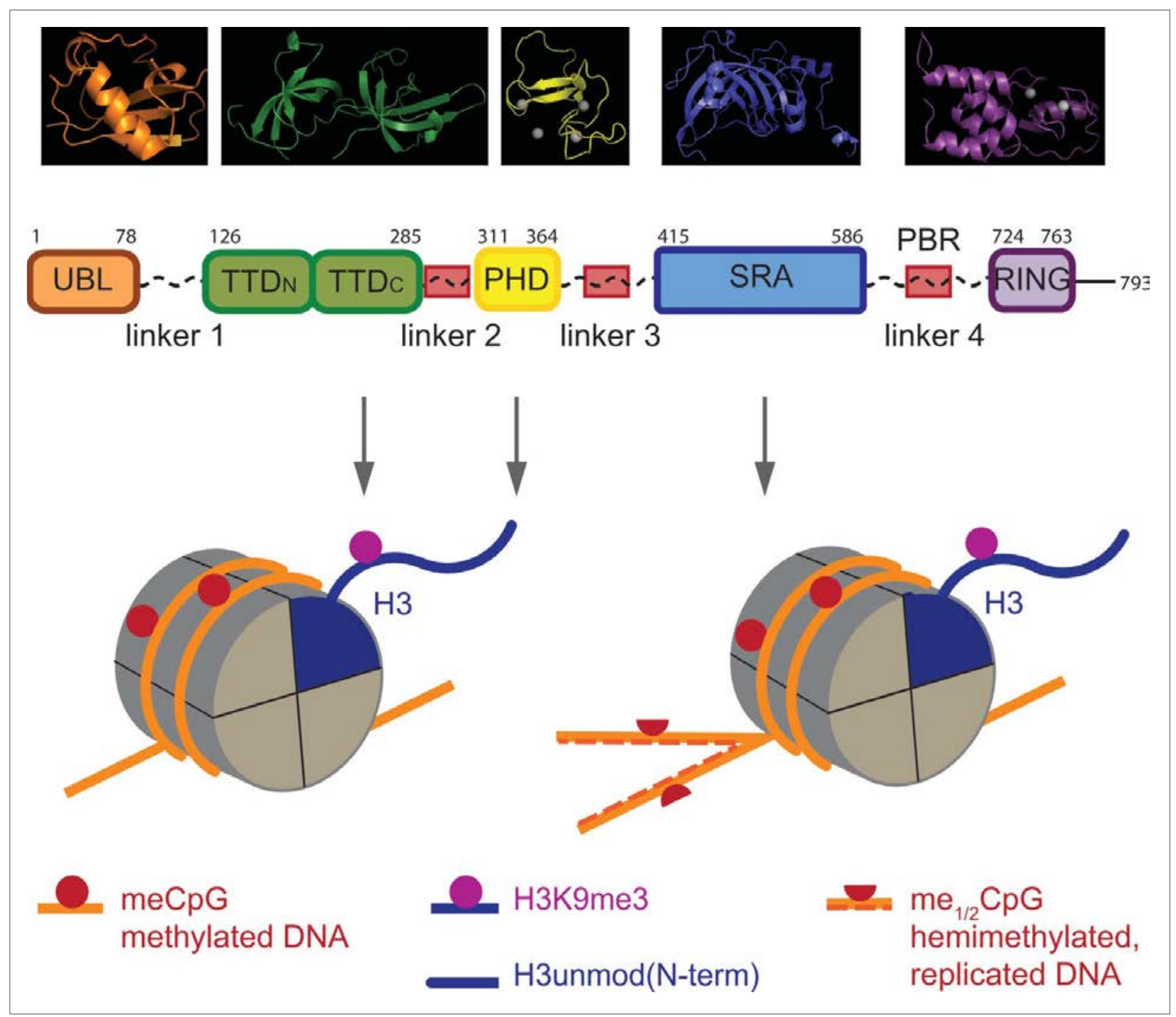

Figure 1. UHRF1 is a multi-domain factor with several conserved protein motifs connected by linker regions. Schematic representation of the domain structure of human UHRF1 isoform 1 is shown (UniProtKB: Q96T88). Top: The structures of the individual domains of UHRF1 as determined by X-ray crystallography and NMR spectroscopy visualized and rendered using MacPyMOL v 1.7.0.3 (pymol.org). Middle: Domain boundaries are given by respective starting and ending amino acid positions. Conserved stretches of low complexity and high content of basic amino acids within the linker regions are indicated (red boxes). Bottom: Chromatin ligands of the different domains of UHRF1; schematic representation of a single nucleosome in interphase (left) and immediately after replication in S-phase (right). For simplicity only one H3-tail is shown. UBL, ubiquitin like; TTD, tandem tudor domain; PHD, plant homeodomain; SRA, SET and RING associated; PBR, polybasic region; RING, really interesting and new gene. pdb entries are: UBL, $2 F A Z ; T^{2} D_{N}: T T D_{C}$, 3DB3; PHD, 3SHB; SRA, 2PB7; RING, $3 F L 2$.

such as TRIM24 and BPTF have been shown to recognize patterns of histone modifications in a multivalent mode. ${ }^{20,21}$ In these proteins different binding domains simultaneously engage in interaction with separate histone modifications Such cross-talk in interaction on the same or distinct histone tails is considered a fundamental element of the so-called histone code theory that postulates that patterns of chromatin marks constitute a complex signaling system. ${ }^{22}$ To this point, multivalent chromatin interactions have been rationalized in a static manner, with recognition domains acting independently of each other. ${ }^{23,24}$ Several observations indicate that multivalent binding of UHRF1 to chromatin is more complex.

TTD-PHD interplay in recognizing the $\mathrm{H} 3$-tail

Different biophysical studies have quantified the interaction strength of the isolated TTD of UHRF1 with
H3K9me3 peptides. Depending on the experimental conditions the dissociation constant $\left(\mathrm{K}_{\mathrm{D}}\right)$ was determined within a range of $1.0 \mu \mathrm{M}$ to $2.5 \mu \mathrm{M} .^{11,15,25,26}$ Similarly, binding of the isolated PHD domain to H3unmod(N-term) was mapped at a $\mathrm{K}_{\mathrm{D}}$ of $0.7 \mu \mathrm{M}$ to 2.5 $\mu \mathrm{M}^{2,17,26,27}$ In contrast, a UHRF1 TTD-PHD cassette showed around 5fold enhanced binding $\left(K_{D}\right.$ between $0.15 \mu \mathrm{M}$ and $0.5 \mu \mathrm{M}$ ) to a histone $\mathrm{H} 3$ peptide containing both, an unmodified 
N-terminus and the K9me3 mark, implying a multivalent binding modus. ${ }^{11,25,26,28}$

Since the H3-tail is embedded in the peptide-binding groove of the TTD in the isolated structure of this complex, how can the PHD get access to the ultimate Nterminus of $\mathrm{H} 3$ in the context of the TTD-PHD cassette? Fluorescence/Förster Resonance Energy Transfer (FRET) experiments revealed a conformational shift induced in the TTD-PHD cassette upon interaction with the $\mathrm{H} 3$-tail. ${ }^{25}$ In agreement, structural studies using cocrystalization as well as NMR measurements indicate that the H3-binding mode of the TTD-PHD cassette is different from that of the isolated TTD (Fig. 2A). Obviously, the short region connecting TTD and PHD (linker 2, Fig. 1) replaces the H3tail from the peptide-binding groove of the TTD and itself occupies this interface. The resulting arrangement has the $\mathrm{H} 3$-tail connecting the PHD, which binds the ultimate N-terminus, with the TTD, which binds K9me3. Two Arginine and a Lysine residue (R295-R296-K297) in linker 2 are crucial for stabilizing this TTD-PHD conformation. Indeed, mutation of R295 and R296 results in loss of multivalent binding. ${ }^{26,28}$ In contrast, dynamic NMR studies indicate multiple modes of PHD linkage in relation to linker 2 and TTD. ${ }^{28}$ Also, the TTDPHD cassette only crystalized in presence of the H3K9me3-tail peptide. ${ }^{26}$ The findings imply that the 2 domains do not directly interface but that their relative localization is variable without ligand.

The reduced binding surface of the TTD with the H3-tail in the TTD-PHD/ $\mathrm{H} 3 \mathrm{~K} 9 \mathrm{me} 3$ complex as well as the flexible linkage of the ligand and between the domains explain the observed relative weak enhancement of interaction by the multivalent binding mode. Indeed, the PHD domain is dominating the interaction of the TTD-PHD cassette with the $\mathrm{H} 3 \mathrm{~K} 9 \mathrm{me} 3$-tail. If binding of the PHD to the H3-tail is abolished by mutation of D334 or modification of the N-terminus of the $\mathrm{H} 3$ peptide (A1ac, T3phos), the binding of the neighboring TTD to methylated $\mathrm{K} 9$ is drastically weakened or completely lost. Conversely, PHD interaction with the unmodified $\mathrm{N}$-terminus of
H3 in the context of the PHD-TTD cassette is largely unaffected by mutation of the $\mathrm{TTD}_{\mathrm{N}}$ aromatic cage (F152, Y188) or histone modifications such as $\mathrm{H} 3 \mathrm{~K} 4 \mathrm{me} 3$, which abolish the binding of the isolated TTD to the H3-tail. ${ }^{15,25,26,28}$

\section{Control of TTD/H3K9me3 interaction by a C-terminal polybasic} region

While reductionistic approaches studying isolated domains and combinations thereof have provided detailed insights into the chromatin interaction potential of UHRF1, other studies looked directly at the full-length protein. Here, striking differences in interaction specificity for the $\mathrm{H} 3$-tail were observed. While native UHRF1 analyzed in the context of mammalian cell extracts shows clear preferences for $\mathrm{H} 3 \mathrm{~K} 9 \mathrm{me} 3$, the recombinant protein expressed in bacteria or insect cells binds similarly to unmodified $\mathrm{H} 3$ and H3K9me3 peptides. ${ }^{2-4,11,29}$ We recently showed that this discrepancy is due to intramolecular interaction of the TTD with a polybasic region (PBR) located between the SRA and RING domains of UHRF1 (Fig. 2B). ${ }^{11}$ Our studies imply that in the absence of ligands (i.e. the recombinant purified protein) the PBR but not linker 2 occupies the peptidebinding groove of the TTD. This prevents interaction with $\mathrm{H} 3 \mathrm{~K} 9 \mathrm{me} 3$, whereas the PHD is unaffected and can bind the ultimate unmodified H3-tail.

NMR experiments have revealed that a K-R-K motif (K648-R649-K650) is essential for placing the PBR in the peptidebinding groove of the TTD surface. Competition experiments with isolated domains indicate that this interaction is stronger than the similar TTD-linker 2 interplay. It also fully blocks the binding of the $\mathrm{H} 3 \mathrm{~K} 9 \mathrm{me} 3$-tail. In the context of the recombinant, full-length protein mutagenesis of the K-R-K motif is necessary to release the PBR from the TTD. ${ }^{11}$ The resulting mutant UHRF1 protein appears to be in an intermediate state as the TTD and PHD both can bind their respective $\mathrm{K} 9 \mathrm{me} 3$ and H3unmod(N-terminus) ligands.

Dialysis of recombinant UHRF1 against nuclear extract isolated from HeLa cells induced yet another UHRF1 binding state. Interestingly, this form resembles the native cellular protein in preference for $\mathrm{H} 3 \mathrm{~K} 9 \mathrm{me} 3$ over $\mathrm{H} 3 \mathrm{~K} 9 \mathrm{me} 0$ (Fig. 2C). ${ }^{11}$ The results not only infer an allosteric regulatory mode of UHRF1, but also indicate that states of the protein exist where the PHD domain is prevented from binding $\mathrm{H} 3$ unmod(N-term).

\section{Multivalent binding to histone $\mathrm{H} 3$ and DNA methylation}

The isolated SRA domain of UHRF1 binds $\mathrm{me}_{1 / 2} \mathrm{CpG}$ with a dissociation constant of around $200 \mathrm{nM} .^{30}$ Of the 3 chromatin-binding domains it therefore has the tightest interaction with a ligand. While life cell imaging and fluorescence recovery after photobleaching have revealed that the SRA domain dominates targeting of $\mathrm{mNp} 95$ to pericentromeric heterochromatin in vivo, mutagenesis studies imply that the PHD and/or TTD domains are also required in this context. ${ }^{29}$ Indeed, in vitro binding experiments with $\mathrm{mNp} 95$ protein isolated from cells show that presence of histone $\mathrm{H} 3 \mathrm{~K} 9 \mathrm{me} 3$ peptide promotes interaction of the SRA with hemi-methylated DNA. Conversely, interaction with histone peptides is enhanced in presence of un-/methylated DNA. ${ }^{31}$ While the mechanistic details of this interplay have not yet been unveiled, the findings suggest that the SRA domain cooperates with the TTD and/or PHD in binding to multiple-modified chromatin targets.

\section{A conformational transition model for UHRF1 chromatin binding}

It has not been possible to deduce a simple, coherent picture of UHRF1 multivalent chromatin binding on the basis of the research on cassettes composing more than one of the chromatin binding domains, our molecular analysis of the full-length protein as well as based on the multiple studies of deletion and point mutants of the protein in recombinant form, extracted from cells, or in different cellular context (see for example refs.3,29). A putative explanation might come from the idea that the TTD, PHD, and SRA (and possibly UBL and RING) domains do not work independently. Indeed, we favor the view that engagement of the different binding domains of UHRF1 with ligands influences the 


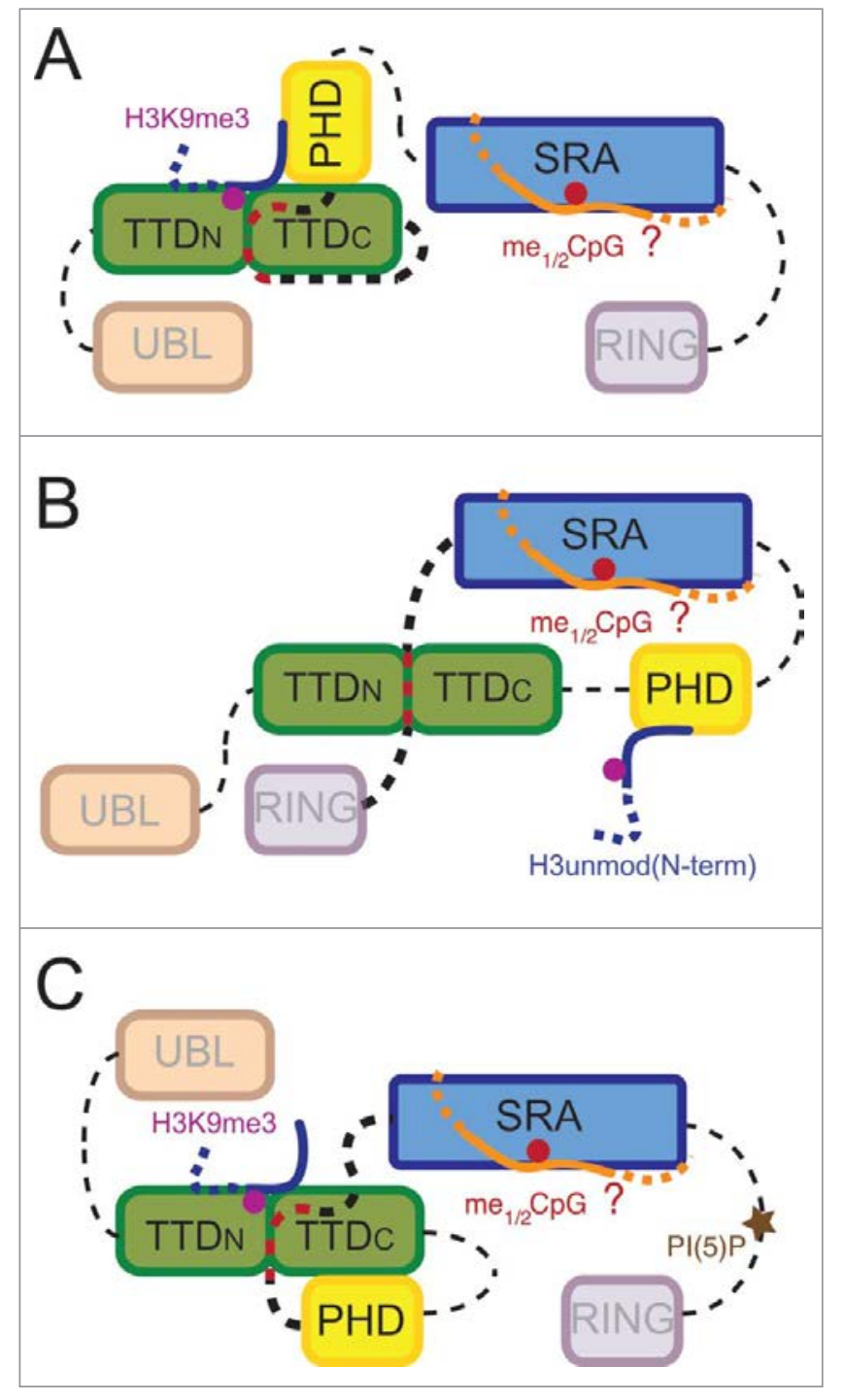

Figure 2. Conformational transition model for UHRF1 binding to chromatin marks. The three conformational states shown (A, B, C) correspond to binding modes deduced by different studies. While some parameters of domain and ligand interaction are known, others are hypothetical. For example, the exact coupling of $\mathrm{H} 3 \mathrm{unmod}\left(\mathrm{N}\right.$-term), $\mathrm{H} 3 \mathrm{~K} 9 \mathrm{me} 3$, and $\mathrm{me}_{1 / 2} \mathrm{CpG}$ binding is not known. Also, the mechanism of PHD inhibition in state (C) has not been resolved. Additional other binding states and relative orientations of the domains might exist. For representation purposes, linker regions are not drawn to scale. Linker regions that are involved in setting up different conformational states via interaction with the peptide-binding groove of the TTD are highlighted. Note that the role of linker 3 has not been determined. Binding of the allosteric regulator PI(5)P to the PBR region releases linker 4 from the TTD peptide-binding groove.

interaction properties of each other. Since the structural analysis of the isolated domains has not indicated any conformational changes of the binding pockets induced by ligand, the cooperative mode of interaction must be mediated on another level. We suggest that UHRF1 exists in multiple protein conformations where different, structurally invariable binding domains or combinations thereof are either exposed and available for interaction with chromatin marks or where these are occluded and prevented from ligand binding (Fig. 2). We postulate that these conformational states are in constant exchange with each other and that the actual equilibrium between the distinct forms determines the apparent binding properties of UHRF1.
Conserved linker regions likely establish different UHRF1 conformational states

How are different conformational states of UHRF1 established? The regions connecting the conserved and easily recognized chromatin modification-binding domains might play a major role (Figs. 1 and 2). These contain the linker 2 between the TTD and PHD (26 aa in hUHRF1), linker 3 between the PHD and SRA (51 aa in hUHRF1), and the PBR containing region between the SRA and RING (linker 4, 138 aa in hUHRF1). Algorithms that predict secondary structures fail to assign particular folds to these regions. The linkers might therefore form random, intrinsically disordered structures.

Despite the lack of conserved folds, short (ca. 20 aa) sequence stretches are highly conserved within linker 2, linker 3 and PBR (Fig. 3A). For example, the region between aa 372 and 391 of linker 3 shows sequence identity of around $90 \%$ in all analyzed UHRF1 proteins. ${ }^{12}$ Besides the conserved sequences, these stretches of the linker regions are of relative low complexity, enriched in basic amino acids (i.e., Lysine and Arginine residues). This seems to be functionally important as for hUHRF1 the R-R-K element in linker 2 and the K-R-K element in the PBR have been shown to be crucial for binding of these regions to the peptide-binding groove of the TTD. ${ }^{11,26,28}$ Since the interacting polar residues on the surface of the TTD domain also exhibit conservation throughout all analyzed species (Fig. 3B), the competition of linker 2 and PBR for binding the TTD seems to constitute a major element for establishing different UHRF1 conformational states. Due to the high sequence conservation and a central K-K-K element we further hypothesize that linker 3 might also interface with the peptide-binding groove of the TTD (Fig. 2C). We think that depending on which linker associates with the TTD, different overall conformations of UHRF1 are established. Lastly, we theorize that other regions of UHRF1 might contain polar surfaces similar to the peptide-binding groove of the TTD for accommodating linker 2, linker 3 or $\mathrm{PBR}$ in the different conformational states. 


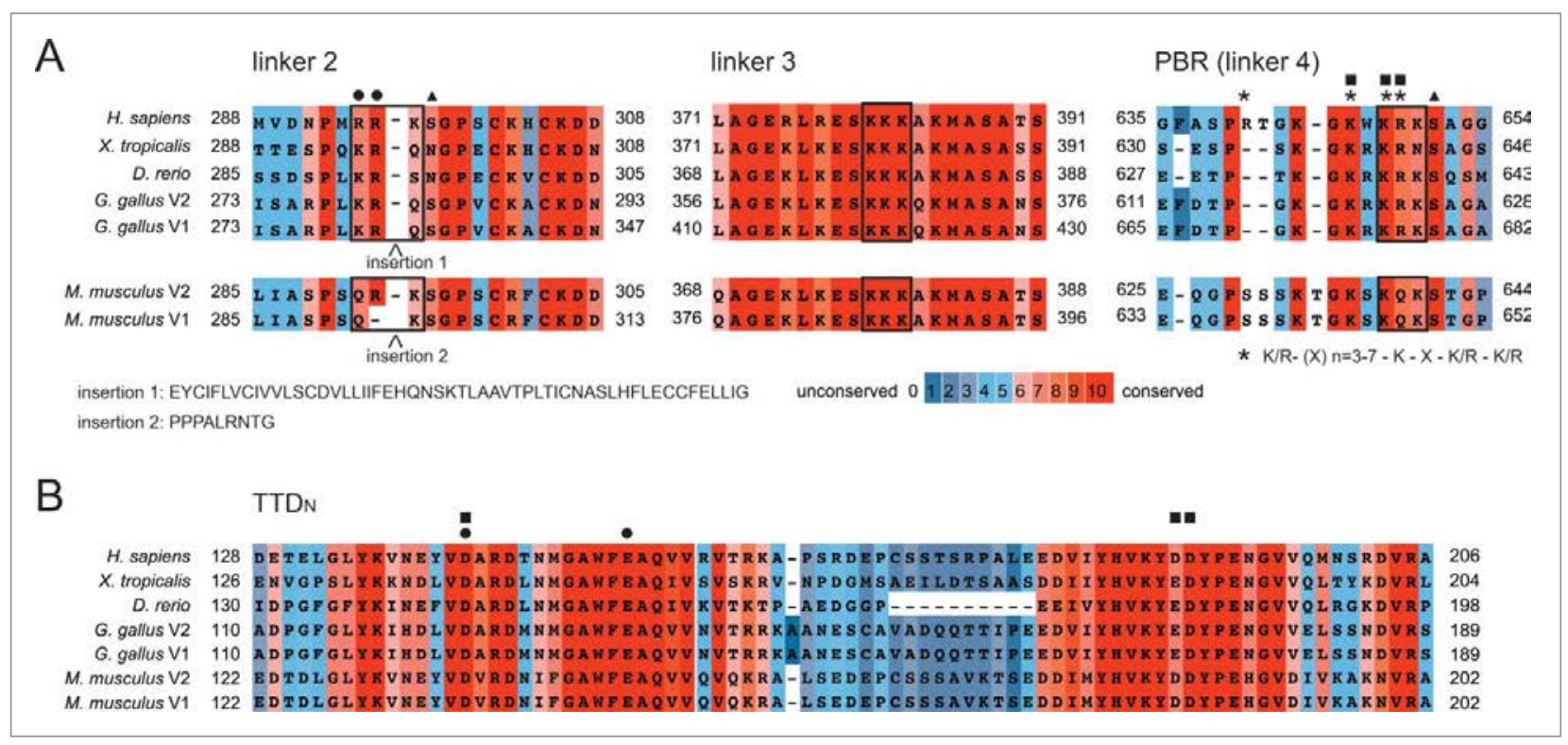

Figure 3. The different linker regions and the peptide binding-groove of the TTD are highly conserved. Multiple sequence alignments of sequence stretches within the linker regions (A) and the peptide-binding groove of the TTD (B) of UHRF1. Alignments were prepared using the PRALINE alignment tool at http://www.ibi.vu.nl/programs/pralinewww/. Numbers correspond to amino acid positions at the beginning and end of the respective protein sequences of the different species. Sequences of insertions found in splicing variants of mouse and chicken proteins are given. Symbols indicate residues implied in the interaction of the TTD peptide-binding groove with linker 2 (dots) or the PBR region within linker 4 (squares). Triangles mark known sites of phosphorylation. Asterisks mark positions corresponding to a putative PIP binding consensus motif within the PBR. Boxes highlight elements of basic residues found in linkers 2, 3 and 4. UHRF1 amino acid sequences according to NCBI: Homo sapiens, NP_001276981.1; Mus musculus v1, NP_001104550.1, Mus musculus v2, NP_001104548.1, Gallus gallus v1, XP_418269.4, Gallus gallus v2, XP_004949013.1; Xenopus laevis, F6UA42.2; Danio rerio, NP_998242.1.

As linker 2 and its interaction with the peptide-binding groove of the TTD might be elementary in setting up distinct conformational states of UHRF1, it is interesting to note that in mouse as well as in chicken splicing variants of the protein exist that only differ in this region. Even more perplexing is the fact that both insertions occur right at the R-R-K element important for the interaction with the peptide-binding groove of the TTD (Fig. 3A). While the linker 2 of $\mathrm{mNp} 95$ variant 2 is very similar to the corresponding region of the single human UHRF1 protein, variant 1 contains an insertion of 9 aa, which eliminates the first Arginine of the motif. And whereas the chicken sequences are only predicted but lack experimental validation, variant 1 of this organism has a 54 aa insertion just ahead of the R-R-K element.

Unfortunately, work on mNP95 did so far not discriminate between variants 1 and 2 (note that chicken UHRF1 has not yet been studied). Nevertheless, careful reevaluation of the available data indicates that the linker insertion in variant 1 might block multivalent binding of the TTDPHD cassette to the H3K9me3-tail. ${ }^{31} \mathrm{We}$ therefore think that the combinatorial binding of both domains is strongly dependent on the sequence and length of linker 2 that could alter the spacing and orientation of the TTD and PHD. On the basis of our conformational transition model of UHRF1, we predict that certain states are not or less populated by the different splicing variants of UHRF1 in mouse and chicken. These should therefore have non-overlapping roles. Detailed analysis of the expression and function of the splicing variants in mouse and/or chicken might be a fruitful avenue for further dissecting the molecular working mode and regulation of UHRF1.

\section{Regulation of UHRF1 conformational states}

While intrinsically disordered, the linker regions might nevertheless adopt a series of limited, defined transitional states enabling interaction with different polar surfaces of UHRF1 and determining different conformational and binding states.
The kinetic barrier for these transitions could be relatively low due to the intrinsic linker flexibility. As consequence, the protein might be able to rapidly change conformations in response to external stimuli and structural perturbations. This in turn would facilitate changes in the population of certain states therefore affecting UHRF1 target binding and cellular function. $^{32}$

Different events might affect the equilibrium of the conformational states of UHRF1. If forms of the protein exist that enable more than one of the chromatin binding domains to interact with their ligand (Fig. 2), engagement of one of the exposed regions with its target will facilitate a secondary binding event of the other available domain in a cooperative fashion. While we do not (yet) know how many different forms and binding states of UHRF1 might exist, such interpretation is in full agreement with the observed enhancement of $\mathrm{H} 3 \mathrm{~K} 9 \mathrm{me} 3$-tail binding of mNP95 by DNA and vice versa. ${ }^{31}$

Allosteric ligands provide another level of regulating UHRF1 conformational 
states. Based on our observations that recombinant UHRF1 behaved differently from cellular protein but could be converted to a state with similar H3K9me3binding properties after dialysis against nuclear extract, we biochemically defined the cofactor and regulator as phosphatidylinositol 5-phosphate (PI(5)P). ${ }^{11}$ PIPs are amphiphilic glycerophospholipids that consist of a polar inositol head group linked by a phosphodiester bridge to a glycerol backbone branching 2 non-polar fatty acids (Fig. 4). PI(5)P is a low abundance PIP present primarily in mammalian cytoplasmic cell membranes with a smaller population in the cell nucleus. ${ }^{33}$ While its nuclear functions are not fully clear, its presence in this compartment is highly conserved throughout eukaryotes. ${ }^{34-36}$ We could show that PI(5)P binding to UHRF1 specifically requires the $\mathrm{PBR}$ region and that this interaction releases the latter from the peptide-binding groove of the TTD. In consequence, a conformation of UHRF1 is established that allows the TTD to bind $\mathrm{H} 3 \mathrm{~K} 9 \mathrm{me} 3$ independent of the PHD. ${ }^{11}$

While we do not yet know how exactly $\mathrm{PI}(5) \mathrm{P}$ is bound by UHRF1 - the protein has to accommodate both, the polar head group as well as the hydrophobic acyl chains - it is interesting to note that the PBR resembles in sequence a previously defined consensus PIP interaction motif $\left(\mathrm{R} / \mathrm{K}-\left(\mathrm{X}_{\mathrm{n}}=3-7\right)-\mathrm{K}-\mathrm{X}-\mathrm{K} / \mathrm{R}-\mathrm{K} / \mathrm{R}\right)$ shared by several PIP interacting factors. ${ }^{37}$ Given that the linker 2 and linker 3 are also highly conserved and enriched in basic amino acids, we are wondering whether other ligands modulate the behavior of these regions. We think this is an idea worth entertaining, particularly since synthetic PI(5)P did not fully recuperate all changes of recombinant UHRF1 observed after dialysis against cellular extract. ${ }^{11}$

The transitions between different conformational states of UHRF1 1 conveyed by the linker regions might be further regulated by posttranslational modifications (PTM). These could impair binding to the peptide-binding groove of the TTD or putatively other regions of the protein (Fig. 4). Indeed, it was shown previously that phosphorylation of a Serine residue (S298) adjacent to R295-R296-K297, decreases the binding affinity of the TTDPHD cassette for the H3K9me3-tail. The altered binding stoichiometry of the complex indicates that the coupling of TTD and PHD mediated by linker 2 is impaired by the PTM. ${ }^{26}$

A number of additional sites of Serine phosphorylation and other posttranslational modification have been identified in UHRF1 by proteomics studies. Some of these map to highly conserved residues within the linker 2, linker 3 and PBR regions (Fig. 3A). ${ }^{38-43}$ These events have the potential to regulate UHRF1 conformational states. For example, phosphorylation of S651 that is found during human embryonic stem cell differentiation and is next to the K648-R649-K650 element ${ }^{40}$ might impair the interaction of the PBR with the peptide-binding groove of the TTD thereby enhancing a particular UHRF1 chromatin-binding mode.

Finally, we point out that binding of UHRF1 to other proteins such as DNMTs, G9a, USP7, etc. will affect the equilibrium of the various forms of the protein. These interactions require distinct, specific surfaces that will or will not be available in the different conformational states of UHRF1. Conversely, it is expected that engagement of a particular protein partner will stabilize a certain

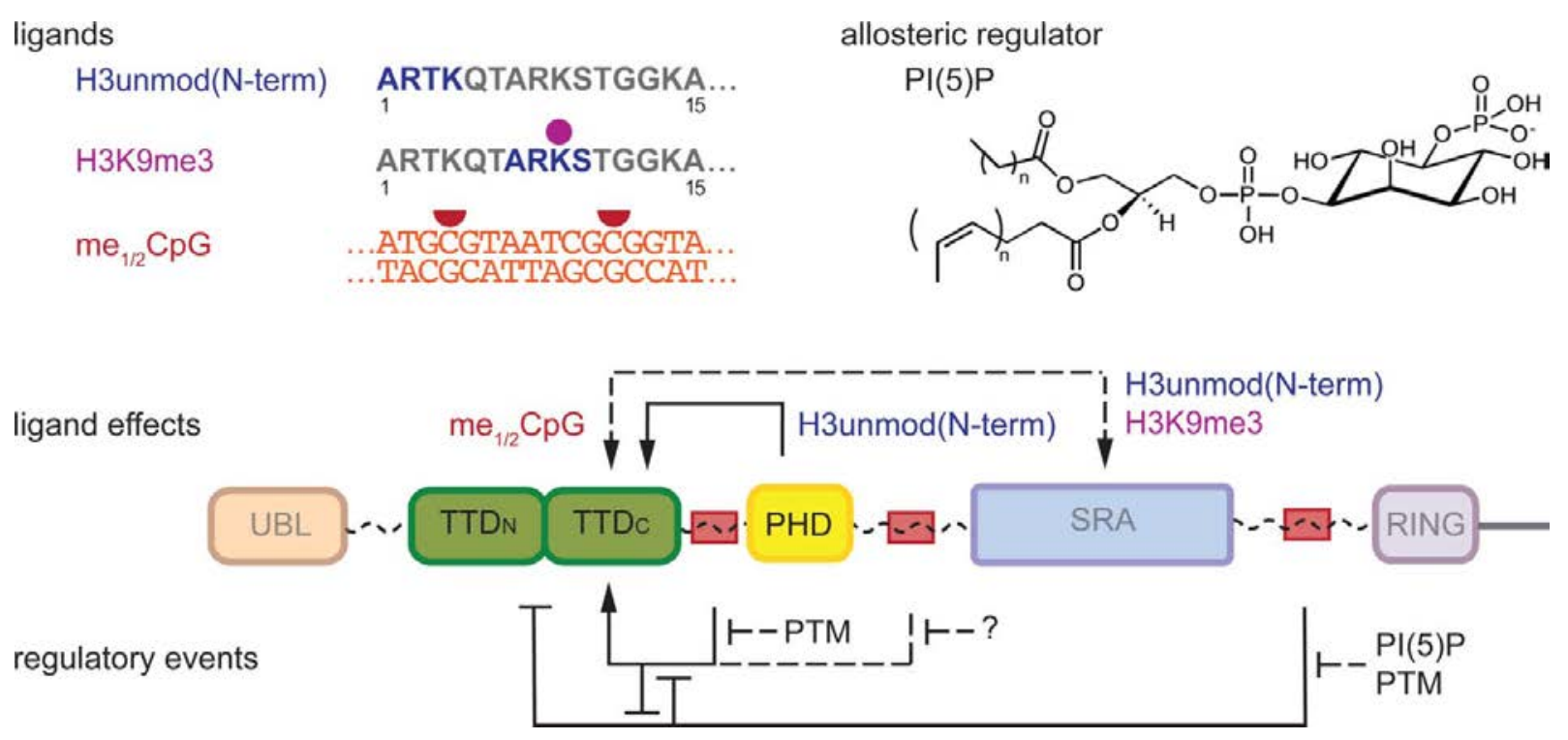

Figure 4. Regulation of the conformational transitions of UHRF1. The interplay of the chromatin-binding domains of UHRF1 and therefore the conformational states of the protein are affected by binding of different ligands, the allosteric regulator phosphatidylinositol 5-phosphate (PI(5)P) as well as posttranslational modifications (PTM) within the linker regions. Enhancing as well as suppressing effects on chromatin modification-binding properties of the TTD, PHD and SRA domains have been described. Dashed lines indicate domain interplay that has not been fully resolved. The regulation of linker 3 is hypothetical (question mark). 
conformation and accompanying chromatin mark-binding mode of UHRF1 (Fig. 4).

Cellular role of the different chromatin binding states of UHRF1

Many studies on UHRF1 have emphasized the importance of the 3 chromatinbinding domains in facilitating specific chromatin association and multiple functions in cellular context. We think that the fact that defined functions could not be pinpointed exclusively to particular domains agrees with extensive collaboration and interplay of these regions. It was shown that the TTD, PHD and SRA domains are all participating in targeting of UHRF 1 to heterochromatin, ${ }^{3,28}$ a process that might be dependent on H3K9me3 and meCpG. The TTD, PHD, and SRA regions are also necessary for recruitment of DNMT1 to replicating foci and thus for proper maintenance of DNA-methylation. ${ }^{3,13,28}$ However, it was also found that UHRF1 binds to promoter regions of euchromatic genes and regulates their expression. Interestingly, these loci lack H3K9me3 but exhibit DNA methylation paired with $\mathrm{H} 3$ unmod (N-term) (i.e. absence of H3R2 symmetric dimethylation). ${ }^{2}$ It is conceivable that UHRF1 binds to these genomic regions by multivalent interaction of the PHD and SRA domains with the unmodified $\mathrm{N}$-terminal H3-tail and methylated DNA, respectively.

Additionally, it was found that UHRF1 interacts with the de novo DNA methyltransferases DNMT3a and DNMT $3 b$ and that this complex mediates epigenetic silencing in a histone $\mathrm{H} 3$ Lysine 9 methyltransferase (i.e., G9a, Suv39h1/2) dependent manner. ${ }^{8}$ Depletion of $\mathrm{Np} 95$ in mouse embryonic stem cells results in severe global DNA hypomethylation. ${ }^{44}$ A similar DNA methylation defect is caused by depletion of all 3 DNMTs $^{45}$ or of histone methyltransferases (G9a, Suv39h1/2). ${ }^{46,47}$ Hence, there is circumstantial evidence that UHRF1 is not only involved in replication-coupled maintenance of DNA methylation, but also in de novo DNA methylation in an H3K9me3-dependent manner. Since de novo methylation has no hemimethylated DNA template, UHRF1 targeting to these chromatin regions might be dependent on interaction of the TTD or TTD-PHD cassette with $\mathrm{H} 3 \mathrm{~K} 9 \mathrm{me} 3$.

Our work showed that manipulation of cellular PI(5)P levels affects UHRF1 subnuclear localization. ${ }^{11}$ Several links between the reported functions of UHRF1 and nuclear PI(5)P levels support the idea of allosteric control of the protein. It was shown previously that nuclear PI(5)P levels are tightly regulated during the cell cycle and in response to certain cellular signaling cascades. PI(5)P levels increase up to 20-fold during transition of G1 - to S-phase. ${ }^{34,48-50}$ Cell cycle analysis of UHRF1 localization in relation to heterochromatic foci indicates changed distribution at the onset of S-phase, ${ }^{51}$ which might be caused by regulation of TTD/ H3K9me3 interaction by $\mathrm{PI}(5) \mathrm{P}$. Also,

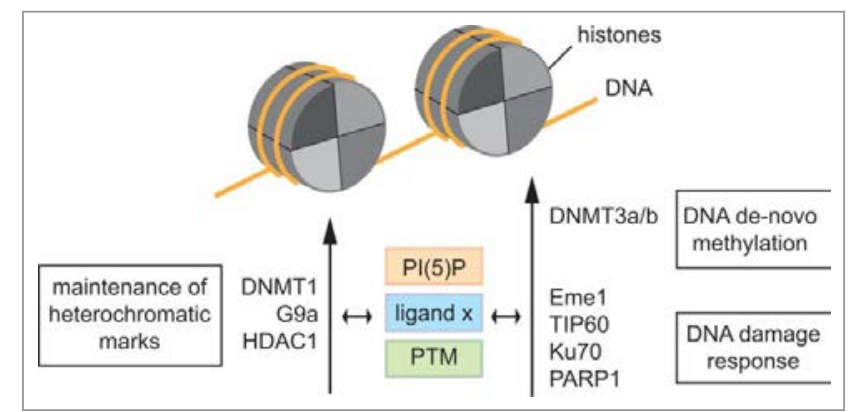

Figure 5. Regulation of interaction of UHRF1 with chromatin and multiple interaction partners. The allosteric regulator phosphatidylinositol 5-phosphate (PI(5)P), different chromatin marks as ligands and posttranslational modifications regulate the postulated different conformations of UHRF1. This impacts on interaction with different protein partners in distinct physiological pathways of chromatin control. Conversely, the protein-binding partners of UHRF1 putatively affect interaction with ligands, allosteric regulators as well as establishment of post-translational modifications.

UHRF1 interacts with several factors associated with the DNA damage response such as Eme1, TIP60, Ku70, and PARP1. ${ }^{44,52-54} \mathrm{PI}(5) \mathrm{P}$ levels are increased after irradiation and exposure to oxidative stress. $^{34,49,50,55}$ Since H3K9me3 has an important role in repair mechanisms of DNA damage, ${ }^{56}$ we speculate that UHRF1 might in a specific context target the repair factors to $\mathrm{H} 3 \mathrm{~K} 9 \mathrm{me} 3$ enriched loci (Fig. 5).

While nothing is known about the in vivo significance of the PTMs of UHRF1 within the linker region, we note that this essential effector protein is incorporated into various complexes involved in different chromatin regulating processes (i.e. maintenance of DNA methylation, de novo DNA methylation, histone $\mathrm{H} 3$ methylation, and histone $\mathrm{H} 4$ deacetylation). ${ }^{7,8,44,51,57,58}$ Thus, it was shown to be an important factor to facilitate DNA replication, cell cycle progression, transcriptional repression, and DNA damage response. $2,53,59-61$ This variety of functions argues for different states of the protein that provide distinct binding interfaces not only for histone and DNA modifications but also for specific protein interaction partners (Fig. 5). 52,53,57,62

\section{Concluding Remarks}

Here, we discussed in detail the modification binding behavior of UHRF1 that reveals novel features of a chromatin binding protein. First of all, the different regions of the protein appear not to function in isolation. We expect that further functional and structural analysis of the full-length protein or of cassettes containing more than one of the TTD, PHD and SRA chromatin-binding domains will uncover that these generally do not interact with their targets independent of each other. We think their interplay induces complex binding modes that cannot simply be explained by static multivalent and simultaneous engagement of different protein surfaces with complex ligands (i.e., key and lock behavior). Instead, UHRF1 might exist in multiple, distinct but constantly exchanging conformations. We propose that these are brought about by flexible linkers that intramolecularly 
compete for a limited number of different sites thereby inducing and stabilizing folds of the protein that expose or occlude the different chromatin-binding interfaces. Besides cooperativity in interaction with chromatin marks, a major consequence of these considerations is a general rationale for comprehending regulation of UHRF1 by allosteric ligands such as $\mathrm{PI}(5) \mathrm{P}$ and posttranslational modifications that both target linker regions outside of the actual chromatin-binding modules.

The transitions between the different conformations and binding states of UHRF1 are dynamically regulated in a physiological context and in response to external stimuli. This in turn controls specific localization of UHRF1 to defined target loci of the genome as well as interaction with different protein partners such as chromatin modifying enzymes. Any perturbation of these regulatory processes will have profound consequences on chromatin structural organization and subsequently lead to developmental aberrations, chromosome instability and oncogenesis - a major outcome of UHRF1 mutation or abnormal expression. ${ }^{63,64}$

UHRF1 might be a paradigm for comprehending chromatin interaction and regulation of other factors in response to cellular internal or external stimuli. We note that other related multi-domain proteins as for example DNMT1 and PARP1 also comprise flexible linkers between their functional domains. Interestingly, these might putatively also interact with PIPs via polybasic regions. ${ }^{37} \mathrm{We}$ expect that future studies will not only reveal a more general influence of small cellular metabolites such as PIPs onto chromatin binding factors but also provide a general framework for the role of interchanging, transient conformational states in protein regulation.

\section{Disclosure of Potential Conflicts of Interest}

No potential conflicts of interest were disclosed.

\section{Acknowledgments}

We thank Dr. Ron Finn for help with protein alignment.

\section{Funding}

This work was supported by the Deutsche Forschungsgemeinschaft (DFG, FI 1513/2-1) and the Max Planck Society.

\section{References}

1. Rose NR, Klose RJ. Understanding the relationship between DNA methylation and histone lysine methylation. Biochim Biophys Acta 2014; 1839:1362-72; PMID:24560929; http://dx.doi.org/10.1016/j. bbagrm.2014.02.007

2. Rajakumara E, Wang Z, Ma H, Hu L, Chen H, Lin Y, Guo R, Wu F, Li H, Lan F, et al. PHD finger recognition of unmodified histone H3R2 links UHRF1 to regulation of euchromatic gene expression. Mol Cell 2011, 43:275-84; PMID:21777816; http://dx.doi.org/ 10.1016/j.molcel.2011.07.006

3. Liu X, Gao Q, Li P, Zhao Q, Zhang J, Li J, Koseki H, Wong J. UHRF1 targets DNMT1 for DNA methylation through cooperative binding of hemi-methylated DNA and methylated H3K9. Nat Commun 2013, 4:1563; PMID:23463006; http://dx.doi.org/10.1038/ ncomms 2562

4. Karagianni P, Amazit L, Qin J, Wong J. ICBP90, a novel methyl $\mathrm{K} 9 \mathrm{H} 3$ binding protein linking protein ubiquitination with heterochromatin formation. Mol Cell Biol 2008; 28:705-17; PMID:17967883; http:// dx.doi.org/10.1128/MCB.01598-07

5. Esteve PO, Chin HG, Smallwood A, Feehery GR, Gangisetty O, Karpf AR, Carey MF, Pradhan S. Direct interaction between DNMT1 and G9a coordinates DNA and histone methylation during replication. Genes Dev 2006; 20:3089-103; PMID:17085482; http://dx.doi.org/10.1101/gad.1463706

6. Sharif J, Koseki H. Recruitment of Dnmt1 roles of the SRA protein Np95 (Uhrf1) and other factors. Prog Mol Biol Transl Sci 2011; 101:289-310; PMID:21507355; http://dx.doi.org/10.1016/B978-012-387685-0.00008-1

7. Kim JK, Esteve PO, Jacobsen SE, Pradhan S. UHRF1 binds G9a and participates in p21 transcriptional regulation in mammalian cells. Nucleic Acids Res 2009; 37:493-505; PMID:19056828; http://dx.doi.org/ 10.1093/nar/gkn961

8. Meilinger D, Fellinger K, Bultmann S, Rothbauer U, Bonapace IM, Klinkert WE, Spada F, Leonhardt H. Np95 interacts with de novo DNA methyltransferases, Dnmt $3 \mathrm{a}$ and Dnmt 3 b, and mediates epigenetic silencing of the viral CMV promoter in embryonic stem cells. EMBO Rep 2009; 10:1259-64; PMID:19798101; http://dx.doi.org/10.1038/embor.2009.201

9. Bronner C, Fuhrmann G, Chedin FL, Macaluso M, Dhe-Paganon S. UHRF1 Links the Histone code and DNA Methylation to ensure Faithful Epigenetic Memory Inheritance. Genet Epigenet 2010; 2009:29-36; PMID:21643543

10. Wang F, Yang YZ, Shi CZ, Zhang P, Moyer MP, Zhang HZ, Zou Y, Qin HL. UHRF1 promotes cell growth and metastasis through repression of p16(ink(4) a) in colorectal cancer. Ann Surg Oncol 2012 19:2753-62; PMID:22219067; http://dx.doi.org/ 10.1245/s10434-011-2194-1

11. Gelato KA, Tauber M, Ong MS, Winter S, HiragamiHamada K, Sindlinger J, Lemak A, Bultsma Y, Houliston S, Schwarzer D, et al. Accessibility of different histone H3-binding domains of UHRF1 is allosterically regulated by phosphatidylinositol 5-phosphate. $\mathrm{Mol}$ Cell 2014; 54:905-19; PMID:24813945; http://dx. doi.org/10.1016/j.molcel.2014.04.004

12. Bronner C, Achour M, Arima Y, Chataigneau T, Saya $\mathrm{H}$, Schini-Kerth VB. The UHRF family: oncogenes that are drugable targets for cancer therapy in the near future? Pharmacol Ther 2007; 115:419-34; PMID:17658611; http://dx.doi.org/10.1016/j. pharmthera.2007.06.003
13. Nishiyama A, Yamaguchi L, Sharif J, Johmura Y, Kawamura T, Nakanishi K, Shimamura S, Arita K, Kodama T, Ishikawa F, et al. Uhrf1-dependent H3K23 ubiquitylation couples maintenance DNA methylation and replication. Nature 2013; 502:249-53; PMID:24013172; http://dx.doi.org/10.1038/nature12488

14. Nady N, Lemak A, Walker JR, Avvakumov GV, Kareta MS, Achour M, Xue S, Duan S, Allali-Hassani A, Zuo $\mathrm{X}$, et al. Recognition of multivalent histone states associated with heterochromatin by UHRF1 protein. J Biol Chem 2011; 286:24300-11; PMID:21489993; http:// dx.doi.org/10.1074/jbc.M111.234104

15. Rothbart SB, Krajewski K, Nady N, Tempel W, Xue S, Badeaux AI, Barsyte-Lovejoy D, Martinez JY, Bedford MT, Fuchs SM, et al. Association of UHRF1 with methylated $\mathrm{H} 3 \mathrm{~K} 9$ directs the maintenance of DNA methylation. Nat Struct Mol Biol 2012; 19:1155-60; PMID:23022729; http://dx.doi.org/10.1038/nsmb.2391

16. Lallous N, Legrand P, McEwen AG, Ramon-Maiques S, Samama JP, Birck C. The PHD finger of human UHRF1 reveals a new subgroup of unmethylated histone H3 tail readers. PLoS One 2011; 6:e27599; PMID:22096602; http://dx.doi.org/10.1371/journal. pone. 0027599

17. Hu L, Li Z, Wang P, Lin Y, Xu Y. Crystal structure of PHD domain of UHRF1 and insights into recognition of unmodified histone $\mathrm{H} 3$ arginine residue 2. Cell Res 2011; 21:1374-8; PMID:21808300; http://dx.doi.org/ $10.1038 / \mathrm{cr} .2011 .124$

18. Avvakumov GV, Walker JR, Xue S, Li Y, Duan S, Bronner C, Arrowsmith CH, Dhe-Paganon S. Structural basis for recognition of hemi-methylated DNA by the SRA domain of human UHRF1. Nature 2008; 455:822-5; PMID:18772889; http://dx.doi.org/ 10.1038 /nature 07273

19. Hashimoto H, Horton JR, Zhang X, Bostick M, Jacobsen SE, Cheng X. The SRA domain of UHRF1 flips 5methylcytosine out of the DNA helix. Nature 2008; 455:826-9; PMID:18772888; http://dx.doi.org/ $10.1038 /$ nature 07280

20. Tsai WW, Wang Z, Yiu TT, Akdemir KC, Xia W, Winter S, Tsai CY, Shi X, Schwarzer D, Plunkett W, et al. TRIM24 links a non-canonical histone signature to breast cancer. Nature 2010; 468:927-32; PMID:21164480; http://dx.doi.org/10.1038/ nature 09542

21. Ruthenburg AJ, Li H, Milne TA, Dewell S, McGinty RK, Yuen M, Ueberheide B, Dou Y, Muir TW, Patel DJ, et al. Recognition of a mononucleosomal histone modification pattern by BPTF via multivalent interactions. Cell 2011; 145:692-706; PMID:21596426; http://dx.doi.org/10.1016/j.cell.2011.03.053

22. Strahl BD, Allis CD. The language of covalent histone modifications. Nature 2000; 403:41-5; PMID:10638745; http://dx.doi.org/10.1038/47412

23. Ruthenburg AJ, Li H, Patel DJ, Allis CD. Multivalent engagement of chromatin modifications by linked binding modules. Nat Rev Mol Cell Biol 2007; 8:983-94; PMID:18037899; http://dx.doi.org/10.1038/nrm2298

24. Du J, Patel DJ. Structural biology-based insights into combinatorial readout and crosstalk among epigenetic marks. Biochim Biophys Acta 2014; 1839:719-27; PMID:24747177; http://dx.doi.org/10.1016/j. bbagrm.2014.04.011

25. Cheng J, Yang Y, Fang J, Xiao J, Zhu T, Chen F, Wang P, Li Z, Yang H, Xu Y. Structural insight into coordinated recognition of trimethylated histone $\mathrm{H} 3$ lysine 9 (H3K9me3) by the plant homeodomain (PHD) and tandem tudor domain (TTD) of UHRF1 (ubiquitinlike, containing PHD and RING finger domains, 1) protein. J Biol Chem 2013; 288:1329-39; PMID:23161542; http://dx.doi.org/10.1074/jbc. M112.415398

26. Arita K, Isogai S, Oda T, Unoki M, Sugita K, Sekiyama N, Kuwata K, Hamamoto R, Tochio H, Sato M, et al. Recognition of modification status on a histone $\mathrm{H} 3$ tail by linked histone reader modules of the epigenetic regulator UHRF1. Proc Natl Acad Sci U S A 2012; 
109:12950-5; PMID:22837395; http://dx.doi.org/ 10.1073/pnas. 1203701109

27. Xie S, Jakoncic J, Qian C. UHRF1 double tudor domain and the adjacent PHD finger act together to recognize $\mathrm{K} 9 \mathrm{me} 3$-containing histone $\mathrm{H} 3$ tail. J Mol Biol 2012; 415:318-28; PMID:22100450; http://dx. doi.org/10.1016/j.jmb.2011.11.012

28. Rothbart SB, Dickson BM, Ong MS, Krajewski K, Houliston S, Kireev DB, Arrowsmith CH, Strahl BD Multivalent histone engagement by the linked tandem Tudor and PHD domains of UHRF1 is required for the epigenetic inheritance of DNA methylation. Genes Dev 2013; 27:1288-98; PMID:23752590; http://dx. doi.org/10.1101/gad.220467.113

29. Rottach A, Frauer C, Pichler G, Bonapace IM, Spada F, Leonhardt $\mathrm{H}$. The multi-domain protein $\mathrm{Np} 95$ connects DNA methylation and histone modification. Nucleic Acids Res 2010; 38:1796-804; PMID:20026581; http:// dx.doi.org/10.1093/nar/gkp1152

30. Qian C, Li S, Jakoncic J, Zeng L, Walsh MJ, Zhou MM. Structure and hemimethylated $\mathrm{CpG}$ binding of the SRA domain from human UHRF1. J Biol Chem 2008; 283:34490-4; PMID:18945682; http://dx.doi. org/10.1074/jbc.C800169200

31. Pichler G, Wolf P, Schmidt CS, Meilinger D, Schneider $\mathrm{K}$, Frauer C, Fellinger K, Rottach A, Leonhardt H. Cooperative DNA and histone binding by Uhrf2 links the two major repressive epigenetic pathways. J Cell Biochem 2011; 112:2585-93; PMID:21598301; http://dx.doi. org/10.1002/jcb.23185

32. Ma B, Tsai CJ, Haliloglu T, Nussinov R. Dynamic allostery: linkers are not merely flexible. Structure 2011; 19:907-17; PMID:21742258; http://dx.doi.org/ 10.1016/j.str.2011.06.002

33. Pendaries C, Tronchere H, Racaud-Sultan C, GaitsIacovoni F, Coronas S, Manenti S, Gratacap MP, Plantavid M, Payrastre B. Emerging roles of phosphatidylinositol monophosphates in cellular signaling and trafficking. Adv Enzyme Regul 2005; 45:201-14; PMID:16023705; http://dx.doi.org/10.1016/j. advenzreg.2005.02.006

34. Clarke JH, Letcher AJ, D'Santos CS, Halstead JR, Irvine RF, Divecha N. Inositol lipids are regulated during cell cycle progression in the nuclei of murine erythroleukaemia cells. Biochem J 2001; 357:905-10; PMID:11463365; http://dx.doi.org/10.1042/02646021:3570905

35. Balla T, Wymann M, York JD. Phosphoinositides II: The Diverse Biological Functions. Dordrecht: Springer Netherlands; 2012.

36. Barlow CA, Laishram RS, Anderson RA. Nuclear phosphoinositides: a signaling enigma wrapped in a compartmental conundrum. Trends Cell Biol 2010; 20:2535; PMID:19846310; http://dx.doi.org/10.1016/j. tcb.2009.09.009

37. Lewis AE, Sommer L, Arntzen MO, Strahm Y, Morrice NA, Divecha N, D'Santos CS. Identification of nuclear phosphatidylinositol 4,5-bisphosphate-interacting proteins by neomycin extraction. Mol Cell Proteomics 2011; 10:M110 003376; PMID:21048195; http://dx doi.org/10.1074/mcp.M110.003376

38. Trotzier MA, Bronner C, Bathami K, Mathieu E, Abbady AQ, Jeanblanc M, Muller CD, Rochette-Egly C, Mousli M. Phosphorylation of ICBP90 by protein kinase A enhances topoisomerase IIalpha expression. Biochem Biophys Res Commun 2004; 319:590-5; PMID:15178447; http://dx.doi.org/10.1016/j. bbrc.2004.05.028

39. Ma H, Chen H, Guo X, Wang Z, Sowa ME, Zheng L, Hu S, Zeng P, Guo R, Diao J, et al. M phase phosphorylation of the epigenetic regulator UHRF1 regulates its physical association with the deubiquitylase USP7 and stability. Proc Natl Acad Sci U S A 2012; 109:482833; PMID:22411829; http://dx.doi.org/10.1073/ pnas. 1116349109

40. Rigbolt KT, Prokhorova TA, Akimov V, Henningsen J, Johansen PT, Kratchmarova I, Kassem M, Mann M,
Olsen JV, Blagoev B. System-wide temporal characterization of the proteome and phosphoproteome of human embryonic stem cell differentiation. Sci Signal 2011; 4:rs3; PMID:21406692; http://dx.doi.org/ 10.1126/scisignal.2001570

41. Dephoure N, Zhou C, Villen J, Beausoleil SA, Bakalarski CE, Elledge SJ, Gygi SP. A quantitative atlas of mitotic phosphorylation. Proc Natl Acad Sci U S A 2008; 105:10762-7; PMID:18669648; http://dx.doi. org/10.1073/pnas.0805139105

42. Olsen JV, Blagoev B, Gnad F, Macek B, Kumar C, Mortensen P, Mann M. Global, in vivo, and site-specific phosphorylation dynamics in signaling networks. Cell 2006; 127:635-48; PMID:17081983; http://dx. doi.org/10.1016/j.cell.2006.09.026

43. Olsen JV, Vermeulen M, Santamaria A, Kumar C, Miller ML, Jensen LJ, Gnad F, Cox J, Jensen TS, Nigg EA, et al. Quantitative phosphoproteomics reveals widespread full phosphorylation site occupancy during mitosis. Sci Signal 2010; 3:ra3; PMID:20068231; http://dx.doi.org/10.1126/scisignal.2000475

44. Sharif J, Muto M, Takebayashi S, Suetake I, Iwamatsu A, Endo TA, Shinga J, Mizutani-Koseki Y, Toyoda T, Okamura K, et al. The SRA protein Np95 mediates epigenetic inheritance by recruiting Dnmt 1 to methylated DNA. Nature 2007; 450:908-12; PMID:17994007; http://dx. doi.org/10.1038/nature06397

45. Tsumura A, Hayakawa T, Kumaki Y, Takebayashi S, Sakaue M, Matsuoka C, Shimotohno K, Ishikawa F, L E, Ueda HR, et al. Maintenance of self-renewal ability of mouse embryonic stem cells in the absence of DNA methyltransferases Dnmt1, Dnmt3a and Dnmt3b. Genes Cell 2006; 11:805-14; PMID:16824199; http:// dx.doi.org/10.1111/j.1365-2443.2006.00984.x

46. Lehnertz B, Ueda Y, Derijck AAHA, Braunschweig U, Perez-Burgos L, Kubicek S, Chen TP, Li E, Jenuwein T, Peters AHFM. Suv39h-mediated histone H3 lysine 9 methylation directs DNA methylation to major satellite repeats at pericentric heterochromatin. Curr Biol 2003; 13:1192-200; PMID:12867029; http://dx.doi. org/10.1016/S0960-9822(03)00432-9

47. Dong KB, Maksakova IA, Mohn F, Leung D, Appanah R, Lee S, Yang HW, Lam LL, Mager DL, Schubeler D, et al. DNA methylation in ES cells requires the lysine methyltransferase G9a but not its catalytic activity. EMBO J 2008; 27:2691-701; PMID:18818693; http://dx.doi.org/10.1038/emboj.2008.193

48. Divecha N, Banfic H. The polyphosphoinositide cycle exists in the nuclei of Swiss 3T3 cells under the control of a receptor ( for IGF-1) in the plasma membrane , and stimulation of the cycle increases nuclear diacylglycerol and apparently induces translocation of protein kin. EMBO J 1991; 1:3207-14; PMID:1655412

49. Jones DR, Bultsma Y, Keune WJ, Halstead JR, Elouarrat D, Mohammed S, Heck AJ, D'Santos CS, Divecha N. Nuclear PtdIns5P as a transducer of stress signaling: an in vivo role for PIP4Kbeta. Mol Cell 2006; 23:68595; PMID:16949365; http://dx.doi.org/10.1016/j. molcel.2006.07.014

50. Jones DR, Foulger R, Keune WJ, Bultsma Y, Divecha $\mathrm{N}$. PtdIns5P is an oxidative stress-induced second messenger that regulates PKB activation. FASEB J 2013; 27:1644-56; PMID:23241309; http://dx.doi.org/ 10.1096/fj.12-218842

51. Papait R, Pistore C, Negri D, Pecoraro D, Cantarini L, Bonapace IM. Np95 is implicated in pericentromeric heterochromatin replication and in major satellite silencing. Mol Biol Cell 2007; 18:1098-106; PMID:17182844; http://dx.doi.org/10.1091/mbc. E06-09-0874

52. Achour M, Fuhrmann G, Alhosin M, Ronde P, Chataigneau T, Mousli M, Schini-Kerth VB, Bronner C. UHRF1 recruits the histone acetyltransferase Tip60 and controls its expression and activity. Biochem Biophys Res Commun 2009; 390:523-8; PMID:19800870; http://dx.doi.org/10.1016/j. bbrc.2009.09.131
53. Mistry H, Gibson L, Yun JW, Sarras H, Tamblyn L, McPherson JP. Interplay between $\mathrm{Np} 95$ and Eme1 in the DNA damage response. Biochem Biophys Res Commun 2008; 375:321-5; PMID:18692478; http://dx.doi.org/10.1016/j. bbrc.2008.07.146

54. De Vos M, El Ramy R, Quenet D, Wolf P, Spada F, Magroun N, Babbio F, Schreiber V, Leonhardt $\mathrm{H}$, Bonapace IM, et al. Poly(ADP-ribose) polymerase 1 (PARP1) associates with E3 ubiquitin-protein ligase UHRF1 and modulates UHRF1 biological functions. J Biol Chem 2014; 289:16223-38; PMID:24782312; http://dx.doi.org/10.1074/jbc. M113.527424

55. Divecha N, Banfic H, Irvine RF. The polyphosphoinositide cycle exists in the nuclei of Swiss 3T3 cells under the control of a receptor (for IGF-I) in the plasma membrane, and stimulation of the cycle increases nuclear diacylglycerol and apparently induces translocation of protein kinase $\mathrm{C}$ to the nucleus. EMBO J 1991; 10:3207-14; PMID:1655412

56. Xu Y, Xu C, Price BD. Mechanistic links between ATM and histone methylation codes during DNA repair. Prog Mol Biol Transl Sci 2012; 110:263-88; PMID:22749149; http://dx.doi.org/10.1016/B978-012-387665-2.00010-9

57. Unoki M, Nishidate T, Nakamura Y. ICBP90, an E2F1 target, recruits $\mathrm{HDAC} 1$ and binds to methyl-CpG through its SRA domain. Oncogene 2004; 23:760110; PMID:15361834; http://dx.doi.org/10.1038/sj. onc. 1208053

58. Bostick M, Kim JK, Esteve PO, Clark A, Pradhan S, Jacobsen SE. UHRF1 plays a role in maintaining DNA methylation in mammalian cells. Science 2007; 317:1760-4; PMID:17673620; http://dx.doi.org/ 10.1126/science.1147939

59. Arima Y, Hirota T, Bronner C, Mousli M, Fujiwara T, Niwa S, Ishikawa H, Saya H. Down-regulation of nuclear protein ICBP90 by p53/p21Cip1/WAF1dependent DNA-damage checkpoint signals contributes to cell cycle arrest at G1/S transition. Genes Cell 2004; 9:131-42; PMID:15009091; http://dx.doi.org/ 10.1111/j.1356-9597.2004.00710.x

60. Jeanblanc M, Mousli M, Hopfner R, Bathami K, Martinet N, Abbady AQ, Siffert JC, Mathieu E, Muller $\mathrm{CD}$, Bronner $\mathrm{C}$. The retinoblastoma gene and its product are targeted by ICBP90: a key mechanism in the G1/S transition during the cell cycle. Oncogene 2005; 24:7337-45; PMID:16007129; http://dx.doi.org/ 10.1038/sj.onc. 1208878

61. Mistry H, Tamblyn L, Butt H, Sisgoreo D, Gracias A, Larin M, Gopalakrishnan K, Hande MP, McPherson JP. UHRF1 is a genome caretaker that facilitates the DNA damage response to gamma-irradiation. Genome Integr 2010; 1:7; PMID:20678257; http://dx.doi.org/ 10.1186/2041-9414-1-7

62. Berkyurek AC, Suetake I, Arita K, Takeshita K, Nakagawa A, Shirakawa M, Tajima S. The DNA methyltransferase Dnmt1 directly interacts with the SET and RING finger-associated (SRA) domain of the multifunctional protein Uhrf1 to facilitate accession of the catalytic center to hemi-methylated DNA. J Biol Chem 2014; 289:379-86; PMID:24253042; http://dx.doi. org/10.1074/jbc.M113.523209

63. Papait R, Pistore C, Grazini U, Babbio F, Cogliati S, Pecoraro D, Brino L, Morand AL, Dechampesme AM, Spada F, et al. The PHD domain of Np95 (mUHRF1) is involved in large-scale reorganization of pericentromeric heterochromatin. Mol Biol Cell 2008; 19:355463; PMID:18508923; http://dx.doi.org/10.1091/mbc. E07-10-1059

64. Bronner C, Krifa M, Mousli M. Increasing role of UHRF1 in the reading and inheritance of the epigenetic code as well as in tumorogenesis. Biochem Pharmacol 2013; 86:1643-9; PMID:24134914; http://dx.doi.org/ 10.1016/j.bcp.2013.10.002 


\title{
Accessibility of Different Histone H3-Binding Domains of UHRF1 Is Allosterically Regulated by Phosphatidylinositol 5-Phosphate
}

\author{
Kathy A. Gelato, ${ }^{1}$ Maria Tauber, ${ }^{1}$ Michelle S. Ong, ${ }^{2}$ Stefan Winter, ${ }^{1}$ Kyoko Hiragami-Hamada, ${ }^{1}$ Julia Sindlinger, ${ }^{3}$ \\ Alexander Lemak, ${ }^{4}$ Yvette Bultsma, ${ }^{5,6}$ Scott Houliston, ${ }^{2,4}$ Dirk Schwarzer, ${ }^{3}$ Nullin Divecha, ${ }^{5,6}$ Cheryl H. Arrowsmith, ${ }^{2,4}$ \\ and Wolfgang Fischle ${ }^{1, *}$ \\ ${ }^{1}$ Laboratory of Chromatin Biochemistry, Max Planck Institute for Biophysical Chemistry, Am Fassberg 11, 37077 Göttingen, Germany \\ 2Structural Genomics Consortium, University of Toronto, 101 College Street, Toronto, ON M5G 1L7, Canada \\ 3Interfaculty Institute of Biochemistry, University of Tübingen, Hoppe-Seyler-Strasse 4, 72076 Tübingen, Germany \\ ${ }^{4}$ Princess Margaret Cancer Center, TMDT, 101 College Street, Toronto, ON M5G 1L7, Canada \\ ${ }^{5}$ Cancer Research UK Manchester Institute, The University of Manchester, Wilmslow Road, Manchester M20 4BX, UK \\ ${ }^{6}$ Present address: Centre for Biological Sciences, University of Southampton, Life Sciences Building 85, Highfield, \\ Southampton SO17 1BJ, UK \\ *Correspondence: wfischl@gwdg.de \\ http://dx.doi.org/10.1016/j.molcel.2014.04.004
}

\section{SUMMARY}

UHRF1 is a multidomain protein crucially linking histone $\mathrm{H} 3$ modification states and DNA methylation. While the interaction properties of its specific domains are well characterized, little is known about the regulation of these functionalities. We show that UHRF1 exists in distinct active states, binding either unmodified $\mathrm{H} 3$ or the $\mathrm{H} 3$ lysine 9 trimethylation (H3K9me3) modification. A polybasic region (PBR) in the $\mathrm{C}$ terminus blocks interaction of a tandem tudor domain (TTD) with $\mathrm{H} 3 \mathrm{~K} 9 \mathrm{me} 3$ by occupying an essential peptide-binding groove. In this state the plant homeodomain (PHD) mediates interaction with the extreme $\mathrm{N}$ terminus of the unmodified $\mathrm{H} 3$ tail. Binding of the phosphatidylinositol phosphate PI5P to the PBR of UHRF1 results in a conformational rearrangement of the domains, allowing the TTD to bind H3K9me3. Our results define an allosteric mechanism controlling heterochromatin association of an essential regulatory protein of epigenetic states and identify a functional role for enigmatic nuclear phosphatidylinositol phosphates.

\section{INTRODUCTION}

A major concept that has emerged for the readout of histone modifications is the recruitment of proteins containing conserved domains that specifically interact with defined modifications. Systematic proteomic approaches have implied that individual chromatin modifications are usually recognized by multiple competing proteins (Patel and Wang, 2013). To understand the readout of chromatin marks, it is therefore imperative to dissect how the interplay between modifications and binding factors is regulated. The exact context appears to matter, as neighboring modifications can influence interaction in a negative or positive manner. Posttranslational modifications of chromatin-associated proteins can directly affect the modification binding properties of chromatin proteins. Further, the expression of specific binding factors is modulated in cell differentiation and development (reviewed by Fischle, 2012).

Since transient changes in cellular states (e.g., nutrition levels) can have a lasting effect on gene expression patterns, it has been suggested that metabolites and small cellular molecules have an immediate impact on the condition of chromatin and the epigenetic state of cells (Lu and Thompson, 2012). Phosphatidylinositol phosphates (PIPs) are particularly interesting in the context of chromatin. A pool of these lipids exists in the cell nucleus, separately regulated from the cell membrane and cytoplasm by dedicated enzymes. However, the exact biochemical state of PIPs in this compartment is still unclear (Barlow et al., 2010; Fiume et al., 2012). The bulk of nuclear phospholipids copurifies with nonhistone chromosomal proteins (Manzoli et al., 1976). Interestingly, analysis of isolated chromatin fractions revealed that lipids associated with hetero- and euchromatin each showed distinct turnover rates (Rose and Frenster, 1965). Furthermore, hydrolysis of nuclear phospholipids by phospholipase $\mathrm{C}$ (PLC) was shown to change chromatin structure (Maraldi et al., 1984).

Few nuclear proteins have been found to associate with PIPs so far (Barlow et al., 2010; Fiume et al., 2012). PI4,5P 2 has been shown to activate and stabilize the chromatin remodeling complex BAF (Burgio et al., 2010). The H3K4me3-binding plant homeodomain (PHD) and adjacent polybasic region of ING2 and several related proteins associate with chromatin through a PI5P-mediated mechanism (Bua and Binda, 2009; Gozani et al., 2003; Jones et al., 2006). While this pathway is important in controlling ING2-dependent genes, especially in DNA damage pathways, interaction of $\mathrm{H} 3 \mathrm{~K} 4 \mathrm{me} 3$ and $\mathrm{PI5P}$ are independent of each other (Bua et al., 2013). Binding of PI5P to a PHD domain causes ATX1 to detach from promoters and translocate from the nucleus to the cytosol (Ndamukong et al., 2010). Whereas major biochemical screens have been conducted for PIP-binding factors in the cytoplasmic fraction (Catimel et al., 2009; 
A

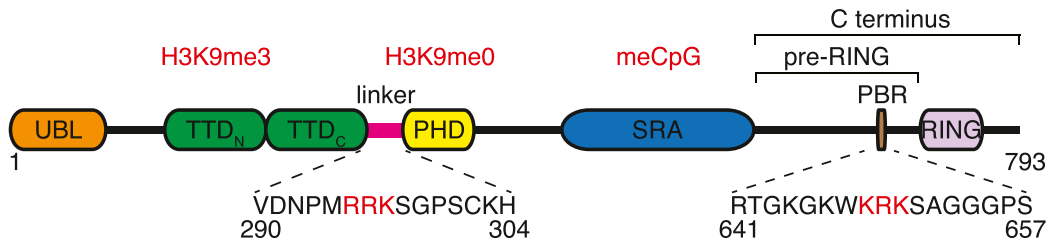

B

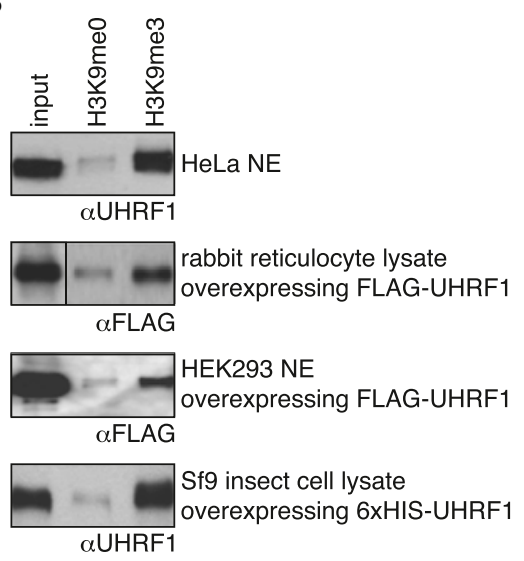

D

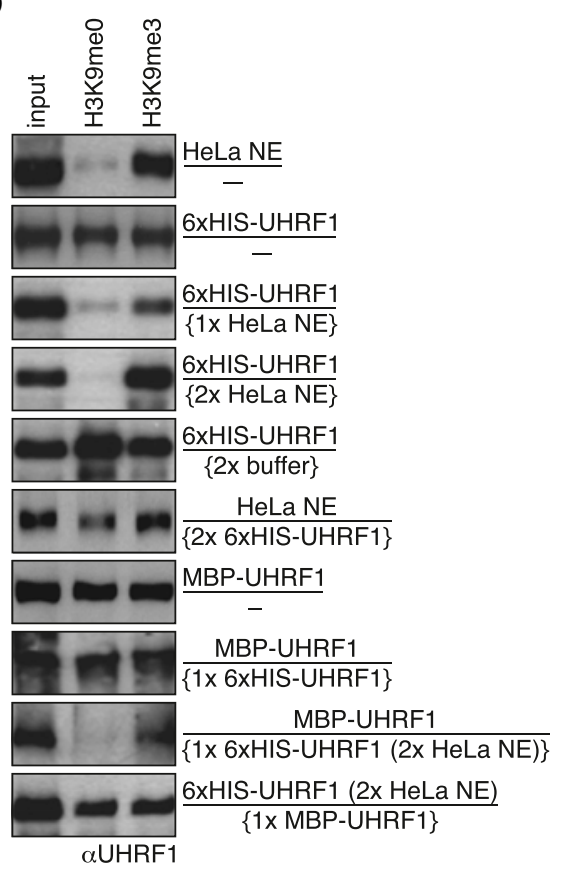

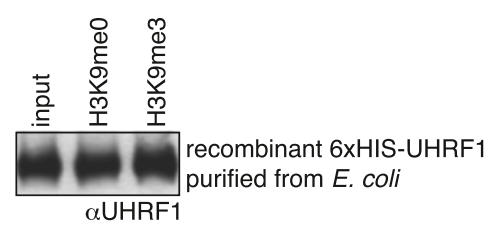
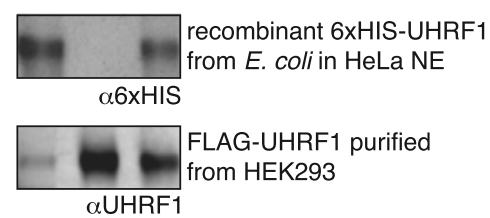

$=6 x$ HIS-UHRF1 purified 0.5 from Sf9 insect cells $\alpha \mathrm{UHRF} 1$

E

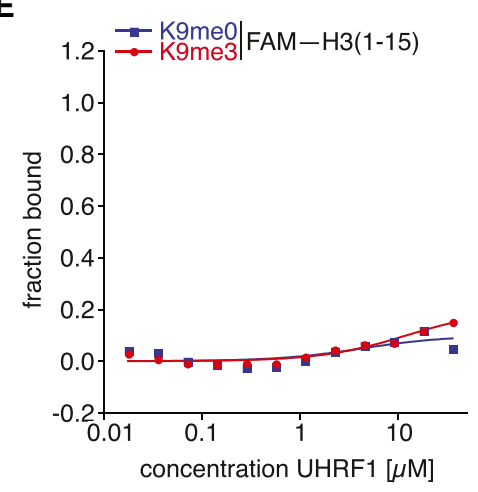

G

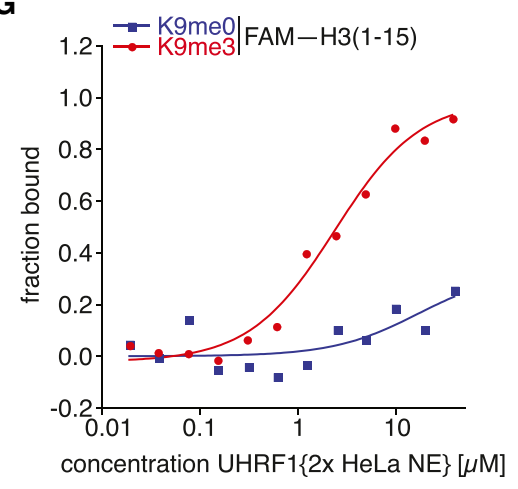

C
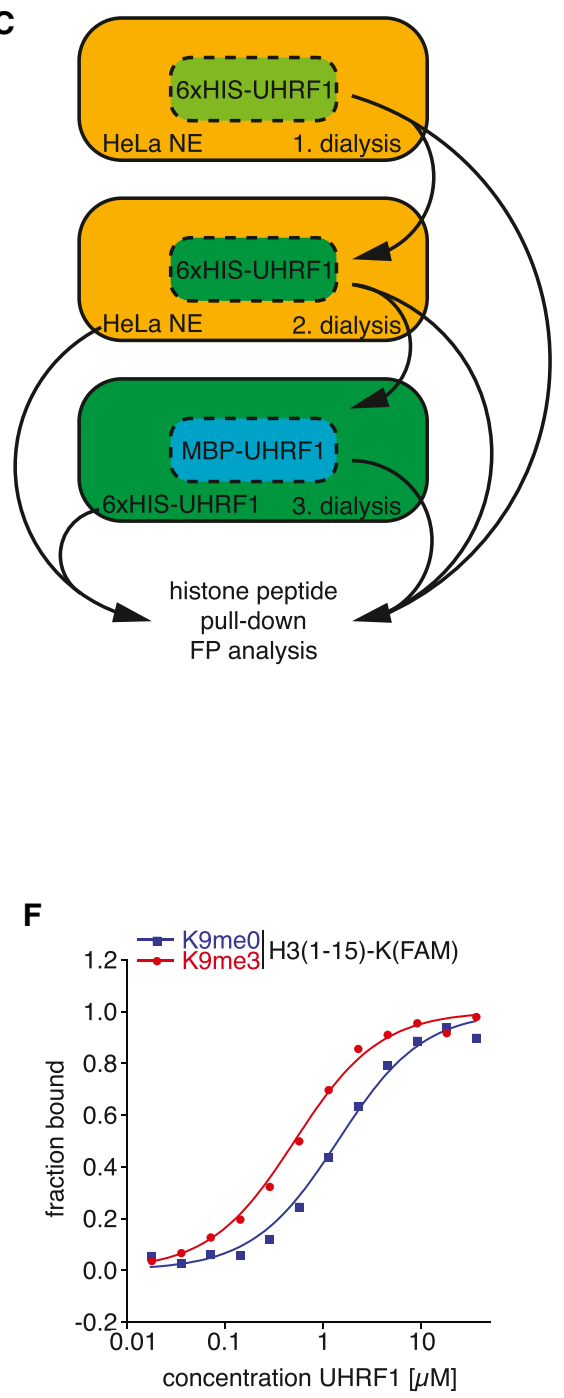

H

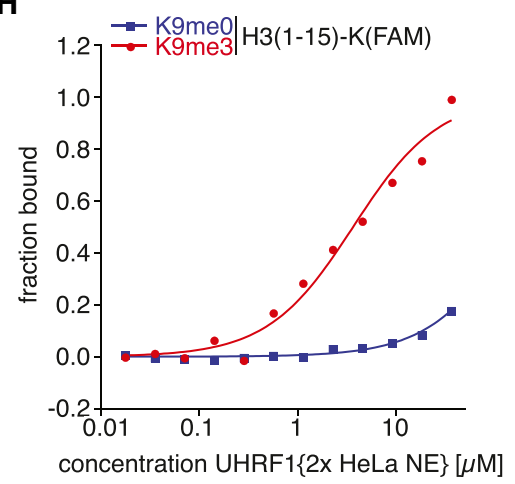

Figure 1. UHRF1 Binding to the Modified or Unmodified H3 Tail Is Regulated by Cellular Cofactors

(A) Domain structure of UHRF1. UBL, ubiquitin-like (aa 1-76); TTD, tandem tudor domain (aa 126-285); PHD, plant and homeodomain (aa 310-366); SRA, SETand RING-associated (aa 435-586); PBR, polybasic region; RING, really interesting new gene (aa 724-763).

(B) Cellular lysates (NE, nuclear extract) and purified cellular or recombinant proteins were incubated with the specified biotinylated H3(1-20) peptides immobilized on streptavidin magnetic beads. Recovered material was analyzed by western blot. Input, $2 \%$.

(C) Flow scheme for dialysis experiments as analyzed in (D)-(H). 
Pasquali et al., 2007), only one study attempted to define the nuclear interactome of a PIP, namely PI4,5P 2 . There, lysine/arginine-rich patches with the consensus $K / R-(X)_{n=3-7}-K-X-K / R-K /$ $R$ emerged as major interaction motifs (Lewis et al., 2011).

Ubiquitin-like with PHD and RING finger domains 1 (UHRF1), also known as inverted CCAAT box protein of $90 \mathrm{kDa}$ (ICBP90) and NP95 in mouse, is a nuclear multidomain factor implicated in the maintenance of DNA methylation patterns during replication. Deletion of UHRF1 in mice is embryonic lethal. The knockout embryonic stem cells show loss of DNA methylation, enhanced susceptibility to DNA replication arrest, increased sensitivity to DNA damaging agents, impaired maintenance of higher-order chromatin structure, and spurious transcription of repetitive DNA elements (Bostick et al., 2007; Sharif et al., 2007). UHRF1 is upregulated in various cancer cells, including breast, prostate, and lung cancer, where it plays a key role in promoting proliferation (Bronner et al., 2010).

UHRF1 is composed of at least five domains: an N-terminal ubiquitin-like domain (UBL), followed by a tandem tudor domain (TTD), a plant homeodomain (PHD), a SET and RING-associated (SRA) domain, and a C-terminal really interesting new gene (RING) domain (Figure 1A). The SRA domain preferentially binds to hemimethylated $\mathrm{CpG}$, which during semiconservative replication of DNA recruits DNMT1 to copy the methylation pattern onto the daughter strand (Berkyurek et al., 2014; Rottach et al., 2010; Sharif and Koseki, 2011). This process appears to also involve H3K23 ubiquitylation by the RING (Nishiyama et al., 2013). The isolated TTD binds H3K9me3 (Nady et al., 2011), while the isolated $\mathrm{PHD}$ recognizes the unmodified extreme $\mathrm{H} 3 \mathrm{~N}$ terminus (Rajakumara et al., 2011). Functional cooperation by these modules was recently suggested by structural and functional studies on isolated TTD-PHD fragments (Arita et al., 2012; Cheng et al., 2013; Rothbart et al., 2013). Both DNA methylation and $\mathrm{H} 3 \mathrm{~K} 9 \mathrm{me} 3$ are hallmarks of pericentric heterochromatin where UHRF1 is preferentially localized (Liu et al., 2013). UHRF1 is also found in euchromatin and regulates gene expression, particularly the silencing of tumor suppressor genes, possibly through affecting DNA methylation and histone modifications (Bronner et al., 2010; Wang et al., 2012). Posttranslational modification of UHRF1 has been implicated in regulating its stability and possibly directing its interaction with chromatin components (Arita et al., 2012; Ma et al., 2012).

While the interactions of many histone modification-binding domains with their target sites are well studied, it is not always clear if and how the findings on the isolated modules pertain to the proteins and complexes containing them. Here, we analyzed the histone modification binding properties of intact, full-length UHRF1. We found that the protein is allosterically regulated for interaction with unmodified $\mathrm{H} 3$ versus $\mathrm{H} 3 \mathrm{~K} 9$ me3, principally by PI5P, which controls access to the TTD and PHD domains. Our results imply that this mechanism contributes to directing UHRF1 heterochromatin localization and function.

\section{RESULTS}

\section{UHRF1 Exists in Different Functional States}

To obtain insights into the recognition of histone marks by UHRF1, we performed histone peptide pull-down experiments using the lysate or purified form of the FLAG- or $6 \times \mathrm{HIS}$ tagged protein from different expression systems. Endogenous UHRF1 in HeLa cell nuclear extract (NE) exhibited preference for H3K9me3 over the unmodified form (H3K9me0) (Figure 1B). Similar binding specificity was observed for UHRF1-FLAG in vitro translated in rabbit reticulocyte extract or in NE prepared from HEK293 cells overexpressing the fusion protein and for $6 \times$ HIS-UHRF1 in total lysate of Sf9 insect cells programmed for expression. In contrast, recombinant $6 \times$ HIS-UHRF1 from $E$. coli bound the $\mathrm{H} 3 \mathrm{~K} 9 \mathrm{meO}$ and $\mathrm{H} 3 \mathrm{~K} 9 \mathrm{me} 3$ states equally well. Similarly, the UHRF1-FLAG or $6 \times$ HIS-UHRF1 proteins affinity purified from the overexpressing human embryonic kidney 293 (HEK293) or Sf9 cell extracts, respectively, displayed comparable interaction with both $\mathrm{H} 3$ tail peptides. Bacterial, recombinant $6 \times$ HIS-UHRF1 preferentially bound H3K9me3 when put into HeLa NE. As we observed distinct behavior in multiple expression systems and with different affinity tags at the $\mathrm{N}$ and $\mathrm{C}$ terminus, we concluded that the proteins in the NE and those that are affinity purified likely exist in different functional states.

\section{UHRF1 Interaction with the Modified or Unmodified H3 Tail Is Regulated by Cellular Cofactors}

Similar to previous findings (Nady et al., 2011), we observed that the isolated TTD of UHRF1 specifically binds to H3K9me3 (Figure $\mathrm{S} 1 \mathrm{~A}$ available online). In contrast, the PHD domain interacted with the extreme $\mathrm{H} 3$ tail irrespective of the modification status of the K9 site (Hu et al., 2011; Lallous et al., 2011; Rajakumara et al., 2011; Wang et al., 2011). This behavior was reflected in quantitative fluorescence polarization binding experiments (FP). We used two sets of peptides corresponding to residues 1-15 of the $\mathrm{H} 3$ tail with fluorescein attached to either the $\mathrm{N}$ or $\mathrm{C}$ terminus. Both types of peptides carrying the K9me3 mark bound to the TTD with similar affinity, while no interaction with the unmodified counterpart was observed (Figures S1B and S1C; see Tables S1 and $\mathrm{S} 2$ for a listing of all $\mathrm{K}_{\mathrm{D}}$ values measured in this study). In contrast, the PHD only bound the C-terminally labeled peptides irrespective of the modification status of $\mathrm{K} 9$, but not the

(D) Cellular lysates or recombinant proteins (top) were dialyzed against the indicated sources (bottom) and analyzed by histone peptide pull-down as in (B).

(E) Fluorescence polarization binding experiment using purified recombinant $6 \times$ HIS-UHRF1 directly. H3 peptides were linked to fluorescein at the $\mathrm{N}$ terminus (FAM-H3[1-15]) via an amide bond.

(F) Fluorescence polarization binding experiment using purified recombinant $6 \times \mathrm{HIS}-\mathrm{UHRF} 1$ directly. A non-natural lysine at the $\mathrm{C}$ terminus was added and labeled with fluorescein at the $\varepsilon$-amino group (H3[1-15]-K[FAM]).

(G) Fluorescence polarization binding experiment using purified recombinant $6 \times$ HIS-UHRF1 after dialysis against HeLa NE. H3 peptides were linked to fluorescein at the $\mathrm{N}$ terminus (FAM-H3[1-15]) via an amide bond.

(H) Fluorescence polarization binding experiment using purified recombinant $6 \times \mathrm{HIS}-\mathrm{UHRF} 1$ after dialysis against HeLa NE. A non-natural lysine at the $\mathrm{C}$ terminus was added and labeled with fluorescein at the $\varepsilon$-amino group (H3[1-15]-K[FAM]). See also Figure S1 and Tables S1 and S2. 
$\mathrm{N}$-terminally labeled forms. Our findings provided an experimental approach to quantitatively define the interaction properties of UHRF1 and to dissect the contributions of the TTD and PHD within the full-length protein.

Since the observed $\mathrm{H} 3$ methylation-specific interaction of UHRF1 in the cell extract resembled that of the TTD, while the methylation nonspecific interaction of purified UHRF1 corresponded to that of the PHD, we reasoned that cellular cofactors direct differential binding modes relying on one or the other module. To test this, we set up a dialysis experiment, consecutively exposing recombinant $6 \times$ HIS-UHRF1 purified from E.coli to HeLa NE, but separated by a membrane with a very low (3 kDa) molecular weight cutoff (Figure 1C). After one round of dialysis, interaction with $\mathrm{H} 3 \mathrm{~K} 9 \mathrm{meO}$ was significantly decreased, while binding to H3K9me3 appeared unaffected (Figure 1D). After two rounds of dialysis, the interaction of the recombinant, dialyzed protein fully resembled that of the endogenous UHRF1 in HeLa NE. In parallel, the endogenous UHRF1 protein in the HeLa NE used for dialysis changed its properties, now binding $\mathrm{H} 3 \mathrm{~K} 9 \mathrm{meO}$ and $\mathrm{H} 3 \mathrm{~K} 9 \mathrm{me} 3$ peptides equally well. Similar behavior was observed when NE from Sf9 insect cells instead of HeLa NE was used, indicating a general phenomenon (Figure S1D).

Quantitative FP measurements confirmed this switch in binding behavior. Purified, recombinant $6 \times$ HIS-UHRF1 did not bind $\mathrm{N}$-terminally labeled $\mathrm{H} 3 \mathrm{~K} 9 \mathrm{meO}$ or $\mathrm{H} 3 \mathrm{~K} 9 \mathrm{me} 3$ peptides (Figure 1E). Conversely, the protein interacted with C-terminally labeled $\mathrm{H} 3$ peptides with a slight preference for the K9me3 over the $\mathrm{K} 9 \mathrm{meO}$ state (Figure 1F). After two rounds of dialysis against HeLa NE, the properties changed completely. Interaction with $\mathrm{H} 3 \mathrm{~K} 9 \mathrm{me} 0$ peptides irrespective of labeling at the $\mathrm{N}$ or $\mathrm{C}$ terminus was lost, while the H3K9me3 mark on both substrates was recognized, indicating that the TTD was responsible for the interaction and that any PHD binding contribution was removed (Figures $1 \mathrm{G}$ and $1 \mathrm{H}$ ). We then performed consecutive dialysis experiments using UHRF1 with different tags (Figure 1D). Recombinant, bacterially expressed MBP-UHRF1, which bound equally well to $\mathrm{H} 3 \mathrm{~K} 9 \mathrm{meO}$ and $\mathrm{H} 3 \mathrm{~K} 9 \mathrm{me} 3$ peptides, was dialyzed against $6 \times$ HIS-UHRF1 that had been already dialyzed twice against HeLa NE. Pull-down experiments showed that MBP-UHRF1 could be activated for specific H3K9me3 interaction using this scheme. Overall, the results indicated that small cofactors are transferred from HeLa NE to a recombinant protein, which can also be transferred to another recombinant protein.

\section{Different H3 Tail Binding Modes of UHRF1 Are Mediated by the TTD and PHD Domains}

We reasoned that the differential behavior of UHRF1 might be the consequence of allosteric regulation inducing conformational changes of the protein. Therefore, we compared the hydrodynamic properties of purified recombinant UHRF1 before and after dialysis against HeLa NE by analytical ultracentrifugation (Figure 2A). UHRF1 in a matched buffer control had a sedimentation coefficient around $3.7 \mathrm{~S}$. The fit in the continuous $\mathrm{c}(\mathrm{s}, \mathrm{ffO})$ model suggested that the majority of molecules displayed a frictional coefficient around 1.66 (Schuck and Rossmanith, 2000). After dialysis against HeLa NE, this distribution was significantly shifted toward lower frictional coefficients, demonstrating that the protein adopted a different overall conformation.
To gain further insights into the regulation of UHRF1, we analyzed a series of mutant recombinant proteins (Figure 2B). Deletion of the TTD had no effect on the interaction of UHRF1 with $\mathrm{H} 3 \mathrm{~K} 9 \mathrm{meO}$ and $\mathrm{H} 3 \mathrm{~K} 9 \mathrm{me} 3$. Deletion of the PHD or mutagenesis of two key residues therein abolished $\mathrm{H} 3$ tail binding in pulldown (Figure 2C) and FP experiments (Figures S2A and S2B). The same mutations, however, had no effect on the interaction of the protein dialyzed against HeLa NE with H3K9me3. The results were consistent with the PHD mediating H3K9me0/ $\mathrm{H} 3 \mathrm{~K} 9 \mathrm{me} 3$ interaction in the form of the recombinant protein and the TTD mediating $\mathrm{H} 3 \mathrm{~K} 9 \mathrm{me} 3$ binding after dialysis against HeLa NE. They also implied that the TTD is unavailable for histone binding in the state of the pure recombinant protein.

Deletion of the C-terminal region resulted in a recombinant UHRF1 protein that specifically recognized the H3K9me3 peptide in pull-down (Figure 2B) as well as FP (Figure 2D) experiments, reminiscent of the wild-type recombinant protein after HeLa NE dialysis. In this construct, the TTD is clearly available for binding the $\mathrm{H} 3 \mathrm{~K} 9 \mathrm{me} 3$ tail. While a fragment containing only the TTD, PHD, and SRA domains showed similar preference for $\mathrm{H} 3 \mathrm{~K} 9 \mathrm{me} 3$, the isolated TTD-PHD fragment displayed intermediate behavior. It bound to $\mathrm{H} 3 \mathrm{~K} 9 \mathrm{me} 3$ and, somewhat weaker, to $\mathrm{H} 3 \mathrm{~K} 9 \mathrm{me}$. FP analysis indicated that this fragment could interact with the C-terminally labeled H3K9meO and H3K9me3 peptides and also with the N-terminally labeled H3K9me3 peptide, but not with the $\mathrm{N}$-terminally labeled $\mathrm{H} 3 \mathrm{~K} 9$ meO peptide (Figure S2C). Binding to the C-terminally labeled peptides was overall stronger compared to the $\mathrm{N}$-terminally labeled peptide, and there was a slight preference for the H3K9me3 over the H3K9me0 state. Recent work has suggested a cooperative mode of interaction involving simultaneous recognition of the extreme $\mathrm{N}$ terminus of $\mathrm{H} 3$ by the PHD and of the K9me3 mark by the TTD in this context (Arita et al., 2012; Cheng et al., 2013; Rothbart et al., 2013; Xie et al., 2012). However, since the full-length protein dialyzed against HeLa NE did not bind the C-terminally labeled $\mathrm{H} 3 \mathrm{~K} 9 \mathrm{me} 0$ peptide (Figure $1 \mathrm{H}$ ), meaning that the PHD is not contributing to $\mathrm{H} 3$ interaction, but showed binding to the N-terminally labeled H3K9me3 peptide similar to the TTD (Figure S2D), we deduced that the behavior of the isolated TTD-PHD could not explain the binding properties of the full-length protein.

\section{A PBR Sequence in the C-Terminal Domain of UHRF1 Binds to a Peptide-Binding Groove on the Surface of the TTD}

Because of the altered methylation specificity of the UHRF1 C-terminal deleted protein, we further investigated the role of this region in regulating $\mathrm{H} 3$ tail binding. In immunoprecipitation experiments, the isolated $\mathrm{C}$ terminus bound to the isolated TTD (Figure S3A), but not the PHD domains of UHRF1 (Figure S3B) or the FYVE domain of the Eea1 protein (Figure S3C), which belongs to the RING superfamily. Isothermal titration calorimetry deduced a binding strength of the isolated $\mathrm{C}$ terminus to the TTD of $4 \mu \mathrm{M}$ (Figure S3D).

Additional mapping identified a short region within the preRING region of the $C$ terminus that was sufficient for binding the TTD in pull-down experiments (Figure 3A). Due to its high content of basic amino acids, we termed this stretch a polybasic 
A
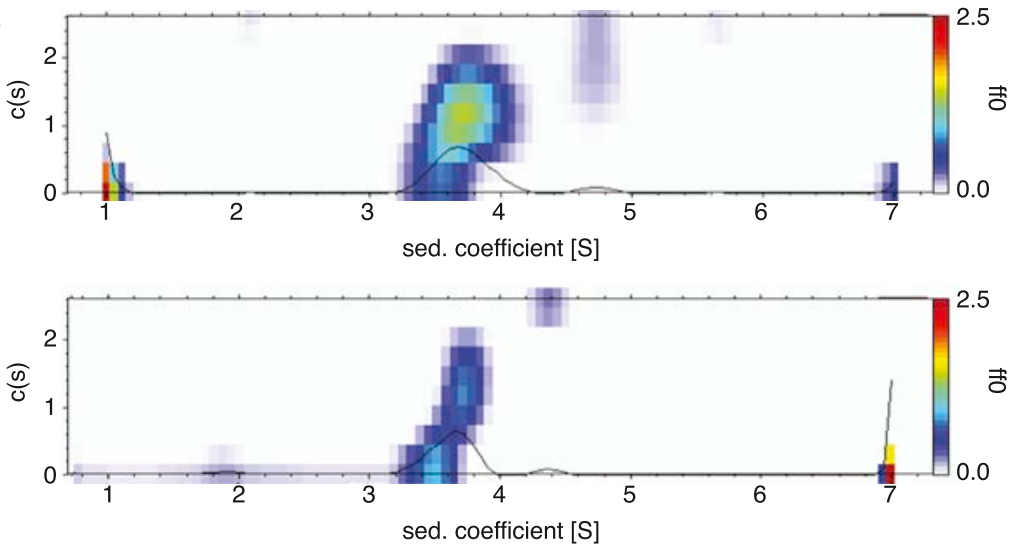

B

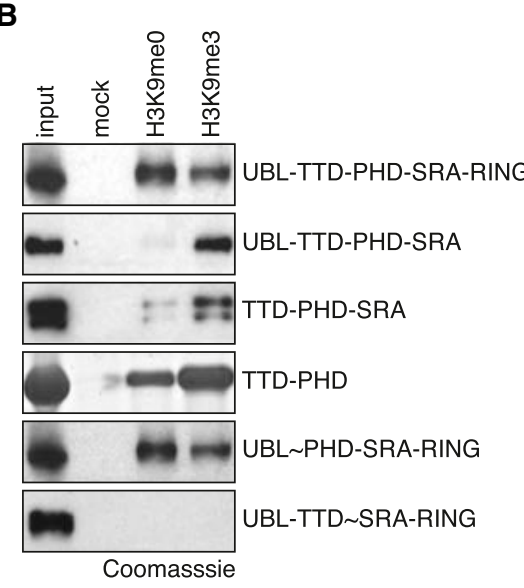

C

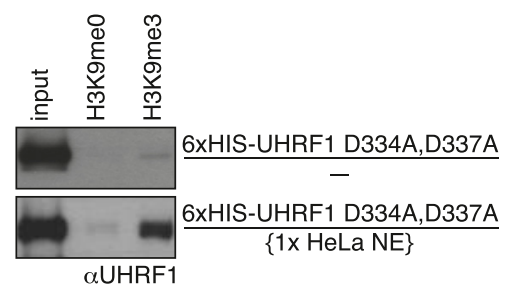

D

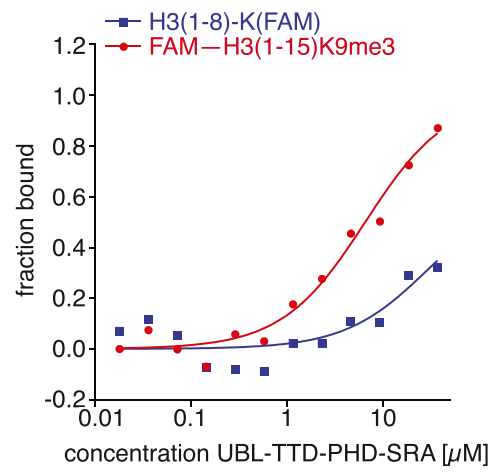

Figure 2. The C Terminus Blocks UHRF1 H3K9me3 Interaction

(A) Recombinant $6 \times$ HIS-UHRF1 either dialyzed against buffer (top) or against HeLa NE (bottom) was analyzed by analytical ultracentrifugation. Distribution of sedimentation coefficient ( $\mathrm{S}, \mathrm{x}$ axis), concentration (c[S]; left y axis), and frictional ratio (f/f0; right $y$ axis; intensity of the corresponding sedimentation coefficient is indicated by superposition of the heatmap).

(B) Recombinant proteins were incubated with the specified biotinylated $\mathrm{H} 3$ (1-20) peptides immobilized on streptavidin magnetic beads. Recovered material was analyzed by western blot. Input, 2\%.

(C) Mutant recombinant UHRF1 protein was either analyzed directly or after dialysis against HeLa NE by histone pull-down experiment as in (B).

(D) Recombinant UHRF1 lacking the $\mathrm{C}$ terminus was analyzed in fluorescence polarization binding experiments. See also Figure S2 and Tables S1 and S2.

shift changes larger than 0.094 ppm were concentrated around the groove formed at the interface between the two tudor domains (Figure S3F). This region was previously shown to bind the sequence $\mathrm{N}$-terminal to the methylated lysine in the complex of the TTD with H3K9me3 (Nady et al., 2011). It also accommodates the linker in the crystal structure of the H3K9me3/TTD-PHD complex, thereby directing the cooperative binding mode engaging both modules (Arita et al., 2012; Cheng et al., 2013; Rothbart et al., 2013; Xie et al., 2012). We therefore refer to this region of the TTD as the peptide-binding groove (Figure 3D). Although the PBR can bind to the same TTD peptide-binding groove as region (PBR) (Figure 1A). Previous analyses of the cooperative binding mode of the UHRF1 TTD-PHD fragment have implicated the short linker region between these domains in directing the synergistic recognition of H3K9me3 (Arita et al., 2012; Cheng et al., 2013; Rothbart et al., 2013; Xie et al., 2012). A peptide binding groove on the isolated TTD was found to bind to $\mathrm{H} 3$ tail peptides with low $\mu \mathrm{M}$ affinity (Nady et al., 2011) as well as to the TTD-PHD linker with relatively weak affinity $\left(K_{D}>\right.$ $100 \mu \mathrm{M})$. Interestingly, the PBR, the TTD-PHD linker region, and the $\mathrm{H} 3$ tail all share an RKS sequence motif (Figure $3 \mathrm{~B}$ ). We measured the $K_{D}$ for binding of the TTD to the PBR at $4 \mu \mathrm{M}$, which was much lower than that for the free TTD-PHD linker peptide (Figure 3C) (Rothbart et al., 2013).

In order to further characterize the intramolecular interaction, we performed a nuclear magnetic resonance (NMR) titration of increasing concentrations of the PBR peptide into ${ }^{15} \mathrm{~N}$-labeled TTD. We observed significant chemical shift changes indicative of an interaction in the micromolar range (Figure S3E). Chemical the histone $\mathrm{H} 3$ peptide and the TTD-PHD linker, the observed chemical shift perturbations were more extensive, involving extra surface residues such as K187, E193, L225, I211, and D275 (Figures 3D and S3F).

To better understand how the PBR interacts with the TTD, we performed docking calculations with HADDOCK, which uses NMR chemical shift changes to guide the simulations (de Vries et al., 2007; Dominguez et al., 2003). Two plausible models of the docked PBR were deduced. These differed in the direction of the bound PBR peptide (Figure 3E). Model 1 suggested an important contribution of PBR residues R649 and K650, while model 2 implicated residues K648 and R649 for binding to the TTD. Mutagenesis analysis of PBR peptides by FP indicated an essential contribution of R649 (Figure 3F). Mutagenesis of K648 had a stronger effect compared to mutagenesis of K650. In contrast, mutagenesis of residues $\mathrm{K} 644$ and K646 had no effect on the interaction. Similar results were obtained for the TTD-PHD fragment (Figure S3G). While we were not able to 
A

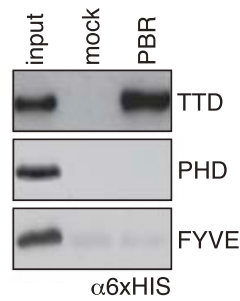

E

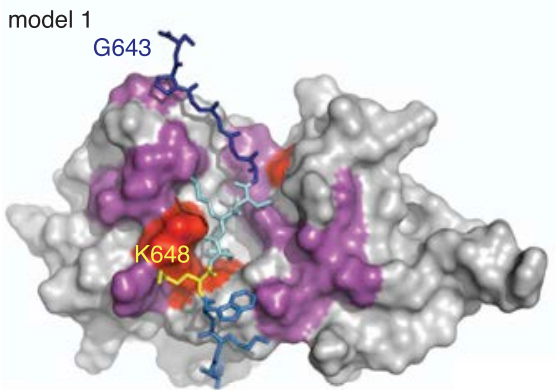

G

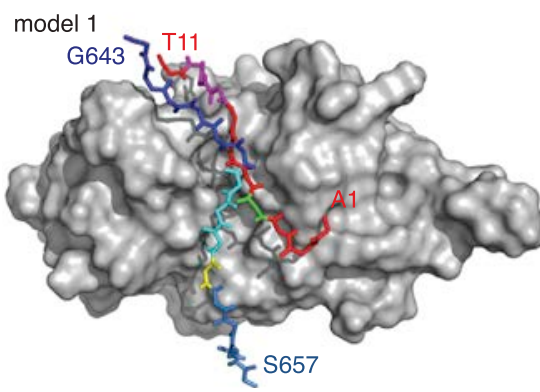

B
PBR 643--GKGKWKRKSAGGGPS -657

linker 290--VDNPMRRKSGPSCKH-304

H3 1-ARTKQTARKSTGGKAP-16

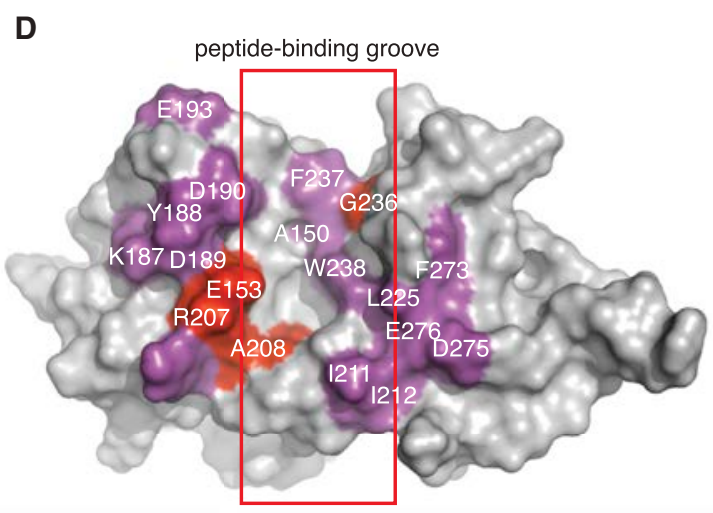

model 2

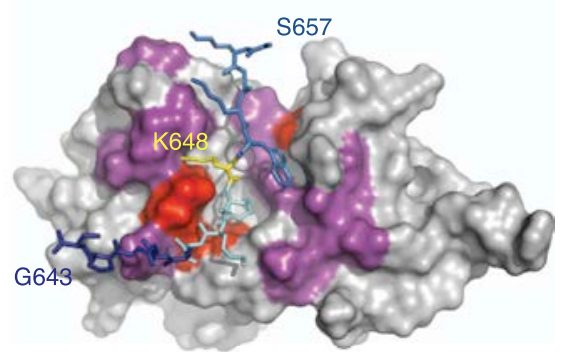

model 2

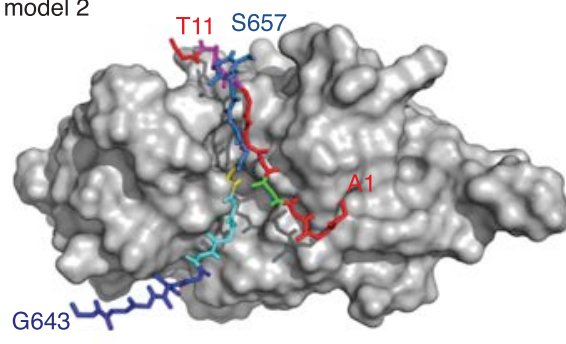

C

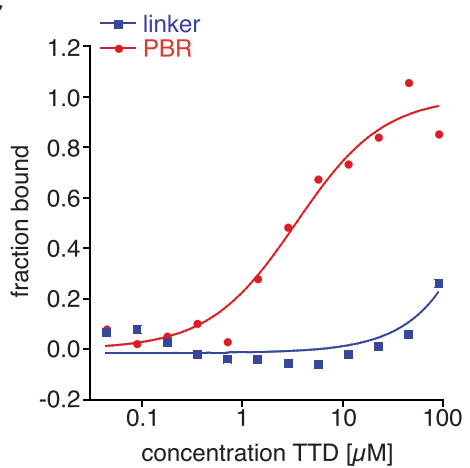

$\mathbf{F}$

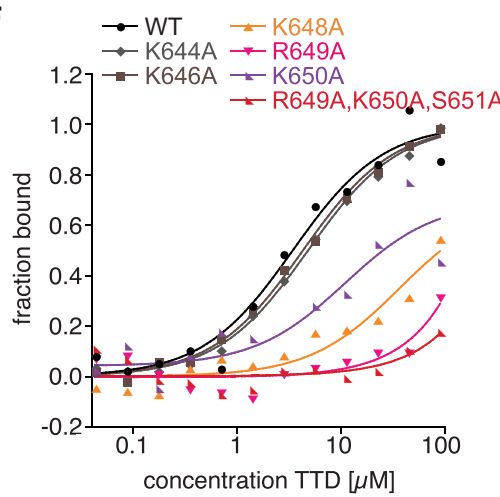

H

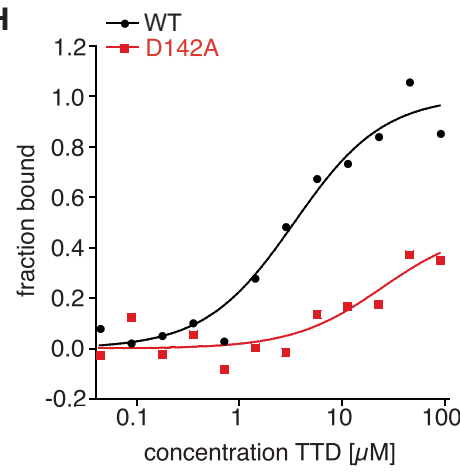

Figure 3. The PBR Sequence of the Pre-RING Region Binds a Peptide Groove on the Surface of the TTD

(A) TTD or PHD domains of UHRF1 or the FYVE domain of Eea1 were incubated with a biotinylated PBR peptide immobilized on streptavidin magnetic beads or beads alone (mock). Recovered material was analyzed by western blot. Input, $2 \%$.

(B) Sequence comparison of the UHRF1 PBR and linker regions with the H3 tail.

(C) TTD of UHRF1 was analyzed in fluorescence polarization binding experiments with peptides corresponding to the UHRF1 linker or PBR (FAM at the $\mathrm{N}$ terminus).

(D) Surface model of the TTD (Protein Data Bank ID [PDB] 2L3R). Residues with chemical shift changes $>0.094$ ppm upon titration of the PBR peptide are shown in purple; those lining the TTD peptide-binding groove (red box), which have disappeared upon titration of the PBR peptide, are shown in red.

(E) HADDOCK models of likely positions of the PBR peptide in the TTD peptide-binding groove. K648 is highlighted in yellow as a visual guide of the orientation of the peptide; R649, K650, S651 is shown in cyan; the $\mathrm{N}$ and $\mathrm{C}$ termini of the peptide are shown in blue and dark blue, respectively. Color codes of residues showing major changes upon addition of the PBR peptide are as in (D).

(F) TTD of UHRF1 was analyzed in fluorescence polarization binding experiments with the indicated wild-type (WT) or mutant FAM-PBR peptides.

(G) Overlay of the positioning of the $\mathrm{H} 3$ tail (red; $\mathrm{K} 4$ residue in green, $\mathrm{K} 9$ residue in purple) as determined in the TTD/H3K9me3 complex (PDB 2L3R) and the PBR sequence in model 1 and model 2 (color code as in E). Images were generated by PyMOL.

(H) Wild-type (WT) or D142A mutant TTD of UHRF1 was analyzed in fluorescence polarization binding experiments with WT FAM-PBR peptide. See also Figure S3 and Tables S1 and S2. 
unambiguously discriminate between the two binding modes for the TTD/PBR interaction, the mutagenesis results favored model 2. Comparison of the TTD/PBR complex with the TTD/H3K9me3 (Figure 3G) or the TTD/linker (Figure S3H) complexes indicated a mutually exclusive binding mode.

While methylated $\mathrm{K} 9$ of $\mathrm{H} 3$ within the RKS motif binds in a pocket formed in the TTD, the RKS sequence of the PBR does not interact in the same region. Instead, the UHRF1 PBR is centrally located in the TTD peptide-binding groove. Indeed, mutagenesis of two caging aromatic acid residues implicated in methyl-lysine binding in the TTD/H3K9me3 complex had only a very limited effect on TTD/PBR interaction (Figure S3I). In both NMR models, R649 engages in hydrogen bonding with $\mathrm{D} 142$, and mutagenesis of this residue significantly impaired binding of the PBR to the TTD (Figure $3 \mathrm{H}$ ). In model 2, K648 engages in hydrogen bonding with D189 and D190. These are critical residues in the TTD peptide-binding groove that interact with R296 of the anchored TTD-PHD linker and with T6 of the histone H3K9me3 peptide, respectively (Arita et al., 2012; Nady et al., 2011). While mutagenesis of R295 and R296 of the linker within the TTD-PHD fragment had no effect on PBR interaction, it resulted in a slight increase in binding to free linker peptide (Figure S3J).

\section{The PBR Sequence Is Sufficient for Blocking H3K9me3 Binding of the TTD}

Next, we investigated the role of the C-terminal domain of UHRF1 in regulating H3K9me3 binding of the TTD. While the $\mathrm{C}$ terminus did not show specific interaction with $\mathrm{H} 3 \mathrm{~K} 9 \mathrm{meO}$ or H3K9me3 peptides (Figures S4A and S4B), it severely reduced recovery of the TTD on $\mathrm{H} 3 \mathrm{~K} 9 \mathrm{me} 3$ beads when titrated into histone peptide pull-down experiments (Figure 4A). Likewise, the C-terminal domain of UHRF1, but not the FYVE domain of Eea1, blocked TTD/H3K9me3 interaction in FP experiments (Figure 4B). Similar results were obtained for the TTD-PHD, TTD-PHD-SRA, and UBL-TTD-PHD-SRA recombinant fragments of UHRF1 (Figures S4C-S4E).

Titration of $\mathrm{H} 3 \mathrm{~K} 9 \mathrm{me} 3$, but not $\mathrm{H} 3 \mathrm{~K} 9 \mathrm{me} 0$ peptide, into an immunoprecipitation of the $\mathrm{C}$ terminus resulted in significantly reduced recovery of the TTD (Figure $4 \mathrm{C}$ ). These results were confirmed using only the PBR region of the C-terminal domain. In FP experiments, the PBR peptide blocked binding of the TTD to the H3K9me3 peptide (Figure 4D). Mutagenesis of the K644 and $\mathrm{K} 646$ residues that were not implicated in the binding of the PBR to the TTD peptide-binding groove had only a limited effect. While mutagenesis of the $\mathrm{K} 650$ residue somewhat reduced the blocking effect of the PBR peptide, changing the individual K648 or R649, or the combined R649, K650, and S651 sites, resulted in severe loss of blocking. Similar results were obtained for the TTD-PHD (Figure S4F).

Only the PBR, but not the much weaker binding linker peptide, blocked interaction of the TTD with H3K9me3 (Figure S4G). This effect was abrogated in the D142A mutant (Figure S4H). Moreover, the PBR peptide competed with the N-terminally labeled H3K9me3, but not the C-terminally labeled H3K9me3 or H3K9me0 peptides, for binding to the TTD-PHD (Figures S4I$\mathrm{S} 4 \mathrm{~K})$. The results supported the idea that binding of either the H3K9me3 or PBR peptides to the TTD are mutually exclusive and independent of linker function as well as PHD binding to the extreme $\mathrm{N}$ terminus of $\mathrm{H} 3$. Presence of an $\mathrm{H} 3 \mathrm{~K} 9$ me3 peptide compromised the interaction of the TTD (Figure 4E) or TTD-PHD (Figure S4L) with the PBR, while a H3K9me0 peptide had a much reduced effect.

Next, we designed multiple UHRF1 protein constructs with mutations in the PBR region. While mutagenesis of $\mathrm{K} 650$ had no effect, the R649A mutant showed increased binding to $\mathrm{H} 3 \mathrm{~K} 9 \mathrm{me} 3$ in FP experiments (Figure 4F). This effect was stronger when, in addition, the K650 and $\mathrm{S} 651$ or the K644, K646, K648, $\mathrm{K} 650$, and $\mathrm{S} 651$ residues were also mutated. The same mutations had only little effect on the interaction of UHRF1 with an H3K9me0 peptide (Figure S4M). Similarly, we found that the UHRF1 K644A, K646A, K648A, R649A, K650A,S651A mutant protein had increased binding to H3K9me3 in pull-down experiments (Figure 4G). While the results potentially implied that there are additional interfaces between the TTD and $C$ terminus, they supported a blocking effect of the PBR in the context of the fulllength UHRF1 protein.

\section{The PBR of UHRF1 Interacts with PI5P}

To isolate the small cellular cofactor of UHRF1, we set up a chromatographic purification scheme (Figures S5A and S5B). Dialyzed, recombinant UHRF1 and the associated factors from the HeLa NE (all presumably $<3 \mathrm{kDa}$ ) were separated by reversed-phase chromatography. Individual fractions were lyophilized and then tested for their ability to induce H3K9me3-specific binding of full-length recombinant UHRF1. The activating factor(s) could be separated by step-wise elution at 50\% acetonitrile from a C8 column (Figure S5C). While it was possible to further refine the purification scheme and to combine different chromatography methods, several attempts to identify the activating factors by mass spectrometry failed.

In order to gain insights into the nature of the compounds from another direction, we defined the region of UHRF1 binding the NE factor(s). To this end, different domains of UHRF1 were subjected to dialysis against HeLa NE. Material eluting at $50 \%$ acetonitrile in our purification scheme was analyzed in histone tail pull-down experiments with recombinant UHRF1. The fragments of UHRF1 containing the C-terminal domain (WT, UBL-TTD SRA-RING, C terminus), but not those without the C terminus (UBL-TTD SRA, UBL-TTD-PHD SRA), were able to recruit factor(s) that induced $\mathrm{H} 3 \mathrm{~K} 9 \mathrm{me} 3$-specific binding (Figure $5 \mathrm{~A}$ ). Indeed, dialysis of the $\mathrm{C}$ terminus against HeLa NE relieved its inhibition of the interaction between the TTD and H3K9me3 (Figure 5B), while dialysis of the TTD itself had no effect (Figure 5C).

Upon a closer look at the PBR sequence within the $C$ terminus, we realized that it contains a motif $\left(K / R-(X)_{n=3-7}-K-X-K / R-K / R\right)$ that had been implied in binding PIPs (Lewis et al., 2011). Indeed, treatment with phospholipase A2 (PLA2) that hydrolyzes PIPs abolished the capability of HeLa NE to activate H3K9me3-specific binding of recombinant UHRF1 (Figure 5D). We then tested recombinant UHRF1 for interaction with different PIPs, its precursors, and related compounds in standard lipid dot blot assays (Figures S5D and S5E). Similar to the PHD domain of ING1, UHRF1 bound PI5P in these assays (Bua and Binda, 2009). 


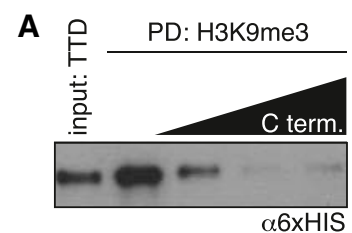

C

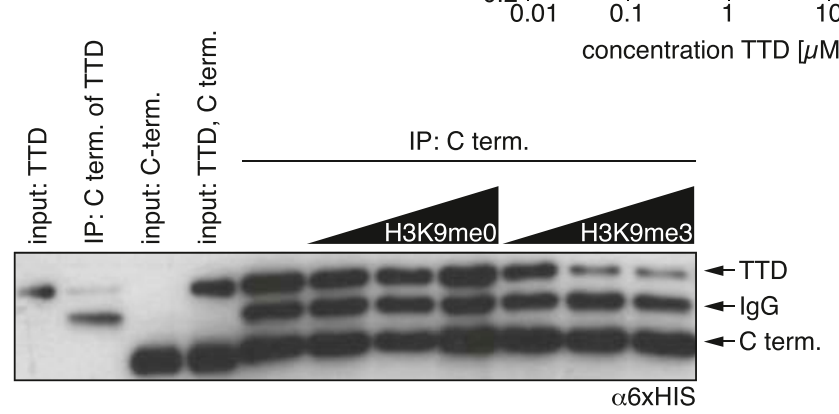

D

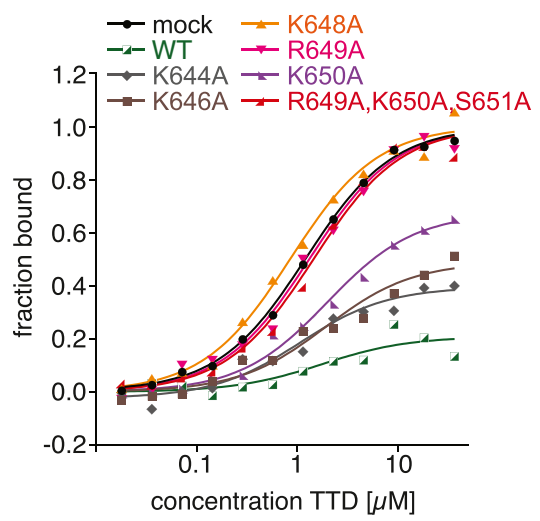

$\mathbf{F}$
B

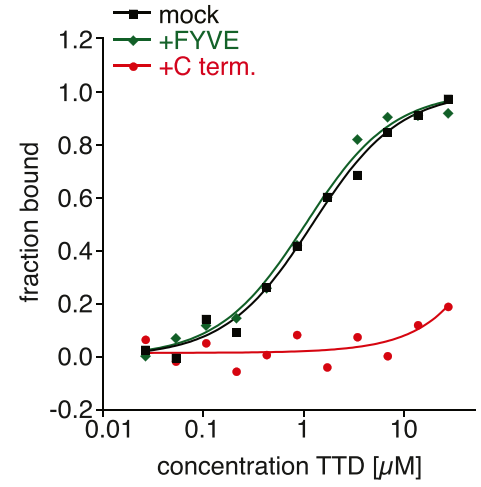

G

Figure 4. The PBR Blocks H3K9me3 Binding of the TTD

(A) TTD was incubated with increasing concentrations of the $\mathrm{C}$ terminus (molar ratio TTD:C term. $=2: 1,1: 1,1: 1.5$ ) and analyzed in pull-down (PD) experiments using an immobilized $\mathrm{H} 3 \mathrm{~K} 9 \mathrm{me} 3$ peptide. Recovered material was analyzed by western blot. Input, $2 \%$.

(B) TTD alone (mock) or in the presence of the $C$ terminus of UHRF1 or the FYVE domain of Eea1 (1:2 molar ratio) was analyzed in fluorescence polarization binding experiments with FAM$\mathrm{H} 3(1-15) K 9$ me3 peptide.

(C) C terminus was incubated with the TTD in the presence of increasing concentrations of the indicated $\mathrm{H} 3(1-20)$ peptides (molar ratio TTD:C term.:peptide $=1: 1: 1, \quad 1: 1: 2.5,1: 1: 5$, $1: 1: 7.5)$ and immunoprecipitated using antibodies against UHRF1. Recovered material was analyzed by western blot. Running positions of proteins recognized by the primary and/or secondary antibodies are indicated. Input, $5 \%$.

(D) TTD alone (mock) or in the presence of the indicated PBR wild-type (WT) or mutant peptides (1:5 molar ratio) was analyzed in fluorescence polarization binding experiments with FAM- $\mathrm{H} 3(1-$ 15)K9me3 peptide.

(E) TTD alone (mock) or in the presence of the indicated $\mathrm{H} 3(1-20)$ peptides (1:5 molar ratio) was analyzed in fluorescence polarization binding experiments with $\mathrm{FAM}-\mathrm{H} 3(1-15) \mathrm{K} 9 \mathrm{me} 3$ peptide.

(F) Wild-type (WT) UHRF1 or UHRF1 carrying the indicated mutations was analyzed in fluorescence polarization binding experiments with FAM- $\mathrm{H} 3(1-$ 15)K9me3 peptide.

(G) Mutant UHRF1 K644A,K646A,K648A,R649A, $\mathrm{K} 650 \mathrm{~A}, \mathrm{~S} 651 \mathrm{~A}$ was analyzed in a pull-down experiment using the specified biotinylated $\mathrm{H} 3(1-$ 20) peptides immobilized on streptavidin magnetic beads or beads alone (mock). Recovered material was analyzed by western blot. Input, $2 \%$. See also Figure S4 and Tables S1 and S2.
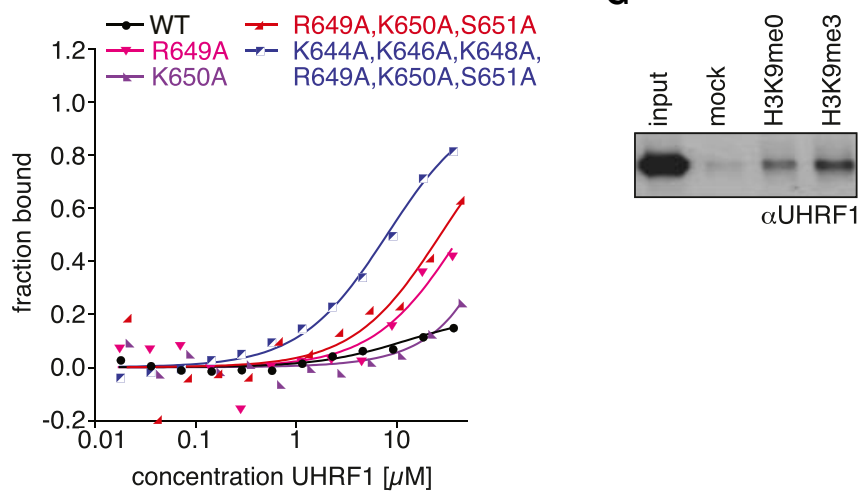

We then used a liposome flotation assay to further define the PIP binding properties of UHRF1 (Figure 5E) (Rusten and Stenmark, 2006). As expected, the PHD domain of ING1 was only retained by liposomes containing PI5P, but not any of the other PIPs, while the FYVE domain of Eea1 was only retained on liposomes containing PI3P (Figures 5F and S5F) (Simonsen et al., 1998). Some background binding of both factors to PIP-free liposomes was also seen. While UHRF1 showed a similar background with PIPfree liposomes, it was only specifically re-

As with ING1, additional interactions with other lipids (PI5P > $\mathrm{PI} 3 \mathrm{P}=\mathrm{PA}>\mathrm{PI}, 5 \mathrm{P}_{2}=\mathrm{PI} 4,5 \mathrm{P}_{2}>\mathrm{PS}$ ) were also observed. As anticipated, no interaction of the $\mathrm{H} 3 \mathrm{~K} 9$ me3-binding $\mathrm{HP} 1 \beta$ protein with any of the lipids was seen. tained on liposomes containing PI5P. No binding to liposomes containing PI3P, PI4P, PI3,5 $\mathrm{P}_{2}$, or $\mathrm{PI} 4,5 \mathrm{P}_{2}$ was observed.

Mapping of the interaction using lipid dot blots as well as liposome flotation assays defined the $\mathrm{C}$ terminus and, within that, 
the PBR-containing pre-RING region as the PI5P binding domain of UHRF1 (Figures 5G, S5E, and S5F). Mutagenesis of R649, $\mathrm{K} 650$, and $\mathrm{S} 651$ within the PBR was sufficient to severely reduce binding of full-length UHRF1 as well as the pre-RING region to PI5P. Lastly, we compared binding of fluorescently labeled wild-type and R649A,K650A,S651A mutant PBR peptides to biotinylated PIPs immobilized on streptavidin beads (Figures $5 \mathrm{H}$ and $\mathrm{S} 5 \mathrm{G})$. A clear preference of the wild-type PBR for PI5P over PI, PI3P, PI4P, PI3,5P 2 , and $\mathrm{PI} 4,5 \mathrm{P}_{2}$ was observed.

\section{PI5P Regulates UHRF1 Interaction with the Modified or Unmodified H3 Tail}

When titrated into immunoprecipitations of the C-terminal domain with the TTD, PI5P, but not PI4P, blocked the interaction (Figure 6A). Similarly, PI5P, but not PI4P, reduced the recovery of the TTD on the PBR peptide in pull-down experiments (Figure 6B).

We then asked whether $\mathrm{PI5P}$ could modulate $\mathrm{H} 3$ tail binding of UHRF1. In histone pull-down experiments, PI5P induced H3K9me3-specific binding of recombinant UHRF1 (Figure 6C). While PI3P also showed some activation, neither PA, PI, PI4P, $\mathrm{PI} 3,5 \mathrm{P}_{2}$, nor $\mathrm{PI}, 5 \mathrm{P}_{2}$ had an effect. All attempts with PI5P containing different acyl chain lengths to induce $\mathrm{H} 3 \mathrm{~K} 9$ me3-specific binding of UHRF1 in the FP-based experiments in solution failed. Also, the isolated inositolphosphate head groups of the PIPs had no effect (not shown).

To further investigate whether PI5P is indeed the cellular molecule regulating UHRF1, we made use of a specific kinase. PIP4K $\alpha$ transduces together with PIP4K $\beta$ to the cell nucleus and phosphorylates PI5P on the 4-position, thereby yielding $\mathrm{PI}_{4}, 5 \mathrm{P}_{2}$ (Bultsma et al., 2010). Addition of PIP4K $\alpha$ and ATP to an H3 tail pull-down reaction of recombinant UHRF1 abolished the activating function of PI5P (Figure S6). ATP alone had no effect, and the kinase did not alter the outcome with PI4P in this assay. We then incubated HeLa NE or a buffer control with PIP4K $\alpha$ and either ATP or the nonhydrolysable ATP $\gamma \mathrm{S}$. UHRF1 was dialyzed against the treated lysate or buffer and analyzed in $\mathrm{H} 3$ tail pulldown experiments. Pretreatment of the HeLa NE with ATP and PIP4K $\alpha$ abolished the activating effect of the lysate (Figure 6D). Incubation of the HeLa NE with ATP alone or PIP4K $\alpha$ and ATP $\gamma S$ also slightly induced some H3K9me3-specific binding of UHRF1. In contrast, pretreatment with ATP $\gamma S$ had no effect. We think ATP and PIP kinase(s) present in the NE might account for these observations. No effect was observed with the buffer control, and there was no differential binding of the recombinant UHRF1 to $\mathrm{H} 3 \mathrm{~K} 9 \mathrm{meO}$ and $\mathrm{H} 3 \mathrm{~K} 9 \mathrm{me} 3$. Altogether, the results indicated that $\mathrm{PI5P}$ is a cellular coactivator of UHRF1, allosterically regulating binding to different modification states of the $\mathrm{H} 3$ tail.

\section{PI5P Directs TTD-Dependent UHRF1 Localization to Heterochromatin}

Previous studies have found both strong and limited localization of UHRF1 to H3K9me3-enriched heterochromatin (Karagianni et al., 2008; Nady et al., 2011; Papait et al., 2007). We hypothesized that these discrepancies might be due to different cellular systems and conditions analyzed. We found that a large fraction (58\%) of NIH 3T3 cells transiently expressing
mCherry-UHRF1 showed colocalization of the fusion protein with DAPI-dense and H3K9me3-positive foci of pericentromeric heterochromatin. Lower enrichment at these regions (intermediate) was seen for $29 \%$ of cells, while $13 \%$ of cells displayed diffuse nuclear distribution (Figure S7A). Mutation of two residues in the aromatic cage implicated in methyl-lysine binding in the TTD/H3K9me3 complex resulted in significantly more cells (64\%) showing intermediate and diffuse phenotypes, while $36 \%$ of cells showed dotted UHRF1 Y188A,Y191A distribution (Figure S7B). The results suggested that other domains of the protein besides the TTD are also involved in heterochromatin localization. Indeed and in agreement with recent findings (Liu et al., 2013), after mutation of both the TTD and SRA domains, no heterochromatin localization of mCherry-UHRF1 was detected (Figure S7C). While the exact regulation of UHRF1 subnuclear and chromatin association is unclear, the protein might rely on different domains for target binding, and there could be a cell-cycle dependency (Papait et al., 2007).

To determine the regulatory role of the PBR, we coexpressed GFP-tagged wild-type or mutant (K644A, K646A, K648A, R649A, K650A,S651A) pre-RING domain together with mCherryUHRF1 in NIH 3T3 cells (Figures 7A and S7D). We thought this region might exert dual effects by simultaneously interfering with TTD/H3K9me3 binding as well as by titrating endogenous PI5P. Quantification of the different phenotypes indicated that an average of $12 \%$ of cells exhibited a shift in their mCherryUHRF1 distribution from dotted to intermediate or diffuse appearance upon expression of wild-type GFP-pre-RING. This effect was not seen for coexpression of mCherry-UHRF1 with the mutant GFP-pre-RING (Figures 7B and S7E).

To further establish a regulatory role of PI5P for UHRF1, we made use of the PIP4K kinases to manipulate nuclear levels of the cofactor. Consistent with the phenotypes of pre-RING overexpression, coexpression with MYC-PIP4K $\alpha$ and PIP4K $\beta$ (PIP4K WT) relative to coexpression of empty vector resulted in an average of $9 \%$ fewer cells displaying the nuclear dotted pattern of mCherry-UHRF1. In contrast, kinase mutant PIP4Ks (PIP4K $\alpha$ G131L,Y138F and PIP4K $\beta$ D278A) had no such effect (Figures $7 \mathrm{C}$ and S7F-S7H). Given that only a fraction of cells in the population (on average $22 \%$ ) relied on the TTD for localization to pericentric heterochromatin, we deduced from these experiments that PI5P binding to the PBR has a marked impact ( $40 \%$ ) on heterochromatin association of this UHRF1 subpopulation.

\section{DISCUSSION}

Regulation of UHRF1 H3 Tail Binding by Conformational Rearrangements Allosterically Induced by PI5P

By identifying a mode of regulation of UHRF1 by a cellular cofactor, our findings clarify discrepancies in the literature regarding the binding activities and domain usage of this important epigenetic regulator. Various reports used different experimental systems (i.e., protein expression in bacteria, which do not have PIPs, versus expression in insect cells, reticulocyte lysate, or mammalian cells, which all contain PIPs) and investigated full-length, deletion mutants, or point mutants of UHRF1 proteins. Distinct domains were implicated in binding to 
A

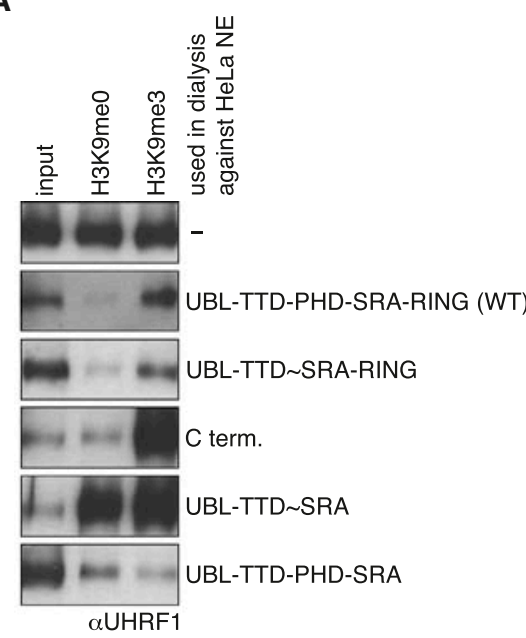

D

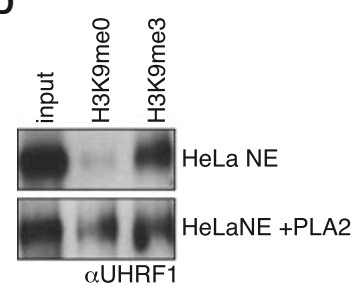

E

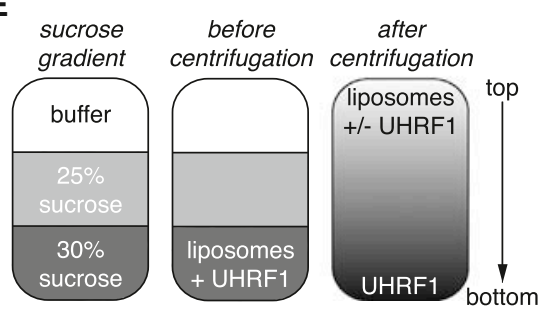

B

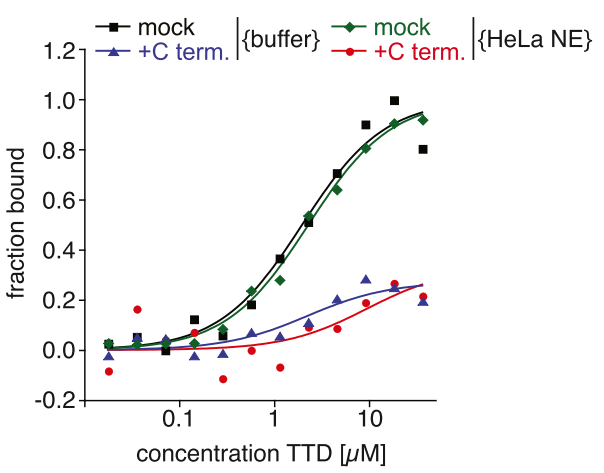

C

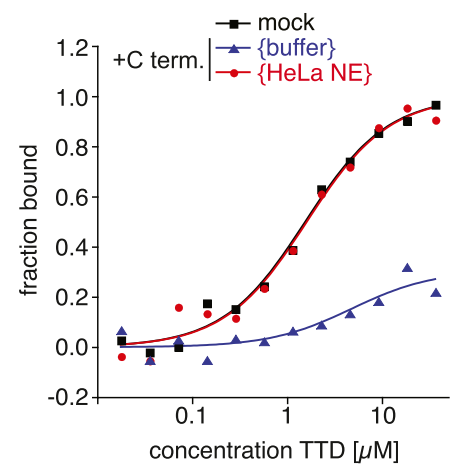

\section{$\mathbf{F}$}

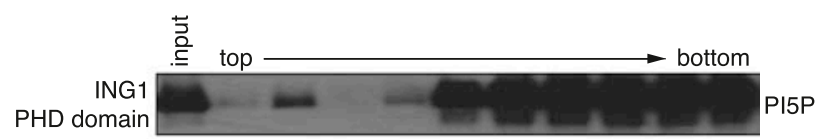

aGST

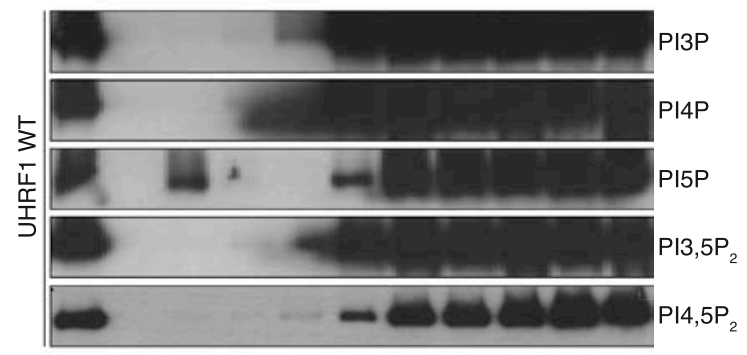

UHRF1

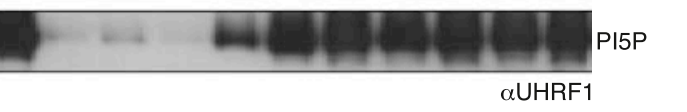

H

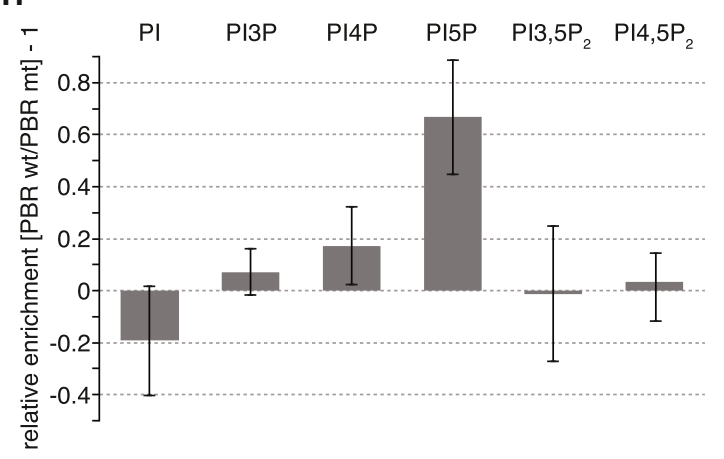

G

\begin{tabular}{|r|c|c|c|c|c|c|c|}
\hline liposomes & - & + & + & + & + & + & + \\
PIP & - & - & $\mathrm{PI3P}$ & $\mathrm{PI} 4 \mathrm{P}$ & $\mathrm{PI} 5 \mathrm{P}$ & $\mathrm{PI}_{3,5 \mathrm{P}_{2}}$ & $\mathrm{PI}_{4} 5 \mathrm{P}_{2}$ \\
\hline UHRF1 & - & $-/+$ & - & - & + & - & - \\
C term. & - & - & $-/+$ & - & + & - & nd \\
pre-RING & nd & $-/+$ & $-/+$ & - & + & nd & nd \\
$\Delta$ C term. & nd & - & - & - & - & - & nd \\
PHD & nd & - & - & - & - & - & nd \\
UHRF1 MT & - & $-l+$ & nd & nd & - & nd & nd \\
Pre-RING MT & - & $-/+$ & nd & nd & - & nd & nd \\
\hline Eea1 FYVE domain & - & - & + & - & - & nd & nd \\
ING1 PHD domain & nd & - & $-/+$ & - & + & - & nd \\
\hline
\end{tabular}

Figure 5. The PBR of UHRF1 Interacts with PI5P

(A) The indicated fragments of UHRF1 were dialyzed against HeLa NE. Proteins and cellular factors inside the dialysis membrane were separated on a C8 reversed-phase column. Recombinant $6 \times$ HIS-UHRF1 was incubated with the material eluting at $50 \%$ acetonitrile and probed in a histone peptide pull-down experiment. Recovered material was analyzed by western blot. Input, $2 \%$.

(B) TTD alone (mock) or incubated with the $C$ terminus dialyzed against HeLa NE or buffer control (molar ratio TTD:C term. $=1: 2$ ) was analyzed in fluorescence polarization binding experiments using FAM-H3(1-15)K9me3 peptide.

(C) TTD was dialyzed against buffer or HeLa NE. Protein alone (mock) or in the presence of the $\mathrm{C}$ terminus was analyzed in fluorescence polarization binding experiments using FAM-H3(1-15)K9me3 peptide. 
A

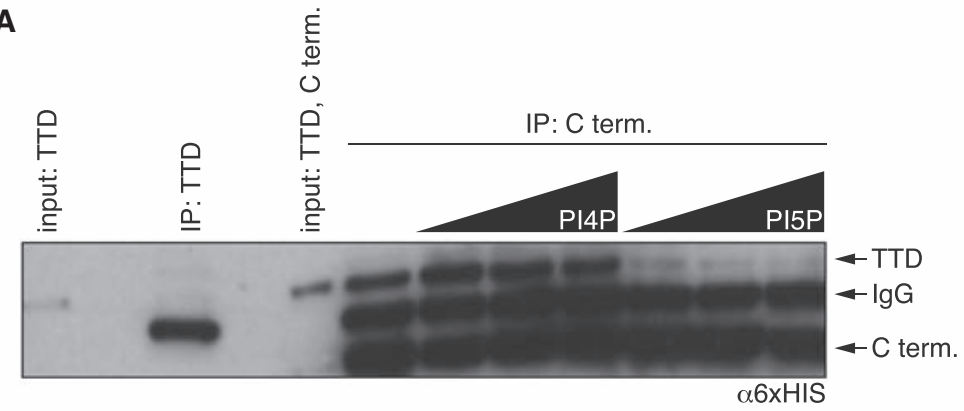

B

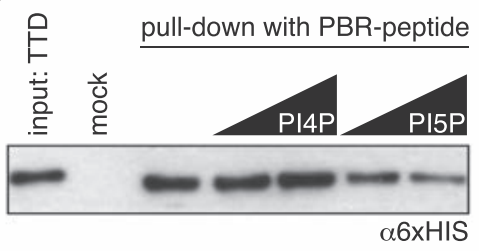

C

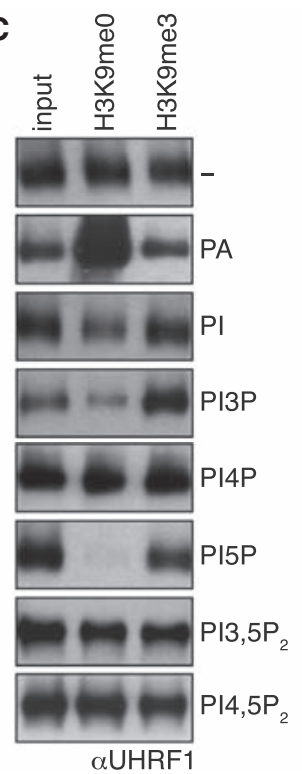

D
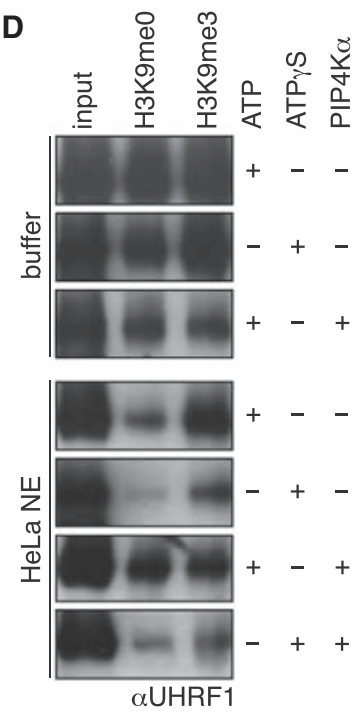

Figure 6. PI5P Releases Blocking of the TTD by the C Terminus

(A) C terminus was incubated with TTD in the presence of increasing concentrations of the indicated PIPs (molar ratio TTD:C term.:PIP = 1:1:5, 1:1:10, 1:1:20) and immunoprecipitated using antibodies against UHRF1. Recovered material was analyzed by western blot. Running positions of proteins recognized by the primary and/or secondary antibodies are indicated. Input, $5 \%$.

(B) TTD was incubated with increasing concentrations of the indicated PIPs (molar ratio TTD:PIP = 1:5, 1:10) and analyzed in pull-down experiments using an immobilized PBR peptide. Recovered material was analyzed by western blot. Mock, pull-down with beads only; input, $2 \%$.

(C) UHRF1 in the presence of the indicated lipids (2-fold molar excess) was incubated with the specified biotinylated H3(1-20) peptides immobilized on streptavidin magnetic beads. Recovered material was analyzed by western blot. Input, $2 \%$.

(D) Recombinant UHRF1 was dialyzed against buffer or HeLa NE that was incubated with the indicated combinations of PIP4K $\alpha$, ATP, or ATP $\gamma$ S. After dialysis, the protein was probed in histone peptide pull-down reactions. Recovered material was analyzed by western blot. Input, $2 \%$. See also Figure S6.

unmodified and $\mathrm{K} 9 \mathrm{me} 3 \mathrm{H} 3$, and different interaction specificities were observed (Hu et al., 2011; Karagianni et al., 2008; Liu et al., 2013; Rajakumara et al., 2011; Rottach et al., 2010; Wang et al., 2011). We propose that most of the differences can be explained on the basis of the following regulatory mechanism. In the ground state of the recombinant protein or highly purified cellular protein, the C-terminal region of UHRF1 is folded back onto the middle region with the PBR sequence bound to the peptidebinding groove of the TTD (Figure 7D, top). This prevents the interaction of the TTD-PHD linker and also the H3 N-terminal tail with the TTD (Figure 4) (Nady et al., 2011). In this state, the PHD domain is able to bind to the extreme unmodified $\mathrm{N}$ terminus of H3. In an intermediate state that is artificially stabilized by mutagenesis of the PBR, the blockage of the TTD is released (Figure 7D, middle). We think this conformation of UHRF1 reflects the cooperative mode that we and others have observed for the isolated TTD-PHD fragment (Arita et al., 2012; Cheng et al., 2013; Rothbart et al., 2013; Xie et al., 2012). It is characterized by a slight preference for $\mathrm{H} 3 \mathrm{~K} 9 \mathrm{me} 3$ over the unmodified state, as seen for C-terminally labeled peptides $(\mathrm{H} 3[1-15]-\mathrm{K}$ [FAM]). Here, the linker sequence occupies the peptide-binding groove of the TTD. The $\mathrm{N}$ terminus of the $\mathrm{H} 3$ tail can bind to the $\mathrm{PHD}$, while the aromatic cage of the TTD can recognize K9me3. In an activated state, the PBR is bound by PI5P, further stabilizing the domain orientation, giving TTD access to H3K9me3. Large conformational rearrangements not only free the TTD for H3K9me3 binding, but also block the PHD from binding the extreme unmodified $\mathrm{N}$ terminus of H3 (Figure 7D, bottom).

Further biophysical and structural studies will be needed to clarify the exact nature of the overall conformational rearrangements of the protein in the different states as well as the exact binding modes of the $\mathrm{H} 3$ tail and PI5P to UHRF1. The

(D) Recombinant UHRF1 was dialyzed against untreated HeLa NE or HeLa NE that was incubated with phospholipase A2. After dialysis, the protein was probed in a histone peptide pull-down experiment. Recovered material was analyzed by western blot. Input, $2 \%$.

(E) Scheme of liposome flotation assay. The sucrose gradient was loaded with liposomes and UHRF1. After centrifugation, the gradient was fractionated and analyzed for localization of UHRF1.

(F) The indicated proteins were incubated with liposomes containing the indicated PIPs. Fractions of the liposome flotation assay after centrifugation were analyzed by western blot. WT, wild-type; input, $5 \%$.

(G) Summary of multiple liposome flotation assays using different proteins. MT, mutation R649A,K650A,S651A. +, major protein signal at the top of the gradient; -/+, minor protein signal at the top of the gradient; nd, not determined.

(H) Fluorescein-labeled wild-type (WT) or R649A,K650A,S651A mutant (MT) PBR peptides were incubated with the indicated biotinylated PIPs immobilized in 96well plates. Fluorescence signals after washing were recorded. Averaged ratio of enrichment of WT versus MT of four independent experiments is plotted. Error bars correspond to error propagation of SEM reflected in five independent experiments. See also Figure S5 and Tables S1 and S2. 
A

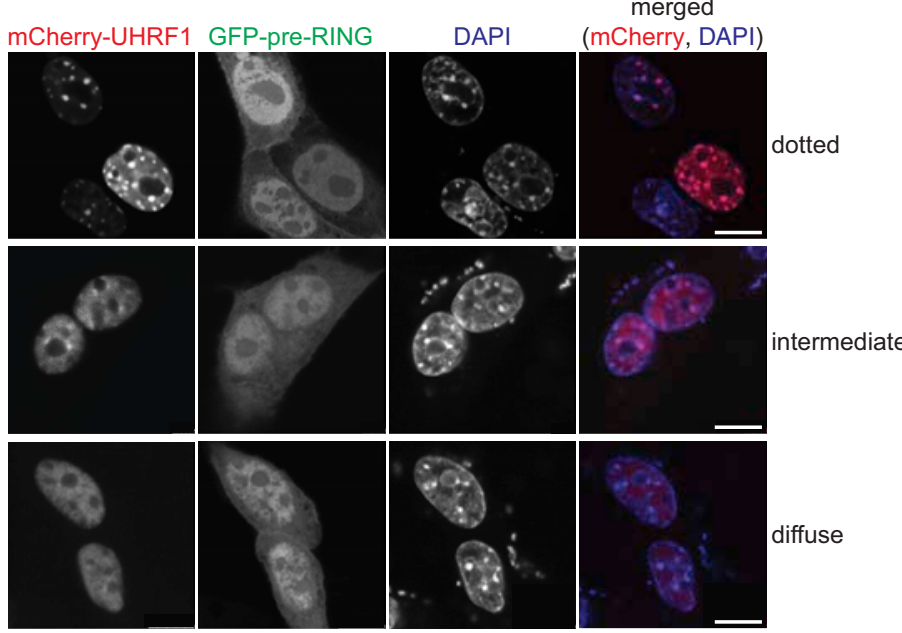

C

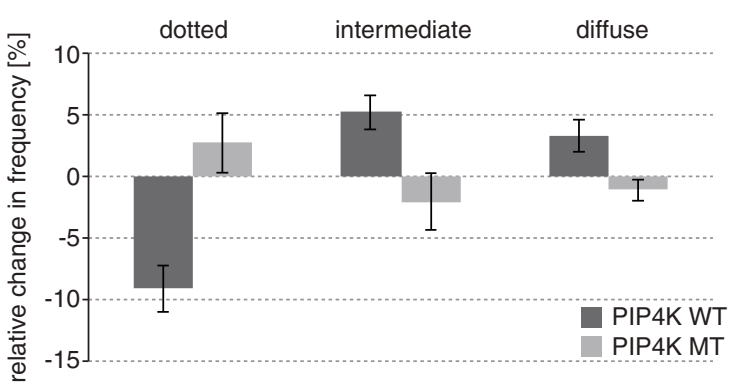

B

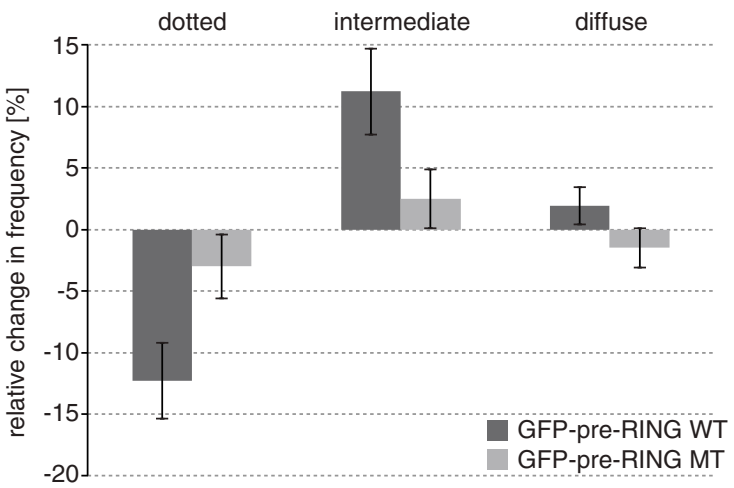

D
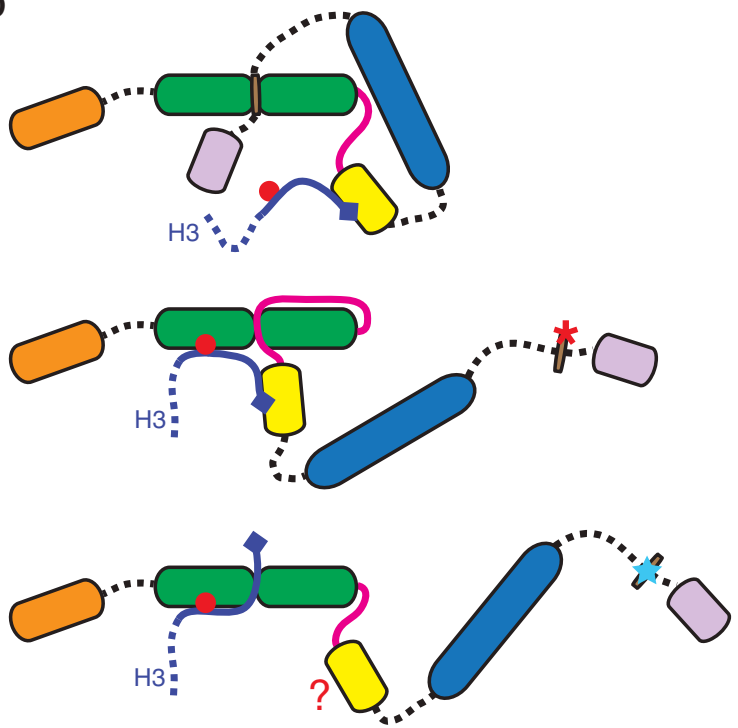

Figure 7. Nuclear PI5P Regulates Heterochromatin Association of UHRF1

(A) NIH 3T3 cells were transfected with mCherry-UHRF1 together with GFP-pre-RING. Fluorescence signals were analyzed by confocal microscopy. Images of representative cells of different phenotypes observed at the indicated frequencies $(n>500)$ are shown. DAPI was used to stain DNA. Scale bar, $10 \mu \mathrm{m}$.

(B) Histogram showing changes in frequencies of observed nuclear distribution of mCherry-UHRF1 when coexpressing GFP-pre-RING wild-type (WT) or GFPpre-RING K644A,K646A,K648A,R649A,K650A,S651A (MT) relative to coexpression of a GFP control in NIH 3T3 cells as classified in (A) ( $n>500)$. Error bars represent error propagation of SEM reflected in four independent experiments.

(C) Histograms showing changes in frequencies of observed nuclear distribution of mCherry-UHRF1 coexpressing MYC-PIP4K $\alpha$ and untagged PIP4K $\beta$ (PIP4K WT) or MYC-PIP4K $\alpha$ G131L,Y138F and untagged PIP4K $\beta$ D278A (PIP4K MT) relative to coexpression of empty vector in NIH 3 T3 cells as classified in (A) ( $\mathrm{n}>500$ ). Error bars represent error propagation of SEM reflected in four independent experiments.

(D) Different conformational states of UHRF1. Top: the PHD mediates binding to unmodified extreme N terminus of $\mathrm{H} 3$ in the absence of PI5P, and the TTD is blocked from binding H3K9me3 by the PBR. Middle: when the PBR is mutated (red asterisk), it is dissociated from the TTD. The linker can mediate cooperative binding of the $\mathrm{H} 3$ tail by the PHD and TTD domains. Bottom: PI5P (blue star) binding by the PBR results in conformational rearrangement. The TTD is free to bind the H3K9me3 tail. Unknown mechanisms (indicated by the question mark) block the PHD from binding the unmodified extreme $\mathrm{N}$ terminus of $\mathrm{H} 3$. See also Figure $\mathrm{S} 7$.

mechanism of PHD blocking in the activated state is particularly unclear at this point. Our data question whether the cooperative state seen for the TTD-PHD exists in the context of the fulllength protein. It might be an artificial state of an isolated fragment. The increase seen in H3K9me3 binding of TTD-PHD over TTD varies from 2-fold (this study; Arita et al., 2012) to 5-fold (Cheng et al., 2013; Rothbart et al., 2013), which might be due to different experimental conditions. In all cases and likely due to the flexible linkage of the TTD and PHD, it is not sufficient to account for the large binding differences for the unmodified and K9me3 H3 tail observed for cellular UHRF1.
Whether the slight preference for the K9me3 over the unmodified $\mathrm{H} 3$ peptide with $\mathrm{C}$-terminal fluorescein $(\mathrm{H} 3[1-15]-K[F A M]$; Figure 1 and Table S1) reflects cooperativity of TTD and PHD in the ground state remains to be seen.

How are the hydrophobic acyl chains of PI5P bound to UHRF1 outside of a lipid membrane or micellar structure? While mutagenesis of the PBR is sufficient to abolish interaction in the lipid flotation assay (Figure 5F), we think that other regions of the $C$ terminus and/or additional unknown components are involved in PI5P binding. First, in the liposome flotation assay, only the polar head groups of the PIPs are exposed; the hydrophobic 
acyl chains are embedded in the lipid layer. Nevertheless, inositol 1,5-bisphosphate is not sufficient to induce allosteric activation of UHRF1. Second, while addition of PI5P to UHRF1 activates the protein in pull-down assays, it does not have this effect in solution FP assays (data not shown). We speculate that the phospholipid might get artificially enriched on the surface of the beads used in the pull-down assays. In the nuclear extract, PI5P is bound by UHRF1 and likely other factors that might work as "sinks" for the PIP. For sufficient transfer across the dialysis membrane, PI5P might need other small molecules (e.g., ions, peptides, RNA, etc.) that stabilize it in solution.

\section{Cellular Regulation of UHRF1}

While our findings identify a role for PI5P in modulating UHRF1 function, regulation of the protein in the cellular context is likely more complicated. Recent work had reported partially overlapping and redundant roles of the TTD and SRA domains in UHRF localization, where the two regions appear to mediate crosstalk between $\mathrm{H} 3 \mathrm{~K} 9$ methylation and DNA methylation at the level of DNA methylation maintenance (Liu et al., 2013). In contrast, another report suggested that cooperative interplay of the TTD and PHD is required for this function (Rothbart et al., 2013). The fact that the concentration of PI5P in the nuclei of murine erythroleukemia cells increases 20-fold during G1 phase but drops after S phase (Clarke et al., 2001) could indicate that switching between the unmodified $\mathrm{H} 3$-binding ground state and the activated $\mathrm{H} 3 \mathrm{~K} 9$ me3-binding state of UHRF1 is required for differential localization of the protein during the cell cycle (Papait et al., 2007). Further work will need to clarify the precise crosstalk of the chromatin modification binding TTD, PHD, and SRA domains of UHRF1 under defined cellular conditions. Regulation of $\mathrm{H} 3 \mathrm{~K} 9 \mathrm{me} 3$ and unmodified $\mathrm{H} 3$ tail interaction by PI5P also likely has an impact on UHRF1 binding to and targeting of its enzymatic partners such as G9a, DNMT1, and HDAC1.

Another level of regulation of UHRF1 is provided by posttranslational modifications. Several proteomics studies have defined serine phosphorylation events within the linker and C-terminal region. Also, acetylation of two lysine residues within the SRA was found (Choudhary et al., 2009; Dephoure et al., 2008; Olsen et al., 2010; Rigbolt et al., 2011). While the functions of most modifications are unknown, phosphorylation by CDK1 at $\mathrm{S} 639$ is involved in degradation of the protein (Ma et al., 2012). Phosphorylation of S298 within the linker by PKA interferes with TTD-PHD cooperativity in vitro (Arita et al., 2012). Based on our mutagenesis studies of the PBR, we speculate that phosphorylation of $\mathrm{S} 651$ (S651 ph) might have a regulatory role in PI5P binding and in modulating $\mathrm{H} 3$ interaction of UHRF1 (Rigbolt et al., 2011).

We predict that PI5P, as well as other nuclear PIPs, might have more general, so far unrecognized roles in directly regulating the chromatin binding activity of different proteins. Consistent with a role in signaling, the amounts of certain nuclear PIPs are increased in mammalian cells by physiological ligands or processes as well as by cellular stresses (Shah et al., 2013). This might directly impact gene regulation. In general, nuclear phospholipids undergo changes in abundance that match the transcriptional activity during the cell cycle (Fraschini et al., 1999). Defining further chromatin proteins that are directly regulated by PIPs or other phospholipids might establish new paradigms of signal transduction in the cell nucleus.

\section{EXPERIMENTAL PROCEDURES}

\section{Reagents}

Detailed listing of plasmids, peptides, antibodies, and proteins can be found in the Supplemental Information.

\section{Dialysis of UHRF1}

Nuclear extracts were prepared as described (Dignam et al., 1983). Protein concentration ranged from $10-15 \mathrm{mg} / \mathrm{ml}$. Proteins $(5 \mathrm{mg}$ in $400 \mu \mathrm{l})$ were dialyzed against $10 \mathrm{ml} \mathrm{HeLa} \mathrm{NE}$ or comparable buffer (in $50 \mathrm{ml}$ tubes) overnight in dialysis cups (Pierce) with a 3,500 Da molecular weight cutoff at $4^{\circ} \mathrm{C}$.

Pull-Downs, Immunofluorescence and Fluorescence Polarization, and Liposome Flotation Assay

Experiments were performed as previously described (Fischle et al., 2008; Rusten and Stenmark, 2006), with minor modifications as can be found in the Supplemental Experimental Procedures.

Binding of PBR Peptides to PIP Beads

A $10 \mu \mathrm{l}$ slurry of PIP beads (Echelon Biosciences) was incubated with $20 \mathrm{ng}$ of fluorescein-labeled PBR peptide. After washing, samples were transferred to black 96-well plates (Corning), and fluorescence intensity (excitation at $485 \mathrm{~nm}$, emission at $535 \mathrm{~nm}$ ) was measured in a HIDEX Chameleon II plate reader. Results from three successive reads were averaged, and recovery of the peptides was normalized relative to the input.

\section{NMR Spectroscopy}

Chemical shift changes of the residues of the TTD upon titration of unlabeled linker peptide were mapped using prior available chemical shift assignments deposited in the Biological Magnetic Resonance Bank (BMRB) (Nady et al., 2011).

Molecular Docking

Docking of the PBR peptide to the TDD was performed using HADDOCK v2.1 software (de Vries et al., 2007; Dominguez et al., 2003).

\section{SUPPLEMENTAL INFORMATION}

Supplemental Information includes Supplemental Experimental Procedures, seven figures, and two tables and can be found with this article online at http://dx.doi.org/10.1016/j.molcel.2014.04.004.

\section{ACKNOWLEDGMENTS}

We thank members of the Fischle lab for invaluable comments and discussions. Katharina Rehn helped with peptide synthesis, Nadin Zimmermann with MS analysis of peptides, and Sven Johannsson with quantitative analysis of cellular phenotypes. We are thankful to Thomas Conrad for fermenter cell culture and Christer Ejsing (University of Southern Denmark) for PIP MS work. K.A.G. was supported by an EMBO long-term fellowship; K.H.-H. is supported by a Marie Curie Fellowship (IEF, FP7). This work was funded by the Max Planck Society (W.F.), the German Research Foundation (DFG grants FI 1513/2-1 to W.F. and SCHW 1163/3-1 to D.S.), Cancer Research UK (N.D.), and the Natural Sciences and Engineering Research Council of Canada (grant 372475-10 to C.H.A.). C.H.A. holds a Canada Research Chair in Structural Genomics. The SGC is a registered charity (number 1097737) that receives funds from AbbVie, Boehringer Ingelheim, the Canada Foundation for Innovation, the Canadian Institutes for Health Research, Genome Canada through the Ontario Genomics Institute [OGI-055], GlaxoSmithKline, Janssen, Lilly Canada, the Novartis Research Foundation, the Ontario Ministry Research and Innovation, Pfizer, Takeda, and the Wellcome Trust (092809/Z/10/Z). 
Received: February 5, 2014

Revised: March 11, 2014

Accepted: April 2, 2014

Published: May 8, 2014

\section{REFERENCES}

Arita, K., Isogai, S., Oda, T., Unoki, M., Sugita, K., Sekiyama, N., Kuwata, K., Hamamoto, R., Tochio, H., Sato, M., et al. (2012). Recognition of modification status on a histone $\mathrm{H} 3$ tail by linked histone reader modules of the epigenetic regulator UHRF1. Proc. Natl. Acad. Sci. USA 109, 12950-12955.

Barlow, C.A., Laishram, R.S., and Anderson, R.A. (2010). Nuclear phosphoinositides: a signaling enigma wrapped in a compartmental conundrum. Trends Cell Biol. 20, 25-35.

Berkyurek, A.C., Suetake, I., Arita, K., Takeshita, K., Nakagawa, A., Shirakawa, M., and Tajima, S. (2014). The DNA methyltransferase Dnmt1 directly interacts with the SET and RING finger-associated (SRA) domain of the multifunctional protein Uhrf1 to facilitate accession of the catalytic center to hemi-methylated DNA. J. Biol. Chem. 289, 379-386.

Bostick, M., Kim, J.K., Estève, P.O., Clark, A., Pradhan, S., and Jacobsen, S.E. (2007). UHRF1 plays a role in maintaining DNA methylation in mammalian cells. Science 317, 1760-1764.

Bronner, C., Fuhrmann, G., Chédin, F.L., Macaluso, M., and Dhe-Paganon, S. (2010). UHRF1 Links the Histone code and DNA Methylation to ensure Faithful Epigenetic Memory Inheritance. Genet Epigenet 2009, 29-36.

Bua, D.J., and Binda, O. (2009). The return of the INGs, histone mark sensors and phospholipid signaling effectors. Curr. Drug Targets 10, 418-431.

Bua, D.J., Martin, G.M., Binda, O., and Gozani, O. (2013). Nuclear phosphatidylinositol-5-phosphate regulates ING2 stability at discrete chromatin targets in response to DNA damage. Sci Rep 3, 2137.

Bultsma, Y., Keune, W.J., and Divecha, N. (2010). PIP4Kbeta interacts with and modulates nuclear localization of the high-activity Ptdlns5P-4-kinase isoform PIP4Kalpha. Biochem. J. 430, 223-235.

Burgio, G., Onorati, M.C., and Corona, D.F. (2010). Chromatin remodeling regulation by small molecules and metabolites. Biochim. Biophys. Acta 1799, 671-680.

Catimel, B., Yin, M.X., Schieber, C., Condron, M., Patsiouras, H., Catimel, J., Robinson, D.E., Wong, L.S., Nice, E.C., Holmes, A.B., and Burgess, A.W. (2009). PI(3,4,5)P3 Interactome. J. Proteome Res. 8, 3712-3726.

Cheng, J., Yang, Y., Fang, J., Xiao, J., Zhu, T., Chen, F., Wang, P., Li, Z., Yang, H., and Xu, Y. (2013). Structural insight into coordinated recognition of trimethylated histone $\mathrm{H} 3$ lysine 9 (H3K9me3) by the plant homeodomain (PHD) and tandem tudor domain (TTD) of UHRF1 (ubiquitin-like, containing PHD and RING finger domains, 1) protein. J. Biol. Chem. 288, 1329-1339.

Choudhary, C., Kumar, C., Gnad, F., Nielsen, M.L., Rehman, M., Walther, T.C., Olsen, J.V., and Mann, M. (2009). Lysine acetylation targets protein complexes and co-regulates major cellular functions. Science 325, 834-840.

Clarke, J.H., Letcher, A.J., D'santos, C.S., Halstead, J.R., Jr., Irvine, R.F., and Divecha, N. (2001). Inositol lipids are regulated during cell cycle progression in the nuclei of murine erythroleukaemia cells. Biochem. J. 357, 905-910.

de Vries, S.J., van Dijk, A.D., Krzeminski, M., van Dijk, M., Thureau, A., Hsu, V., Wassenaar, T., and Bonvin, A.M. (2007). HADDOCK versus HADDOCK: new features and performance of HADDOCK2.0 on the CAPRI targets. Proteins $69,726-733$

Dephoure, N., Zhou, C., Villén, J., Beausoleil, S.A., Bakalarski, C.E., Elledge, S.J., and Gygi, S.P. (2008). A quantitative atlas of mitotic phosphorylation. Proc. Natl. Acad. Sci. USA 105, 10762-10767.

Dignam, J.D., Lebovitz, R.M., and Roeder, R.G. (1983). Accurate transcription initiation by RNA polymerase II in a soluble extract from isolated mammalian nuclei. Nucleic Acids Res. 11, 1475-1489.
Dominguez, C., Boelens, R., and Bonvin, A.M. (2003). HADDOCK: a proteinprotein docking approach based on biochemical or biophysical information. J. Am. Chem. Soc. 125, 1731-1737.

Fischle, W. (2012). One, two, three: how histone methylation is read. Epigenomics 4, 641-653.

Fischle, W., Franz, H., Jacobs, S.A., Allis, C.D., and Khorasanizadeh, S. (2008). Specificity of the chromodomain $Y$ chromosome family of chromodomains for lysine-methylated ARK(S/T) motifs. J. Biol. Chem. 283, 19626-19635.

Fiume, R., Keune, W.J., Faenza, I., Bultsma, Y., Ramazzotti, G., Jones, D.R., Martelli, A.M., Somner, L., Follo, M.Y., Divecha, N., and Cocco, L. (2012). Nuclear phosphoinositides: location, regulation and function. Subcell. Biochem. 59, 335-361.

Fraschini, A., Biggiogera, M., Bottone, M.G., and Martin, T.E. (1999). Nuclear phospholipids in human lymphocytes activated by phytohemagglutinin. Eur. J. Cell Biol. 78, 416-423.

Gozani, O., Karuman, P., Jones, D.R., Ivanov, D., Cha, J., Lugovskoy, A.A., Baird, C.L., Zhu, H., Field, S.J., Lessnick, S.L., et al. (2003). The PHD finger of the chromatin-associated protein ING2 functions as a nuclear phosphoinositide receptor. Cell 114, 99-111.

$\mathrm{Hu}$, L., Li, Z., Wang, P., Lin, Y., and Xu, Y. (2011). Crystal structure of PHD domain of UHRF1 and insights into recognition of unmodified histone $\mathrm{H} 3$ arginine residue 2. Cell Res. 21, 1374-1378.

Jones, D.R., Bultsma, Y., Keune, W.J., Halstead, J.R., Elouarrat, D., Mohammed, S., Heck, A.J., D'Santos, C.S., and Divecha, N. (2006). Nuclear Ptdlns5P as a transducer of stress signaling: an in vivo role for PIP4Kbeta. Mol. Cell 23, 685-695.

Karagianni, P., Amazit, L., Qin, J., and Wong, J. (2008). ICBP90, a novel methyl $\mathrm{K} 9 \mathrm{H} 3$ binding protein linking protein ubiquitination with heterochromatin formation. Mol. Cell. Biol. 28, 705-717.

Lallous, N., Legrand, P., McEwen, A.G., Ramón-Maiques, S., Samama, J.P., and Birck, C. (2011). The PHD finger of human UHRF1 reveals a new subgroup of unmethylated histone $\mathrm{H} 3$ tail readers. PLoS ONE 6, e27599.

Lewis, A.E., Sommer, L., Arntzen, M.O., Strahm, Y., Morrice, N.A., Divecha, N., and D'Santos, C.S. (2011). Identification of nuclear phosphatidylinosito 4,5-bisphosphate-interacting proteins by neomycin extraction. Mol. Cell. Proteomics 10, 003376.

Liu, X., Gao, Q., Li, P., Zhao, Q., Zhang, J., Li, J., Koseki, H., and Wong, J. (2013). UHRF1 targets DNMT1 for DNA methylation through cooperative binding of hemi-methylated DNA and methylated H3K9. Nat Commun 4, 1563.

Lu, C., and Thompson, C.B. (2012). Metabolic regulation of epigenetics. Cell Metab. 16, 9-17.

Ma, H., Chen, H., Guo, X., Wang, Z., Sowa, M.E., Zheng, L., Hu, S., Zeng, P., Guo, R., Diao, J., et al. (2012). M phase phosphorylation of the epigenetic regulator UHRF1 regulates its physical association with the deubiquitylase USP7 and stability. Proc. Natl. Acad. Sci. USA 109, 4828-4833.

Manzoli, F.A., Cocco, L., Facchini, A., Casali, A.M., Maraldi, N.M., and Grossi, C.E. (1976). Phospholipids bound to acidic nuclear proteins in human $B$ and $T$ lymphocytes. Mol. Cell. Biochem. 12, 67-71.

Maraldi, N.M., Capitani, S., Caramelli, E., Cocco, L., Barnabei, O., and Manzoli, F.A. (1984). Conformational changes of nuclear chromatin related to phospholipid induced modifications of the template availability. Adv. Enzyme Regul. 22, 447-464.

Nady, N., Lemak, A., Walker, J.R., Avvakumov, G.V., Kareta, M.S., Achour, M., Xue, S., Duan, S., Allali-Hassani, A., Zuo, X., et al. (2011). Recognition of multivalent histone states associated with heterochromatin by UHRF1 protein. J. Biol. Chem. 286, 24300-24311.

Ndamukong, I., Jones, D.R., Lapko, H., Divecha, N., and Avramova, Z. (2010). Phosphatidylinositol 5-phosphate links dehydration stress to the activity of ARABIDOPSIS TRITHORAX-LIKE factor ATX1. PLOS ONE 5, e13396.

Nishiyama, A., Yamaguchi, L., Sharif, J., Johmura, Y., Kawamura, T., Nakanishi, K., Shimamura, S., Arita, K., Kodama, T., Ishikawa, F., et al. (2013). Uhrf1-dependent H3K23 ubiquitylation couples maintenance DNA methylation and replication. Nature 502, 249-253. 


\section{Molecular Cell}

Olsen, J.V., Vermeulen, M., Santamaria, A., Kumar, C., Miller, M.L., Jensen, L.J., Gnad, F., Cox, J., Jensen, T.S., Nigg, E.A., et al. (2010). Quantitative phosphoproteomics reveals widespread full phosphorylation site occupancy during mitosis. Sci. Signal. 3, ra3.

Papait, R., Pistore, C., Negri, D., Pecoraro, D., Cantarini, L., and Bonapace, I.M. (2007). Np95 is implicated in pericentromeric heterochromatin replication and in major satellite silencing. Mol. Biol. Cell 18, 1098-1106.

Pasquali, C., Bertschy-Meier, D., Chabert, C., Curchod, M.L., Arod, C., Booth, R., Mechtler, K., Vilbois, F., Xenarios, I., Ferguson, C.G., et al. (2007). A chemical proteomics approach to phosphatidylinositol 3-kinase signaling in macrophages. Mol. Cell. Proteomics 6, 1829-1841.

Patel, D.J., and Wang, Z. (2013). Readout of epigenetic modifications. Annu. Rev. Biochem. 82, 81-118.

Rajakumara, E., Wang, Z., Ma, H., Hu, L., Chen, H., Lin, Y., Guo, R., Wu, F., Li, H., Lan, F., et al. (2011). PHD finger recognition of unmodified histone H3R2 links UHRF1 to regulation of euchromatic gene expression. Mol. Cell 43, 275-284.

Rigbolt, K.T., Prokhorova, T.A., Akimov, V., Henningsen, J., Johansen, P.T., Kratchmarova, I., Kassem, M., Mann, M., Olsen, J.V., and Blagoev, B. (2011). System-wide temporal characterization of the proteome and phosphoproteome of human embryonic stem cell differentiation. Sci. Signal. 4, rs3.

Rose, H.G., and Frenster, J.H. (1965). Composition and metabolism of lipids within repressed and active chromatin of interphase lymphocytes. Biochim. Biophys. Acta 106, 577-591.

Rothbart, S.B., Dickson, B.M., Ong, M.S., Krajewski, K., Houliston, S., Kireev, D.B., Arrowsmith, C.H., and Strahl, B.D. (2013). Multivalent histone engagement by the linked tandem Tudor and PHD domains of UHRF1 is required for the epigenetic inheritance of DNA methylation. Genes Dev. 27, 1288-1298.

Rottach, A., Frauer, C., Pichler, G., Bonapace, I.M., Spada, F., and Leonhardt, H. (2010). The multi-domain protein Np95 connects DNA methylation and histone modification. Nucleic Acids Res. 38, 1796-1804.
Rusten, T.E., and Stenmark, H. (2006). Analyzing phosphoinositides and their interacting proteins. Nat. Methods 3, 251-258.

Schuck, P., and Rossmanith, P. (2000). Determination of the sedimentation coefficient distribution by least-squares boundary modeling. Biopolymers 54, 328-341.

Shah, Z.H., Jones, D.R., Sommer, L., Foulger, R., Bultsma, Y., D’Santos, C., and Divecha, N. (2013). Nuclear phosphoinositides and their impact on nuclear functions. FEBS J. 280, 6295-6310.

Sharif, J., and Koseki, H. (2011). Recruitment of Dnmt1 roles of the SRA protein Np95 (Uhrf1) and other factors. Prog. Mol. Biol. Transl. Sci. 101, 289-310.

Sharif, J., Muto, M., Takebayashi, S., Suetake, I., Iwamatsu, A., Endo, T.A., Shinga, J., Mizutani-Koseki, Y., Toyoda, T., Okamura, K., et al. (2007). The SRA protein Np95 mediates epigenetic inheritance by recruiting Dnmt1 to methylated DNA. Nature 450, 908-912.

Simonsen, A., Lippé, R., Christoforidis, S., Gaullier, J.M., Brech, A., Callaghan, J., Toh, B.H., Murphy, C., Zerial, M., and Stenmark, H. (1998). EEA1 links PI(3)K function to Rab5 regulation of endosome fusion. Nature 394, 494-498.

Wang, C., Shen, J., Yang, Z., Chen, P., Zhao, B., Hu, W., Lan, W., Tong, X., Wu, H., Li, G., and Cao, C. (2011). Structural basis for site-specific reading of unmodified R2 of histone H3 tail by UHRF1 PHD finger. Cell Res. 21, 1379-1382.

Wang, F., Yang, Y.Z., Shi, C.Z., Zhang, P., Moyer, M.P., Zhang, H.Z., Zou, Y., and Qin, H.L. (2012). UHRF1 promotes cell growth and metastasis through repression of p16(ink ${ }^{4}$ a) in colorectal cancer. Ann. Surg. Oncol. 19, 2753-2762.

Xie, S., Jakoncic, J., and Qian, C. (2012). UHRF1 double tudor domain and the adjacent $\mathrm{PHD}$ finger act together to recognize K9me3-containing histone $\mathrm{H} 3$ tail. J. Mol. Biol. 415, 318-328. 